\title{
Catalytic [3+2] Annulation of Aminocyclopropanes for the Enantiospecific Synthesis of Cyclopentylamines. **
}

\author{
Florian de Nanteuil and Jérôme Waser*
}

Cyclic structures are encountered in the core of most bioactive synthetic and natural products. Carbo- and heterocyclic rings give rigidity to molecules, allowing the fixation of functional groups in the space. As a result, specific interactions with biomolecules without important entropy costs become possible. Nitrogen-based functional groups are especially important in synthetic and medicinal chemistry. In this respect, cyclopentylamines constitute an important subclass of cyclic structures, present in both relatively simple natural products, such as the antibiotic and antiviral aristeromycin (1), ${ }^{[1]}$ or in dauntingly complex polycyclic structures, such as palau'amine (2), one of the most sought for targets in modern organic chemistry (Figure 1). ${ }^{[2]}$ They are also part of purely synthetic drugs. For example, ramipril (3), an angiotensinconverting enzyme (ACE) inhibitor, was one of the top 100 generic drugs in 2008 for the treatment of high blood pressure and heart failure. $^{[3]}$ Consequently, the development of new synthetic procedures to access polysubstituted cyclopentyl amines is an important task for organic chemists. ${ }^{[4]}$

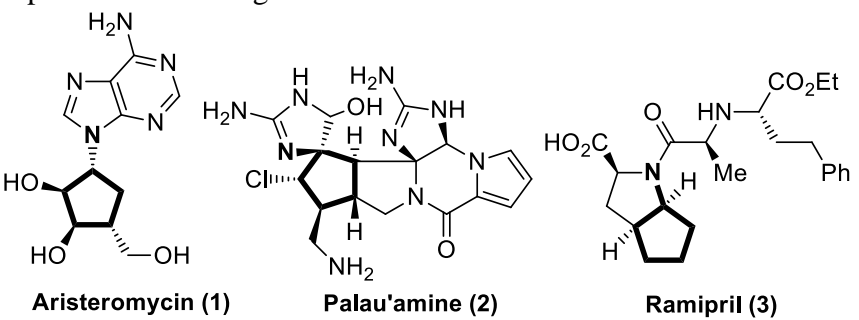

Figure 1. Cyclopentyl Amines in Natural and Synthetic Products.

One of the most efficient ways to access 5-membered rings is by concerted cycloaddition reactions or related stepwise annulation processes. In particular, the formal $[3+2]$ cycloaddition, more correctly called [3+2] annulation, of donor-acceptor activated cyclopropanes ${ }^{[5]}$ with alkenes or alkynes, ${ }^{[6]}$ carbonyls $^{[7]}$ and imines ${ }^{[8]}$ has been highly successful in the last decades for the synthesis of cyclopentanes/enes, tetrahydrofurans and pyrrolidines respectively. Cyclopropanes bearing electron-donating oxygen or aromatic

[*] F. de Nanteuil and Prof. Dr. J. Waser Laboratory of Catalysis and Organic Synthesis Ecole Polytechnique Fédérale de Lausanne EPFL SB ISIC LCSO, BCH 4306, 1015 Lausanne $(\mathrm{CH})$ Fax: (+)41216939700 E-mail: jerome.waser@epfl.ch Homepage: http://lcso.epfl.ch/

[**] EPFL, F. Hoffmann-La Roche Ltd, and SNF (grant number 200021_129874) are acknowledged for financial support. Dr. Rosario Scopelliti (EPFL) is acknowledged for the X-ray studies.

Supporting information for this article is available on the WWW under http://www.angewandte.org or from the author. substituents have been especially useful in Lewis or Brønsted acidcatalyzed reactions. In contrast, annulation reactions of aminocyclopropanes have been limited to radical-initiated ring opening involving electron-rich amines. ${ }^{[9]}$ To the best of our knowledge, no catalytic [3+2] annulation of aminocyclopropanes has ever been reported. Donor-acceptor aminocyclopropanes have been mostly used as 1,4-imino carbonyl precursors, ${ }^{[10]}$ as well as in a few rare ring-opening reactions. ${ }^{[11]}$ In 2010 , we applied them in an efficient formal homo-Nazarov cyclization for the synthesis of natural alkaloids. ${ }^{[12]}$ However, a formal cycloaddition approach would be inherently more convergent and efficient to access molecular complexity. Herein, we would like to report phthalimidesubstituted acceptor cyclopropanes as unique partners in [3+2] annulation reactions with silyl and alkyl enol ethers (Scheme 1). High yields and diastereo-selectivities were obtained using $\mathrm{SnCl}_{4}$ as catalyst with a broad range of enol ethers as substrates. The reaction was stereospecific in relation to the configuration of the enol ether and enantiospecific, giving an asymmetric access to chiral cyclopentylamines. Finally, the use of $\operatorname{In}(\mathrm{OTf})_{3}$ as catalyst gave access to important acyclic $\beta$-amino ketones and deprotection of the phthalimide group, as well as further functionalization, was possible under mild conditions.

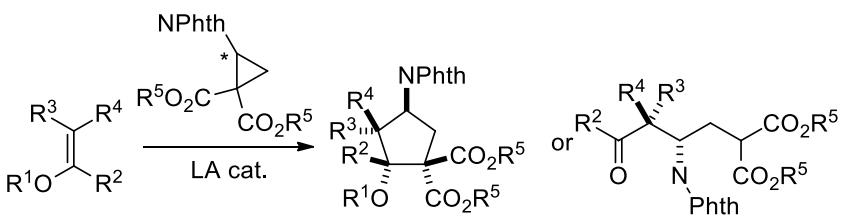

Scheme 1. Lewis Acid (LA)-catalyzed reactions of aminocyclopropanes with enol ethers. PhthN = Phthalimide

As a model system to study the annulation reaction with several aminocyclopropanes 4a-f, stable enol silyl 5a was chosen as an electron-rich alkene, as it was expected to have a higher reactivity than non-activated alkenes and should also allow a good control over the regioselectivity (Table 1). We started with $\mathrm{SnCl}_{4}$ as Lewis acid in dichloromethane, as these conditions had been successful in the case of oxygen-substituted cyclopropanes. ${ }^{[6 f]}$ However, no reaction was observed with cyclopropanes $4 \mathbf{a}, \mathbf{4 b}$ and $\mathbf{4 c}$ bearing a $\mathrm{Cbz}$ protected amine, a lactam or an oxazolidinone substituent respectively (entries 1-3). At this point, we attempted to enhance the reactivity of lactam- and oxazolidinone-substituted cyclopropanes by the introduction of a second ester group. ${ }^{[6 \mathrm{~d}, 6 \mathrm{~h}, 7 \mathrm{c}-\mathrm{e}]}$ However, the obtained cyclopropanes were unstable and already decomposed during purification. These results further emphasized how difficult it is to modulate the reactivity of aminocyclopropanes when compared with other donor-acceptor cyclopropanes. In order to decrease the electron-donor ability of the nitrogen group, phthalimidecyclopropane 4d was investigated next (entry 4). For the first time, a clean annulation was observed, giving exclusively the trans diastereoisomer 6da of the desired cyclopentylamine! ${ }^{[13]}$ Importantly, phthalimide-substituted cyclopropanes can be obtained 
in a single step on a multi-gram scale from commercially available $N$-vinylphthalimide and diazomalonates by Rh-catalyzed cyclopropanation. ${ }^{[14]}$ On the other hand, removal of one of the ester groups led again to an unreactive cyclopropane (4e, entry 5 ). The use of $\mathrm{SnCl}_{4}$ at room temperature led to partial ring opening (entry 6). At this point, several other Lewis acids successful in related $[3+2]$ annulations ${ }^{[6-8]}$ were examined (entries 7-12). Immediately, the exceptional reactivity of cyclopropane $\mathbf{4 d}$ became apparent, as full conversion could be obtained with a broad range of Lewis acids. ${ }^{[15]}$ Nevertheless, a mixture of cyclization and homo-aldol products was obtained in most cases, with the exception of In(OTf $)_{3}$ (entry 11) and $\mathrm{HNTf}_{2}$ (entry 12), which led to complete ring opening. Finally, full conversion to a single diastereoisomer was also obtained with dimethyl ester $\mathbf{4 f}$ (entry 13) and the cyclopentylamine could be obtained in $95 \%$ isolated yield after column chromatography in this case.

Table 1. Optimization of the [3+2] Annulation.
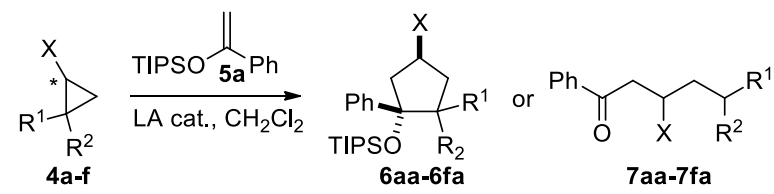

\begin{tabular}{|c|c|c|c|c|c|}
\hline entry & $\mathrm{R}^{1} / \mathrm{R}^{2}$ & $X$ & $\begin{array}{c}\text { Catalyst, } \\
\text { Conditions }^{[a]}\end{array}$ & Conversion $^{[b]}$ & $\begin{array}{l}\text { ratio } \\
6: 7^{[c]}\end{array}$ \\
\hline 1 & $\mathrm{CO}_{2} \mathrm{Et} / \mathrm{H}$ & & $\mathrm{SnCl}_{4},-78^{\circ} \mathrm{C}$ & $0 \%$ & - \\
\hline 2 & $\mathrm{CO}_{2} \mathrm{Et} / \mathrm{H}$ & & $\mathrm{SnCl}_{4},-78^{\circ} \mathrm{C}$ & $0 \%$ & - \\
\hline 3 & $\mathrm{CO}_{2} \mathrm{Et} / \mathrm{H}$ & & $\mathrm{SnCl}_{4},-78^{\circ} \mathrm{C}$ & $0 \%$ & - \\
\hline
\end{tabular}

$4 \mathrm{CO}_{2} \mathrm{Et} / \mathrm{CO}_{2} \mathrm{Et} \quad$ NPhth, (4d) $\mathrm{SnCl}_{4},-78{ }^{\circ} \mathrm{C} 100 \%(98 \%)^{[\mathrm{d}]}>20: 1$

$5 \quad \mathrm{CO}_{2} \mathrm{Et} / \mathrm{H} \quad \mathrm{NPhth},(\mathbf{4 e}) \mathrm{SnCl}_{4},-78{ }^{\circ} \mathrm{C} \quad 0 \% \quad$ -

$6 \quad \mathrm{CO}_{2} \mathrm{Et} / \mathrm{CO}_{2} \mathrm{Et} \quad$ NPhth, (4d) $\quad \mathrm{SnCl}_{4}, \mathrm{rt} \quad 100 \% \quad 1: 1$

$7 \quad \mathrm{CO}_{2} \mathrm{Et} / \mathrm{CO}_{2} \mathrm{Et} \quad$ NPhth, (4d) $\mathrm{Cu}(\mathrm{OTf})_{2}$, rt $\quad 100 \% \quad 5: 1$

$8 \quad \mathrm{CO}_{2} \mathrm{Et} / \mathrm{CO}_{2} \mathrm{Et} \quad$ NPhth, (4d) $\mathrm{Yb}(\mathrm{OTf})_{2}$, rt $\quad 100 \% \quad 5: 1$

$9 \mathrm{CO}_{2} \mathrm{Et} / \mathrm{CO}_{2} \mathrm{Et} \quad$ NPhth, (4d) $\mathrm{Sn}(\mathrm{OTf})_{2}$, rt $\quad 100 \% \quad 2: 1$

$10 \mathrm{CO}_{2} \mathrm{Et} / \mathrm{CO}_{2} \mathrm{Et}$ NPhth, (4d) $\mathrm{Sc}(\mathrm{OTf})_{3}$, rt $\quad 100 \% \quad 1: 3$

$11 \mathrm{CO}_{2} \mathrm{Et} / \mathrm{CO}_{2} \mathrm{Et} \quad$ NPhth, (4d) In(OTf) $)_{3}$, rt $\quad 100 \% \quad<1: 20$

$12 \mathrm{CO}_{2} \mathrm{Et} / \mathrm{CO}_{2} \mathrm{Et}$ NPhth, (4d) $\mathrm{HNTf}_{2}$, rt $\quad 100 \% \quad<1: 20$

$13 \mathrm{CO}_{2} \mathrm{Me} / \mathrm{CO}_{2} \mathrm{Me}$ NPhth, (4f) $\mathrm{SnCl}_{4},-78{ }^{\circ} \mathrm{C} 100 \%(95 \%)^{[\mathrm{d}]}>20: 1$

[a] Reaction conditions: $0.040 \mathrm{mmol}$ 4, $0.060 \mathrm{mmol} 5 \mathrm{a}, 0.008 \mathrm{mmol}$ catalyst, $0.5 \mathrm{~mL} \mathrm{CH} \mathrm{Cl}_{2}, 1 \mathrm{~h}$. [b] Conversion of 4 determined by ${ }^{1} \mathrm{H}-$ NMR. [c] Determined by ${ }^{1} \mathrm{H}-\mathrm{NMR}$ in the crude mixture. [d] Isolated yield after column chromatography on $0.30 \mathrm{mmol}$ scale.

When considering the importance of cyclopentylamines, and the lack of general methods for their synthesis, we decided to investigate first the scope of the cycloaddition reaction using $\mathrm{SnCl}_{4}$ as catalyst with cyclopropane $\mathbf{4 f}$ (Table 2 ). ${ }^{[16]}$ The size of the silyl group on the enol ether had no influence on yield or selectivity and the desired product was obtained in quantitative yield with very high diastereoselectivity (entries 1-3). ${ }^{[17]}$ Both electron-donating and electron-withdrawing groups in para or ortho position were well tolerated on enol ethers derived from acetophenone (entries 4-9).
Table 2. Scope of the [3+2] Annulation.

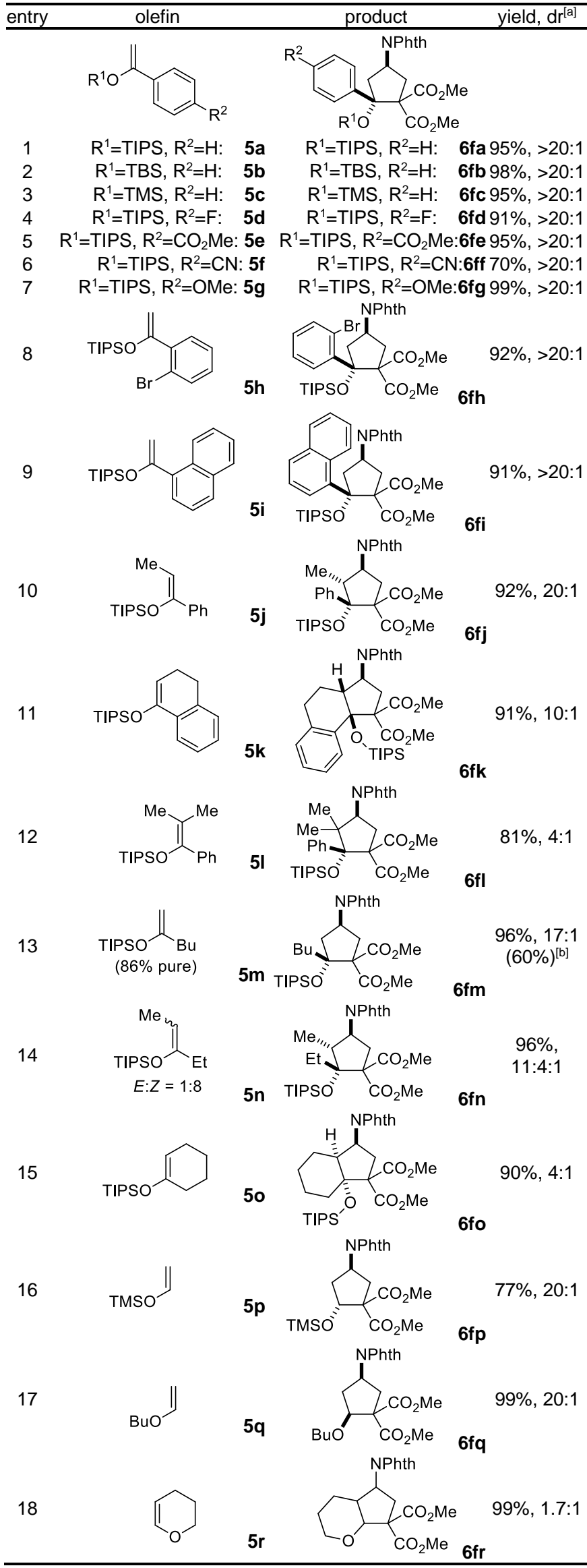

[a] Reaction conditions: $0.45 \mathrm{mmol} 5,0.30 \mathrm{mmol} \mathbf{4 f}, 0.015 \mathrm{mmol}$ $\mathrm{SnCl}_{4}$, in $2 \mathrm{~mL} \mathrm{CH}_{2} \mathrm{Cl}_{2}, 1 \mathrm{~h},-78^{\circ} \mathrm{C}$. [b] Yield of major product determined by ${ }^{1} \mathrm{H}$ NMR. Contaminated by other regioisomers. 
The yields were quantitative, except in the case of a cyanophenyl-substituted enol ether $\mathbf{5 f}$, which led to a slow reaction with incomplete conversion (entry 6). The reaction was stereospecific with $Z$ enol ether $\mathbf{5 j}$ giving a highly substituted cyclopentylamine $\mathbf{6 f j}$ with perfect diastereoselectivity (entry 10). Interestingly, the opposite diastereoisomer with the nitrogen and the oxygen in a syn relationship was obtained using the enol ether $\mathbf{5 k}$ derived from tetralone (entry 11). Trisubstituted enol ether $\mathbf{5} \mathbf{l}$ could also be used, giving a sterically congested product with two allcarbon $\mathrm{N}$-substituted quaternary centers and one tertiary silyloxy group next to each other (entry 12). We then turned to silyl enol ethers derived from aliphatic ketones (entries 13-15). Gratifyingly, these substrates also worked very well in the annulation reaction, but the difficulties in obtaining distereomerically pure regioisomers of the enol ethers led to product mixtures in case of acyclic substrates (entry 13-14). The diastereoselectivity was moderate only in the case of cyclic enol ether 50 (entry 15). The commercially available silyl enol ether 5p derived from acetaldehyde gave the secondary alcohol product also with high diastereoselectivity, demonstrating that the reaction was not limited to enol ethers derived from ketones (entry 16). Finally, alkyl enol ethers could also be used (entries 17-18). Good diastereoselectivity was obtained in the case of acyclic substrate $\mathbf{5 q}$ (entry 17), but cyclic substrate $\mathbf{5 r}$ gave no selectivity in the annulation reaction (entry 18). Taken together, these results emphasize the exceptional properties of cyclopropane $\mathbf{4 f}$ in [3+2] annulation reactions with enol ethers. Most reported methods with other classes of donor-acceptor cyclopropanes for annulation with alkenes led to lower yields and diastereoselectivities or a limited scope. ${ }^{[6]}$

When considering previous works on annulation reactions of donor-acceptor cyclopropanes, ${ }^{[7 c]}$ two mechanisms could be envisaged for the first step of the annulation: either as stepwise process involving ring-opening of the cyclopropane to form a zwitterionic intermediate followed by attack of the enol ether, or a process via an "intimate ion pair "[7d] involving a concerted attack of the enol ether anti to the malonates. ${ }^{[18]}$ When using an enantiopure aminocyclopropane, racemization would be expected in the former case, whereas the latter would lead to an enantiospecific reaction. In the event, when enantiopure aminocyclopropane $\mathbf{4 f}$ was used, ${ }^{[19]}$ an enantiospecific reaction was observed with all substrates except in the formation of $\mathbf{6 f h}$ (Scheme 2), giving access to enantiopure cyclopentylamines.

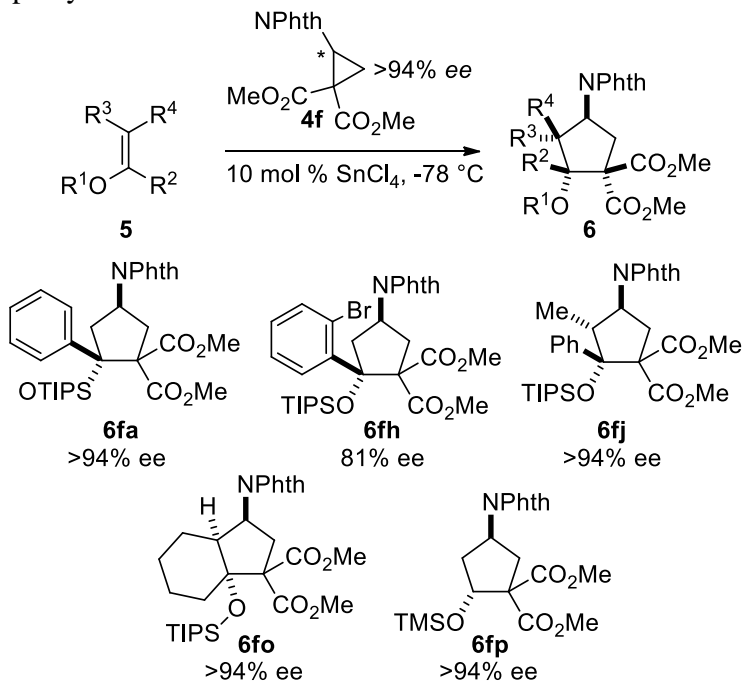

Scheme 2. Enantiospecific reaction of cyclopropane $\mathbf{4 f}$.
To further establish the synthetic potential of the method, a few transformations of the cyclopentylamine products were examined (Scheme 3). Ring-opened product $\mathbf{7 f a}$ can be obtained in one single step from aminocyclopropane $\mathbf{4 f}$ using $\operatorname{In}(\mathrm{OTf})_{3}$ as catalyst, but in this case, a partial racemization was observed (Scheme 3, A). In contrast, reaction of $\mathbf{6} \mathbf{f a}$ in the presence of $\operatorname{In}(\mathrm{OTf})_{3}$ led to a smooth transformation to the acyclic $\beta$-amino ketone 7 fa without loss of the enantiopurity. Also $\alpha$-substituted $\beta$-amino ketone $\mathbf{7 f j}$ can be obtained directly from $4 \mathbf{f}$, but with only $4: 1 \mathrm{dr}$ (Scheme $3, \mathbf{B}$ ). If cyclopentylamine $\mathbf{6} \mathbf{f j}$ is formed prior to ring-opening, perfect syn diastereoselectivity is observed. As stereocontrol in the case of acyclic products is especially challenging, this is an important result. Finally, it is also possible to keep the cyclopentane ring in further transformations (Scheme 3, C). Deprotection of the phthalimide was possible under mild conditions. ${ }^{[20]}$ Two isomeric cyclopentenylamines $\mathbf{9}$ and $\mathbf{1 0}$ could be obtained using either trimethylsilyl triflate or Krapcho decarboxylation conditions, ${ }^{[21]}$ respectively. The obtained cyclopentenylamines are themselves parts of important bioactive compounds, such as the drug Abacavir, used in the treatment of HIV infection, ${ }^{[22]}$ or can be used as platforms for further functionalization.

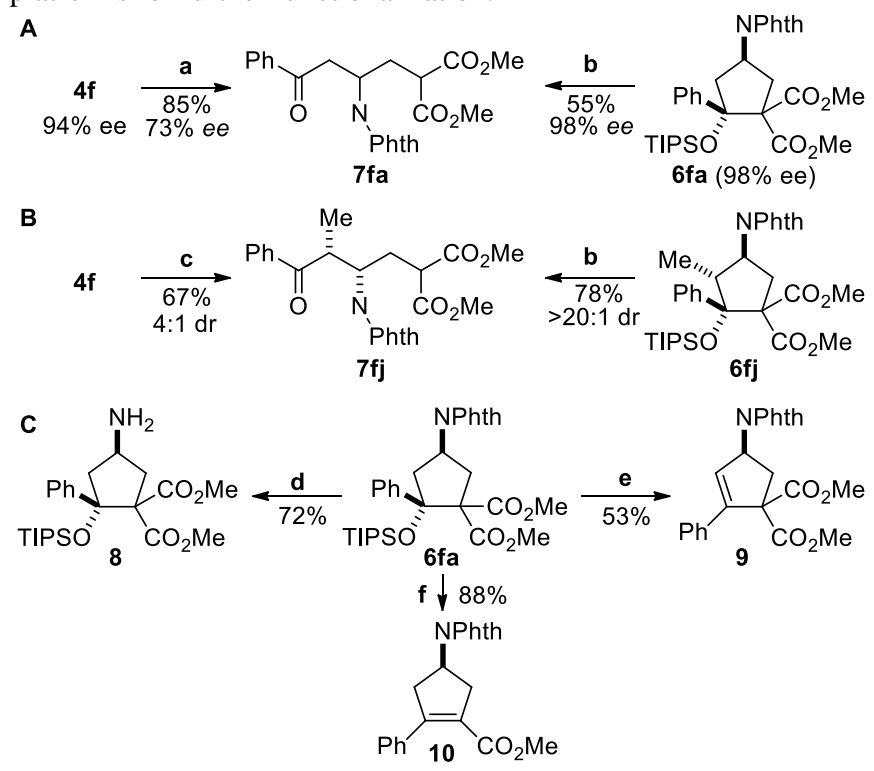

Scheme 3. Transformations of the cyclopentyl amines. Reaction conditions: a) $5 \mathrm{a}, 20 \mathrm{~mol} \% \operatorname{In}(\mathrm{OTf})_{3}, \mathrm{CH}_{2} \mathrm{Cl}_{2}, \mathrm{RT}$; b) $20 \mathrm{~mol} \%$ $\ln (\mathrm{OTf})_{3}, \mathrm{CH}_{2} \mathrm{Cl}_{2}, \mathrm{RT}$; c) 5j, $20 \mathrm{~mol} \% \ln (\mathrm{OTf})_{3}, \mathrm{CH}_{2} \mathrm{Cl}_{2}, \mathrm{RT}$; d) 1,2diamino-ethane, $\mathrm{PrOH}, 80^{\circ} \mathrm{C}$; e) TMSOTf, $\mathrm{CH}_{2} \mathrm{Cl}_{2}, 0^{\circ} \mathrm{C}$; f) $\mathrm{NaCl}$, DMSO, $\mathrm{H}_{2} \mathrm{O}, 170^{\circ} \mathrm{C}$.

In summary, we have reported the first catalytic [3+2] annulation of aminocyclopropanes with enol ethers. The introduction of a phthalimide group on a cyclopropane diester was key to enable high yield and selectivity in the reaction. Quantitative yields and a very broad scope made the reaction highly useful for the synthesis of substituted cyclopentylamines, for which truly general synthetic methods are scarce. As the reaction is enantiospecific, enantiopure cyclopentylamines can be easily obtained. Finally, substituted $\beta$-amino ketones or useful cyclopentenylamines can easily be obtained, either directly from the cyclopropane or from the cyclopentylamine products.

Received: ((will be filled in by the editorial staff))

Published online on ((will be filled in by the editorial staff)) 
Keywords: Aminocyclopropanes - CATALYSIS - Cycloaddition react. - Cyclopentylamines · Enantiospecific Reaction - Stereoselectivity.

[1] a) T. Kusaka, H. Yamamoto, M. Shibata, M. Muroi, T. Kishi, K. Mizuno, J. Antibiot. 1968, 21, 255; b) B. M. Trost, G. H. Kuo, T. Benneche, J. Am. Chem. Soc. 1988, 110, 621.

[2] a) M. Kock, A. Grube, I. B. Seiple, P. S. Baran, Angew. Chem. 2007 119, 6706; Angew. Chem., Int. Ed. 2007, 46, 6586; b) I. B. Seiple, S. $\mathrm{Su}$, I. S. Young, C. A. Lewis, J. Yamaguchi, P. S. Baran, Angew. Chem. 2010, 122, 114; Angew. Chem., Int. Ed. 2010, 49, 1095.

[3] S. Yusuf, K. K. Teo, J. Pogue, L. Dyal, I. Copland, H. Schumacher, B. Ingelheim, G. Dagenais, P. Sleight, C. Anderson N. Engl. J. Med. 2008, 358, 1547.

[4] For a review on the synthesis of cyclopentane-derived natural products, see: a) B. Heasley, Eur. J. Org. Chem. 2009, 1477; For some recent examples for the synthesis of cyclopentylamines, see: b) B. Tan Z. G. Shi, P. J. Chua, G. F. Zhong, Org. Lett. 2008, 10, 3425; c) B. Tan, P. J. Chua, X. F. Zeng, M. Lu, G. F. Zhong, Org. Lett. 2008, 10, 3489; d) D. N. Tran, N. Cramer, Angew. Chem. 2010, 122, 8357; Angew. Chem., Int. Ed. 2010, 49, 8181. e) G. R. Boyce, J. S. Johnson, Angew. Chem., Int. Ed. 2010, 49, 8930.

[5] For reviews on activated cyclopropanes, especially donor-acceptor cyclopropanes, see: a) H. U. Reissig, R. Zimmer, Chem. Rev. 2003, 103, 1151; b) F. Gnad, O. Reiser, Chem. Rev. 2003, 103, 1603; c) M Yu, B. L. Pagenkopf, Tetrahedron 2005, 61, 321; d) D. Agrawal, V. K Yadav, Chem. Commun. 2008, 6471; e) C. A. Carson, M. A. Kerr, Chem. Soc. Rev. 2009, 38, 3051; f) F. De Simone, J. Waser, Synthesis 2009, 3353; g) T. P. Lebold, M. A. Kerr, Pure Appl. Chem. 2010, 82, 1797

[6] a) J. E. Dolfini, K. Menich, P. Corliss, Tetrahedron Lett. 1966, 4421; b) E. J. Corey, R. D. Balanson, Tetrahedron Lett. 1973, 3153; c) J. P. Marino, E. Laborde, J. Am. Chem. Soc. 1985, 107, 734; d) R. B. Beal, M. A. Dombroski, B. B. Snider, J. Org. Chem. 1986, 51, 4391; e) K. Saigo, S. Shimada, T. Shibasaki, M. Hasegawa, Chem. Lett. 1990, 1093; f) M. Komatsu, I. Suehiro, Y. Horiguchi, I. Kuwajima, Synlett 1991, 771; g) Y. Horiguchi, I. Suehiro, A. Sasaki, I. Kuwajima, Tetrahedron Lett. 1993, 34, 6077; h) M. A. Kerr, R. G. Keddy, Tetrahedron Lett. 1999, 40, 5671; i) D. B. England, T. D. O. Kuss, R. G. Keddy, M. A. Kerr, J. Org. Chem. 2001, 66, 4704; j) V. K. Yadav, V. Sriramurthy, Org. Lett. 2004, 6, 4495; k) V. K. Yadav, V. Sriramurthy, Angew. Chem. 2004, 116, 2723; Angew. Chem., Int. Ed. 2004, 43, 2669; 1) K. Takasu, S. Nagao, M. Ihara, Adv. Synth. Catal. 2006, 348, 2376; m) C. Venkatesh, P. P. Singh, H. Ila, H. Junjappa, Eur. J. Org. Chem. 2006, 5378; n) L. Liu, J. Montgomery, J. Am. Chem. Soc. 2006, 128, 5348; o) B. Bajtos, M. Yu, H. D. Zhao, B. L. Pagenkopf, J. Am. Chem. Soc. 2007, 129, 9631; p) J. Fang, J. Ren, Z. W. Wang, Tetrahedron Lett. 2008, 49, 6659; q) X. B. Qi, J. M. Ready, Angew. Chem. 2008, 120, 7176; Angew. Chem., Int. Ed. 2008, 47, 7068; r) A. O. Chagarovskiy, E. M. Budynina, O. A. Ivanova, Y. K. Grishin, I. V. Trushkov, P. V. Verteletskii, Tetrahedron 2009, 65, 5385; s) W. Li, M. Shi, J. Org. Chem. 2009, 74, 856; t) J. P. Qu, C. Deng, J. Zhou, X. L. Sun, Y. Tang, J. Org. Chem. 2009, 74, 7684; u) Z. Lu, M. H. Shen, T. P. Yoon, J. Am. Chem. Soc. 2011, 133, 1162.

[7] For a few selected examples, see: a) S. Shimada, Y. Hashimoto, A. Sudo, M. Hasegawa, K. Saigo, J. Org. Chem. 1992, 57, 7126; b)Y. Sugita, K. Kawai, I. Yokoe, Heterocycles 2001, 55, 135; c) P. D. Pohlhaus, J. S. Johnson, J. Am. Chem. Soc. 2005, 127, 16014; d) P. D. Pohlhaus, S. D. Sanders, A. T. Parsons, W. Li, J. S. Johnson, J. Am. Chem. Soc. 2008, 130, 8642; e) A. T. Parsons, J. S. Johnson, J. Am. Chem. Soc. 2009, 131, 3122; f) A. Karadeolian, M. A. Kerr, Angew. Chem. 2010, 122, 1151; Angew. Chem., Int. Ed. 2010, 49, 1133; g) S. Y. Xing, W. Y. Pan, C. Liu, J. Ren, Z. W. Wang, Angew. Chem. 2010, 122, 3283; Angew. Chem., Int. Ed. 2010, 49, 3215.

[8] For a few selected examples, see: a) P. B. Alper, C. Meyers, A. Lerchner, D. R. Siegel, E. M. Carreira, Angew. Chem. 1999, 111, 3379; Angew. Chem., Int. Ed. 1999, 38, 3186; b) M. Lautens, W. S. Han, J. Am. Chem. Soc. 2002, 124, 6312; c) M. Yu, B. L. Pagenkopf, J. Am. Chem. Soc. 2003, 125, 8122; d) C. A. Carson, M. A. Kerr, J. Org. Chem. 2005, 70, 8242; e) S. K. Jackson, A. Karadeolian, A. B. Driega, M. A. Kerr, J. Am. Chem. Soc. 2008, 130, 4196; f) A. T.
Parsons, A. G. Smith, A. J. Neel, J. S. Johnson, J. Am. Chem. Soc. 2010, $132,9688$.

[9] a) J. D. Ha, J. W. Lee, S. C. Blackstock, J. K. Cha, J. Org. Chem 1998, 63, 8510; b) C. Madelaine, Y. Six, O. Buriez, Angew. Chem. 2007, 119, 8192; Angew. Chem., Int. Ed. 2007, 46, 8046.

[10] a) E. Wenkert, T. Hudlicky, H. D. H. Showalter, J. Am. Chem. Soc. 1978, 100, 4893; b) E. Wenkert, T. Hudlicky, J. Org. Chem. 1988, 53, 1953

[11] For a few selected examples, see: a) M. Ikeda, S. Matsugashita, Y. Tamura, J. Chem. Soc. Perkin Trans. 1 1977, 1770; b) T. Inokuchi, S Takagishi, F. Akahoshi, S. Torii, Chem. Lett. 1987, 1553; c) H. M. L. Davies, J. J. Matasi, L. M. Hodges, N. J. S. Huby, C. Thornley, N. Kong, J. H. Houser, J. Org. Chem. 1997, 62, 1095; d) J. W. Lee, J. S. U, S. C. Blackstock, J. K. Cha, J. Am. Chem. Soc. 1997, 119, 10241 ; e) J. Barluenga, A. Ballesteros, J. Santamaria, R. B. de la Rua, E Rubio, M. Tomas, J. Am. Chem. Soc. 2000, 122, 12874; f) H. B. Lee, M. J. Sung, S. C. Blackstock, J. K. Cha, J. Am. Chem. Soc. 2001, 123, 11322 ; g) K. Wimalasena, H. B. Wickman, M. P. D. Mahindaratne, Eur. J. Org. Chem. 2001, 3811; h) L. Larquetoux, N. Ouhamou, A Chiaroni, Y. Six, Eur. J. Org. Chem. 2005, 4654; i) D. Sampedro, A. Soldevilla, M. A. Rodriguez, P. J. Campos, M. Olivucci, J. Am. Chem. Soc. 2005, 127, 441; j) S. Mangelinckx, N. De Kimpe, Synlett 2006, $369 ;$ k) C. Tanguy, P. Bertus, J. Szymoniak, O. V. Larionov, A. de Meijere, Synlett 2006, 2339; 1) R. P. Reddy, H. M. L. Davies, J. Am. Chem. Soc. 2007, 129, 10312; m) J. Yang, H. X. Wu, L. Q. Shen, Y. Qin, J. Am. Chem. Soc. 2007, 129, 13794; n) Z. W. Zuo, W. Q. Xie, D. W. Ma, J. Am. Chem. Soc. 2010, 132, 13226; o) D. Zhang, H. Song, Y Qin, Acc. Chem. Res. 2011, 44, 447.

[12] a) F. De Simone, J. Gertsch, J. Waser, Angew. Chem. 2010, 122, 5903; Angew. Chem., Int. Ed. 2010, 49, 5767; b) F. De Simone, J. Waser, Synlett 2011, 589

[13] The structure and relative configuration of $\mathbf{6 d a}$ was determined by Xray analysis. See supporting information.

[14] F. Gonzalez-Bobes, M. D. B. Fenster, S. Kiau, L. Kolla, S. Kolotuchin, M. Soumeillant, Adv. Synth. Catal. 2008, 350, 813.

[15] See supporting information for a full range of tested Lewis and Brønsted acids.

[16] The dimethylcyclopropanedicarboxylate was used preferentially as the products were easier to characterize by NMR methods.

[17] The structures and relative configurations of compounds $6 \mathbf{d a}, 6 \mathbf{6 k}, 6 \mathbf{6 p}$ and $\mathbf{6 f r}$ was confirmed by X-ray analysis, and of compounds $\mathbf{6 f j}$ by 2D-NMR analysis. The structures and relative configurations of the others were deduced by analogy. The phthalimide group was instrumental in giving high crystallinity to most products in this work. See supporting information.

[18] The determination of the absolute configuration of the aminocyclopropane and cyclopentylamine would allow us to confirm that the reaction in fact proceeded via inversion. These further studies on the reaction mechanism are currently ongoing in our group and the result will be reported in a future full account of our work.

[19] The enantiomers of cyclopropanes $\mathbf{4 f}$ were conveniently separated by chiral column chromatography. Investigations towards a resolution or an asymmetric synthesis of $\mathbf{4 f}$ are currently ongoing in our group.

[20] O. Kanie, S. C. Crawley, M. M. Palcic, O. Hindsgaul, Carbohydr. Res 1993, 243, 139. Deprotection of the silyl group without retro-aldol reaction could not be achieved so far.

[21] a) A. P. Krapcho, J. F. Weimaster, J. M. Eldridge, E. G. E. Jahngen Jr., A. J. Lovey, W. P. Stephens, J. Org. Chem., 1978, 43, 138; For an alternative approach towards cyclopentenylamines starting from aminocyclopropanes, see: b) C. M. Williams, A. de Meijere, J. Chem. Soc. Perkin Trans. 1 1998, 3699.

[22] P. S. Hervey, C. M. Perry, Drugs 2000, 60, 447. 


\section{Synthetic Method}

Florian de Nanteuil and Jérôme Waser

Page - Page

Catalytic [3+2] Annulation of Aminocyclopropanes for the Enantiospecific Synthesis of Cyclopentylamines.

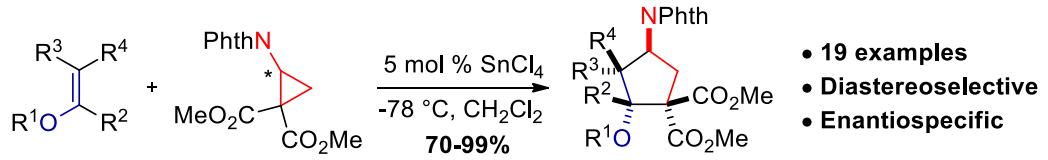

Also with Nitrogen: The first catalytic [3+2] annulation of aminocyclopropanes with enol ethers is reported. The reaction worked with easily accessible phthalimidocyclopropanes using $5 \mathrm{~mol}_{\%} \mathrm{SnCl}_{4}$ in nearly quantitative yields. Polysubstituted cyclopentyl amines, which are often present in bioactive compounds, were obtained with high diastereoselectivity and enantiospecificity. Mild conditions were also developed to access acyclic $\beta$-amino ketones and cyclopentene amines. 
Catalytic Formal [3+2] Cycloaddition of Aminocyclopropanes for the Enantiospecific Synthesis of Cyclopentyl Amines. **

Florian de Nanteuil and Jérôme Waser* 


\section{Contents}

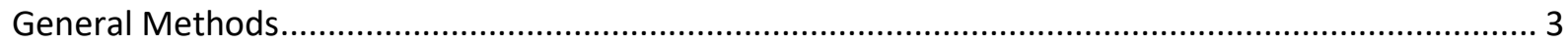

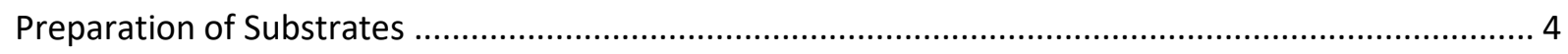

Synthesis of aminocyclopropane

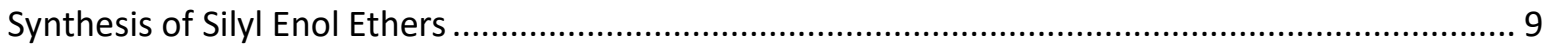

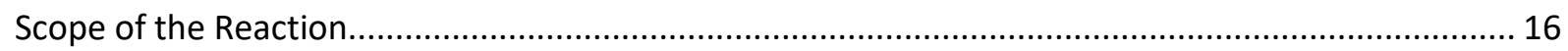

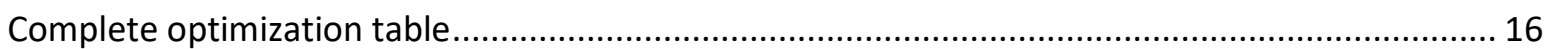

General procedure for the $\mathrm{SnCl}_{4}$-catalyzed synthesis of $\mathrm{N}$-phthalimide aminocyclopentane :........ 17

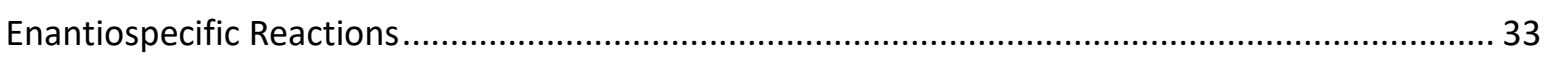

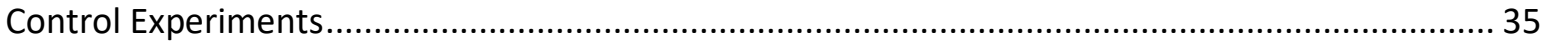

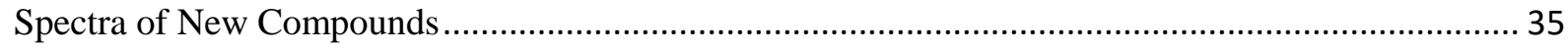




\section{$\underline{\text { General Methods }}$}

All reactions were carried out in oven dried glassware under an atmosphere of nitrogen, unless stated otherwise. For quantitative flash chromatography technical grade solvents were used. For flash chromatography for analysis, HPLC grade solvents from Sigma-Aldrich were used. THF, Et2O, $\mathrm{CH} 3 \mathrm{CN}$, toluene, hexane and $\mathrm{CH} 2 \mathrm{Cl} 2$ were dried by passage over activated alumina under nitrogen atmosphere ( $\mathrm{H} 2 \mathrm{O}$ content $<30 \mathrm{ppm}$, Karl-Fischer titration). NEt3 and pyridine were distilled under nitrogen from KOH. All chemicals were purchased from Acros, Aldrich, Fluka, VWR, Aplichem or Merck and used as such unless stated otherwise. Chromatographic purification was performed as flash chromatography using Macherey-Nagel silica 40-63, $60 \AA$, using the solvents indicated as eluent with 0.1-0.5 bar pressure. TLC was performed on Merck silica gel 60 F254 TLC glass plates or aluminium plates and visualized with UV light, permanganate stain, CAN stain or Anisaldehyde stain. Melting points were measured on a Büchi B-540 melting point apparatus using open glass capillaries, the data is uncorrected. 1H-NMR spectra were recorded on a Brucker DPX-400 $400 \mathrm{MHz}$ spectrometer in chloroform-d, DMSO-d6, CD2Cl2 or CD3OD, all signals are reported in ppm with the internal chloroform signal at $7.26 \mathrm{ppm}$, the internal DMSO signal at $2.50 \mathrm{ppm}$, the internal $\mathrm{CD}_{2} \mathrm{Cl}_{2}$ signal at $5.31 \mathrm{ppm}$, or the internal methanol signal at $3.30 \mathrm{ppm}$ as standard. The data is being reported as $(\mathrm{s}=$ singlet, $\mathrm{d}=$ doublet, $\mathrm{t}=$ triplet, $\mathrm{q}=$ quadruplet, $\mathrm{qi}=$ quintet, $\mathrm{m}=$ multiplet or unresolved, $\mathrm{br}=$ broad signal, coupling constant(s) in $\mathrm{Hz}$, integration; interpretation). 13C-NMR spectra were recorded with 1H-decoupling on a Brucker DPX-400 100 MHz spectrometer in chloroform-d, DMSO-d6, CD2Cl2 or CD3OD, all signals are reported in ppm with the internal chloroform signal at $77.0 \mathrm{ppm}$, the internal DMSO signal at $39.5 \mathrm{ppm}$, the internal $\mathrm{CD} 2 \mathrm{Cl} 2$ signal at $53.5 \mathrm{ppm}$ or the internal methanol signal at $49.0 \mathrm{ppm}$ as standard. Infrared spectra were recorded on a JASCO FT-IR B4100 spectrophotometer with an ATR PRO410-S and a ZnSe prisma and are reported as $\mathrm{cm}-1$ ( $\mathrm{w}=$ weak, $\mathrm{m}=$ medium, $\mathrm{s}=$ strong, sh $=$ shoulder). Gas chromatographic and low resolution mass spectrometric measurements were performed on a Perkin-Elmer Clarus 600 gas chromatographer and mass spectrometer using a Perkin-Elmer Elite fused silica column (length: $30 \mathrm{~m}$, diameter: $0.32 \mathrm{~mm}$ ) and Helium as carrier gas. High resolution mass spectrometric measurements were performed by the mass spectrometry service of ISIC at the EPFL on a MICROMASS (ESI) Q-TOF Ultima API. HPLC measurement were done on a JASCO HPLC system with an AS2055 Autosampler, a PU 2089 Pump, a UV 2075 detector and a SEDEX 85 (SEDERE) detector using a CHIRALPAK IC, IB or IA column from DAICEL Chemical Industries Ltd. HPLC grade solvents from Sigma-Aldrich were used.

1-Phenyl-1-trimethylsiloxyethylene (5c) was purchased from Alfa Aesar [13735-81-4]

Vinyloxytrimethylsilane (5p) was purchased from Alfa Aesar [6213-94-1] 


\section{Preparation of Substrates}

\section{Synthesis of aminocyclopropane}

\section{$\underline{\text { Tosyl azide (11): }}$}

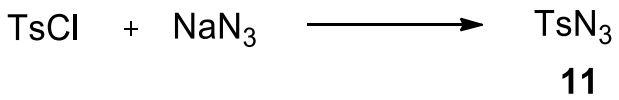

Following a reported procedure ${ }^{1}$, a solution of sodium azide $(10.3 \mathrm{~g}, 157 \mathrm{mmol}, 1.5 \mathrm{eq})$ in water (60 $\mathrm{ml})$ was added dropwise over $1 \mathrm{~h}$ to a solution of tosyl chloride $(20 \mathrm{~g}, 0.11 \mathrm{~mol}, 1 \mathrm{eq})$ in acetone (200 $\mathrm{ml})$ at $0^{\circ} \mathrm{C}$. The reaction was allowed to warm up to $23^{\circ} \mathrm{C}$ and stirred for $16 \mathrm{~h}$. The acetone was removed under reduced pressure at $25{ }^{\circ} \mathrm{C}$ and the reaction mixture was extracted with ether $(2 \mathrm{x} 100$ $\mathrm{ml})$. The combined organic layers were washed with water $(2 \times 100 \mathrm{ml}), 5 \% \mathrm{Na}_{2} \mathrm{CO}_{3}(2 \times 100 \mathrm{ml})$ and water $(2 \times 100 \mathrm{ml})$, dried over $\mathrm{MgSO}_{4}$ and the solvent was removed under reduced pressure to afford tosyl azide $(20.7 \mathrm{~g}, 107 \mathrm{mmol}$, quantitative $)$ as a colorless oil which solidify under storage at $4^{\circ} \mathrm{C}$.

${ }^{1} \mathbf{H}$ NMR $\left(400 \mathrm{MHz}, \mathrm{CDCl}_{3}\right) \delta: 7.87(\mathrm{~d}, J=8.4 \mathrm{~Hz}, 2 \mathrm{H}, \mathrm{Ar} \mathrm{H}), 7.43(\mathrm{~d}, J=8.4 \mathrm{~Hz}, 2 \mathrm{H}, \mathrm{Ar} \mathrm{H}), 2.51$ (s, $3 \mathrm{H}, \mathrm{CH}_{3}$ ).

The characterization data for $\mathbf{1 1}$ corresponded to the reported values ${ }^{1}$

\section{Dimethyl 2-diazomalonate (12):}

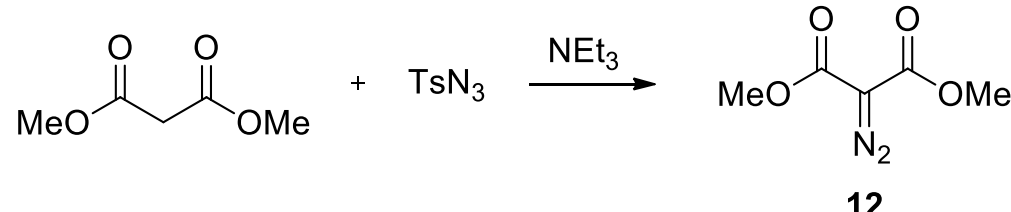

Following a reported procedure ${ }^{2}$, dimethylmalonate $(7.93 \mathrm{~mL}, 69.7 \mathrm{mmol}, 1 \mathrm{eq})$, triethylamine (10.6 $\mathrm{mL}, 76.6 \mathrm{mmol}, 1.1 \mathrm{eq})$ and tosyl azide $(15.1 \mathrm{~g}, 76.6 \mathrm{mmol}, 1.1 \mathrm{eq})$ were dissolved in acetonitrile (100 $\mathrm{mL})$. The solution was stirred at $23^{\circ} \mathrm{C}$ for $20 \mathrm{~h}$. The solution was concentrated under reduced pressure and partitioned between dichloromethane and water. The layers were separated and the aqueous layer was extracted with DCM (x1). The organic layers were combined and dried over $\mathrm{MgSO}_{4}$. The crude was first filtered over a plug of silica gel $\left(\mathrm{PET} / \mathrm{Et}_{2} \mathrm{O} 1 / 1\right)$ to remove most of the tosylamide formed during the reaction. Then, purification by column chromatography $\left(\mathrm{PET}_{\mathrm{Et}} \mathrm{Et}_{2} \mathrm{O} 1 / 1\right)$ afforded dimethyl 2-diazomalonate $(10.4 \mathrm{~g}, 65.5 \mathrm{mmol}, 94 \%)$ as a yellow oil which solidified under storage at $4^{\circ} \mathrm{C}$.

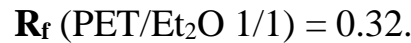

${ }^{1} \mathbf{H}$ NMR $\left(400 \mathrm{MHz}, \mathrm{CDCl}_{3}\right) \delta: 3.87(\mathrm{~s}, 1 \mathrm{H})$.

${ }^{13} \mathbf{C}$ NMR $\left(101 \mathrm{MHz}, \mathrm{CDCl}_{3}\right) \delta 161.2,52.4^{3}$

The characterization data for $\mathbf{1 2}$ corresponded to the reported values ${ }^{2}$

\footnotetext{
${ }^{1}$ P. R. Serwinski, B. Esat, P. M. Lahti, Y. Liao, R. Walton, J. Lan, J. Org. Chem. 2004, 69, 5247

${ }^{2}$ P. Wyatt, A. Hudson, J. Charmant, A. G. Orpen, H. Phetmung, Org. Biomol. Chem. 2006, 4, 2218

${ }^{3}$ The diazo carbon could not be detected
} 


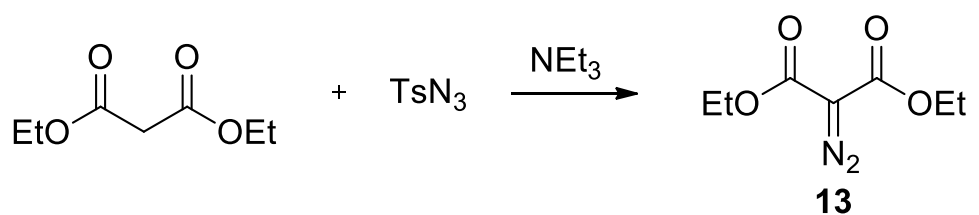

Following the procedure described below, diethylmalonate $(2.17 \mathrm{~mL}, 14.3 \mathrm{mmol}, 1 \mathrm{eq})$, triethylamine $(2.18 \mathrm{~mL}, 15.7 \mathrm{mmol}, 1.1 \mathrm{eq})$ and tosyl azide $(3.09 \mathrm{~g}, 15.7 \mathrm{mmol}, 1.1 \mathrm{eq})$ were dissolved in acetonitrile $(20 \mathrm{~mL})$. The solution was stirred at $23^{\circ} \mathrm{C}$ for $20 \mathrm{~h}$. The solution was concentrated under reduced pressure and partitioned between DCM and water. The layers were separated and the aqueous layer was extracted with dichloromethane (x1). The organic layers were combined and dried over $\mathrm{MgSO}_{4}$. The crude was first filtered over a plug of silica gel $\left(\mathrm{PET} / \mathrm{Et}_{2} \mathrm{O}\right.$ 1/1) to remove most of the tosylamide formed during the reaction. Then, purification by column chromatography $\left(\mathrm{PET}_{2} \mathrm{Et}_{2} \mathrm{O} 1 / 1\right)$ afforded dimethyl 2-diazomalonate $(2.3 \mathrm{~g}, 12 \mathrm{mmol}, 86 \%)$ as a yellow oil which solidified under storage at $4^{\circ} \mathrm{C}$.

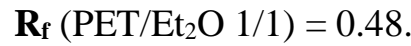

${ }^{1} \mathbf{H}$ NMR $\left(400 \mathrm{MHz}, \mathrm{CDCl}_{3}\right) \delta 4.27$ (q, $\left.4 \mathrm{H}, J=7.1 \mathrm{~Hz}\right), 1.28(\mathrm{t}, 6 \mathrm{H}, J=7.1 \mathrm{~Hz})$.

${ }^{13} \mathbf{C}$ NMR $\left(101 \mathrm{MHz}, \mathrm{CDCl}_{3}\right) \delta 161.0,61.5,14.3^{3}$

The characterization data for $\mathbf{1 3}$ corresponded to the reported values ${ }^{4}$

\section{Cyclopropanes 4 a-f}

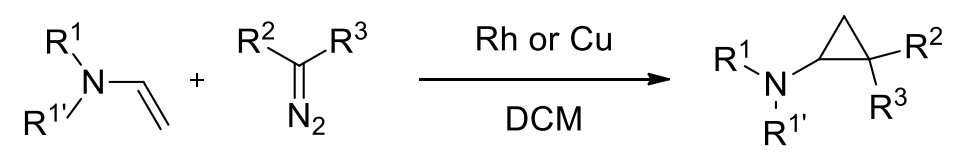

\section{Rhodium catalyzed cyclopropanation:}

\section{Dimethyl 2-(1,3-dioxoisoindolin-2-yl)cyclopropane-1,1-dicarboxylate (4f):}<smiles>COC(=O)C1(C(C)OC)CC1Nc1ccccc1</smiles>

Following a modified procedure ${ }^{5}$, bis[rhodium( $\alpha, \alpha, \alpha^{\prime}, \alpha^{\prime}$-tetramethyl-1,3-benzenedipropionic acid)] (10.9 $\mathrm{mg}, 0.014 \mathrm{mmol}, 0.1 \mathrm{~mol} \%$ ) is weighted in the glovebox. The flask is closed with a septum and put under $\mathrm{N}_{2}$ atmosphere. A solution of $N$-vinyl-phthalimide $(2.5 \mathrm{~g}, 14 \mathrm{mmol}, 1 \mathrm{eq})$ in $30 \mathrm{~mL}$ of dry dichloromethane is added and the resulting green suspension is cooled down to $0^{\circ} \mathrm{C}$ with an ice/water bath. A solution of dimethyl-2-diazomalonate $(2.5 \mathrm{~g}, 15 \mathrm{mmol}, 1.1 \mathrm{eq})$ in $20 \mathrm{~mL}$ of dichloromethane is added over five minutes. When the addition is complete, the reaction is allowed to warm to room temperature. After $5 \mathrm{~h}$ at room temperature, the solvent is removed under reduced pressure and the

\footnotetext{
${ }^{4}$ M. Kitamura, N. Tashiro, S. Miyagawa, T. Okauchi, Synthesis, 2011, 7, 1037

${ }^{5}$ F. Gonzalez-Bobes, M. D. B. Fenster, S. Kiau, L. Kolla, S. Kolotuchin, M. Soumeillant, Adv. Synth. Catal. 2008, 350, 813
} 
crude is directly purified by column chromatography (9:1 Hexane/Ethyl Acetate to 7:3 Hexane/Ethyl Acetate). $3.40 \mathrm{~g}$ (11.2 $\mathrm{mmol}, 78 \%$ yield) of $\mathbf{4 f}$ as a white solid was isolated.

The two enantiomers were separated by HPLC using Chiralpack IA column $(0.46 \times 25 \mathrm{~cm}), 95: 5$ Hexane/Isopropanol, $1 \mathrm{ml} / \mathrm{min} ; \operatorname{tr}_{1}=23.9 \mathrm{~min},[\alpha]_{\mathrm{D}}{ }^{25.0} 122\left(\mathrm{c}=0.69, \mathrm{CHCl}_{3}\right) ; \operatorname{tr}_{2}=27.1 \mathrm{~min}$,

$[\alpha]_{D}{ }^{25.0}-122\left(\mathrm{c}=0.1, \mathrm{CHCl}_{3}\right)$

Preparative HPLC: Column IA 20x250 mm, 98:2 Hexane/Isopropanol, $16 \mathrm{ml} / \mathrm{min} . \operatorname{tr}_{1}=45 \mathrm{~min}, \operatorname{tr}_{2}=$ 75 min

$\mathbf{R}_{\mathbf{f}} 0.27$ (6:4, Hexane/Ethyl acetate).

Mp 124.6- $125^{\circ} \mathrm{C}$

${ }^{1} \mathbf{H}$ NMR $\left(400 \mathrm{MHz}, \mathrm{CDCl}_{3}\right) \delta 7.86(\mathrm{~m}, 2 \mathrm{H}, P h t h), 7.75(\mathrm{~m}, 2 \mathrm{H}, P h t h), 3.85$ (s, $\left.3 \mathrm{H}, O M e\right), 3.72(\mathrm{dd}$, $1 \mathrm{H}, J=8.5,6.6 \mathrm{~Hz}, \mathrm{~N}-\mathrm{CH}$ ), $3.64\left(\mathrm{~s}, 3 \mathrm{H}, \mathrm{OMe}\right.$ ), 2.73 (dd, $1 \mathrm{H}, J=6.5,6.5 \mathrm{~Hz}, \mathrm{CH}_{2}$ ), 2.06 (dd, 1 $\left.\mathrm{H}, J=8.5,6.4 \mathrm{~Hz}, \mathrm{CH}_{2}\right)$.

${ }^{13}$ C NMR $\left(101 \mathrm{MHz}, \mathrm{CDCl}_{3}\right) \delta 168.5,167.8,166.9,134.3,131.4,123.5,53.1,53.0,34.9,33.1,19.6$.

IR 2956 (w), 1783 (w), 1727 (s), 1468 (w), 1439 (w), 1399 (m), 1329 (m), 1294 (m), 1222 (m), 1134 (w), $909(\mathrm{w}), 876(\mathrm{w}), 720(\mathrm{~m})$.

HRMS (ESI) calcd for $\mathrm{C}_{15} \mathrm{H}_{14} \mathrm{NO}_{6}{ }^{+}[\mathrm{M}+\mathrm{H}]^{+} 304.0816$; found 304.0804 .

Trans-ethyl 2-(((benzyloxy)carbonyl)amino)cyclopropanecarboxylate (4a):<smiles>CCOC[C@@H]1C[C@H]1NC(=O)OCc1ccccc1</smiles>

Following the same procedure described above, using $50 \mathrm{mg}(0.28 \mathrm{mmol}, 1 \mathrm{eq})$ of $N$-Vinyl- $O$-benzyl Urethane $^{6}, 64 \mathrm{mg}(0.56 \mathrm{mmol}, 2 \mathrm{eq})$ of Ethyl-diazoacetate and $2.1 \mathrm{mg}(0.0028 \mathrm{mmol}, 1 \mathrm{~mol} \%)$ of $\operatorname{Bis}\left[\right.$ rhodium $\left(\alpha, \alpha, \alpha^{\prime}, \alpha^{\prime}\right.$-tetramethyl-1,3-benzenedipropionic acid)]. After column chromatography $\left(100 \%\right.$ Heptane $+1 \% \mathrm{Net}_{3}$ to $9: 1 \mathrm{Heptane} /$ Ethyl Acetate $\left.+1 \% \mathrm{Net}_{3}\right) 26.7 \mathrm{mg}(0.10 \mathrm{mmol}, 36 \%$ yield $)$ of trans-product is obtained.

$\mathbf{R}_{\mathbf{f}} 0.5$ (7:3 Petroleum ether/Ethyl Acetate)

${ }^{1} \mathbf{H}$ NMR $\left(400 \mathrm{MHz}, \mathrm{CDCl}_{3}\right) \delta$ 7.42-7.32 (m, $\left.\left.5 \mathrm{H}, \mathrm{Ar}\right), 5.13\left(\mathrm{~m}, 2 \mathrm{H}, \mathrm{Ar}-\mathrm{CH}_{2}\right)\right), 4.16$ (m, $2 \mathrm{H}, \mathrm{O}-\mathrm{CH}_{2}-$ $\mathrm{CH}_{3}, 3.12(\mathrm{~m}, 1 \mathrm{H}, \mathrm{N}-\mathrm{C}-\mathrm{H}), 1.79\left(\mathrm{~m}, 1 \mathrm{H}, \mathrm{CH}_{2}\right), 1.45\left(\mathrm{~m}, 1 \mathrm{H}, \mathrm{CH}_{2}\right), 1.25-1.31$ (t, $3 \mathrm{H}, \mathrm{J}=7.5 \mathrm{~Hz}, \mathrm{O}-$ $\left.\mathrm{CH}_{2}-\mathrm{CH}_{3}\right), 1.14-1.19\left(\mathrm{~m}, 1 \mathrm{H}, \mathrm{CH}_{2}\right)$

The characterization data for $\mathbf{4 a}$ corresponded to the reported values ${ }^{7}$

Diethyl 2-(1,3-dioxoisoindolin-2-yl)cyclopropane-1,1-dicarboxylate (4d):<smiles>CCOC(=O)C1(C(=O)OCC)CC1Nc1ccccc1</smiles>

Following the same procedure described above, using $3.0 \mathrm{~g}(18 \mathrm{mmol}, 1 \mathrm{eq})$ of $N$-vinyl-phthalimide, $4.0 \mathrm{~g}(21 \mathrm{mmol}, 1.2 \mathrm{eq})$ of diethyl-2-diazomalonate and $14 \mathrm{mg}(0.018 \mathrm{mmol}, 0.1 \mathrm{~mol} \%)$ of $\operatorname{Bis}\left[\right.$ rhodium( $\alpha, \alpha, \alpha^{\prime}, \alpha^{\prime}$-tetramethyl-1,3-benzenedipropionic acid)], $5.4 \mathrm{~g}$ (16 mmol, $90 \%$ yield) of a white solid was obtained.

$\mathbf{R}_{\mathbf{f}} 0.36$ (6:4, Hexane/Ethyl acetate)

${ }^{6}$ D. J. am Ende, K. M. DeVries, P. J. Clifford, and S. J. Brenek, Org. Proc. Res. Dev., 1998, 2, 382

${ }^{7}$ J. A. Miller, E. J. Hennessy, W. J. Marshall, M. A. Scialdone, S. T. Nguyen, J. Org. Chem., 2003, 68, 7884 
Mp $93.1^{\circ} \mathrm{C}$.

${ }^{1} \mathbf{H}$ NMR $\left(400 \mathrm{MHz}, \mathrm{CDCl}_{3}\right) \delta 7.85\left(\mathrm{~m}, 2 \mathrm{H}\right.$, Phth), $7.74\left(\mathrm{~m}, 2 \mathrm{H}\right.$, Phth), $4.30\left(\mathrm{~m}, 2 \mathrm{H}, \mathrm{OCH}_{2}\right), 4.07$ $\left(\mathrm{m}, 2 \mathrm{H}, O_{C H}\right), 3.71(\mathrm{dd}, 1 \mathrm{H}, J=8.5,6.6 \mathrm{~Hz}, \mathrm{~N}-\mathrm{C}-\mathrm{H}), 2.74\left(\mathrm{t}, 1 \mathrm{H}, J=6.5 \mathrm{~Hz}, \mathrm{CH}_{2}\right), 2.02$ (dd, $1 \mathrm{H}, J$ $\left.=8.5,6.4 \mathrm{~Hz}, \mathrm{CH}_{2}\right), 1.34\left(\mathrm{t}, 3 \mathrm{H}, J=7.1 \mathrm{~Hz}, \mathrm{CH}_{3}\right), 1.12\left(\mathrm{t}, 3 \mathrm{H}, J=7.1 \mathrm{~Hz}, \mathrm{CH}_{3}\right)$.

${ }^{13} \mathrm{C}$ NMR $\left(101 \mathrm{MHz}, \mathrm{CDCl}_{3}\right) \delta 168.2,167.8,166.4,134.3,131.6,123.4,62.0,61.8,34.7,33.5,19.2$, 14.1, 13.8 .

IR 2985 (w), 2938 (w), 2907 (w), 1783 (m), 1719 (s), 1614 (w), 1393 (s), 1321 (m), 1218 (s), 1133 (m), 719 (s).

HRMS (ESI) calcd for $\mathrm{C}_{17} \mathrm{H}_{18} \mathrm{NO}_{6}{ }^{+}[\mathrm{M}+\mathrm{H}]^{+} 332.1129$; found 332.1135

\section{Copper catalyzed cyclopropanation}

Trans-ethyl 2-(1,3-dioxoisoindolin-2-yl)cyclopropanecarboxylate (4e):<smiles>CCOC(=O)[C@H]1C[C@H]1Nc1ccccc1</smiles>

copper-(I)-trifluoromethanesulfonate-toluene complex (26.4 $\mathrm{mg}, 0.0510 \mathrm{mmol}, 2.5 \mathrm{~mol} \%)$ is weighted in the glovebox. The flask is closed with a septum, protected from light and put under $\mathrm{N}_{2}$ atmosphere. A solution of $N$-vinyl-phthalimide $(500 \mathrm{mg}, 2.04 \mathrm{mmol}, 1 \mathrm{eq}$ ) in $4 \mathrm{~mL}$ of dry dichloromethane is added. Ethyl diazoacetate $(0.860 \mathrm{ml}, 8.16 \mathrm{mmol}, 4 \mathrm{eq})$ is added via a syringe pump over $18 \mathrm{~h}$. When the addition is complete, the solvent is removed under reduced pressure and the crude is directly purified by column chromatograpy (8:2 Petroleum Ether/Ethyl Acetate). The isolated yellow solid is recrystallized in Petroleum Ether/Ethyl Acetate to give $205 \mathrm{mg}$ (0.791 mmol, $40 \%$ yield) of the trans cyclopropane as a colorless solid.

$\mathbf{R}_{\mathbf{f}} 0.36$ (8:2, Petroleum Ether/Ethyl acetate).

${ }^{1} \mathbf{H}$ NMR $\left(400 \mathrm{MHz}, \mathrm{CDCl}_{3}\right) \delta$ 7.87-7.81 (m, $2 \mathrm{H}$, Phth), 7.77-7.71 (m, $2 \mathrm{H}$, Phth), 4.22 (q, $2 \mathrm{H}, J=$ $\left.7.2 \mathrm{~Hz}, \mathrm{O}-\mathrm{CH}_{2}-\mathrm{CH}_{3}\right), 3.35-3.28(\mathrm{~m}, 1 \mathrm{H}, \mathrm{N}-\mathrm{C}-\mathrm{H}), 2.25-2.20\left(\mathrm{~m}, 1 \mathrm{H}, \mathrm{CHCO}_{2} \mathrm{Et}\right), 1.76$ (dt, $1 \mathrm{H}, J=9.3$, $\left.5.5 \mathrm{~Hz}, \mathrm{CH}_{2}\right), 1.65\left(\mathrm{dt}, 1 \mathrm{H}, J=8.1,5.9 \mathrm{~Hz}, \mathrm{CH}_{2}\right), 1.32\left(\mathrm{t}, 3 \mathrm{H}, J=7.2 \mathrm{~Hz}, \mathrm{O}-\mathrm{CH}_{2}-\mathrm{CH}_{3}\right)$.

${ }^{13} \mathbf{C ~ N M R}\left(101 \mathrm{MHz}, \mathrm{CDCl}_{3}\right) \delta 172.1,168.0,134.3,131.6,123.4,61.1,29.5,20.0,14.2,13.6$

The characterization data for $\mathbf{4 b}$ corresponded to the reported values ${ }^{8}$

\section{Trans-ethyl 2-(2-oxopyrrolidin-1-yl)cyclopropanecarboxylate (4b):}<smiles>CCOC[C@H]1C[C@@H]1N1CCCC1=O</smiles>

Following the same procedure described above, using $250 \mathrm{mg}$ ( $2.25 \mathrm{mmol}, 1 \mathrm{eq})$ of commercially available $N$-vinyl-pyrrolidone [88-12-0], $0.95 \mathrm{~mL}$ ( $8.9 \mathrm{mmol}, 4 \mathrm{eq})$ of ethyl diazoacetate and $29 \mathrm{mg}$ (0.056 mmol, $2.5 \mathrm{~mol} \%)$ of copper-(I)-trifluoromethanesulfonate-toluene complex, $240 \mathrm{mg}$ (1.22

\footnotetext{
${ }^{8}$ A. Abu-Elfotoh, K. Phomkeona, K. Shibatomi, S. Iwasa, Angew. Chem. Int. Ed. 2010, 49, 8439 -8443
} 
mmol, $54 \%$ yield) of a yellow oil is obtained after column chromatography purification (9/1 Petroleum Ether/Ethyl Acetate to 100 Ethyl Acetate).

$\mathbf{R}_{\mathbf{f}} 0.2$ (Ethyl acetate).

${ }^{1} \mathbf{H}$ NMR $\left(400 \mathrm{MHz}, \mathrm{CDCl}_{3}\right) \delta 4.15\left(\mathrm{qd}, 2 \mathrm{H}, J=7.2,1.5 \mathrm{~Hz}, \mathrm{O}-\mathrm{CH}_{2}-\mathrm{CH}_{3}\right), 3.31(\mathrm{t}, 2 \mathrm{H}, J=7.2 \mathrm{~Hz}$, $\mathrm{CH}_{2}$ pyrrolidone), 3.20-3.15 (m, $\left.1 \mathrm{H}, \mathrm{N}-\mathrm{C}-\mathrm{H}\right), 2.39$ (t, $2 \mathrm{H}, \mathrm{J}=8.0 \mathrm{~Hz}, \mathrm{CH}_{2}$ pyrrolidone), 2.05-1.96 (m, $2 \mathrm{H}, \mathrm{CH}_{2}$ pyrrolidone), 1.88-1.82 (m, $\left.1 \mathrm{H}, \mathrm{CHCO}_{2} \mathrm{Et}\right), 1.51-1.38\left(\mathrm{~m}, 2 \mathrm{H}, \mathrm{CH}_{2}\right), 1.27(\mathrm{t}, 3 \mathrm{H}, J=7.1$ $\left.\mathrm{Hz}, \mathrm{O}-\mathrm{CH}_{2}-\mathrm{CH}_{3}\right)$.

${ }^{13} \mathrm{C}$ NMR $\left(101 \mathrm{MHz}, \mathrm{CDCl}_{3}\right) \delta 175.9,172.3,60.9,47.2,34.1,31.7,19.7,18.0,14.2,14.1$.

The characterization data for $\mathbf{4 b}$ corresponded to the reported values ${ }^{8}$

\section{Ethyl 2-(2-oxooxazolidin-3-yl)cyclopropanecarboxylate (4c):}<smiles>CCOC1CC1N1CCOC1=O</smiles>

Following the same procedure described above, using $2.42 \mathrm{~g}(21.4 \mathrm{mmol}, 1 \mathrm{eq})$ of $N$-vinyloxazolidinone $^{9}, 9.0 \mathrm{~mL}$ ( $\left.86 \mathrm{mmol}, 4 \mathrm{eq}\right)$ of ethyl diazoacetate and $277 \mathrm{mg}(0.534 \mathrm{mmol}, 2.5 \mathrm{~mol} \%)$ of copper-(I)-trifluoromethanesulfonate-toluene complex, $3.21 \mathrm{~g}$ (16.1 mmol, $75 \%$ yield) of a yellow oil is obtained after column chromatography purification (9/1 Petroleum Ether/Ethyl Acetate to 100 Ethyl Acetate).

1:1 Mixture of cis and trans non-separable diastereomers.

$\mathbf{R}_{\mathbf{f}} 0.3(98: 2 \mathrm{DCM} / \mathrm{MeOH})$.

${ }^{1} \mathbf{H}$ NMR (400 MHz, $\mathrm{CDCl}_{3}$ ) $\delta$ 4.35-4.26 (m, $4 \mathrm{H}, \mathrm{CH}_{2}$ oxazolidinone), 4.19-4.11 (m, $4 \mathrm{H}, \mathrm{O}-\mathrm{CH}_{2^{-}}$ $\mathrm{CH}_{3}$ ), $3.74\left(\mathrm{dd}, 1 \mathrm{H}, \mathrm{J}=7.9,7.9 \mathrm{~Hz}, \mathrm{~N}-\mathrm{C}-\mathrm{H}\right.$ ), 3.60-3.50 (m, $3 \mathrm{H}, \mathrm{N}-\mathrm{C}-\mathrm{H}$ and $\mathrm{CH}_{2}$ oxazolidinone), $3.07-$ $3.00\left(\mathrm{~m}, 2 \mathrm{H}, \mathrm{CH}_{2}\right.$ oxazolidinone), $2.02\left(\mathrm{~m}, 1 \mathrm{H}, \mathrm{CHCO}_{2} \mathrm{Et}\right), 1.90\left(\mathrm{~m}, 1 \mathrm{H}, \mathrm{CHCO}{ }_{2} \mathrm{Et}\right), 1.55(\mathrm{q}, 1 \mathrm{H}, J$ $\left.=6.0 \mathrm{~Hz}, \mathrm{CH}_{2}\right), 1.52-1.43\left(\mathrm{~m}, 2 \mathrm{H}, \mathrm{CH}_{2}\right), 1.38-1.31\left(\mathrm{~m}, 1 \mathrm{H}, \mathrm{CH}_{2}\right), 1.30-1.24\left(\mathrm{~m}, 6 \mathrm{H}, \mathrm{O}-\mathrm{CH}_{2}-\mathrm{CH}_{3}\right)$. ${ }^{13} \mathrm{C} \mathrm{NMR}\left(101 \mathrm{MHz}, \mathrm{CDCl}_{3}\right) \delta 171.9,170.5,158.5,157.9,62.3,61.9,61.0,61.0,45.7,45.7,34.4$, $33.0,20.7,20.6,15.0,14.2,12.8^{10}$

IR 2986 (w), 2985 (w), 2917 (w), 1751 (s), 1723 (s), 1722 (s), 1426 (m), 1409 (m), 1185 (s), 1040 (m).

HRMS (ESI) calcd for C9H14NO4+ [M+H]+ 200.0917; found 200.0908

\footnotetext{
${ }^{9}$ Prepared following the reported procedure: Org. Lett. 2004, 6, 1845

${ }^{10}$ One carbon aliphatic of one diastereomer could not be resolved
} 


\section{Synthesis of Silyl Enol Ethers}

\section{General procedure for the synthesis of Silyl Enol Ethers:}<smiles>[R]C(=O)C([R])([R])[R]</smiles><smiles>[R3][SiH2]OC([R])=C([R])[R]</smiles>

\section{Method A:}

The ketone (1 eq) in anhydrous THF is added in an oven-dried flask sealed with a septum and under $\mathrm{N}_{2}$ atmosphere. The solution is cooled down to $-78{ }^{\circ} \mathrm{C}$ and a $2 \mathrm{M}$ solution of NaHMDS (1.22 eq) is added dropwise. The cold bath is removed and the pale yellow solution is stirred for 1 hour at room temperature. The reaction is cooled again at $-78{ }^{\circ} \mathrm{C}$ and the corresponding silyl chloride $(1.2 \mathrm{eq})$ is added dropwise. The reaction is stirred at room temperature for 5 hours after what the solvent is directly removed under reduced pressure. The resulting orange oil is purified by plug or by column chromatography on triethylamine-deactivated silica (100\% Hexane).

\section{Method B:}

The ketone (1 eq) in anhydrous dichloromethane is added in an oven-dried flask sealed with a septum and under $\mathrm{N}_{2}$ atmosphere . Triethylamine (1.8 eq) and then triisopropylsilyl-trifluoromethanesulfonate (1.2 eq) are added at room temperature. After two hours, the reaction is diluted with dichloromethane and washed with a solution of sat. $\mathrm{NH}_{4} \mathrm{Cl}$. The aqueous layer is extracted three times with ethyl acetate. The organic layers are collected, washed with brine, dried over anhydrous sodium sulfate, filtered and concentrated under reduced pressure. The crude oil is purified by plug or column chromatography on triethylamine deactivated silica (100\% Hexane).

\section{Triisopropyl((1-phenylvinyl)oxy)silane (5a):}<smiles>C=C(O[Si](C(C)C)(C(C)C)C(C)C)c1ccccc1</smiles>

Using method A, starting from $580 \mathrm{mg}$ of acetophenone (4.82 mmol), $1.03 \mathrm{~g}(3.72 \mathrm{mmol}, 77 \%$ yield) of a colorless oil was obtained.

${ }^{1} \mathbf{H}$ NMR $\left(400 \mathrm{MHz}, \mathrm{CDCl}_{3}\right) \delta$ 7.69-7.65 (m, $\left.2 \mathrm{H}, \mathrm{Ar}\right), 7.38-7.29(\mathrm{~m}, 3 \mathrm{H}, \mathrm{Ar}), 4.85(\mathrm{~d}, 1 \mathrm{H}, J=1.8$ $\left.\mathrm{Hz}, \mathrm{C}=\mathrm{CH}_{2}\right), 4.41\left(\mathrm{~d}, 1 \mathrm{H}, J=1.8 \mathrm{~Hz}, \mathrm{C}=\mathrm{CH}_{2}\right), 1.39-1.27\left(\mathrm{~m}, 3 \mathrm{H}, \mathrm{SiCH}\left(\mathrm{CH}_{3}\right)_{2}\right), 1.19-1.13(\mathrm{~m}, 18 \mathrm{H}$, $\left.\mathrm{SiCH}\left(\mathrm{CH}_{3}\right)_{2}\right)$.

${ }^{13} \mathbf{C}$ NMR $\left(101 \mathrm{MHz}, \mathrm{CDCl}_{3}\right) \delta 156.2,138.0,128.2,128.1,125.4,90.0,18.2,12.9$

The characterization data for $5 \mathbf{a}$ corresponded to the reported values ${ }^{11}$

\footnotetext{
${ }^{11}$ Jun-Feng Zhao, Boon-Hong Tan and Teck-Peng Loh Chem. Sci. 2011, 2, 349
} 


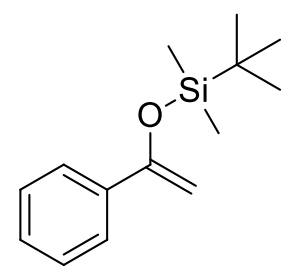

Using method A, starting from $580 \mathrm{mg}$ of acetophenone (4.82 mmol), $960 \mathrm{mg}(4.10 \mathrm{mmol}, 85 \%$ yield) of a colorless oil was obtained.

${ }^{1} \mathbf{H}$ NMR $\left(400 \mathrm{MHz}, \mathrm{CDCl}_{3}\right) \delta$ 7.65-7.60 (m, $\left.2 \mathrm{H}, A r\right), 7.39-7.29(\mathrm{~m}, 3 \mathrm{H}, \mathrm{Ar}), 4.89(\mathrm{~d}, 1 \mathrm{H}, J=1.7$ $\left.\mathrm{Hz}, \mathrm{C}=\mathrm{CH})_{2}\right), 4.42\left(\mathrm{~d}, 1 \mathrm{H}, J=1.7 \mathrm{~Hz}, \mathrm{C}=\mathrm{CH}_{2}\right), 1.00\left(\mathrm{~s}, 9 \mathrm{H}, \mathrm{Si}\left(\mathrm{CH}_{3}\right)_{2} \mathrm{C}\left(\mathrm{CH}_{3}\right)_{3}\right), 0.21(\mathrm{~s}, 5 \mathrm{H}$, $\left.\mathrm{Si}\left(\mathrm{CH}_{3}\right)_{2} \mathrm{C}\left(\mathrm{CH}_{3}\right)_{3}\right)$.

${ }^{13} \mathbf{C ~ N M R}\left(101 \mathrm{MHz}, \mathrm{CDCl}_{3}\right) \delta 156.0,137.8,128.2,128.1,125.3,90.9,25.9,18.4,-4.6$.

The characterization data for $\mathbf{5 b}$ corresponded to the reported values ${ }^{11}$

\section{$\underline{\text { (1-(4-Fluorophenyl)vinyl)oxy)triisopropylsilane (5d): }}$}<smiles>C=C(O[Si](C(C)C)(C(C)C)C(C)C)c1ccc(F)cc1</smiles>

Using method B, starting from $500 \mathrm{mg}$ of 4'-Fluoroacetophenone (3.62 mmol), $776 \mathrm{mg}$ (2.64 mmol, 73 $\%$ yield) of a colorless oil were obtained.

${ }^{1} \mathbf{H}$ NMR $\left(400 \mathrm{MHz}, \mathrm{CDCl}_{3}\right) \delta 7.61(\mathrm{~m}, 2 \mathrm{H}, \mathrm{Ar}), 7.00(\mathrm{~m}, 2 \mathrm{H}, \mathrm{Ar}), 4.77\left(\mathrm{~d}, 1 \mathrm{H}, J=2.0 \mathrm{~Hz}, \mathrm{C}=\mathrm{CH}_{2}\right)$, $4.39\left(\mathrm{~d}, 1 \mathrm{H}, J=1.9 \mathrm{~Hz}, \mathrm{C}=\mathrm{CH}_{2}\right), 1.43-1.29\left(\mathrm{~m}, 3 \mathrm{H}, \mathrm{SiCH}\left(\mathrm{CH}_{3}\right)_{2}\right), 1.22-1.14\left(\mathrm{~m}, 18 \mathrm{H}, \mathrm{SiCH}\left(\mathrm{CH}_{3}\right)_{2}\right)$.

${ }^{13} \mathbf{C}$ NMR $\left(101 \mathrm{MHz}, \mathrm{CDCl}_{3}\right) \delta 162.8(\mathrm{~d}, J=248 \mathrm{~Hz}), 155.3,134.1(\mathrm{~d}, J=3 \mathrm{~Hz}), 127.1(\mathrm{~d}, J=8.1$ $\mathrm{Hz}), 114.9(\mathrm{~d}, J=21.5 \mathrm{~Hz}), 89.7(\mathrm{~d}, J=2 \mathrm{~Hz}), 18.1,12.8$.

The characterization data for $\mathbf{5 d}$ corresponded to the reported values ${ }^{11}$

\section{Methyl 4-(1-((triisopropylsilyl)oxy)vinyl)benzoate (5e):}

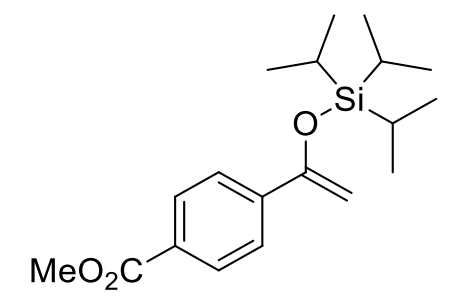

Using method B, starting from $500 \mathrm{mg}$ of methyl 4-acetylbenzoate $(2.81 \mathrm{mmol}), 856 \mathrm{mg}(2.56 \mathrm{mmol}$, $91 \%$ yield) of a colorless oil were obtained.

$\mathbf{R}_{\mathbf{f}} 0.5$ (9:1, Hexane/Ethyl acetate).

${ }^{1} \mathbf{H}$ NMR $\left(400 \mathrm{MHz}, \mathrm{CDCl}_{3}\right) \delta 8.00(\mathrm{~m}, 2 \mathrm{H}, \mathrm{Ar}), 7.70(\mathrm{~m}, 2 \mathrm{H}, \mathrm{Ar}), 4.96\left(\mathrm{~d}, 1 \mathrm{H}, J=2.0 \mathrm{~Hz}, \mathrm{C}=\mathrm{CH}_{2}\right)$, $4.53\left(\mathrm{~d}, 1 \mathrm{H}, J=2.0 \mathrm{~Hz}, \mathrm{C}=\mathrm{CH}_{2}\right), 3.92\left(\mathrm{~s}, 3 \mathrm{H}, \mathrm{CO}_{2} \mathrm{CH}_{3}\right), 1.37-1.26\left(\mathrm{~m}, 3 \mathrm{H}, \mathrm{SiCH}\left(\mathrm{CH}_{3}\right)_{2}\right), 1.18-1.12$ $\left(\mathrm{m}, 18 \mathrm{H}, \mathrm{SiCH}\left(\mathrm{CH}_{3}\right)_{2}\right)$. 
${ }^{13}$ C NMR $\left(101 \mathrm{MHz}, \mathrm{CDCl}_{3}\right) \delta 166.9,155.3,142.3,129.6,129.5,125.2,92.1,52.1,18.1,12.8$

IR 3669 (w), 3522 (w), 2959 (m), 2955 (m), 2943 (m), 2867 (m), 1722 (s), 1679 (m), 1282 (s), 1113 (s)

HRMS (ESI) calcd for $\mathrm{C}_{19} \mathrm{H}_{31} \mathrm{O}_{3} \mathrm{Si}^{+}[\mathrm{M}+\mathrm{H}]^{+}$335.2037; found 335.2032

\section{4-(1-((Triisopropylsilyl)oxy)vinyl)benzonitrile (5f):}<smiles>C=C(O[Si](C(C)C)(C(C)C)C(C)C)c1ccc(C#N)cc1</smiles>

Using method B, starting from $0.50 \mathrm{mg}$ of 4-Acetylbenzonitrile $(3.4 \mathrm{mmol}), 1.0 \mathrm{~g}$ (3.3 mmol, $97 \%$ yield) of a pale orange oil were obtained.

$\mathbf{R}_{\mathbf{f}} 0.55$ (9:1, Hexane/Ethyl acetate).

${ }^{1} \mathbf{H}$ NMR $\left(400 \mathrm{MHz}, \mathrm{CDCl}_{3}\right) \delta 7.73(\mathrm{~m}, 2 \mathrm{H}, \mathrm{Ar}), 7.62(\mathrm{~m}, 2 \mathrm{H}, \mathrm{Ar}), 4.96\left(\mathrm{~d}, 1 \mathrm{H}, \mathrm{J}=2.3 \mathrm{~Hz}, \mathrm{C}=\mathrm{CH}_{2}\right)$, $4.57\left(\mathrm{~d}, 1 \mathrm{H}, J=2.3 \mathrm{~Hz}, \mathrm{C}=\mathrm{CH}_{2}\right), 1.39-1.26\left(\mathrm{~m}, 3 \mathrm{H}, \mathrm{SiCH}\left(\mathrm{CH}_{3}\right)_{2}\right), 1.18-1.12\left(\mathrm{~m}, 18 \mathrm{H}, \mathrm{SiCH}\left(\mathrm{CH}_{3}\right)_{2}\right)$.

${ }^{13} \mathrm{C}$ NMR $\left(101 \mathrm{MHz}, \mathrm{CDCl}_{3}\right) \delta 154.5,142.2,132.0,125.8,119.0,111.5,92.8,18.1,12.7$.

IR 2946 (m), 2945 (m), 2892 (w), 2868 (m), 2229 (w), 1614 (w), 1464 (w), 1317 (s), 1302 (m), 1111 (s), 1015 (s).

HRMS (ESI) calcd for $\mathrm{C}_{18} \mathrm{H}_{28} \mathrm{NOSi}^{+}[\mathrm{M}+\mathrm{H}]^{+}$302.1935; found 302.1935.

\section{Triisopropyl((1-(4-methoxyphenyl)vinyl)oxy)silane (5g):}

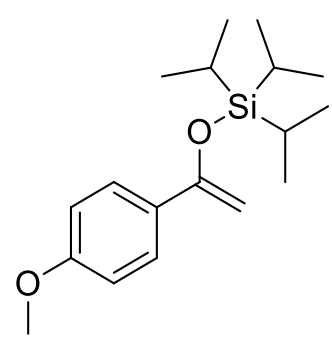

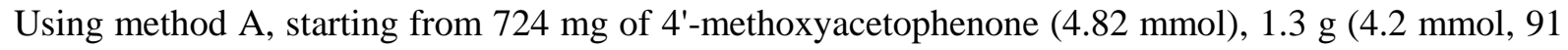
$\%$ yield) of a colorless oil were obtained.

$\mathbf{R}_{\mathbf{f}} 0.75$ (9:1, Hexane/Ethyl acetate).

${ }^{1} \mathbf{H}$ NMR $\left(400 \mathrm{MHz}, \mathrm{CDCl}_{3}\right) \delta 7.65(\mathrm{~m}, 2 \mathrm{H}, \mathrm{Ar}), 6.92(\mathrm{~m}, 2 \mathrm{H}, \mathrm{Ar}), 4.81\left(\mathrm{~d}, 1 \mathrm{H}, J=1.8 \mathrm{~Hz}, \mathrm{C}=\mathrm{CH}_{2}\right)$, $4.39\left(\mathrm{~d}, 1 \mathrm{H}, J=1.8 \mathrm{~Hz}, \mathrm{C}=\mathrm{CH}_{2}\right), 3.86\left(\mathrm{~s}, 3 \mathrm{H}, \mathrm{OCH}_{3}\right), 1.43-1.31\left(\mathrm{~m}, 3 \mathrm{H}, \mathrm{SiCH}\left(\mathrm{CH}_{3}\right)_{2}\right), 1.23-1.17(\mathrm{~m}$, $\left.18 \mathrm{H}, \mathrm{SiCH}\left(\mathrm{CH}_{3}\right)_{2}\right)$.

${ }^{13} \mathbf{C}$ NMR $\left(101 \mathrm{MHz}, \mathrm{CDCl}_{3}\right) \delta 159.7,155.9,130.7,126.7,113.4,88.4,55.2,18.2,12.9$.

IR 2944 (w), 2891 (w), 2867 (m), 2838 (w), 1608 (m), 1510 (s), 1464 (m), 1249 (s), 1175 (s), 1014 (s), 734 (s).

HRMS (ESI) calcd for $\mathrm{C}_{18} \mathrm{H}_{31} \mathrm{O}_{2} \mathrm{Si}^{+}[\mathrm{M}+\mathrm{H}]^{+}$307.2088; found 307.2076 
<smiles>C=C(O[Si](C(C)C)(C(C)C)C(C)C)c1ccccc1Br</smiles>

Using method A, starting from $0.50 \mathrm{ml}$ of 2'-Bromoacetophenone (3.7 mmol), $1.15 \mathrm{~g}$ (3.26 mmol, 88 $\%$ yield) of a colorless oil were obtained.

$\mathbf{R}_{\mathbf{f}} 0.80$ (9:1, Hexane/Ethyl acetate).

${ }^{1} \mathbf{H}$ NMR $\left(400 \mathrm{MHz}, \mathrm{CDCl}_{3}\right) \delta 7.60(\mathrm{ddd}, 1 \mathrm{H}, \mathrm{J}=8.0,1.2,0.2 \mathrm{~Hz}, A r), 7.46(\mathrm{dd}, 1 \mathrm{H}, \mathrm{J}=7.6,1.8 \mathrm{~Hz}$, $A r), 7.29(\mathrm{td}, 1 \mathrm{H}, \mathrm{J}=7.4,1.3 \mathrm{~Hz}, A r), 7.16(\mathrm{ddd}, 1 \mathrm{H}, \mathrm{J}=8.0,7.4,1.8 \mathrm{~Hz}, A r), 4.63(\mathrm{~d}, 1 \mathrm{H}, J=1.5$ $\left.\mathrm{Hz}, \mathrm{C}=\mathrm{CH}_{2}\right), 4.55\left(\mathrm{~d}, 1 \mathrm{H}, J=1.5 \mathrm{~Hz}, \mathrm{C}=\mathrm{C} H_{2}\right), 1.32-1.21\left(\mathrm{~m}, 3 \mathrm{H}, \mathrm{SiCH}\left(\mathrm{CH}_{3}\right)_{2}\right), 1.16-1.11(\mathrm{~m}, 18 \mathrm{H}$, $\left.\mathrm{SiCH}\left(\mathrm{CH}_{3}\right)_{2}\right)$.

${ }^{13} \mathrm{C}$ NMR $\left(101 \mathrm{MHz}, \mathrm{CDCl}_{3}\right) \delta 156.2,140.5,133.3,130.3,129.1,126.9,121.5,95.4,18.1,12.7$.

IR 2945 (w), 2891 (w), 2866 (m), 1705 (w), 1626 (w), 1589 (w), 1466 (m), 1317 (s), 1024 (s), 883 (m).

HRMS (ESI) calcd for $\mathrm{C}_{17}{ }^{79} \mathrm{BrH}_{28} \mathrm{OSi}^{+}[\mathrm{M}+\mathrm{H}]^{+} 355.1087$; found 355.1075

\section{Triisopropyl((1-(naphthalen-1-yl)vinyl)oxy)silane (5i):}<smiles>C=C(O[Si](C(C)C)(C(C)C)C(C)C)c1cccc2ccccc12</smiles>

Using method B, starting from $500 \mathrm{mg}$ of 1'-acetonaphthone (2.94 mmol), $865 \mathrm{mg}(2.65 \mathrm{mmol}, 91 \%$ yield) of a colorless oil were obtained.

$\mathbf{R}_{\mathbf{f}} 0.66$ (100\% Hexane).

${ }^{1} \mathbf{H}$ NMR $\left(400 \mathrm{MHz}, \mathrm{CDCl}_{3}\right) \delta$ 8.42-8.37 (m, $\left.1 \mathrm{H}, \mathrm{Ar}\right)$, 7.88-7.80 (m, $\left.2 \mathrm{H}, A r\right)$, 7.59-7.40 (m, $\left.4 \mathrm{H}, A r\right)$, $4.76\left(\mathrm{~d}, 1 \mathrm{H}, J=0.8 \mathrm{~Hz}, \mathrm{C}=\mathrm{CH}_{2}\right), 4.63\left(\mathrm{~d}, 1 \mathrm{H}, J=0.8 \mathrm{~Hz}, \mathrm{C}=\mathrm{CH}_{2}\right), 1.30-1.17\left(\mathrm{~m}, 3 \mathrm{H}, \mathrm{SiCH}\left(\mathrm{CH}_{3}\right)_{2}\right)$, 1.12-1.05 (m, $\left.18 \mathrm{H}, \mathrm{SiCH}\left(\mathrm{CH}_{3}\right)_{2}\right)$.

${ }^{13} \mathrm{C}$ NMR $\left(101 \mathrm{MHz}, \mathrm{CDCl}_{3}\right) \delta 157.6,137.7,133.7,131.0,128.4,128.1,126.6,125.9,125.8,125.7$, 125.0, 95.9, 18.0, 12.7 .

IR 2944 (m), 2892 (w), 2866 (m), 1626 (w), 1615 (w), 1464 (w), 1304 (s), 1016 (s), 778 (s).

HRMS (ESI) calcd for $\mathrm{C}_{21} \mathrm{H}_{31} \mathrm{OSi}^{+}[\mathrm{M}+\mathrm{H}]^{+} 327.2139$; found 327.2132. 


\section{(Z)-Triisopropyl((1-phenylprop-1-en-1-yl)oxy)silane (5i):}

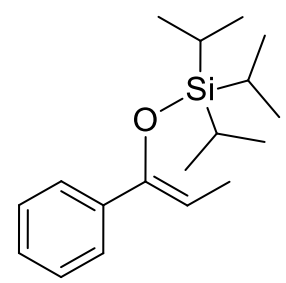

Using method A, starting from $500 \mathrm{mg}$ of propiophenone $(11.2 \mathrm{mmol}), 3.2 \mathrm{~g}(11 \mathrm{mmol}, 98 \%$ yield $)$ of a colorless oil were obtained.

$\mathbf{R}_{\mathbf{f}} 0.85$ (9:1, Hexane/Ethyl acetate).

${ }^{1} \mathbf{H}$ NMR $\left(400 \mathrm{MHz}, \mathrm{CDCl}_{3}\right) \delta$ 7.53-7.47 (m, $\left.2 \mathrm{H}, \mathrm{Ar}\right)$, 7.36-7.25 (m, $\left.3 \mathrm{H}, \mathrm{Ar}\right), 5.06(\mathrm{q}, 1 \mathrm{H}, \mathrm{J}=6.9$ $\left.\mathrm{Hz}, \mathrm{C}=\mathrm{CH}_{2}\right), 1.85-1.80$ (d, $\left.3 \mathrm{H}, J=6.9 \mathrm{~Hz}, \mathrm{CH}_{3}\right), 1.19-1.05$ (m, $21 \mathrm{H}$, TIPS).

${ }^{13} \mathrm{C}$ NMR $\left(101 \mathrm{MHz}, \mathrm{CDCl}_{3}\right) \delta 151.3,140.4,127.9,127.3,126.0,105.2,18.0,13.6,11.8$.

IR 3058 (w), 2945 (s), 2918 (m), 2867 (s), 1694 (m), 1653 (w), 1464 (m), 1325 (s), 1080 (s), 1064 (s), $883(\mathrm{~s})$.

HRMS (ESI) calcd for $\mathrm{C}_{18} \mathrm{H}_{31} \mathrm{OSi}^{+}[\mathrm{M}+\mathrm{H}]^{+}$291.2139; found 291.2145.

\section{(3,4-Dihydronaphthalen-1-yl)oxy)triisopropylsilane (5k):}<smiles>CC(C)[Si](OC1=CCCc2ccccc21)(C(C)C)C(C)C</smiles>

Using method A, starting from $540 \mathrm{mg}$ of alpha-tetralone (3.69 mmol), $973 \mathrm{mg}$ (3.22 mmol, $87 \%$ yield) of a colorless oil were obtained.

${ }^{1} \mathbf{H}$ NMR $\left(400 \mathrm{MHz}, \mathrm{CDCl}_{3}\right) \delta 7.58(\mathrm{dd}, \mathrm{J}=7.4,1.0 \mathrm{~Hz}, A r), 7.29-7.12(\mathrm{~m}, 3 \mathrm{H}, A r), 5.18(\mathrm{t}, 1 \mathrm{H}, J=$ $4.6 \mathrm{~Hz}, \mathrm{C}=\mathrm{CH}), 2.76\left(\mathrm{t}, 2 \mathrm{H}, J=7.7 \mathrm{~Hz}, \mathrm{Ar}-\mathrm{CH}_{2}\right), 2.37-2.29\left(\mathrm{~m}, 2 \mathrm{H}, \mathrm{Ar}_{-} \mathrm{CH}_{2}-\mathrm{CH}_{2}\right), 1.37-1.26(\mathrm{~m}, 3$ $\left.\mathrm{H}, \mathrm{SiCH}\left(\mathrm{CH}_{3}\right)_{2}\right), 1.19-1.13\left(\mathrm{~m}, 18 \mathrm{H}, \mathrm{SiCH}\left(\mathrm{CH}_{3}\right)_{2}\right)$.

${ }^{13} \mathrm{C}$ NMR $\left(101 \mathrm{MHz}, \mathrm{CDCl}_{3}\right) \delta 148.5,137.2,133.8,127.2,126.9,126.2,122.0,103.8,28.3,22.3$, 18.2, 12.9 .

The characterization data for $\mathbf{1 1}$ corresponded to the reported values ${ }^{12}$

${ }^{12}$ L. Zhang, J. Sun, S. A. Kozmin, Tetrahedron, 2006, 62, 11371 


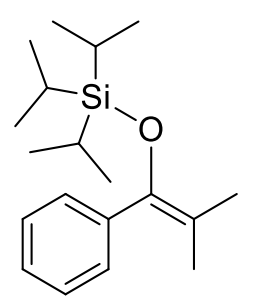

Using method A, starting from $548 \mathrm{mg}$ of propiophenone (3.70 mmol), $1.1 \mathrm{~g}(3.6 \mathrm{mmol}, 98 \%$ yield $)$ of a colorless oil were obtained.

$\mathbf{R}_{\mathbf{f}} 0.85$ (9:1, Hexane/Ethyl acetate).

${ }^{1} \mathbf{H}$ NMR $\left(400 \mathrm{MHz}, \mathrm{CDCl}_{3}\right) \delta$ 7.39-7.25 (m, $\left.5 \mathrm{H}, \mathrm{Ar}\right), 1.88\left(\mathrm{~s}, 3 \mathrm{H}, \mathrm{CH}_{3}\right), 1.63\left(\mathrm{~s}, 3 \mathrm{H}, \mathrm{CH}_{3}\right), 1.08$ $0.96(\mathrm{~m}, 21 \mathrm{H}, \mathrm{TIPS})$

${ }^{13} \mathbf{C}$ NMR $\left(101 \mathrm{MHz}, \mathrm{CDCl}_{3}\right) \delta 144.2,139.4,129.5,127.6,127.2,111.6,20.0,18.2,17.9,13.2$

IR 2966 (m), 2962 (m), 2960 (m), 2945 (m), 2924 (m), 2867 (m), 1671 (w), 1464 (w), 1162 (s), 832 (s)

HRMS (ESI) calcd for $\mathrm{C}_{19} \mathrm{H}_{33} \mathrm{OSi}^{+}[\mathrm{M}+\mathrm{H}]^{+}$305.2295; found 305.2308.

\section{$\underline{\text { (Hex-1-en-2-yloxy)triisopropylsilane (5m): }}$}

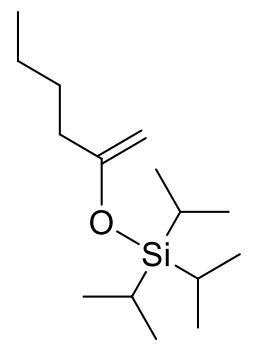

Using method A, starting from $370 \mathrm{mg}$ of 2-hexanone ( $3.69 \mathrm{mmol}), 873 \mathrm{mg}(3.40 \mathrm{mmol}, 92 \%$ yield $)$ of a colorless oil were obtained containing a 10:1.3:0.3 mixture of the di- and Z/E trisubstituted isomers.

\section{Major Isomer:}

Rf 0.75 (9:1, Hexane/Ethyl acetate)

${ }^{1} \mathbf{H}$ NMR $(400 \mathrm{MHz}, \mathrm{CDCl} 3) \delta 4.03\left(\mathrm{~s}, 1 \mathrm{H}, \mathrm{C}=\mathrm{CH}_{2}\right), 4.00\left(\mathrm{~s}, 1 \mathrm{H}, \mathrm{C}=\mathrm{CH}_{2}\right), 2.08\left(\mathrm{~m}, 2 \mathrm{H}, \mathrm{C}-\mathrm{CH}_{2}\right)$, $1.51\left(\mathrm{~m}, 2 \mathrm{H},-\mathrm{CH}_{2^{-}}\right), 1.37\left(\mathrm{~m}, 2 \mathrm{H},-\mathrm{CH}_{2^{-}}\right), 1.27-1.16\left(\mathrm{~m}, 3 \mathrm{H}, \mathrm{SiCH}\left(\mathrm{CH}_{3}\right)_{2}\right), 1.16-1.08(\mathrm{~m}, 18 \mathrm{H}$, $\left.\mathrm{SiCH}\left(\mathrm{CH}_{3}\right)_{2}\right), 0.93\left(\mathrm{t}, 3 \mathrm{H}, \mathrm{J}=7.3 \mathrm{~Hz},-\mathrm{CH}_{3}\right)$.

${ }^{13} \mathbf{C}$ NMR $\left(101 \mathrm{MHz}, \mathrm{CDCl}_{3}\right) \delta 159.9,88.6,36.4,29.2,22.3,18.0,14.0,12.7$.

IR 2945 (s), 2895 (w), 2868 (s), 1674 (w), 1657 (w), 1617 (w), 1464 (m), 1272 (s), 1020 (s), 883 (s)

HRMS (ESI) calcd for $\mathrm{C}_{15} \mathrm{H}_{33} \mathrm{OSi}^{+}[\mathrm{M}+\mathrm{H}]^{+}$257.2295; found 257.2308. 


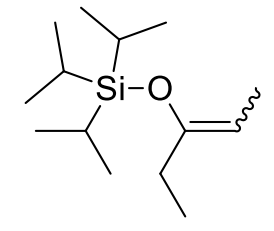

Using method A, starting from $318 \mathrm{mg}$ of 3-pentanone (3.69 mmol), $549 \mathrm{mg}$ ( $2.26 \mathrm{mmol}, 61 \%$ yield) of a colorless oil were obtained containing a 8.3:1 mixture of $\mathrm{Z} / \mathrm{E}$ isomers.

${ }^{1} \mathbf{H}$ NMR $\left(400 \mathrm{MHz}, \mathrm{CDCl}_{3}\right) \delta 4.56(\mathrm{q}, 0.12 \mathrm{H}, J=6.9 \mathrm{~Hz}, \mathrm{C}=\mathrm{CH}$ E isomer), 4.46 (qt, $\mathrm{J}=6.5,1.0 \mathrm{~Hz}$, $\mathrm{C}=\mathrm{CH} \mathrm{Z}$ isomer), 2.16-2.07 (m, 2.2 H, $\mathrm{CH}_{2} \mathrm{Z}+\mathrm{E}$ isomers), 1.64-1.59 (m, $3 \mathrm{H}, \mathrm{CHCH}_{3} \mathrm{Z}$ isomer), 1.571.54 (d, $0.4 \mathrm{H}, J=6.8 \mathrm{~Hz}, \mathrm{E}$ isomer), $1.25-1.02$ (m, $27 \mathrm{H}$, TIPS $+\mathrm{CH}_{2} \mathrm{CH}_{3} \mathrm{Z}+\mathrm{E}$ isomers).

${ }^{13} \mathbf{C ~ N M R}\left(101 \mathrm{MHz}, \mathrm{CDCl}_{3}\right.$ ) (only $\mathrm{Z}$ isomer) $\delta 153.2,99.4,29.4,18.1,13.3,11.8,10.7$.

The characterization data for $\mathbf{5 n}$ corresponded to the reported values ${ }^{13}$

\section{$\underline{\text { (Cyclohex-1-en-1-yloxy)triisopropylsilane (50): }}$}<smiles>CC(C)[Si](OC1=CCCCC1)(C(C)C)C(C)C</smiles>

Using method A, starting from $474 \mathrm{mg}$ of cyclohexanone (4.82 mmol), $1.22 \mathrm{~g}$ (4.82 mmol, $100 \%$ yield) of a colorless oil were obtained.

${ }^{1} \mathbf{H}$ NMR $\left(400 \mathrm{MHz}, \mathrm{CDCl}_{3}\right) \delta 4.88(\mathrm{~m}, 1 \mathrm{H}, \mathrm{C}=\mathrm{CH}), 2.11-1.99\left(\mathrm{~m}, 4 \mathrm{H},-\mathrm{CH}_{2^{-}}\right), 1.72-1.64(\mathrm{~m}, 2 \mathrm{H},-$ $\left.\mathrm{CH}_{2^{-}}\right), 1.57-1.50\left(\mathrm{~m}, 2 \mathrm{H},-\mathrm{CH}_{2^{-}}\right), 1.22-1.04(\mathrm{~m}, 21 \mathrm{H}, \mathrm{TIPS})$

${ }^{13} \mathbf{C}$ NMR $\left(101 \mathrm{MHz}, \mathrm{CDCl}_{3}\right) \delta 150.6,103.6,30.0,23.9,23.3,22.4,18.0,12.7$.

The characterization data for $\mathbf{5 0}$ corresponded to the reported values ${ }^{14}$

${ }^{13}$ P. Magnus, L. Barth, Tetrahedron, 1995, 51, 11075

${ }^{14}$ J. Q. Yu, H. C. Wu, E. J. Corey, Org. Lett. 2005, 7, 1415 


\section{Scope of the Reaction}

\section{Complete optimization table}

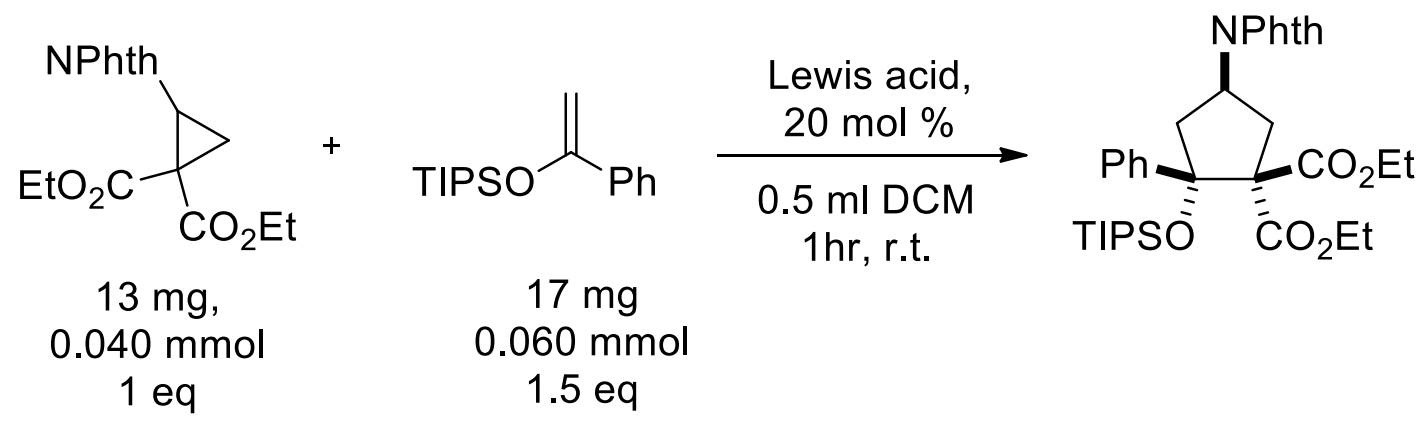

\begin{tabular}{|c|c|c|}
\hline Acid & Conversion \% & Closed/Open \\
\hline $\mathrm{SnCl}_{4}$ & $100.00^{a}$ & $>100 / 1$ \\
\hline $\mathrm{SnCl}_{4}$ & 100.00 & $1 / 1$ \\
\hline $\mathrm{Cu}(\mathrm{OTf})_{2}$ & 100.00 & $5 / 1$ \\
\hline$(\mathrm{CuOTf})_{2 . \mathrm{Tol}}$ & 100.00 & $1 / 1$ \\
\hline $\operatorname{In}(\mathrm{Otf})_{3}$ & 100.00 & $>1 / 100$ \\
\hline $\mathrm{AlCl}_{3}$ & 100.00 & $0.4 / 1$ \\
\hline $\mathrm{Sn}(\mathrm{Otf})_{2}$ & 100.00 & $2 / 1$ \\
\hline $\mathrm{Yb}(\mathrm{Otf})_{3}$ & 100.00 & $5 / 1$ \\
\hline $\mathrm{HNTf}_{2}$ & 100.00 & $>1 / 100$ \\
\hline $\mathrm{Sc}(\mathrm{Otf})_{3}$ & 100.00 & $1 / 3$ \\
\hline $\mathrm{Hf}(\mathrm{Otf})_{4}$ & 100.00 & $1 / 2$ \\
\hline $\mathrm{Zn}(\mathrm{NTf})_{2}$ & 100.00 & $1 / 2,5$ \\
\hline $\mathrm{Zn}(\mathrm{OTf})_{2}$ & 40.00 & $0.5 / 1$ \\
\hline $\mathrm{Ni}\left(\mathrm{ClO}_{4}\right)_{2}$ & 30.00 & $>10 / 1$ \\
\hline $\mathrm{CuCl}_{2}$ & 0 & nd \\
\hline AgOTf & 0 & nd \\
\hline $\mathrm{Me}_{2} \mathrm{AlCl}$ & 0 & nd \\
\hline $\mathrm{MgI}_{2}$ & 0 & nd \\
\hline $\mathrm{H}_{3} \mathrm{PO}_{4}$ & 0 & nd \\
\hline Camphorsulfonic acid & 0 & nd \\
\hline Trifluoroacetic acid & 0 & nd \\
\hline $\mathrm{BF}_{3} . \mathrm{OEt}_{2}$ & 0 & nd \\
\hline $\mathrm{Bn}_{2}$ BOTf & 0 & nd \\
\hline
\end{tabular}




\section{General procedure for the $\mathrm{SnCl}_{4}$-catalyzed synthesis of $\mathrm{N}$-phthalimide}

\section{aminocyclopentane :}
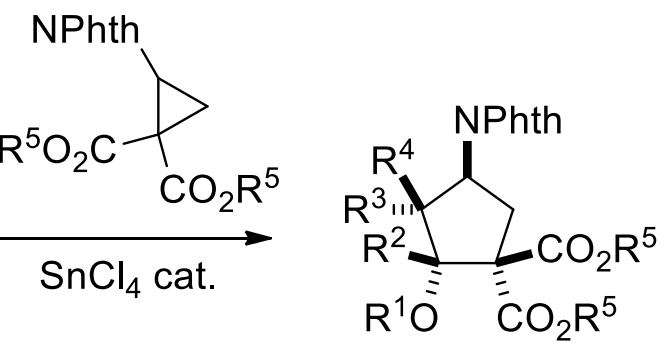

In an oven-dried flask sealed with a septum and under $\mathrm{N}_{2}$ atmosphere is added the $\mathrm{N}$-phthalimide aminocyclopropane $(0.3 \mathrm{mmol}, 1 \mathrm{eq})$ and the silyl-enol ether $(1.5 \mathrm{eq})$ in dry dichloromethane $(0.15 \mathrm{M})$. The solution is cooled down to $-78^{\circ} \mathrm{C}$ and a $0.43 \mathrm{M}$ solution of tin tetrachloride $(5 \mathrm{~mol} \%)$ in dry dichloromethane is added. The reaction is stirred for $1 \mathrm{~h}$ at $-78{ }^{\circ} \mathrm{C}$. Triethylamine $(0.2 \mathrm{~mL})$ is then added in one portion at $-78{ }^{\circ} \mathrm{C}$. The reaction is warmed at room temperature and stirred for $15 \mathrm{~min}$. Dichloromethane is removed under reduced pressure and the crude is directly purified by column chromatography (8:2 Hexane/Ethyl Acetate).

Trans-Diethyl-4-(1,3-dioxoisoindolin-2-yl)-2-phenyl-2-((triisopropylsilyl)oxy)cyclopentane-1,1dicarboxylate (6da):

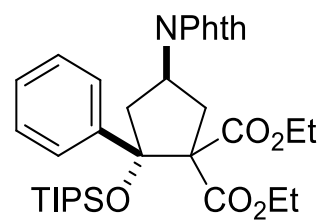

Using the method described above, $180 \mathrm{mg}(0.296 \mathrm{mmol}$, 98\%) of a single diastereomer as a colorless solid was obtained.

Rf 0.54 (7:3, Hexane/Ethyl acetate).

Mp $113.4^{\circ} \mathrm{C}$.

${ }^{1} \mathbf{H}$ NMR $\left(400 \mathrm{MHz}, \mathrm{CDCl}_{3}\right) \delta$ 7.90-7.81 (m, $4 \mathrm{H}$, Phth + Ar), $7.74(\mathrm{~m}, 2 \mathrm{H}$, Phth), 7.33-7.26 (m, $3 \mathrm{H}$, $\mathrm{Ar}$ ), $5.30(\mathrm{~m}, 1 \mathrm{H}, \mathrm{N}-\mathrm{C}-\mathrm{H}), 4.33\left(\mathrm{~m}, 2 \mathrm{H}, \mathrm{CO}_{2} \mathrm{CH}_{2}\right), 4.00-3.81$ (m, $3 \mathrm{H}, \mathrm{CO}_{2} \mathrm{CH}_{2}+\mathrm{CH}_{2}$ ), 3.42 (ddd, 1 $\left.\mathrm{H}, J=13.6,9.5,1.1 \mathrm{~Hz}, \mathrm{CH}_{2}\right), 2.89\left(\mathrm{dd}, 1 \mathrm{H}, J=13.7,8.9 \mathrm{~Hz}, \mathrm{CH}_{2}\right), 2.46(\mathrm{ddd}, 1 \mathrm{H}, \mathrm{J}=12.3,6.0,1.0$ $\left.\mathrm{Hz}, \mathrm{CH}_{2}\right), 1.36\left(\mathrm{t}, 3 \mathrm{H}, \mathrm{J}=7.1 \mathrm{~Hz}, \mathrm{CH}_{3}\right), 1.03-0.92\left(\mathrm{~m}, 24 \mathrm{H}, \mathrm{CH}_{3}+\mathrm{TIPS}\right)$

${ }^{13} \mathrm{C}$ NMR $\left(101 \mathrm{MHz}, \mathrm{CDCl}_{3}\right) \delta 170.6,168.4,168.3,142.0,134.0,132.0,128.6,127.9,127.1,123.2$, $87.5,70.1,61.5,61.0,47.9,41.8,36.3,18.2,18.2,13.7^{15}$

IR 2944 (w), 2868 (w), 2258 (w), 1775 (w), 1734 (m), 1712 (s), 1377 (s), 1253 (m), 1128 (s), 981 (m) HRMS (ESI) calcd for $\mathrm{C}_{34} \mathrm{H}_{46} \mathrm{NO}_{7} \mathrm{Si}^{+}[\mathrm{M}+\mathrm{H}]^{+} 608.3038$; found 608.3050

Recrystallized in ethanol.

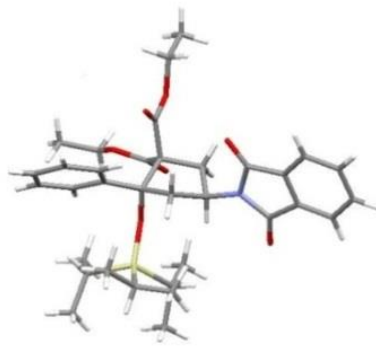

\footnotetext{
${ }^{15} \mathrm{The}^{\mathrm{CH}_{3}}$ carbons of TIPS are splitting.
} 
The crystal structure has been deposited at the Cambridge Crystallographic Data Centre and allocated the deposition number : CCDC 842232

Trans-Dimethyl-4-(1,3-dioxoisoindolin-2-yl)-2-phenyl-2-((triisopropylsilyl)oxy)cyclopentane-1,1dicarboxylate (6fa):<smiles>COC(=O)C1(C(=O)OC)CC([PbH])CC1(O)c1ccccc1</smiles>

Using the method described above, $165 \mathrm{mg}(0.284 \mathrm{mmol}, 95 \%)$ of a single diastereomer as a colorless solid was obtained.

The two enantiomers were separated by HPLC using Chiralpack IA column $(0.46 \times 25 \mathrm{~cm}), 95: 5$ Hexane/Isopropanol, $0.5 \mathrm{ml} / \mathrm{min} ; \operatorname{tr}_{1}=20.0 \mathrm{~min},[\alpha]_{\mathrm{D}}^{25.0}-40.3\left(\mathrm{c}=1, \mathrm{CHCl}_{3}\right) ; \operatorname{tr}_{2}=21.4 \mathrm{~min}$, $\mathbf{R}_{\mathbf{f}} 0.55$ (6:4, Hexane/Ethyl acetate).

Mp $134.5^{\circ} \mathrm{C}$.

${ }^{1} \mathbf{H}$ NMR $\left(400 \mathrm{MHz}, \mathrm{CDCl}_{3}\right) \delta 7.87$ (m, 2H, Phth), 7.80-7.72 (m, $4 \mathrm{H}$, Phth + Ar), 7.34-7.25 (m, $3 \mathrm{H}$, $A r), 5.29(\mathrm{~m}, 1 \mathrm{H}, \mathrm{N}-\mathrm{C}-\mathrm{H}), 3.86(\mathrm{~s}, 3 \mathrm{H}, O M e), 3.81\left(\mathrm{t}, 1 \mathrm{H}, J=12.2 \mathrm{~Hz}, C_{2}\right), 3.47-3.39(\mathrm{~m}, 4 \mathrm{H}$, $\left.\mathrm{OMe}+\mathrm{CH}_{2}\right), 2.91\left(\mathrm{dd}, 1 \mathrm{H}, J=13.7,8.7 \mathrm{~Hz}, \mathrm{CH}_{2}\right), 2.46\left(\mathrm{dd}, 1 \mathrm{H}, J=12.4,6.2 \mathrm{~Hz}, \mathrm{CH}_{2}\right), 1.01-0.92$ (m, $21 \mathrm{H}$, TIPS).

${ }^{13} \mathrm{C}$ NMR $\left(101 \mathrm{MHz}, \mathrm{CDCl}_{3}\right) \delta 171.0,168.7,168.4,141.8,134.1,132.0,128.4,128.0,127.1,123.2$, $87.6,70.1,52.4,52.1,47.8,41.7,36.2,18.2,18.2,13.7^{15}$

IR 2952 (w), 2868 (w), 2259 (w), 1738 (m), 1712 (s), 1378 (m), 1129 (s), 981 (m).

HRMS (ESI) calcd for $\mathrm{C}_{32} \mathrm{H}_{42} \mathrm{NO}_{7} \mathrm{Si}^{+}[\mathrm{M}+\mathrm{H}]^{+}$580.2725; found 580.2717 .

Trans-Dimethyl-2-((tert-butyldimethylsilyl)oxy)-4-(1,3-dioxoisoindolin-2-yl)-2phenylcyclopentane-1,1-dicarboxylate (6fb):

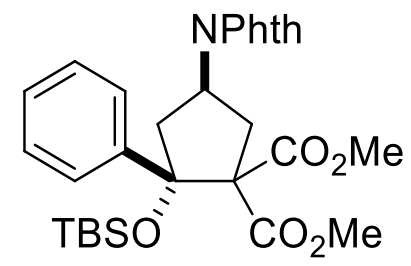

Using the method described above, $157 \mathrm{mg}(0.293 \mathrm{mmol}, 98 \%)$ of a single diastereomer as a colorless solid was obtained.

$\mathbf{R}_{\mathbf{f}} 0.38$ (7:3, Hexane/Ethyl acetate).

Mp $162.9^{\circ} \mathrm{C}$.

${ }^{1} \mathbf{H}$ NMR $\left(400 \mathrm{MHz}, \mathrm{CDCl}_{3}\right) \delta 7.86(\mathrm{~m}, 2 \mathrm{H}$, Phth), 7.79-7.68 (m, $4 \mathrm{H}$, Phth + Ar), 7.36-7.26 (m, $3 \mathrm{H}$, $A r), 5.27$ (m, $1 \mathrm{H}, N-C-H), 3.87$ (s, $3 \mathrm{H}, O M e), 3.78\left(\mathrm{dd}, 1 \mathrm{H}, J=12.2,12.2 \mathrm{~Hz}, \mathrm{CH}_{2}\right), 3.47(\mathrm{~s}, 3 \mathrm{H}$, $O M e), 3.41\left(\mathrm{ddd}, 1 \mathrm{H}, J=13.5,9.3,1.2 \mathrm{~Hz}, \mathrm{CH}_{2}\right), 2.93\left(\mathrm{dd}, 1 \mathrm{H}, J=13.6,8.9 \mathrm{~Hz}, \mathrm{CH}_{2}\right), 2.36(\mathrm{dd}, 1 \mathrm{H}$, $\left.\mathrm{J}=12.0,5.0 \mathrm{~Hz}, C_{2}\right), 0.95(\mathrm{~s}, 9 \mathrm{H}, T B S), 0.04(\mathrm{~s}, 3 \mathrm{H}, T B S),-0.47(\mathrm{~s}, 3 \mathrm{H}, T B S)$.

${ }^{13}$ C NMR $\left(101 \mathrm{MHz}, \mathrm{CDCl}_{3}\right) \delta 170.9,168.7,168.3,141.3,134.1,132.0,128.4,127.9,127.2,123.2$, $87.1,69.8,52.4,52.1,47.7,41.7,36.2,25.8,18.5,-2.5,-3.5$. 
IR 2953 (w), 2932 (w), 2887 (w), 2857 (w), 2258 (w), 1737 (m), 1712 (s), 1378 (m), 1128 (m), 909 (s).

HRMS (ESI) calcd for $\mathrm{C}_{29} \mathrm{H}_{36} \mathrm{NO}_{7} \mathrm{Si}^{+}[\mathrm{M}+\mathrm{Na}]^{+}$560.2075; found 560.2012.

Trans-Dimethyl-4-(1,3-dioxoisoindolin-2-yl)-2-phenyl-2-((trimethylsilyl)oxy)cyclopentane-1,1dicarboxylate (6fc):

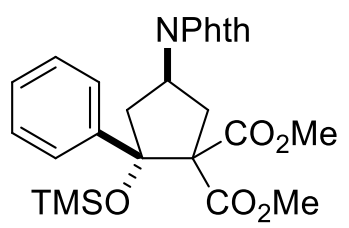

Using the method described above, $141 \mathrm{mg}(0.285 \mathrm{mmol}$, 95\%) of a single diastereomer as a colorless solid was obtained.

$\mathbf{R}_{\mathbf{f}} 0.35$ (7:3, Hexane/Ethyl acetate).

Mp $139.5^{\circ} \mathrm{C}$.

${ }^{1} \mathbf{H}$ NMR $\left(400 \mathrm{MHz}, \mathrm{CDCl}_{3}\right) \delta 7.87$ (m, $2 \mathrm{H}$, Phth), 7.76 (m, 2 H, Phth), 7.69-7.65 (m, $2 \mathrm{H}$, Ar), 7.35$7.27(\mathrm{~m}, 3 \mathrm{H}, A r), 5.23(\mathrm{~m}, 1 \mathrm{H}, N-C-H), 3.85(\mathrm{~s}, 3 \mathrm{H}, O M e), 3.80$ (t, $\left.1 \mathrm{H}, J=12.2 \mathrm{~Hz}, C_{2}\right), 3.49$ (s, 3 $\mathrm{H}, \mathrm{OMe}$ ), 3.36 (ddd, $1 \mathrm{H}, J=13.5,9.3,1.4 \mathrm{~Hz}, \mathrm{CH}_{2}$ ), 2.93 (dd, $1 \mathrm{H}, J=13.6,9.0 \mathrm{~Hz}, \mathrm{CH}_{2}$ ), 2.28 (ddd, $\left.1 \mathrm{H}, J=12.2,5.9,1.2 \mathrm{~Hz}, \mathrm{CH}_{2}\right),-0.03$ (s, $\left.9 \mathrm{H}, T M S\right)$.

${ }^{13} \mathrm{C}$ NMR $\left(101 \mathrm{MHz}, \mathrm{CDCl}_{3}\right) \delta 170.7,168.9,168.4,141.3,134.1,132.0,128.2,127.8,127.2,123.2$, 87.2, 69.8, 52.2, 52.1, 47.9, 41.6, 35.8, 1.6.

IR 2953 (w), 2924 (w), 2850 (w), 1773 (w), 1738 (s), 1712 (s), 1379 (m), 1253 (m), 1131 (m), 985 (m), 845 (s).

HRMS (ESI) calcd for $\mathrm{C}_{26} \mathrm{H}_{30} \mathrm{NO}_{7} \mathrm{Si}^{+}[\mathrm{M}+\mathrm{H}]+496.1786$; found 496.1800

Trans-Dimethyl-4-(1,3-dioxoisoindolin-2-yl)-2-(4-fluorophenyl)-2-((triisopropylsilyl)oxy)cyclopentane-1,1-dicarboxylate (6fd):

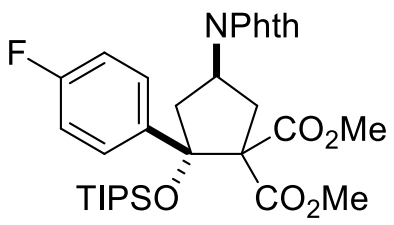

Using the method described above, $163 \mathrm{mg}(0.273 \mathrm{mmol}, 91 \%)$ of a single diastereomer as a colorless solid was obtained.

$\mathbf{R}_{\mathbf{f}} 0.6$ (6:4, Hexane/Ethyl acetate).

Mp $152.5^{\circ} \mathrm{C}$.

${ }^{1} \mathbf{H}$ NMR $\left(400 \mathrm{MHz}, \mathrm{CDCl}_{3}\right) \delta 7.86(\mathrm{~m}, 2 \mathrm{H}$, Phth), 7.79-7.73 (m, $4 \mathrm{H}$, Phth + Ar), $7.00(\mathrm{t}, 2 \mathrm{H}, J=$ $8.7 \mathrm{~Hz}, A r), 5.27(\mathrm{~m}, 1 \mathrm{H}, \mathrm{N}-\mathrm{C}-\mathrm{H}), 3.86(\mathrm{~s}, 3 \mathrm{H}, \mathrm{OMe}), 3.79\left(\mathrm{dd}, 1 \mathrm{H}, J=12.2,12.2 \mathrm{~Hz}, C_{2}\right), 3.51$ (s, $3 \mathrm{H}, \mathrm{OMe}$ ), 3.44 (ddd, $1 \mathrm{H}, J=13.8,9.7,0.9 \mathrm{~Hz}, \mathrm{CH}_{2}$ ), 2.89 (dd, $1 \mathrm{H}, J=13.9,8.4 \mathrm{~Hz}$ ), $\mathrm{CH}_{2}, 2.44$ (dd, $\left.1 \mathrm{H}, J=12.5,6.3 \mathrm{~Hz}, \mathrm{CH}_{2}\right), 1.03-0.93(\mathrm{~m}, 21 \mathrm{H}$, TIPS).

${ }^{13} \mathrm{C} \mathrm{NMR}\left(101 \mathrm{MHz}, \mathrm{CDCl}_{3}\right) \delta 170.9,168.6,168.3,162.4(\mathrm{~d}, J=247 \mathrm{~Hz}), 137.7$ (d, $\left.J=4 \mathrm{~Hz}\right), 134.1$, 132.0, $130.4(\mathrm{~d}, J=8 \mathrm{~Hz}), 123.2,113.9(\mathrm{~d}, J=21 \mathrm{~Hz}), 87.1,70.0,52.4,52.3,47.6,41.9,36.1,18.2$, $18.1,13.7^{15}$

IR 2951 (w), 2894 (w), 2869 (w), 1775 (w), 1739 (m), 1714 (s), 1514 (w), 1379 (m), 1129 (m)

HRMS (ESI) calcd for $\mathrm{C}_{32} \mathrm{H}_{41} \mathrm{FNO}_{7} \mathrm{Si}[\mathrm{M}+\mathrm{H}]^{+}$598.2631; found 598.2708. 
Trans-Dimethyl-4-(1,3-dioxoisoindolin-2-yl)-2-(4-(methoxycarbonyl)phenyl)-2-((triisopropylsilyl)oxy) cyclopentane-1,1-dicarboxylate (6fe):

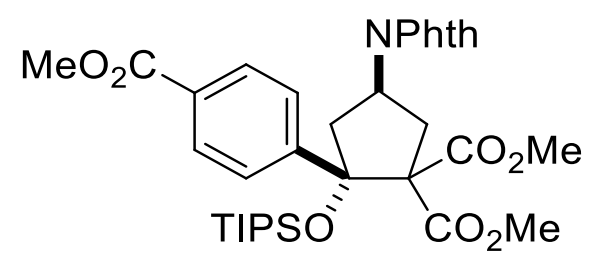

Using the method described above, $181 \mathrm{mg}(0.284 \mathrm{mmol}, 95 \%)$ of a single diastereomer as a colorless solid was obtained.

$\mathbf{R}_{\mathbf{f}} 0.5$ (6:4, Hexane/Ethyl acetate).

Mp $151.5^{\circ} \mathrm{C}$.

${ }^{1} \mathbf{H}$ NMR $\left(400 \mathrm{MHz}, \mathrm{CDCl}_{3}\right) \delta 7.99$ (m, $\left.2 \mathrm{H}, A r\right), 7.88-7.82(\mathrm{~m}, 4 \mathrm{H}, A r+$ Phth), 7.75 (m, $2 \mathrm{H}$, Phth), $5.28(\mathrm{~m}, 1 \mathrm{H}, \mathrm{N}-\mathrm{C}-\mathrm{H}), 3.92(\mathrm{~s}, 3 \mathrm{H}, \mathrm{OMe}), 3.85(\mathrm{~s}, 3 \mathrm{H}, \mathrm{OMe}), 3.81\left(\mathrm{dd}, 1 \mathrm{H}, \mathrm{J}=12.4 \mathrm{~Hz}, \mathrm{CH}_{2}\right), 3.52-$ $3.42\left(\mathrm{~m}, 4 \mathrm{H}, \mathrm{OMe}+\mathrm{CH}_{2}\right), 2.88\left(\mathrm{dd}, 1 \mathrm{H}, J=13.9,8.1 \mathrm{~Hz}, \mathrm{CH}_{2}\right), 2.47(\mathrm{dd}, 1 \mathrm{H}, J=12.4,6.2 \mathrm{~Hz}$, $\left.\mathrm{CH}_{2}\right), 1.03-0.91(\mathrm{~m}, 21 \mathrm{H}, \mathrm{TIPS})$.

${ }^{13}$ C NMR $\left(101 \mathrm{MHz}, \mathrm{CDCl}_{3}\right) \delta 170.7,168.5,168.3,166.9,146.7,134.1,131.9,129.5,128.7,128.3$, $123.3,87.2,70.0,52.5,52.3,52.1,47.4,41.7,36.1,18.2,18.1,13.7^{15}$

IR 2953 (w), 2870 (w), 2256 (w), 1712 (m), 1283 (w), 908 (s), 731 (s)

HRMS (ESI) calcd for $\mathrm{C}_{34} \mathrm{H}_{43} \mathrm{NNaO}_{9} \mathrm{Si}^{+}[\mathrm{M}+\mathrm{Na}]^{+} 660.2599$; found 660.2604

Trans-Dimethyl-2-(4-cyanophenyl)-4-(1,3-dioxoisoindolin-2-yl)-2-((triisopropylsilyl)oxy)cyclopentane-1,1-dicarboxylate (6ff):

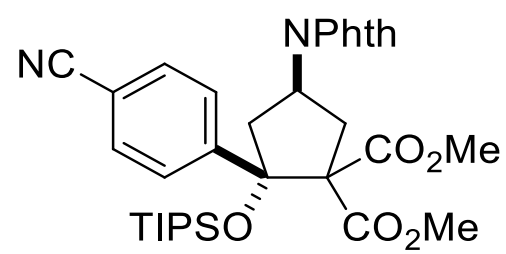

Using the method described above, $127 \mathrm{mg}(0.210 \mathrm{mmol}, 70 \%)$ of a single diastereomer as a colorless solid was obtained.

$\mathbf{R}_{\mathbf{f}} 0.42$ (6:4, Hexane/Ethyl acetate).

Mp $116.5^{\circ} \mathrm{C}$.

${ }^{1} \mathbf{H}$ NMR $\left(400 \mathrm{MHz}, \mathrm{CDCl}_{3}\right) \delta$ 7.93-7.84 (m, $\left.4 \mathrm{H}, A r+P h t h\right), 7.76(\mathrm{~m}, 2 \mathrm{H}$, Phth), $7.63(\mathrm{~m}, 2 \mathrm{H}, A r)$, $5.27(\mathrm{~m}, 1 \mathrm{H}, \mathrm{N}-\mathrm{C}-\mathrm{H}), 3.85$ (s, $3 \mathrm{H}, \mathrm{OMe}$ ), $3.77\left(\mathrm{dd}, 1 \mathrm{H}, 12.2,12.2 \mathrm{~Hz}, \mathrm{CH}_{2}\right), 3.55$ (s, $3 \mathrm{H}, \mathrm{OMe}$ ), 3.50 (dd, $1 \mathrm{H}, J=14.1,10.2 \mathrm{~Hz}, \mathrm{CH}_{2}$ ), 2.86 (dd, $1 \mathrm{H}, J=14.1,7.8 \mathrm{~Hz}, \mathrm{CH}_{2}$ ), 2.46 (dd, $1 \mathrm{H}, J=12.5,6.5$ $\left.\mathrm{Hz}, \mathrm{CH}_{2}\right), 1.04-0.91$ (m, $21 \mathrm{H}$, TIPS).

${ }^{13}$ C NMR $\left(101 \mathrm{MHz}, \mathrm{CDCl}_{3}\right) \delta 170.6,168.3,168.2,146.9,134.2,131.9,130.8,129.5,123.3,118.9$, $111.8,86.9,69.9,52.6,52.5,47.0,41.5,36.0,18.2,18.1,13.8^{15}$

IR 2951 (w), 2869 (w), 2230 (w), 1739 (m), 1712 (s), 1380 (m), 1136 (m), 1124 (m), 983 (m), 912 (m), 735 (s), 727 (s)

HRMS (ESI) calcd for $\mathrm{C}_{33} \mathrm{H}_{40} \mathrm{~N}_{2} \mathrm{NaO}_{7} \mathrm{Si}^{+}[\mathrm{M}+\mathrm{Na}]^{+} 627.2497$; found 627.2499 
Trans-Dimethyl 4-(1,3-dioxoisoindolin-2-yl)-2-(4-methoxyphenyl)-2-((triisopropylsilyl)oxy)cyclopentane-1,1-dicarboxylate (6fg):<smiles>COC(=O)C1(C(=O)OC)C[C@@H](P)CC1(O)c1ccc(OC)cc1</smiles>

Using the method described above, $182 \mathrm{mg}(0.298 \mathrm{mmol}$, 99\%) of a single diastereomer as a colorless solid was obtained.

$\mathbf{R}_{\mathbf{f}} 0.48$ (6:4, Hexane/Ethyl acetate).

Mp $142.3^{\circ} \mathrm{C}$.

${ }^{1} \mathbf{H}$ NMR $\left(400 \mathrm{MHz}, \mathrm{CDCl}_{3}\right) \delta 7.86(\mathrm{~m}, 2 \mathrm{H}, P h t h), 7.74(\mathrm{~m}, 2 \mathrm{H}, P h t h), 7.69(\mathrm{~d}, \mathrm{~J}=9.0 \mathrm{~Hz}, 2 \mathrm{H}, A r)$, $6.83(\mathrm{~d}, \mathrm{~J}=9.0 \mathrm{~Hz}, 2 \mathrm{H}, A r), 5.27(\mathrm{~m}, 1 \mathrm{H}, N-C-H), 3.86$ (s, $3 \mathrm{H}, O M e), 3.83$ (s, $3 \mathrm{H}, O M e$ ), 3.78 (dd, $\left.1 \mathrm{H}, J=12.2,12.2 \mathrm{~Hz}, \mathrm{CH}_{2}\right), 3.51\left(\mathrm{~s}, 3 \mathrm{H}, \mathrm{OMe}\right.$ ), $3.42\left(\mathrm{ddd}, 1 \mathrm{H}, \mathrm{J}=13.6,9.7,0.7 \mathrm{~Hz}, \mathrm{CH}_{2}\right), 2.89$ (dd, $\left.1 \mathrm{H}, J=13.8,8.6 \mathrm{~Hz}, \mathrm{CH}_{2}\right), 2.43\left(\mathrm{dd}, 1 \mathrm{H}, J=12.3,6.2 \mathrm{~Hz}, \mathrm{CH}_{2}\right), 1.04-0.93$ (m, $21 \mathrm{H}$, TIPS)

${ }^{13} \mathrm{C}$ NMR $\left(101 \mathrm{MHz}, \mathrm{CDCl}_{3}\right) \delta 171.1,168.8,168.3,159.1,134.0,133.99,132.0,129.7,123.2,112.3$, $87.4,70.0,55.2,52.3,52.2,47.8,41.9,36.2,18.2,18.2,13.7^{15}$

IR 2949 (w), 2868 (w), 1776 (w), 1737 (m), 1712 (s), 1378 (m), 1255 (m), 1125 (m), 981 (m), 722 (s). HRMS (ESI) calcd for $\mathrm{C}_{33} \mathrm{H}_{43} \mathrm{NNaO}_{8} \mathrm{Si}^{+}[\mathrm{M}+\mathrm{Na}]^{+}$632.2650; found 632.2651.

Trans-Dimethyl-2-(2-bromophenyl)-4-(1,3-dioxoisoindolin-2-yl)-2-((triisopropylsilyl)oxy)cyclopentane-1,1-dicarboxylate (6fh):

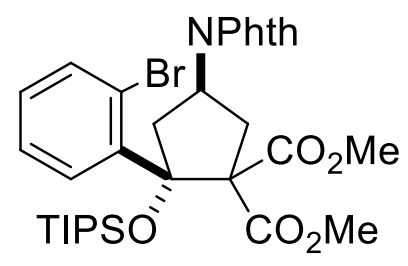

Using the method described above, $183 \mathrm{mg}(0.277 \mathrm{mmol}, 92 \%)$ of a single diastereomer as a colorless solid was obtained.

The two enantiomers were separated by HPLC using Chiralpack IC column $(0.46 \times 25 \mathrm{~cm})$, 95:5 Hexane/Isopropanol, $1 \mathrm{ml} / \mathrm{min} ; \operatorname{tr}_{1}=20.9 \mathrm{~min} ; \operatorname{tr}_{2}=24.9 \mathrm{~min},[\alpha]_{\mathrm{D}}{ }^{25.0}-43.0\left(\mathrm{c}=1, \mathrm{CHCl}_{3}\right)$

$\mathbf{R}_{\mathbf{f}} 0.46$ (6:4, Hexane/Ethyl acetate).

Mp $155.7^{\circ} \mathrm{C}$.

${ }^{1} \mathbf{H}$ NMR $\left(400 \mathrm{MHz}, \mathrm{CDCl}_{3}\right) \delta$ 7.88-7.81 (m, $3 \mathrm{H}$, Phth +Ar), $7.71(\mathrm{~m}, 2 \mathrm{H}$, Phth), $7.57(\mathrm{dd}, 1 \mathrm{H}, J=$ 8.0, $1.4 \mathrm{~Hz}, A r), 7.24(\mathrm{~m}, 1 \mathrm{H}, A r), 7.06(\mathrm{~m}, 1 \mathrm{H}, A r), 5.22(\mathrm{~m}, 1 \mathrm{H}, N-C-H), 4.40(\mathrm{dd}, 1 \mathrm{H}, \mathrm{J}=12.8$, $12.8 \mathrm{~Hz}, \mathrm{CH}_{2}$ ), 3.79 (s, $3 \mathrm{H}, \mathrm{OMe}$ ), 3.60 (s, $3 \mathrm{H}, \mathrm{OMe}$ ), 3.35 (dd, $1 \mathrm{H}, \mathrm{J}=13.5,10.0 \mathrm{~Hz}, \mathrm{CH}_{2}$ ), 2.78 (dd, $\left.1 \mathrm{H}, J=13.7,7.4 \mathrm{~Hz}, C_{2}\right), 2.60\left(\mathrm{dd}, 1 \mathrm{H}, J=12.9,6.0 \mathrm{~Hz}, C_{2}\right), 1.04-0.94(\mathrm{~m}, 21 \mathrm{H}, T I P S)$.

${ }^{13}$ C NMR $\left(101 \mathrm{MHz}, \mathrm{CDCl}_{3}\right) \delta 174.4,172.0,171.6,143.1,137.1,135.8,135.2,133.7,130.8,127.3$, $124.6,123.2,89.0,70.3,50.4,50.4,45.7,40.8,33.5,14.9,14.7,10.3 .{ }^{15}$

IR $2949(\mathrm{w}), 2868(\mathrm{w}), 2259$ (w), 2255 (w), $1774(\mathrm{w}), 1758$ (m), 1737 (s), 1712 (s), 1378 (m), 913 (m), $734(\mathrm{~s}), 722(\mathrm{~s})$

HRMS (ESI) calcd for $\mathrm{C}_{32}{ }^{79} \mathrm{BrH}_{41} \mathrm{NO}_{7} \mathrm{Si}^{+}[\mathrm{M}+\mathrm{H}]^{+} 658.1830$; found 658.1835 
Trans- Dimethyl 4-(1,3-dioxoisoindolin-2-yl)-2-(naphthalen-2-yl)-2-((triisopropylsilyl)oxy)cyclopentane-1,1-dicarboxylate (6fi):

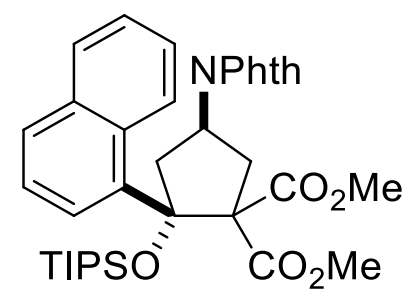

Using the method described above, $171 \mathrm{mg}(0.272 \mathrm{mmol}, 91 \%)$ of a single diastereomer as a colorless solid were obtained.

$\mathbf{R}_{\mathbf{f}} 0.6$ (6:4, Hexane/Ethyl acetate)

Mp $193.8^{\circ} \mathrm{C}$.

${ }^{1} \mathbf{H}$ NMR $\left(400 \mathrm{MHz}, \mathrm{CDCl}_{3}\right) \delta 8.39(\mathrm{~d}, 1 \mathrm{H}, J=8.4 \mathrm{~Hz}, A r), 7.90-7.79(\mathrm{~m}, 5 \mathrm{H}$, Phth + Ar) 7.75 (m, 2 H, Phth), 7.45 (t, $1 \mathrm{H}, J=7.8 \mathrm{~Hz}, A r), 7.42-7.33$ (m, $2 \mathrm{H}, A r), 5.42(\mathrm{~m}, 1 \mathrm{H}, N-C-H), 3.90$ (dd, $1 \mathrm{H}, J=$ $\left.12.5,1.5 \mathrm{~Hz}, \mathrm{CH}_{2}\right), 3.76$ (dd, $1 \mathrm{H}, J=14.3,11.7 \mathrm{~Hz}, C_{2}$ ), 3.63 (s, $3 \mathrm{H}, \mathrm{OMe}$ ), 3.43 (s, $3 \mathrm{H}, \mathrm{OMe}$ ), $2.95\left(\mathrm{dd}, 1 \mathrm{H}, J=14.4,4.7 \mathrm{~Hz}, C_{2}\right), 2.67\left(\mathrm{dd}, 1 \mathrm{H}, J=12.5,7.3 \mathrm{~Hz}, C_{2}\right), 1.08-1.03(\mathrm{~m}, 12 \mathrm{H}$, TIPS), 0.73-0.68 (m, $9 \mathrm{H}$, TIPS)

${ }^{13}$ C NMR $\left(101 \mathrm{MHz}, \mathrm{CDCl}_{3}\right) \delta 171.0,169.8,168.3,136.4,134.5,134.0,132.4,132.0,129.7,128.8$, $128.5,124.7,124.0,123.6,123.3,90.9,68.6,52.4,52.2,45.5,43.7,37.8,18.5,17.9,14.3^{15}$

IR 3050 (w), 2949 (w), 2894 (w), 2868 (w), 2258 (w), 1775 (w), 1762 (w), 1734 (s), 1712 (s), 1378 (m), $1123(\mathrm{~m}), 970(\mathrm{~m}), 910(\mathrm{~m}), 732(\mathrm{~s})$.

HRMS (ESI) calcd for $\mathrm{C}_{36} \mathrm{H}_{44} \mathrm{NO}_{7} \mathrm{Si}^{+}[\mathrm{M}+\mathrm{H}]^{+}$630.2882; found 630.2876.

Dimethyl 4-(1,3-dioxoisoindolin-2-yl)-3-methyl-2-phenyl-2-((triisopropylsilyl)oxy)cyclo-pentane1,1-dicarboxylate (6fj):

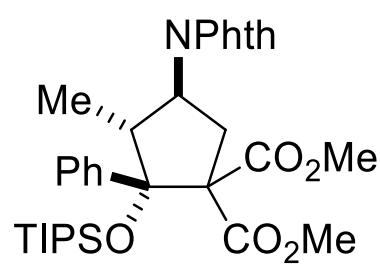

Using the method described above, $164 \mathrm{mg}(0.275 \mathrm{mmol}, 92 \%)$ of two diastereomers (20:1) as a colorless solid was obtained.

The two enantiomers were separated by HPLC using Chiralpack IA column $(0.46 \times 25 \mathrm{~cm}), 93: 7$ Hexane/Isopropanol, $1 \mathrm{ml} / \mathrm{min} ; \operatorname{tr}_{1}=7.5 \mathrm{~min},[\alpha]_{\mathrm{D}}{ }^{25.0} 48.4\left(\mathrm{c}=1, \mathrm{CHCl}_{3}\right) ; \operatorname{tr}_{2}=12.8 \mathrm{~min}$.

Major diastereomer

$\mathbf{R}_{\mathbf{f}} 0.52$ (6:4, Hexane/Ethyl acetate).

Mp $120^{\circ} \mathrm{C}$.

${ }^{1} \mathbf{H}$ NMR $\left(400 \mathrm{MHz}, \mathrm{CDCl}_{3}\right) \delta 7.84$ (m, $2 \mathrm{H}$, Phth), 7.73 (m, 2 H, Phth), 7.69-7.65 (m, $2 \mathrm{H}$, Ar), 7.36$7.27(\mathrm{~m}, 3 \mathrm{H}, \mathrm{Ar}), 4.90(\mathrm{td}, 1 \mathrm{H}, J=11.1,6.9 \mathrm{~Hz}, \mathrm{~N}-\mathrm{C}-\mathrm{H}), 3.99\left(\mathrm{~m}, 1 \mathrm{H}, \mathrm{CHCH}_{3}\right), 3.78(\mathrm{~s}, 3 \mathrm{H}, \mathrm{OMe})$, $3.53\left(\mathrm{~s}, 3 \mathrm{H}, \mathrm{OMe}\right.$ ), 3.45 (dd, $\left.1 \mathrm{H}, \mathrm{J}=14.3,11.0 \mathrm{~Hz}, \mathrm{CH}_{2}\right), 2.72\left(\mathrm{dd}, \mathrm{J}=14.3,6.9 \mathrm{~Hz}, 1 \mathrm{H}, \mathrm{CH}_{2}\right), 1.25-$ $1.07(\mathrm{~m}, 15 \mathrm{H}$, TIPS + Me), 0.99-0.95 (m, $9 \mathrm{H}$, TIPS).

${ }^{13} \mathrm{C}$ NMR $\left(101 \mathrm{MHz}, \mathrm{CDCl}_{3}\right) \delta 170.8,168.7,168.5,139.6,134.0,131.9,129.6,127.8,126.7,123.2$, $90.6,70.1,53.1,52.3,43.4,34.1,18.8,18.7,15.1,11.9^{15}$ 
IR 2950 (w), 2869 (w), 1758 (w), 1739 (s), 1739 (s), 1717 (s), 1381 (m), 1126 (m), 735 (m), 724 (s). HRMS (ESI) calcd for $\mathrm{C}_{33} \mathrm{H}_{43} \mathrm{NNaO}_{7} \mathrm{Si}^{+}[\mathrm{M}+\mathrm{Na}]^{+}$616.2701; found 616.2717.

Dimethyl-3-(1,3-dioxoisoindolin-2-yl)-9b-((triisopropylsilyl)oxy)-3,3a,4,5-tetrahydro-1Hcyclopenta[a]naphthalene-1,1(2H,9bH)-dicarboxylate (6fk):<smiles>COC(=O)C1(C(C)=O)C[C@H](c2ccccc2)C[C@H]1c1ccccc1</smiles>

Using the method described above, $166 \mathrm{mg}(0.274 \mathrm{mmol}, 91 \%)$ of two diastereomers (10:1) as a colorless solid were obtained.

The major diastereomer was recrystallized from isopropanol:

$\mathbf{R}_{\mathbf{f}} 0.50$ (6:4, Hexane/Ethyl acetate).

Mp $179.1^{\circ} \mathrm{C}$

${ }^{1} \mathbf{H}$ NMR $\left(400 \mathrm{MHz}, \mathrm{CDCl}_{3}\right) \delta 8.20(\mathrm{~d}, 1 \mathrm{H}, \mathrm{J}=7.0 \mathrm{~Hz}, A r), 7.88(\mathrm{~m}, 2 \mathrm{H}$, Phth), $7.76(\mathrm{~m}, 2 \mathrm{H}$, Phth), $7.22(\mathrm{dd}, 1 \mathrm{H}, \mathrm{J}=7.2,7.2 \mathrm{~Hz}, A r), 7.16(\mathrm{dd}, 1 \mathrm{H}, J=7.4,7.0 \mathrm{~Hz}, A r), 7.07(\mathrm{~d}, 1 \mathrm{H}, \mathrm{J}=7.6 \mathrm{~Hz}, A r)$, $5.17(\mathrm{~m}, 1 \mathrm{H}, \mathrm{N}-\mathrm{C}-\mathrm{H}), 3.98\left(\mathrm{dd}, 1 \mathrm{H}, J=12.9,12.9 \mathrm{~Hz}, \mathrm{CH}_{2}-\mathrm{C}\left(\mathrm{CO}_{2} \mathrm{Me}\right)_{2}\right), 3.88$ (s, $\left.3 \mathrm{H}, \mathrm{OMe}\right), 3.32$ (m, $1 \mathrm{H},-\mathrm{CH}-), 3.26(\mathrm{~s}, 3 \mathrm{H}, \mathrm{OMe}), 3.04\left(\mathrm{~m}, 1 \mathrm{H}, \mathrm{Ar}-\mathrm{CH}_{2}\right), 2.63\left(\mathrm{~m}, 1 \mathrm{H}, \mathrm{Ar}-\mathrm{CH}_{2}\right), 2.16(\mathrm{dd}, 1 \mathrm{H}, J=$ 12.6, $\left.5.6 \mathrm{~Hz}, \mathrm{CH}_{2}-\mathrm{C}\left(\mathrm{CO}_{2} \mathrm{Me}\right)_{2}\right), 1.87\left(\mathrm{~m}, 1 \mathrm{H}, \mathrm{Ar}-\mathrm{CH}_{2}-\mathrm{CH}_{2}\right), 1.66\left(\mathrm{~m}, 1 \mathrm{H}, \mathrm{Ar}-\mathrm{CH}_{2}-\mathrm{CH}_{2}\right), 1.10-0.87$ (m, $21 \mathrm{H}$, TIPS)

${ }^{13} \mathrm{C}$ NMR $\left(101 \mathrm{MHz}, \mathrm{CDCl}_{3}\right) \delta 170.8,168.5,168.3,140.2,138.2,134.1,131.9,129.2,127.5,127.3$, $126.1,123.3,83.4,71.0,52.5,52.1,50.3,49.8,34.3,25.3,21.2,18.4,18.0,14.0^{15}$

IR 2950 (w), 2868 (w), 2256 (w), 1773 (w), 1715 (s), 1381 (m), 1128 (m), 908 (s), 731 (s)

HRMS (ESI) calcd for $\mathrm{C}_{34} \mathrm{H}_{43} \mathrm{NNaO}_{7} \mathrm{Si}^{+}[\mathrm{M}+\mathrm{Na}]^{+}$628.2701; found 628.2722.

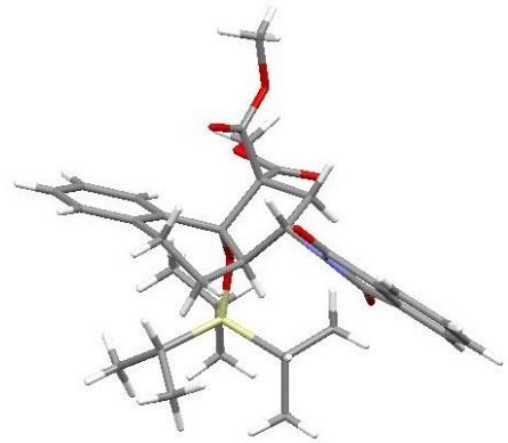

The crystal structure has been deposited at the Cambridge Crystallographic Data Centre and allocated the deposition number : CCDC 842233 
Dimethyl-4-(1,3-dioxoisoindolin-2-yl)-3,3-dimethyl-2-phenyl-2-((triisopropylsilyl)oxy)cyclopen$\underline{\text { tane-1,1-dicarboxylate (6fl): }}$

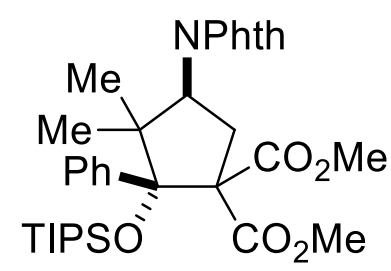

Isomer 1

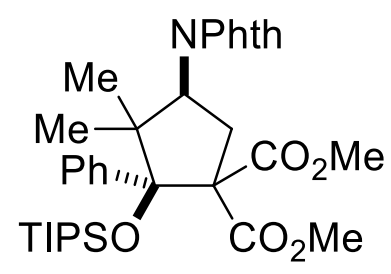

Isomer 2

Using the method described above, $147 \mathrm{mg}(0.242 \mathrm{mmol}, 81 \%)$ of two non-separable diastereomers $(4: 1)$ as a colorless solid were obtained.

$\mathbf{R}_{\mathbf{f}} 0.52$ (6:4, Hexane/Ethyl acetate)

${ }^{1} \mathbf{H}$ NMR $\left(400 \mathrm{MHz}, \mathrm{CDCl}_{3}\right) \delta 7.93(\mathrm{~m}, 2 \mathrm{H}$, Ar, Isomer $1 \&$ 2), $7.84(\mathrm{~m}, 2.5 \mathrm{H}$, Phth, Isomer 1 \& 2), 7.79-7.72 (m, $3 \mathrm{H}$, Phth + Ar, Isomer 1 \& 2), $7.51(\mathrm{~m}, 0.5 \mathrm{H}$, Ar, Isomer 2), 7.34-7.24 (m, $4 \mathrm{H}, A r$, Isomer 1 \& 2), $5.12(\mathrm{dd}, 1 \mathrm{H}, J=13.0,7.3 \mathrm{~Hz}, \mathrm{~N}-\mathrm{C}$-H, Isomer 1$), 4.77(\mathrm{~m}, 0.25 \mathrm{H}, \mathrm{N}-\mathrm{C}-\mathrm{H}$, Isomer 2), 4.48 (dd, $0.25 \mathrm{H}, \mathrm{J}=13.4,13.4 \mathrm{~Hz}, \mathrm{CH}_{2}$, Isomer 2), 3.86 (s, $3 \mathrm{H}$, OMe, Isomer 1), 3.78 (s, $0.75 \mathrm{H}$, OMe, Isomer 2), 3.69-3.61 (m, 1.75 H, OMe, Isomer 2, $\mathrm{CH}_{2}$, Isomer 1), 3.55 (s, $3 \mathrm{H}$, OMe, Isomer 1), $3.02\left(\mathrm{dd}, 1 \mathrm{H}, \mathrm{J}=13.4,7.4 \mathrm{~Hz}, \mathrm{CH}_{2}\right.$, Isomer 1), $2.40\left(\mathrm{dd}, 0.25 \mathrm{H}, \mathrm{J}=13.3,7.4 \mathrm{~Hz}, \mathrm{CH}_{2}\right.$, Isomer 2), 1.52 (s, $3 \mathrm{H}$, Me, Isomer 1), 1.41 (s, $0.75 \mathrm{H}$, Me, Isomer 2), 1.31 (s, $3 \mathrm{H}$, Me, Isomer 1), 1.04 (s, 0.75 $\mathrm{H}$, Me, Isomer 2), 1.02-0.98 (m, $12 \mathrm{H}$, TIPS, Isomer 1 \& 2), 0.97-0.92 (m, $10 \mathrm{H}$, TIPS, Isomer 1 \& 2), 0.90-0.80 (m, 4 H, TIPS, Isomer 1 \& 2)

${ }^{13} \mathrm{C}$ NMR $\left(101 \mathrm{MHz}, \mathrm{CDCl}_{3}\right) \delta 172.2,170.2,169.7,169.3,141.8,141.4,134.1,134.0,131.8,129.5$, $128.5,127.7,127.4,127.2,126.9,123.2,93.3,69.6,58.8,57.8,53.3,52.9,52.7,52.5,52.4,32.9,32.5$, $29.0,25.2,23.6,21.4,19.0,18.8,18.8,15.0,14.6^{15,16}$

IR 2949 (w), 2869 (w), 1777 (w), 1735 (m), 1716 (s), 1373 (m), 1034 (m), 911 (m), 731 (s).

HRMS (ESI) calcd for $\mathrm{C}_{34} \mathrm{H}_{46} \mathrm{NO}_{7} \mathrm{Si}^{+}[\mathrm{M}+\mathrm{H}]^{+}$608.3038; found 608.3010

Trans-dimethyl 2-butyl-4-(1,3-dioxoisoindolin-2-yl)-2-((triisopropylsilyl)oxy)cyclopentane-1,1dicarboxylate (6fm):

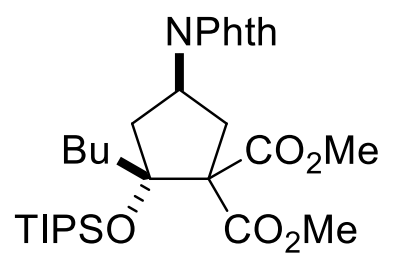

Using the method described above, $164 \mathrm{mg}(0.293 \mathrm{mmol}, 98 \%)$ of a colorless oil were obtained.

A mixture of 4 diatereo- and regioisomers in the crude NMR with a ratio of 16.6:5:5:1 were identified by $2 \mathrm{D}$ NMR.

$\mathbf{R}_{\mathbf{f}} 0.25$ (9:1, Hexane/Ethyl acetate)

${ }^{1} \mathbf{H}$ NMR $\left(400 \mathrm{MHz}, \mathrm{CDCl}_{3}\right) \delta$ 7.88-7.80 (m, $\left.3 \mathrm{H}\right), 7.76-7.69(\mathrm{~m}, 3 \mathrm{H}), 5.34(\mathrm{q}, 0.1 \mathrm{H}, J=9.8 \mathrm{~Hz})$, $5.12(\mathrm{~m}, 1 \mathrm{H}), 4.76(\mathrm{~m}, 0.25 \mathrm{H}), 4.46(\mathrm{~m}, 0.06 \mathrm{H}), 3.85-3.82(\mathrm{~m}, 3.5 \mathrm{H}), 3.80-3.74(\mathrm{~m}, 4.6 \mathrm{H}), 3.39$ (t, $0.05 \mathrm{H}, J=12.7 \mathrm{~Hz}), 3.38-3.26(\mathrm{~m}, 1.30 \mathrm{H}), 2.95(\mathrm{dd}, 1 \mathrm{H}, \mathrm{J}=12.7,11.3 \mathrm{~Hz}), 2.75(\mathrm{~m}, 0.06 \mathrm{H}), 2.64$ $(\mathrm{dd}, 1 \mathrm{H}, \mathrm{J}=14.5,7.0 \mathrm{~Hz}), 2.57-2.47(\mathrm{~m}, 0.9 \mathrm{H}), 2.47-2.40(\mathrm{dd}, 0.2 \mathrm{H}, J=14.6,5.7 \mathrm{~Hz}), 2.10(\mathrm{dd}, 1 \mathrm{H}$,

\footnotetext{
${ }^{16}$ not all the signals of the minor diastereoisomer were resolved by ${ }^{13} \mathrm{C}$.
} 
$\mathrm{J}=12.8,7.3 \mathrm{~Hz}), 1.91(\mathrm{~m}, 1 \mathrm{H}), 1.57(\mathrm{~m}, 0.3 \mathrm{H}), 1.53-1.26(\mathrm{~m}, 8 \mathrm{H}), 1.06-1.21(\mathrm{~m}, 30 \mathrm{H}), 0.90(\mathrm{t}, 4 \mathrm{H}$, $J=7.3 \mathrm{~Hz}), 0.75(\mathrm{t}, 0.9 \mathrm{H}, J=7.2 \mathrm{~Hz}), 0.67(\mathrm{t}, 0.3 \mathrm{H}, J=7.3 \mathrm{~Hz})$.

${ }^{13}$ C NMR $\left(101 \mathrm{MHz}, \mathrm{CDCl}_{3}\right) \delta 171.5,169.3,168.3,133.9,132.0,123.2,123.1,88.6,68.9,52.5,52.4$, 50.8, 47.4, 41.2, 36.6, 35.1, 34.6, 29.6, 28.1, 23.4, 22.3, 21.3, 18.6, 18.5, 18.4, 18.3, 14.4, 14.1, 13.9, $13.8^{15}$

IR 2952 (w), 2895 (w), 2868 (w), 1776 (w), 1741 (s), 1714 (s), 1377 (m), 1129 (m).

HRMS (ESI) calcd for $\mathrm{C}_{29} \mathrm{H}_{44} \mathrm{NO}_{7} \mathrm{Si}^{+}[\mathrm{M}+\mathrm{H}]^{+}$560.3038; found 560.3030

Dimethyl-4-(1,3-dioxoisoindolin-2-yl)-2-ethyl-3-methyl-2-((triisopropylsilyl)oxy)cyclopentane1,1-dicarboxylate (3fn):

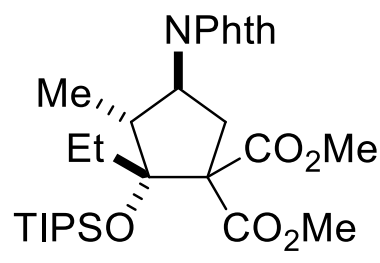

Using the method described above, $157 \mathrm{mg}(0.288 \mathrm{mmol}, 96 \%)$ of a colorless oil were obtained.

Starting from a 1:8 mixture of Z:E silyl enol ether, a mixture of 3 diastereomer with a ratio of 11:4:1 was identified by 2D NMR.

$\mathbf{R}_{\mathbf{f}} 0.7$ (6:4, Hexane/Ethyl acetate)

${ }^{1} \mathbf{H}$ NMR $\left(400 \mathrm{MHz}, \mathrm{CDCl}_{3}\right) \delta$ 7.88-7.81 (m, $\left.2 \mathrm{H}\right)$, 7.69-7.76 (m, $\left.3 \mathrm{H}\right), 4.86$-4.73 (m, $\left.1 \mathrm{H}\right), 4.55-4.43$ (m, $0 \mathrm{H}), 3.81-3.69(\mathrm{~m}, 8 \mathrm{H}), 3.54-3.40(\mathrm{~m}, 1 \mathrm{H}), 3.39-3.31(\mathrm{~m}, 1 \mathrm{H}), 3.08(\mathrm{dd}, 1 \mathrm{H}, \mathrm{J}=14.3,10.6 \mathrm{~Hz})$, 2.72-2.58 (m, 2 H), 2.36-2.20 (m, $1 \mathrm{H}), 1.99-1.88(\mathrm{~m}, 1 \mathrm{H}), 1.88-1.80(\mathrm{~m}, 0 \mathrm{H}), 1.23$ (t, $1 \mathrm{H}, \mathrm{J}=7.0$ $\mathrm{Hz}), 1.20-1.09(\mathrm{~m}, 29 \mathrm{H}), 1.09-0.96(\mathrm{~m}, 9 \mathrm{H})$

${ }^{13} \mathrm{C}$ NMR $\left(101 \mathrm{MHz}, \mathrm{CDCl}_{3}\right) \delta 171.6,171.3,169.6,169.5,168.6,168.3,133.9,132.0,131.8,123.2$, 123.1, 89.9, 87.8, 67.6, 67.2, 54.0, 52.6, 52.4, 52.4, 52.3, 48.5, 42.6, 35.1, 34.8, 30.5, 29.7, 29.4, 18.7, $18.6,18.5,18.4,18.0,14.6,14.1,13.4,13.3,11.3,9.9,9.7^{15}$

IR 2951 (w), 2869 (w), 1737 (m), 1715 (s), 1378 (m), $1266(\mathrm{w}), 1249$ (w), 1127 (m)

HRMS (ESI) calcd for $\mathrm{C}_{29} \mathrm{H}_{44} \mathrm{NO}_{7} \mathrm{Si}+[\mathrm{M}+\mathrm{H}]^{+} 546.2882$; found 546.2870

Dimethyl-3-(1,3-dioxoisoindolin-2-yl)-7a-((triisopropylsilyl)oxy)octahydro-1H-indene-1,1dicarboxylate (6fo):

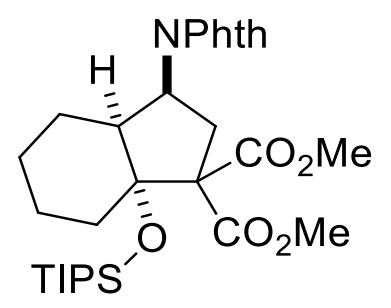

Using the method described above, $159 \mathrm{mg}(0.285 \mathrm{mmol}, 95 \%)$ of three diastereomers (14:3:1) as a colorless oil were obtained. The major diastereomer was separated from others by column chromatography.

The two enantiomers were separated by HPLC using Chiralpack IB column $(0.46 \times 25 \mathrm{~cm}), 98: 2$ Hexane/Isopropanol, $0.6 \mathrm{ml} / \mathrm{min} ; \operatorname{tr}_{1}=20.0 \mathrm{~min},[\alpha]_{\mathrm{D}}{ }^{25.0} 20.1\left(\mathrm{c}=0.5, \mathrm{CHCl}_{3}\right) ; \operatorname{tr}_{2}=24.4 \mathrm{~min}, \mathbf{R}_{\mathbf{f}} 0.70$ (6:4, Hexane/Ethyl acetate). 
${ }^{1} \mathbf{H}$ NMR $\left(400 \mathrm{MHz}, \mathrm{CDCl}_{3}\right) \delta 7.82(\mathrm{~m}, 2 \mathrm{H}, P h t h), 7.71$ (m, $2 \mathrm{H}$, Phth), 5.25 (ddd, $1 \mathrm{H}, J=10.1$, 10.1, $7.1 \mathrm{~Hz}, \mathrm{~N}-\mathrm{C}-\mathrm{H}$ ), 4.04 (dd, $1 \mathrm{H}, J=14.4,10.5 \mathrm{~Hz}, \mathrm{CH}_{2}$ cyclopentane), 3.78 (s, $3 \mathrm{H}, \mathrm{OMe}$ ), 3.74 (s, $3 \mathrm{H}, \mathrm{OMe}$ ), 3.03 (dd, $1 \mathrm{H}, J=14.4,9.8 \mathrm{~Hz}, \mathrm{CH}_{2}$ cyclopentane), $2.91\left(\mathrm{~m}, 1 \mathrm{H}, \mathrm{CH}_{2}\right), 2.38(\mathrm{~m}, 1 \mathrm{H}$, $\mathrm{CH}$-), 1.80-1.54 (m, $\left.4 \mathrm{H}, \mathrm{CH}_{2}\right), 1.54-1.41\left(\mathrm{~m}, 1 \mathrm{H}, \mathrm{CH}_{2}\right), 1.39-1.28\left(\mathrm{~m}, 1 \mathrm{H}, \mathrm{CH}_{2}\right), 1.23-1.09$ (m, $22 \mathrm{H}$, $\mathrm{TIPS}+\mathrm{CH}_{2}$ )

${ }^{13} \mathrm{C}$ NMR $\left(101 \mathrm{MHz}, \mathrm{CDCl}_{3}\right) \delta 170.2,169.3,169.2,133.9,131.9,123.1,87.1,68.0,52.8,52.8,52.6$, $52.5,34.4,29.9,23.9,23.3,23.2,18.4,18.6,13.7^{15}$

IR 2949 (w), 2867 (w), 1774 (w), 1739 (m), 1715 (s), 1373 (w), 1133 (w)

HRMS (ESI) calcd for $\mathrm{C}_{30} \mathrm{H}_{44} \mathrm{NO}_{7} \mathrm{Si}+[\mathrm{M}+\mathrm{H}]+558.2882$; found 558.2892.

Trans-Dimethyl-4-(1,3-dioxoisoindolin-2-yl)-2-((trimethylsilyl)oxy)cyclopentane-1,1dicarboxylate (6fp):<smiles>COCC1(C(OC)OC)C[C@H](c2ccccc2)C[C@H]1OC</smiles>

Using the method described above, $97.0 \mathrm{mg}(0.231 \mathrm{mmol}, 77 \%)$ of a single diastereomer as a colorless solid was obtained.

The two enantiomers were separated by HPLC using Chiralpack IA column $(0.46 \times 25 \mathrm{~cm}), 98: 2$ Hexane/Isopropanol, $1 \mathrm{ml} / \mathrm{min} ; \operatorname{tr}_{1}=19.8 \mathrm{~min} ; \operatorname{tr}_{2}=24.0 \mathrm{~min},[\alpha]_{\mathrm{D}}^{25.0}-51\left(\mathrm{c}=0.68, \mathrm{CHCl}_{3}\right)$

$\mathbf{R}_{\mathbf{f}} 0.40$ (6:4, Hexane/Ethyl acetate).

Mp $120.6{ }^{\circ} \mathrm{C}$.

${ }^{1} \mathbf{H}$ NMR $\left(400 \mathrm{MHz}, \mathrm{CDCl}_{3}\right) \delta 7.80(\mathrm{~m}, 2 \mathrm{H}$, Phth), 7.69 (m, $2 \mathrm{H}$, Phth), 5.11 (m, $1 \mathrm{H}, \mathrm{N}-\mathrm{C}-\mathrm{H}), 4.99$ (dd, $1 \mathrm{H}, J=4.1,1.8 \mathrm{~Hz}, O-C-H$ ), 3.82 (s, $3 \mathrm{H}, O M e$ ), 3.72 (s, $3 \mathrm{H}, O M e$ ), 3.14 (dd, $1 \mathrm{H}, J=14.4,10.7$ $\mathrm{Hz}, \mathrm{CH}_{2}$ ), 2.55 (m, $1 \mathrm{H}, \mathrm{CH}_{2}$ ), 2.46 (dd, $1 \mathrm{H}, J=14.5,6.6 \mathrm{~Hz}, \mathrm{CH}_{2}$ ), 1.98 (ddd, $1 \mathrm{H}, J=12.9,7.9,1.9$ $\left.\mathrm{Hz}, \mathrm{CH}_{2}\right), 0.11(\mathrm{~m}, 9 \mathrm{H}, \mathrm{TMS})$.

${ }^{13} \mathrm{C}$ NMR $\left(101 \mathrm{MHz}, \mathrm{CDCl}_{3}\right) \delta 171.3,169.0,168.0,134.0,131.9,123.2,76.4,66.0,52.8,52.5,47.6$, 38.7, 33.8, 0.0.

IR 2955 (w), 1775 (w), 1737 (s), 1710 (s), 1378 (m), 1251 (m), 1124 (m), 844 (s), 720 (s).

HRMS (ESI) calcd for $\mathrm{C}_{20} \mathrm{H}_{26} \mathrm{NO}_{7} \mathrm{Si}^{+}[\mathrm{M}+\mathrm{H}]^{+} 420.1473$; found 420.1483 .

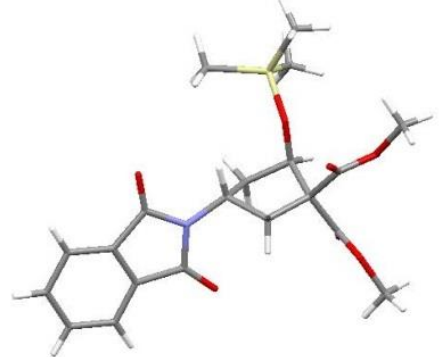

The crystal structure has been deposited at the Cambridge Crystallographic Data Centre and allocated the deposition number : CCDC 842234 


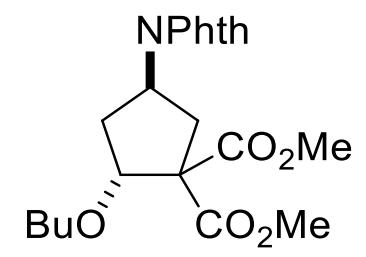

Using the method described above, $119 \mathrm{mg}(0.296 \mathrm{mmol}$, 99\%) of two unseparable diastereomers (20:1) as colorless oil were obtained.

Major diastereomer:

$\mathbf{R}_{\mathbf{f}} 0.5$ (6:4 Hexane/Ethyl acetate)

${ }^{1} \mathbf{H}$ NMR $\left(400 \mathrm{MHz}, \mathrm{CDCl}_{3}\right) \delta 7.84(\mathrm{~m}, 2 \mathrm{H}, \mathrm{Phth}), 7.72(\mathrm{~m}, 2 \mathrm{H}$, phth), 5.06 (m, $1 \mathrm{H}, \mathrm{N}-\mathrm{C}-\mathrm{H}), 4.64$ (dd, $1 \mathrm{H}, J=4.2,2.1 \mathrm{~Hz},-\mathrm{CH}-\mathrm{O}$ ), 3.87 (s, $3 \mathrm{H}, O M e$ ), 3.77 (s, $3 \mathrm{H}, O M e$ ), 3.57 (dt, $1 \mathrm{H}, J=9.2,6.1$ $\mathrm{Hz}, \mathrm{OCH}_{2} n$-butyl), 3.38 (dt, $1 \mathrm{H}, J=9.2,6.5 \mathrm{~Hz}, \mathrm{OCH}_{2} n$-butyl), 3.14 (dd, $1 \mathrm{H}, J=14.5,10.7 \mathrm{~Hz}$, $\left.\mathrm{CH}_{2}-\mathrm{C}\left(\mathrm{CO}_{2} \mathrm{Me}\right)_{2}\right), 2.54-2.42\left(\mathrm{~m}, 2 \mathrm{H}, \mathrm{CH}_{2}-\mathrm{CH}-\mathrm{O}-\right), 2.23$ (ddd, $1 \mathrm{H}, J=13.2,8.1,2.1 \mathrm{~Hz}, \mathrm{CH}_{2^{-}}$ $\left.\mathrm{C}\left(\mathrm{CO}_{2} \mathrm{Me}\right)_{2}\right), 1.59-1.45$ (m, $2 \mathrm{H}, \mathrm{CH}_{2} n$-butyl), 1.42-1.25 (m, $2 \mathrm{H}, \mathrm{CH}_{2} n$-butyl), 0.92 (t, $4 \mathrm{H}, J=7.4$ $\left.\mathrm{Hz}, \mathrm{CH}_{3}\right)$.

${ }^{13} \mathrm{C}$ NMR $\left(101 \mathrm{MHz}, \mathrm{CDCl}_{3}\right) \delta 171.3,169.0,168.0,134.0,131.9,123.2,83.1,69.6,64.9,52.9,52.6$, 47.4, 34.8, 34.1, 31.8, 19.3, 13.9

HRMS (ESI) calcd for $\mathrm{C}_{21} \mathrm{H}_{26} \mathrm{NO}_{7}^{+}[\mathrm{M}+\mathrm{H}]^{+}$404.1704; found 404.1720.

Dimethyl 5-(1,3-dioxoisoindolin-2-yl)hexahydrocyclopenta[b]pyran-7,7(7aH)dicarboxylate (6fr):<smiles>COC(=O)C1(C(C)=O)C[C@H](c2ccccc2)[C@H]2CCCO[C@H]21</smiles>

6fr-trans<smiles>COCC1(OC)CC([PH+]c2ccccc2)[C@H]2CCCO[C@H]21</smiles>

6fr-cis

Using the method described above, $115 \mathrm{mg}(0.297 \mathrm{mmol}, 99 \%)$ of two diastereomers (1.7:1) as a colorless solid was obtained. The two isomers were separated by column chromatography.

6fr-trans

Recrystallized in isopropanol

$\mathbf{R}_{\mathbf{f}} 0.13$ (6:4, Hexane/Ethyl acetate).

Mp $169.2^{\circ} \mathrm{C}$.

${ }^{1} \mathbf{H}$ NMR $\left(400 \mathrm{MHz}, \mathrm{CDCl}_{3}\right) \delta 7.82(\mathrm{~m}, 2 \mathrm{H}$, Phth), $7.72(\mathrm{~m}, 2 \mathrm{H}$, Phth), 4.87 (m, $1 \mathrm{H}, \mathrm{N}-\mathrm{C}-\mathrm{H}), 4.29$ $(\mathrm{d}, 1 \mathrm{H}, J=11.8 \mathrm{~Hz}, O-C-H), 4.05\left(\mathrm{dd}, 1 \mathrm{H}, J=11.5,4.5 \mathrm{~Hz}, C_{2}-O\right), 3.89$ (s, $3 \mathrm{H}, O M e$ ), 3.79 (s, 3 $\mathrm{H}, O M e), 3.51\left(\mathrm{dd}, 1 \mathrm{H}, J=12.1,12.1,3.0 \mathrm{~Hz}, \mathrm{CH}_{2}-\mathrm{O}\right), 3.04\left(\mathrm{dd}, 1 \mathrm{H}, J=15.2,9.6 \mathrm{~Hz}, \mathrm{CH}_{2}\right.$ cyclopentane), 2.70 (dd, $1 \mathrm{H}, J=15.2,2.7 \mathrm{~Hz}, \mathrm{CH}_{2}$ cyclopentane), 2.25 (m, $1 \mathrm{H}, \mathrm{CH}$ cyclopentane), 1.87 (m, $1 \mathrm{H}, \mathrm{CH}_{2}$ pyran), 1.68-1.49 (m, $2 \mathrm{H}, \mathrm{CH}_{2}$ pyran), 1.14 (qd, $1 \mathrm{H}, J=12.2,4.3 \mathrm{~Hz}, \mathrm{CH}_{2}$ pyran). ${ }^{13} \mathrm{C}$ NMR $\left(101 \mathrm{MHz}, \mathrm{CDCl}_{3}\right) \delta 171.8,171.0,168.8,134.2,131.5,123.4,84.2,69.2,61.8,53.1,53.0$, 49.2, 45.0, 35.0, 25.2, 25.0

IR 2951 (w), 2853 (w), 2255 (w), 1779 (w), 1749 (w), 1731 (m), 1708 (m), 1370 (m), 1276 (m), 723 (s), $648(\mathrm{~s}), 634(\mathrm{~s})$.

HRMS (ESI) calcd for $\mathrm{C}_{20} \mathrm{H}_{22} \mathrm{NO}_{7}^{+}[\mathrm{M}+\mathrm{H}]^{+} 388.1391$; found 388.1404 . 


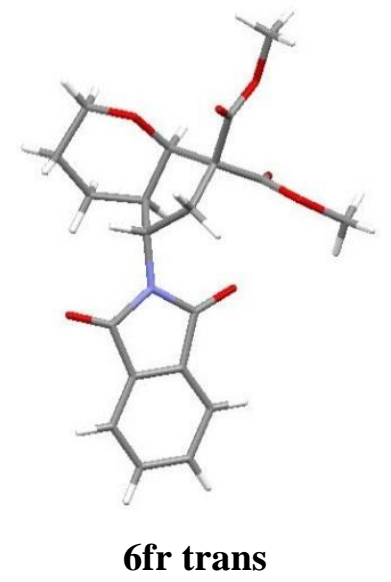

The crystal structure has been deposited at the Cambridge Crystallographic Data Centre and allocated the deposition number : CCDC 842235

\section{$\underline{\text { 6fr-cis }}$}

$\mathbf{R}_{\mathbf{f}} 0.31$ (6:4, Hexane/Ethyl acetate).

Mp $173.5^{\circ} \mathrm{C}$.

${ }^{1} \mathbf{H}$ NMR (400 MHz, $\left.\mathrm{CDCl}_{3}\right) \delta 7.83$ (m, $2 \mathrm{H}$, Phth), 7.72 (m, $2 \mathrm{H}$, Phth), 5.05 (ddd, $1 \mathrm{H}, J=10.9$, $10.9,6.6 \mathrm{~Hz}, \mathrm{~N}-\mathrm{C}-\mathrm{H}), 4.47$ (d, $1 \mathrm{H}, J=3.0 \mathrm{~Hz}, O-C-H), 4.00$ (dd, $1 \mathrm{H}, \mathrm{J}=11.4,4.4 \mathrm{~Hz}, \mathrm{CH}_{2}-\mathrm{O}$ ), 3.88 (s, $3 \mathrm{H}, O M e$ ), 3.77 (s, $3 \mathrm{H}, O M e$ ), 3.40 (dd, $1 \mathrm{H}, \mathrm{J}=12.2,2.1 \mathrm{~Hz}, \mathrm{CH}_{2}-\mathrm{O}$ ), 3.27 (dd, $1 \mathrm{H}, J=14.6$, $11.0 \mathrm{~Hz}, \mathrm{CH}_{2}$ cyclopentane), 2.87 (m, $1 \mathrm{H}, \mathrm{CH}$ cyclopentane), 2.64 (dd, $1 \mathrm{H}, J=14.6,6.5 \mathrm{~Hz}, \mathrm{CH}_{2}$ cyclopentane), 1.99 (m, $1 \mathrm{H}, \mathrm{CH}_{2}$ pyran), 1.74 (m, $1 \mathrm{H}, \mathrm{CH}_{2}$ pyran), 1.59 (m, $1 \mathrm{H}, \mathrm{CH}_{2}$ pyran), 1.40 (m, $1 \mathrm{H}, \mathrm{CH}_{2}$ pyran).

${ }^{13} \mathbf{C ~ N M R}\left(101 \mathrm{MHz}, \mathrm{CDCl}_{3}\right) \delta 171.0,168.8,168.2,134.0,131.9,123.2,82.1,67.9,63.9,52.9,52.9$, 49.0, 40.9, 33.0, 21.0, 20.0.

IR 2954 (w), 2858 (w), 1736 (s), 1710 (s), 1383 (m), 1108 (m), 912 (m), $719(\mathrm{~s})$

HRMS (ESI) calcd for $\mathrm{C}_{20} \mathrm{H}_{22} \mathrm{NO}_{7}^{+}[\mathrm{M}+\mathrm{H}]^{+} 388.1391$; found 388.1395 .

\section{Diethyl 2-(2-(1,3-dioxoisoindolin-2-yl)-4-0xo-4-phenylbutyl)malonate (7da):}<smiles></smiles>

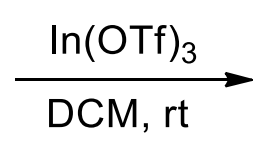<smiles>CCOC(=O)C(CC(CC(=O)c1ccccc1)Nc1ccccc1)C(=O)OCC</smiles>

Indium (III) trifluoromethanesulfonate $(17.0 \mathrm{mg}, 0.026 \mathrm{mmol}, 20 \mathrm{~mol} \%)$ is weighted in the glovebox. The flask is closed with a septum and put under $\mathrm{N}_{2}$ atmosphere. A solution of aminocyclopropane (50 $\mathrm{mg}, 0.15 \mathrm{mmol}, 1 \mathrm{eq})$ and silyl enol ether $(50 \mathrm{mg}, 0.18 \mathrm{mmol}, 1.2 \mathrm{eq})$ in $1 \mathrm{~mL}$ of dry dichloromethane is added. The reaction is stirred overnight at room temperature. The reaction is filtered through a plug of silica in order to remove the catalyst and concentrated under reduced pressure. Purification by column chromatograpy (6:4 Hexane/Ethyl Acetate) furnished $58 \mathrm{mg}(0.13 \mathrm{mmol}, 85 \%$ yield $)$ of the desired product as a colorless oil.

$\mathbf{R}_{\mathbf{f}} 0.32$ (6:4 Hexane/Ethyl Acetate). 
${ }^{1}$ H NMR $\left(400 \mathrm{MHz}, \mathrm{CDCl}_{3}\right) \delta 7.93(\mathrm{~m}, 2 \mathrm{H}, A r), 7.87-7.80$ (m, $2 \mathrm{H}$, Phth), 7.75-7.68 (m, $2 \mathrm{H}$, Phth), 7.59-7.53 (m, $1 \mathrm{H}, A r)$, 7.48-7.41 (m, $2 \mathrm{H}, A r), 5.02-4.94(\mathrm{~m}, 1 \mathrm{H}, N-C-H), 4.29-4.20(\mathrm{~m}, 2 \mathrm{H}$, $\left.\mathrm{CO}_{2} \mathrm{CH}_{2} \mathrm{CH}_{3}\right), 4.09-3.92\left(\mathrm{~m}, 3 \mathrm{H}, \mathrm{CO}_{2} \mathrm{CH}_{2} \mathrm{CH}_{3}+\mathrm{CH}\left(\mathrm{CO}_{2} \mathrm{Et}\right)_{2}\right), 3.51\left(\mathrm{dd}, 1 \mathrm{H}, J=17.7,5.3 \mathrm{~Hz}, \mathrm{CH}_{2^{-}}\right.$ $\mathrm{CO}-\mathrm{Ph}$ ), 3.40 (dd, $1 \mathrm{H}, J=8.3,6.2 \mathrm{~Hz}, \mathrm{CH}_{2}-\mathrm{CO}-\mathrm{Ph}$ ), 2.79 (ddd, $1 \mathrm{H}, J=14.3,11.0,6.2 \mathrm{~Hz}, \mathrm{CH}_{2}$ ), $2.47\left(\mathrm{ddd}, 1 \mathrm{H}, J=14.3,8.3,4.1 \mathrm{~Hz}, \mathrm{CH}_{2}\right), 1.29$ (t, $\left.3 \mathrm{H}, J=7.1 \mathrm{~Hz}, \mathrm{CO}_{2} \mathrm{CH}_{2} \mathrm{CH}_{3}\right), 1.17$ (t, $3 \mathrm{H}, J=7.1$ $\left.\mathrm{Hz}, \mathrm{CO}_{2} \mathrm{CH}_{2} \mathrm{CH}_{3}\right)$.

${ }^{13} \mathrm{C}$ NMR $\left(101 \mathrm{MHz}, \mathrm{CDCl}_{3}\right) \delta 196.7,168.7,168.4,168.2,136.5,134.0,133.4,131.8,128.7,128.1$, 123.3, 61.8, 61.6, 49.6, 45.6, 40.7, 31.6, 14.0, 13.9.

IR $2983(\mathrm{w}), 1776(\mathrm{w}), 1748(\mathrm{~m}), 1731(\mathrm{~s}), 1711(\mathrm{~s}), 1687(\mathrm{w}), 1393(\mathrm{w}), 1373(\mathrm{~m})$.

HRMS (ESI) calcd for $\mathrm{C}_{25} \mathrm{H}_{26} \mathrm{NO}_{7}^{+}[\mathrm{M}+\mathrm{H}]^{+} 452.1704$; found 452.1708

\section{dimethyl 2-(2-(1,3-dioxoisoindolin-2-yl)-3-methyl-4-oxo-4-phenylbutyl)malonate (7fji):}<smiles>COC(=O)C1(C(C)=O)CC1Nc1ccccc1</smiles>

PhthN<smiles>[O-]C(=C1C=CCC1)c1ccccc1</smiles><smiles>COC(=O)C(CC(NPc1ccccc1)[C@@H](C)C(=O)c1ccccc1)C(C)=O</smiles>

7fj

Following the same procedure described above, using $50 \mathrm{mg}(0.17 \mathrm{mmol}, 1 \mathrm{eq})$ of aminocyclopropane, $72 \mathrm{mg}(0.25 \mathrm{mmol}, 1.5 \mathrm{eq})$ of silyl enol ether and $18.5 \mathrm{mg}(0.033 \mathrm{mmol}, 20 \mathrm{~mol} \%)$ of Indium (III) trifluoromethanesulfonate, $49 \mathrm{mg}(0.11 \mathrm{mmol}, 67 \%$ yield $)$ of a colorless oil is obtained after column chromatography purification (9:1 Hexane/Ethyl Acetate to 8:2 Hexane/Ethyl Acetate).

4:1 Mixture of syn/anti diastereomers:

$\mathbf{R}_{\mathbf{f}} 0.29$ (6:4 Hexane/Ethyl Acetate).

${ }^{1} \mathbf{H}$ NMR $\left(400 \mathrm{MHz}, \mathrm{CDCl}_{3}\right) \delta$ 8.00-8.04 (m, $\left.2 \mathrm{H}, A r\right), 7.94-7.88(\mathrm{~m}, 2 \mathrm{H}$, Phth), 7.88-7.85 (m, 0.5 H, Phth), 7.81-7.76 (m, 2 H, Phth), 7.76-7.72 (m, 0.5 H, Phth), 7.68-7.60 (m, 1.5 H, Ar), 7.57-7.46 (m, $2.25 \mathrm{H}, A r), 7.42-7.35(\mathrm{~m}, 0.5 \mathrm{H}, A r), 4.83(\mathrm{dd}, 1 \mathrm{H}, J=10.7,3.2 \mathrm{~Hz}, N-C-H), 4.62(\mathrm{~m}, 0.5 \mathrm{H}, N-C-H$ + Me-CH-CO-Ph), 4.48 (qd, $1 \mathrm{H}, J=10.4,7.0 \mathrm{~Hz}, \mathrm{Me}-\mathrm{CH}-\mathrm{CO}-\mathrm{Ph}$ ), 3.79 (s, $0.75 \mathrm{H}, \mathrm{CO}_{2} \mathrm{CH}_{3}$ ), 3.76 $\left(\mathrm{s}, 3 \mathrm{H}, \mathrm{CO}_{2} \mathrm{CH}_{3}\right), 3.56\left(\mathrm{~s}, 0.75 \mathrm{H}, \mathrm{CO}_{2} \mathrm{CH}_{3}\right), 3.46\left(\mathrm{~s}, 3 \mathrm{H}, \mathrm{CO}_{2} \mathrm{CH}_{3}\right), 3.41-3.33(\mathrm{~m}, 1.25 \mathrm{H}$, $\left.\mathrm{CH}\left(\mathrm{CO}_{2} \mathrm{Et}\right)_{2}\right), 2.76$ (ddd, $\left.1 \mathrm{H}, J=14.4,11.4,6.7 \mathrm{~Hz}, \mathrm{CH}_{2}\right), 2.72-2.59\left(\mathrm{~m}, 0.5 \mathrm{H}, \mathrm{CH}_{2}\right), 2.23(\mathrm{ddd}, 1 \mathrm{H}$, $\left.J=14.4,7.9,3.2 \mathrm{~Hz}, \mathrm{CH}_{2}\right), 1.38\left(\mathrm{~d}, 0.75 \mathrm{H}, J=6.8 \mathrm{~Hz}, \mathrm{CH}_{3}\right), 1.09\left(\mathrm{~d}, 3 \mathrm{H}, J=7.1 \mathrm{~Hz}, \mathrm{CH}_{3}\right)$.

${ }^{13} \mathrm{C}$ NMR $\left(101 \mathrm{MHz}, \mathrm{CDCl}_{3}\right) \delta 201.8,201.2,169.2,169.1,168.8,168.6,168.5,136.1,135.9,134.3$, 134.0, 133.6, 133.2, 131.5, 131.4, 128.9, 128.7, 128.6, 128.4, 123.5, 123.3, 53.0, 52.9, 52.7, 52.5, $51.9,51.4,49.8,48.9,42.6,42.3,30.0,28.9,16.5,16.0$

IR 2955 (w), 1753 (m), 1753 (m), 1736 (s), 1710 (s), 1680 (m), 1389 (m), 1367 (m), 723 (s).

HRMS (ESI) calcd for $\mathrm{C} 24 \mathrm{H} 24 \mathrm{NO} 7+[\mathrm{M}+\mathrm{H}]+438.1547$; found 438.1556
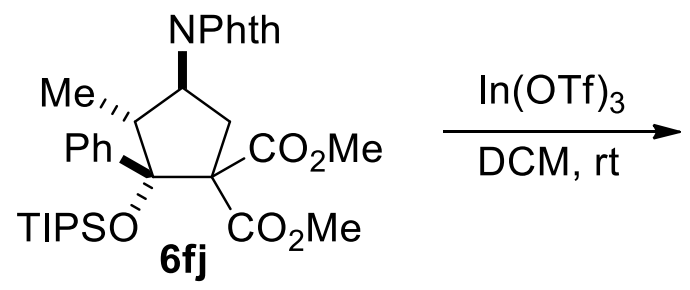<smiles>[CH][Pb](NC(CC(C)OC)[C@H](C)C(=O)c1ccccc1)c1ccccc1</smiles>

Indium (III) trifluoromethanesulfonate $(5 \mathrm{mg}, 0.009 \mathrm{mmol}, 20 \mathrm{~mol} \%$ ) is weighted in the glovebox. The flask is closed with a septum and put under $\mathrm{N}_{2}$ atmosphere. A solution of $\mathbf{6 f j} \mathbf{j}(26 \mathrm{mg}, 0.044 \mathrm{mmol}$, 
1 eq) in $0.5 \mathrm{~mL}$ of dry dichloromethane is added. The reaction is stirred overnight at room temperature. The reaction is filtered through a plug of silica in order to remove the catalyst and concentrated under reduced pressure. Purification by column chromatograpy (6:4 Hexane/Ethyl Acetate) furnished $15 \mathrm{mg}(0.043 \mathrm{mmol}, 78 \%$ yield $)$ of the desired product as a colorless oil.

dr $>20: 1$ syn/anti

$\mathbf{R}_{\mathbf{f}} 0.29$ (6:4 Hexane/Ethyl Acetate).

${ }^{1} \mathbf{H}$ NMR $\left(400 \mathrm{MHz}, \mathrm{CDCl}_{3}\right) \delta 8.07(\mathrm{~d}, 2 \mathrm{H}, J=7.4 \mathrm{~Hz}, A r), 7.91(\mathrm{~m}, 2 \mathrm{H}$, Phth), 7.79 (m, $2 \mathrm{H}$, Phth), $7.64(\mathrm{~m}, 1 \mathrm{H}, A r), 7.54$ (t, $2 \mathrm{H}, J=7.8 \mathrm{~Hz}, A r), 4.84$ (ddd, $1 \mathrm{H}, J=10.9,10.9,3.1 \mathrm{~Hz}, N-C-H), 4.48$ (m, $1 \mathrm{H}, \mathrm{Me}-\mathrm{CH}$-CO-Ph), 3.76 (s, $\left.3 \mathrm{H}, \mathrm{CO}_{2} \mathrm{CH}_{3}\right), 3.47$ (s, $\left.3 \mathrm{H}, \mathrm{CO}_{2} \mathrm{CH}_{3}\right), 3.39$ (dd, $1 \mathrm{H}, J=7.2,7.2$ $\left.\mathrm{Hz}, \mathrm{CH}\left(\mathrm{CO}_{2} \mathrm{Et}\right)_{2}\right), 2.77$ (ddd, $\left.1 \mathrm{H}, J=14.4,11.4,6.8 \mathrm{~Hz}, \mathrm{CH}_{2}\right), 2.24(\mathrm{ddd}, 1 \mathrm{H}, J=14.4,7.9,3.2 \mathrm{~Hz}$, $\left.\mathrm{CH}_{2}\right), 1.10\left(\mathrm{~d}, 3 \mathrm{H}, J=7.1 \mathrm{~Hz}, \mathrm{CH}_{3}\right)$

${ }^{13} \mathrm{C}$ NMR $\left(101 \mathrm{MHz}, \mathrm{CDCl}_{3}\right) \delta 201.2,169.1,168.6,168.4,136.1,134.3,133.6,131.5,128.9,128.6$, $123.5,52.8,52.5,51.9,49.8,42.3,30.0,16.0$

IR 2955 (w), 1754 (m), 1736 (s), 1712 (s), 1388 (m), 1368 (m), 723 (s)

HRMS (ESI) calcd for $\mathrm{C}_{24} \mathrm{H}_{24} \mathrm{NO}_{7}{ }^{+}[\mathrm{M}+\mathrm{H}]^{+} 438.1547$; found 438.1505 .

Dimethyl 2-(2-(1,3-dioxoisoindolin-2-yl)-4-0xo-4-phenylbutyl)malonate (7fa) :

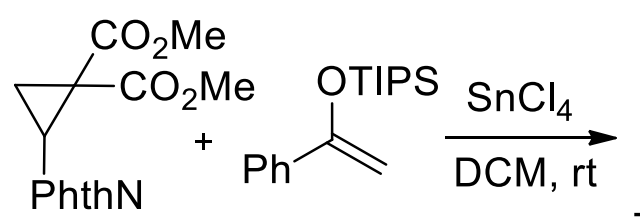<smiles>COC(=O)C(C)[C@H](C)O[SnH]</smiles>

3a: $72 \%$<smiles>COC(=O)C(CC(CC(=O)c1ccccc1)Nc1ccccc1)C(C)=O</smiles>

7fa: $24 \%$

To a solution of aminocyclopropane $(50 \mathrm{mg}, 0.17 \mathrm{mmol}, 1 \mathrm{eq})$ and silyl enol ether $(68 \mathrm{mg}, 0,25 \mathrm{mmol}$, $1.5 \mathrm{eq})$ in $1 \mathrm{~mL}$ of dry dichloromethane under nitrogen, is added $19.2 \mu \mathrm{l}(0.00826 \mathrm{mmol}, 5 \mathrm{~mol} \%)$ of a tin tetrachloride solution $(0.43 \mathrm{M})$ at room temperature. The reaction is stirred one hour at room temperature after what $0.1 \mathrm{~mL}$ of triethylamine is added. The solution is concentrated under reduced pressure and purified by column chromatography (9:1 to 8:2 Hexane/Ethyl Acetate). $69 \mathrm{mg}(0.12$ mmol, $72 \%)$ of $\mathbf{3 a}$ and $16.4(0.04 \mathrm{mmol}, 24 \%)$ of $\mathbf{7 f a}$ are isolated.

\section{$\underline{7 f a}$}

The two enantiomers were separated by HPLC using Chiralpack IA column $(0.46 \times 25 \mathrm{~cm}), 75: 25$ Hexane/Isopropanol, $1 \mathrm{ml} / \mathrm{min}$;

$-72.8 \%$ ee $\quad \operatorname{tr}_{1}=26.5 \mathrm{~min}[\alpha]_{\mathrm{D}}^{25.0}-27.3\left(\mathrm{c}=1, \mathrm{CHCl}_{3}\right) ; \operatorname{tr}_{2}=28.2 \mathrm{~min}$.

$+98 \%$ ee $\quad \operatorname{tr}_{1}=26.5 \mathrm{~min} ; \operatorname{tr}_{2}=28.2 \mathrm{~min} .[\alpha]_{\mathrm{D}}{ }^{25.0}+41\left(\mathrm{c}=0.6, \mathrm{CHCl}_{3}\right)$;

Sticky oil,

$\mathbf{R}_{\mathbf{f}} 0.45$ (6:4 Hexane/Ethyl Acetate).

${ }^{1} \mathbf{H}$ NMR $\left(400 \mathrm{MHz}, \mathrm{CDCl}_{3}\right) \delta$ 7.96-7.90 (m, $\left.2 \mathrm{H}, \mathrm{Ar}\right)$, 7.87-7.81 (m, $2 \mathrm{H}$, Phth), 7.76-7.70 (m, $2 \mathrm{H}$, Phth), 7.59-7.54 (m, $1 \mathrm{H}, A r), 7.49-7.43$ (m, $2 \mathrm{H}, A r)$, 5.02-4.92 (m, $1 \mathrm{H}, N-C-H), 4.02$ (dd, $1 \mathrm{H}, J=$ $\left.17.9,8.7 \mathrm{~Hz}, \mathrm{CH}\left(\mathrm{CO}_{2} \mathrm{Et}\right)_{2}\right), 3.80$ (s, $\left.3 \mathrm{H}, \mathrm{CO}_{2} \mathrm{CH}_{3}\right), 3.57$ (s, $\left.3 \mathrm{H}, \mathrm{CO}_{2} \mathrm{CH}_{3}\right), 3.51$ (dd, $1 \mathrm{H}, J=17.8,5.3$ $\mathrm{Hz}, \mathrm{CH}_{2}-\mathrm{CO}-\mathrm{Ph}$ ), 3.45 (dd, $1 \mathrm{H}, J=8.5,6.1 \mathrm{~Hz}, \mathrm{CH}_{2}-\mathrm{CO}-\mathrm{Ph}$ ), $2.80(\mathrm{ddd}, 1 \mathrm{H}, J=14.3,11.2,6.1 \mathrm{~Hz}$, $\left.\mathrm{CH}_{2}\right), 2.48\left(\mathrm{ddd}, 1 \mathrm{H}, J=14.3,8.6,3.9 \mathrm{~Hz}, \mathrm{CH}_{2}\right.$ ). 
${ }^{13} \mathrm{C}$ NMR $\left(101 \mathrm{MHz}, \mathrm{CDCl}_{3}\right) \delta 196.7,169.1,168.8,168.2,136.4,134.1,133.5,131.7,128.7,128.1$, $123.4,52.9,52.7,49.2,45.5,40.7,31.7$.

IR 2956 (w), 1774 (w), 1753 (m), 1734 (s), 1709 (s), 1687 (w), 1373 (m), 1372 (m), 724 (m).

HRMS (ESI) calcd for $\mathrm{C} 23 \mathrm{H} 22 \mathrm{NO} 7+[\mathrm{M}+\mathrm{H}]+424.1391$; found 424.1386

$\underline{\text { Trans-dimethyl 4-amino-2-phenyl-2-((triisopropylsilyl)oxy)cyclopentane-1,1-dicarboxylate }(8) \text { : }}$

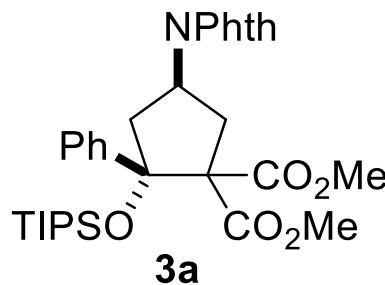

$3 a$

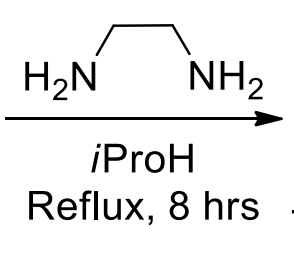

Reflux, $8 \mathrm{hrs}$<smiles>COC(OC)[C@]1(OC(C)=O)C[C@@H](N)C[C@]1(O)c1ccccc1</smiles>

8

Following a modified procedure ${ }^{17}, 100 \mathrm{mg}$ of $\mathbf{3 a}(0.172 \mathrm{mmol}, 1 \mathrm{eq})$ is added in an oven dried flask with $52 \mathrm{mg}(0.86 \mathrm{mmol}, 5 \mathrm{eq})$ of ethylenediamine. Isopropanol is added and the white suspension is refluxed. After a few minutes, the starting material is completely solubilized and the reaction is heated during $8 \mathrm{~h}$. The solvent is then removed under reduced pressure and the crude oil is purified by column chromatography (9:1 Ethyl Acetate/Hexane + 1\% NEt $) .55 \mathrm{mg}(0.12 \mathrm{mmol}, 72 \%)$ of a colorless oil corresponding to the free cyclopentylamine is isolated.

$\mathbf{R}_{\mathbf{f}} 0.2(100 \%$ Ethyl Acetate $)$

Mp $200^{\circ} \mathrm{C}$ decomposition

${ }^{1} \mathbf{H}$ NMR $\left(400 \mathrm{MHz}, \mathrm{CDCl}_{3}\right) \delta$ 7.78-7.74 (m, $\left.2 \mathrm{H}, A r\right), 7.32-7.24(\mathrm{~m}, 3 \mathrm{H}, \mathrm{Ar}), 3.82-3.82(\mathrm{~m}, 1 \mathrm{H}, \mathrm{N}$ $\mathrm{C}-\mathrm{H}$ ), 3.77 (s, $3 \mathrm{H}, \mathrm{CO}_{2} \mathrm{CH}_{3}$ ), 3.31 (s, $3 \mathrm{H}, \mathrm{CO}_{2} \mathrm{CH}_{3}$ ), 3.17 (dd, $1 \mathrm{H}, \mathrm{J}=13.9,8.7 \mathrm{H}, \mathrm{CH}_{2}$ ), 2.73 (dd, 1 $\left.\mathrm{H}, J=13.5,9.3 \mathrm{~Hz}, \mathrm{CH}_{2}\right), 2.61\left(\mathrm{dd}, 1 \mathrm{H}, J=13.4,6.9 \mathrm{~Hz}, \mathrm{CH}_{2}\right), 1.99\left(\mathrm{dd}, 1 \mathrm{H}, \mathrm{J}=14.0,6.2 \mathrm{~Hz}, \mathrm{CH}_{2}\right)$, 1.76 (br s, $2 \mathrm{H}, \mathrm{NH}_{2}$ ), 0.98-0.91 (m, $21 \mathrm{H}$, TIPS).

${ }^{13} \mathrm{C}$ NMR $\left(101 \mathrm{MHz}, \mathrm{CDCl}_{3}\right) \delta 172.1,169.2,142.0,128.0,127.8,127.2,89.0,71.3,52.1,49.4,49.0$, 44.6, 29.7, 18.2, 13.8

IR 2949 (w), 2949 (w), 2949 (w), 2949 (w), 2868 (w), 1752 (s), 1736 (s), 1717 (s), 1448 (w), 1263 (m), 1106 (s), 1077 (s)

HRMS (ESI) calcd for $\mathrm{C}_{24} \mathrm{H}_{40} \mathrm{NO}_{5} \mathrm{Si}^{+}[\mathrm{M}+\mathrm{H}]^{+} 450.2670$; found 450.2660

\section{Dimethyl 4-(1,3-dioxoisoindolin-2-yl)-2-phenylcyclopent-2-ene-1,1-dicarboxylate (9) :}

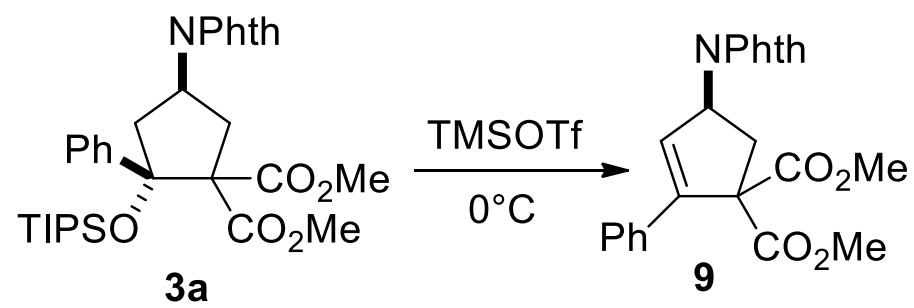

3a (80 mg, $0.14 \mathrm{mmol}, 1 \mathrm{eq})$ is added in an oven dried flask, under nitrogen. $1 \mathrm{~mL}$ of dry dichloromethane is added and the solution is cooled to $0{ }^{\circ} \mathrm{C}$ with an ice/water bath. TMSOTf $(28 \mu \mathrm{l}$, $0.15 \mathrm{mmol}, 1.1 \mathrm{eq}$ ) is added and the reaction is stirred for 10 minutes at $0{ }^{\circ} \mathrm{C}$. The solvent is evaporated under reduced pressure and the crude is purified by column chromatography $(8: 2$ Hexane/Ethyl Acetate). $30 \mathrm{mg}$ of a colorless solid (0.074 mmol, $53 \%$ yield) is isolated.

${ }^{17}$ O. Kanie, S. C. Crawley, M. M. Palcic, O. Hindsgaul, Carbohydrate Research 1993, 243, 139 
$\mathbf{R}_{\mathbf{f}} 0.43$ (6:4 Hexane/Ethyl Acetate).

Mp $132{ }^{\circ} \mathrm{C}$.

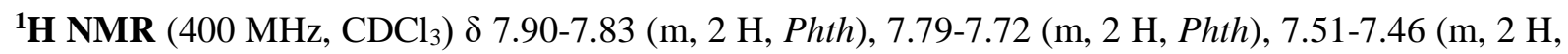
$A r)$, 7.37-7.29 (m, $3 \mathrm{H}, A r), 6.25(\mathrm{~d}, 1 \mathrm{H}, J=2.3 \mathrm{~Hz}, \mathrm{C}=\mathrm{CH}), 5.65-5.58(\mathrm{~m}, 1 \mathrm{H}, N-C-H), 3.82(\mathrm{~s}, 3 \mathrm{H}$, $\left.\mathrm{CO}_{2} \mathrm{CH}_{3}\right), 3.72\left(\mathrm{~s}, 3 \mathrm{H}, \mathrm{CO}_{2} \mathrm{CH}_{3}\right), 3.15\left(\mathrm{~m}, 2 \mathrm{H}, \mathrm{CH}_{2}\right)$.

${ }^{13} \mathrm{C}$ NMR $\left(101 \mathrm{MHz}, \mathrm{CDCl}_{3}\right) \delta 171.6,170.5,167.9,143.9,134.6,134.1,131.9,131.3,128.0,128.0$, $127.7,123.4,53.5,53.1,52.8,40.3,29.7$.

IR 1773 (w), 1732 (m), 1715 (s), 1391 (w), 1368 (w), 1273 (w), 1124 (w), 720 (m).

HRMS (ESI) calcd for $\mathrm{C}_{23} \mathrm{H}_{20} \mathrm{NO}_{6}{ }^{+}[\mathrm{M}+\mathrm{H}]^{+} 406.1285$; found 406.1295

Methyl 4-(1,3-dioxoisoindolin-2-yl)-2-phenylcyclopent-1-enecarboxylate (10):

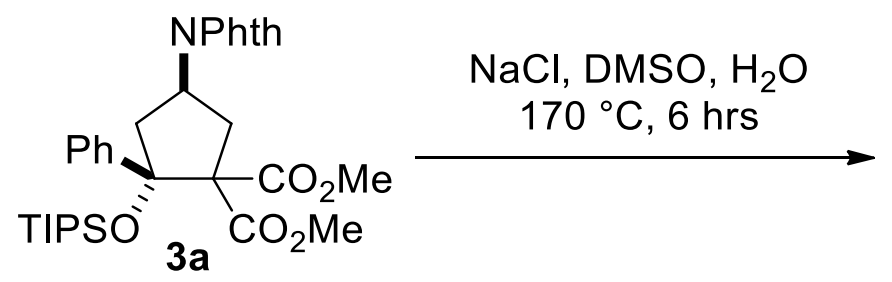<smiles>COC(OC)C1=C(c2ccccc2)C[C@H](Pc2ccccc2)C1</smiles>

Following a modified procedure ${ }^{18}, 3 \mathbf{a}(90 \mathrm{mg}, 0.15 \mathrm{mmol}, 1 \mathrm{eq})$ is dissolved is $2 \mathrm{~mL}$ dimethylsulfoxide and $5 \mu \mathrm{l}(0.3 \mathrm{mmol}, 2 \mathrm{eq})$ of water. The solution is stirred for $6 \mathrm{~h}$ at $170{ }^{\circ} \mathrm{C}$. After cooling to room temperature, the reaction mixture is extracted three times with $5 \mathrm{~mL}$ of diethyl ether, washed three times with $5 \mathrm{~mL}$ of water, three times with $5 \mathrm{~mL}$ brine dried over magnesium sulfate and filtered through a cotton plug. The solvents are evaporated under reduced pressure and the crude is purified by column chromatography ( $8: 2 \mathrm{Hexane} /$ Ethyl Acetate). $47 \mathrm{mg}(0.14 \mathrm{mmol}, 88 \%$ yield) of a white solid is isolated

$\mathbf{R}_{\mathbf{f}} 0.51$ (6:4 Hexane/Ethyl Acetate).

Mp $165^{\circ} \mathrm{C}$.

${ }^{1} \mathbf{H ~ N M R}^{19}\left(400 \mathrm{MHz}, \mathrm{CDCl}_{3}\right) \delta 7.88(\mathrm{~m}, 2 \mathrm{H}$, Phth), 7.75 (m, $2 \mathrm{H}$, Phth), 7.31-7.44 (m, $5 \mathrm{H}$, Ar), 5.14 (m, $1 \mathrm{H}, \mathrm{N}-\mathrm{C}-\mathrm{H}), 3.66\left(\mathrm{~s}, 3 \mathrm{H}, \mathrm{CO}_{2} \mathrm{CH}_{3}\right), 3.50\left(\mathrm{~m}, 1 \mathrm{H}, \mathrm{CH}_{2}\right), 3.35\left(\mathrm{~m}, 1 \mathrm{H}, \mathrm{CH}_{2}\right), 3.19\left(\mathrm{~m}, 2 \mathrm{H}, \mathrm{CH}_{2}\right)$ ${ }^{13} \mathbf{C ~ N M R}^{19}\left(101 \mathrm{MHz}, \mathrm{CDCl}_{3}\right) \delta 171.5,168.6,154.4,138.0,135.9,133.7,129.8,129.4,129.3,128.1$, $124.6,49.3,44.5,41.3,36.5$

IR 2949 (w), 1773 (w), 1710 (s), 1393 (m), 1378 (m), 1236 (m), 722 (m).

HRMS (ESI) calcd for $\mathrm{C}_{21} \mathrm{H}_{17} \mathrm{NNaO}_{4}{ }^{+}[\mathrm{M}+\mathrm{Na}]^{+} 370.1050$; found 370.1063

\footnotetext{
${ }^{18}$ A. P. Krapcho, J. F. Weimaster, J. M. Eldridge, E. G. E. Jahngen Jr., A. J. Lovey, W. P. Stephens, J. Org. Chem., 1978, 43, 138

${ }^{19}$ Peaks are splitting due to conformers of methyl ester.
} 


\section{$\underline{\text { Enantiospecific Reactions }}$}

\section{General procedure for enantiospecific cycloaddition :}

In an oven-dried flask sealed with a septum and under $\mathrm{N}_{2}$ atmosphere is added the chiral $\mathrm{N}$ phthalimide aminocyclopropane (16 mg $-26 \mathrm{mg}, 0.053-0.062 \mathrm{mmol}, 1 \mathrm{eq})$ and the silyl-enol ether (1.5 eq) in dry dichloromethane $(0.15 \mathrm{M})$. The solution is cooled down to $-78{ }^{\circ} \mathrm{C}$ and a $0.43 \mathrm{M}$ solution of tin tetrachloride $(10 \mathrm{~mol} \%)$ in dry dichloromethane is added. The reaction is stirred for $1 \mathrm{~h}$ at -78 ${ }^{\circ} \mathrm{C}$. Triethylamine $(0.1 \mathrm{~mL})$ is then added in one portion at $-78{ }^{\circ} \mathrm{C}$. The reaction is warmed at room temperature and stirred for $15 \mathrm{~min}$. Dichloromethane is removed under reduced pressure and the crude is directly purified by column chromatography (8:2 Hexane/Ethyl Acetate).<smiles>COC(=O)C1(C(=O)OC)CC1[PH2+]</smiles>

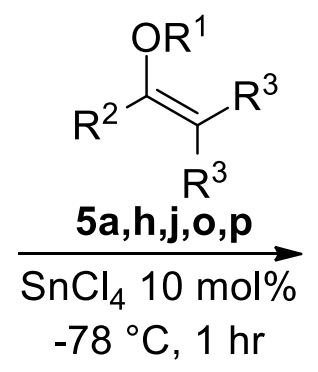

\begin{tabular}{ccccccc}
\hline Entry & \%ee $\mathbf{4 f}$ & $\mathbf{5}$ & $\mathbf{6}$ & Yield $^{\text {a }}$ & $d r^{\text {a }}$ & $\%$ ee $\mathbf{6}$ \\
\hline 5 & +97.4 & $\mathbf{5 a}$ & $\mathbf{6 f a}$ & 82 & $>20: 1$ & 98 \\
\hline 1 & +98.6 & $\mathbf{5 h}$ & $\mathbf{6 f h}$ & 73 & $>20: 1$ & 81 \\
\hline 2 & -94.8 & $\mathbf{5 j}$ & $\mathbf{6 f j}$ & 76 & $3: 1$ & 94 \\
\hline 3 & +98.9 & $\mathbf{5 0}$ & $\mathbf{6 f o}$ & 77.5 & $3: 1: 1$ & $99.2: 99.1: 98.9$ \\
\hline 4 & -97.6 & $\mathbf{5 p}$ & $\mathbf{6 f p}$ & 74 & $>20: 1$ & 95.7 \\
\hline
\end{tabular}

Indium catalysed synthesis of enantioenriched Dimethyl 2-(2-(1,3-dioxoisoindolin-2-yl)-4-oxo-4phenylbutyl)malonate (7fa):

Indium (III) trifluoromethanesulfonate $(3.3 \mathrm{mg}, 0.018 \mathrm{mmol}, 20 \mathrm{~mol} \%)$ is weighted in the glovebox. The flask is closed with a septum and put under $\mathrm{N}_{2}$ atmosphere. A solution of aminocyclopropane (18 $\mathrm{mg}, 0.059 \mathrm{mmol}, 1 \mathrm{eq})$ and silyl enol ether $(20 \mathrm{mg}, 0.071 \mathrm{mmol}, 1.2 \mathrm{eq})$ in dry dichloromethane (0.15 $\mathrm{M})$ is added. The reaction is stirred overnight at room temperature. The reaction is filtered through a plug of silica in order to remove the catalyst and concentrated under reduced pressure. Purification by column chromatograpy (6:4 Hexane/Ethyl Acetate) furnished $24 \mathrm{mg}(0.058 \mathrm{mmol}, 94 \%$ yield) of the desired product as a colorless oil.<smiles>[C+]C1(c2ccccc2)CC1(OC(C)=O)C(C)=O</smiles>

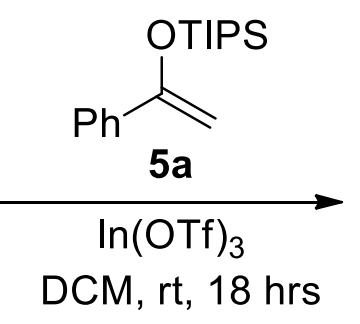<smiles>COCC(CC(CC(=O)c1ccccc1)NPc1ccccc1)C(=O)OC</smiles>

\begin{tabular}{ccc}
\hline \%ee 4f & Yield \% $^{\mathrm{a}}$ & \%ee 7fa \\
\hline-94 & 94 & -72.8 \\
\hline
\end{tabular}




\section{Opening of enantioenriched cyclopentylamines:}

Indium (III) trifluoromethanesulfonate $(3.5 \mathrm{mg}, 0.0062 \mathrm{mmol}, 20 \mathrm{~mol} \%)$ is weighted in the glovebox. The flask is closed with a septum and put under $\mathrm{N}_{2}$ atmosphere. A solution of $\mathbf{6 f a}(18.1 \mathrm{mg}, 0.031$ mmol, 1 eq) in $0.3 \mathrm{~mL}$ of dry dichloromethane $(0.15 \mathrm{M})$ is added. The reaction is stirred overnight at room temperature. The reaction is filtered through a plug of silica in order to remove the catalyst and concentrated under reduced pressure. Purification by column chromatography (6:4 Hexane/Ethyl Acetate) furnished $7.2 \mathrm{mg}(0.017 \mathrm{mmol}, 55 \%$ yield $)$ of the desired product as a colorless oil.

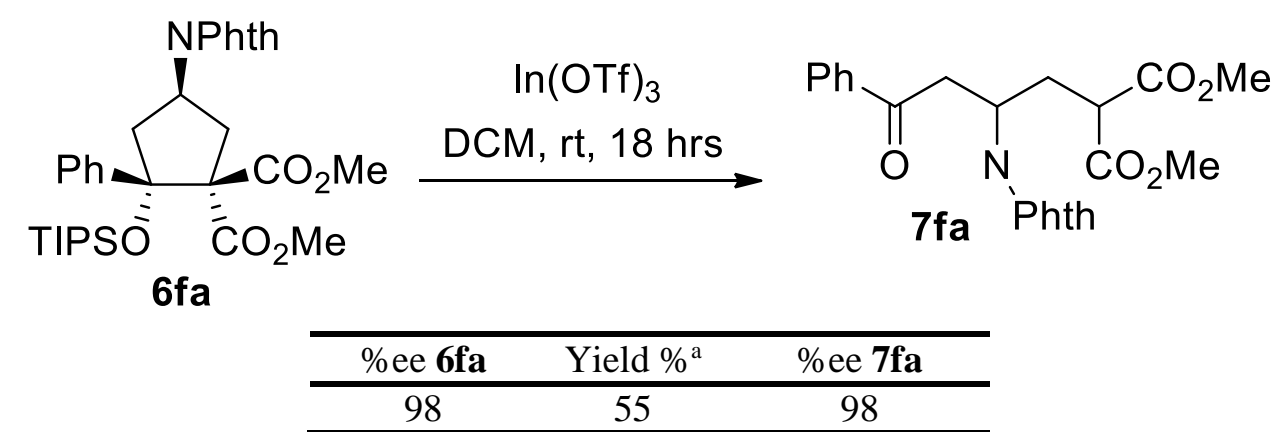




\section{Control Experiments}

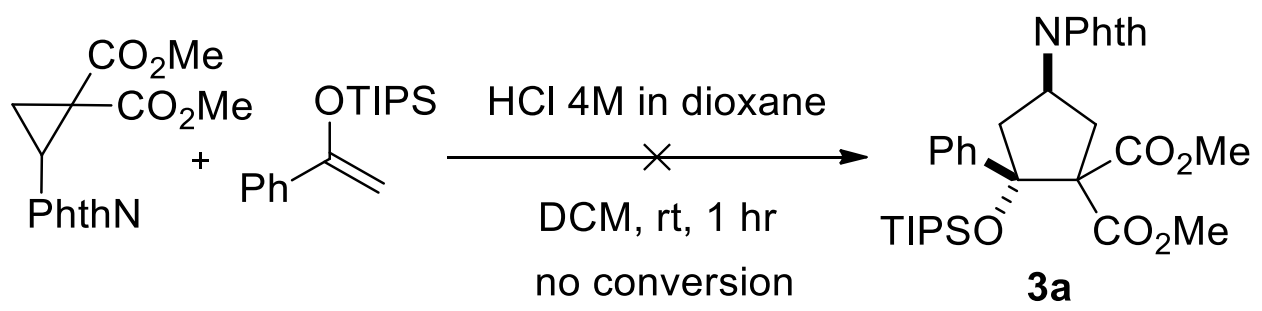

To a solution of aminocyclopropane (30 mg, $0.098 \mathrm{mmol}, 1 \mathrm{eq}$ ) and silyl enol ether (41 $\mathrm{mg}, 0.15$ $\mathrm{mmol}, 1.5 \mathrm{eq})$ in $0.3 \mathrm{~mL}$ of dry dichloromethane under nitrogen, is added $5.0 \mu \mathrm{l}(0.019 \mathrm{mmol}, 20 \mathrm{~mol}$ $\%$ ) of a $4 \mathrm{M} \mathrm{HCl}$ in dioxane solution at room temperature. The reaction is stirred at room temperature for 12 hours and no evolution of the starting material could be observed.<smiles>COC(=O)C1(C(C)OC)CC1[NH2+]c1ccccc1</smiles><smiles>C=C([OH+])[PH2+]S(=O)([O-])=S</smiles>

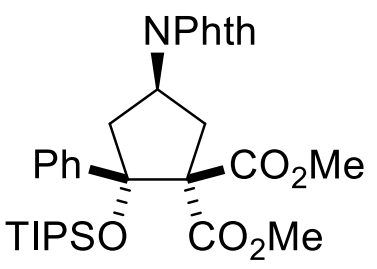

In an oven-dried flask sealed with a septum and under $\mathrm{N}_{2}$ atmosphere is added $50 \mathrm{mg}$ of $\mathrm{N}$ phthalimide aminocyclopropane $(0.16 \mathrm{mmol}, 1 \mathrm{eq}), 68 \mathrm{mg}$ of silyl-enol ether $(0.25 \mathrm{mmol}, 1.5 \mathrm{eq})$ and $3.1 \mathrm{mg}$ of 2,6-Di-tert-butylpyridine $(0.016 \mathrm{mmol}, 10 \mathrm{~mol} \%)$ in $0.5 \mathrm{~mL}$ of dry dichloromethane. The solution is cooled down to $-78{ }^{\circ} \mathrm{C}$ and $19.2 \mu \mathrm{L}$ of a $0.43 \mathrm{M}$ solution of tin tetrachloride $(5 \mathrm{~mol} \%)$ in dry dichloromethane is added. The reaction is stirred for $1 \mathrm{~h}$ at $-78{ }^{\circ} \mathrm{C}$. Triethylamine $(0.1 \mathrm{~mL})$ is then added in one portion at $-78{ }^{\circ} \mathrm{C}$. The reaction is warmed at room temperature and stirred for $15 \mathrm{~min}$. Dichloromethane is removed under reduced pressure and the crude is analyzed by ${ }^{1} \mathrm{H}$ NMR.

Ratio of starting material/product $=2.5: 1$

\section{Spectra of New Compounds}


solvent: $<C D C 13>$
Frequency $400.13 \mathrm{MH}$
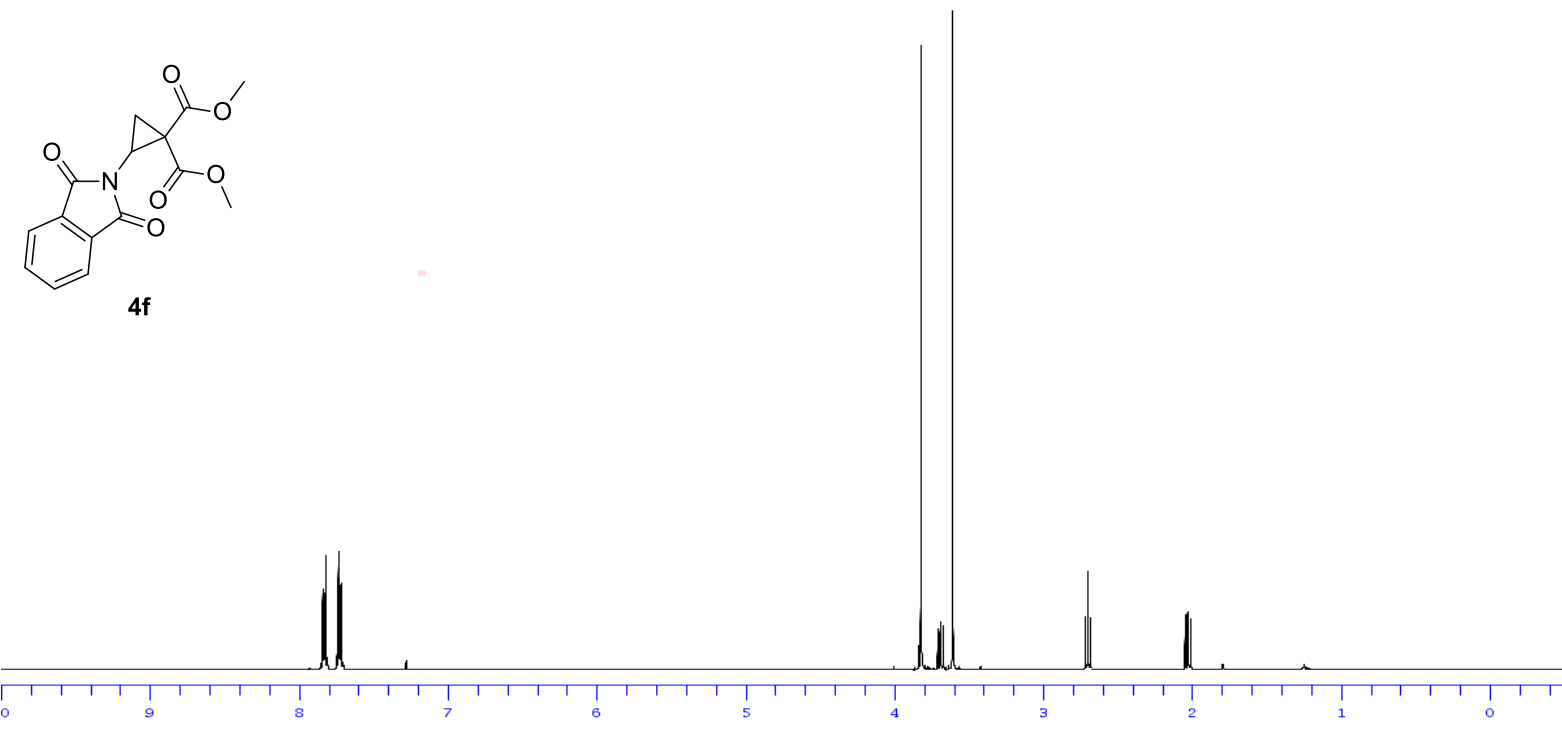

Solvent: $<C D C l 3>$
Frequency: $100.612769 \mathrm{MHz}$
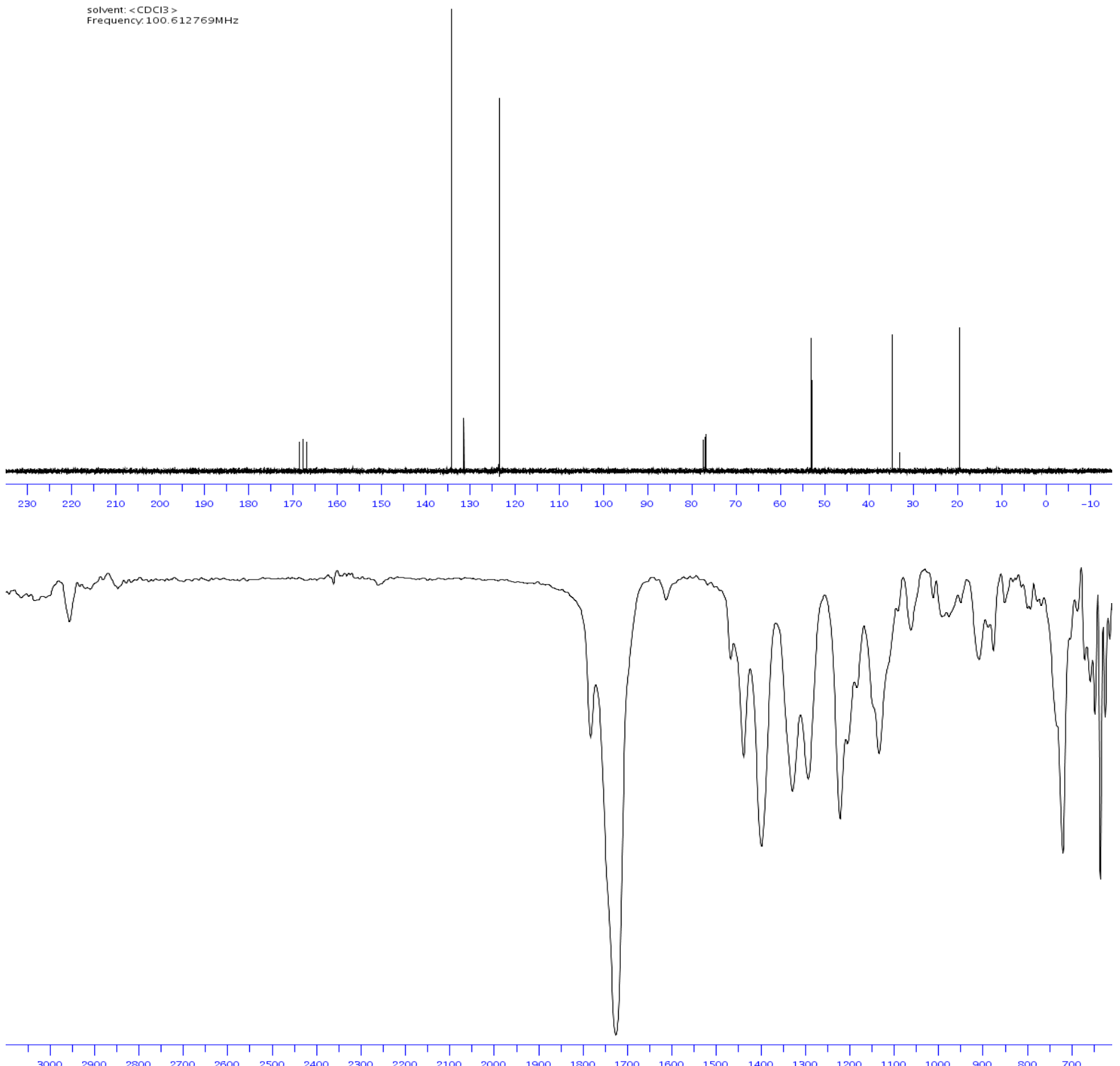
Solvent: $\langle C D C I 3\rangle$
Frequency: $400.13 \mathrm{MH}$

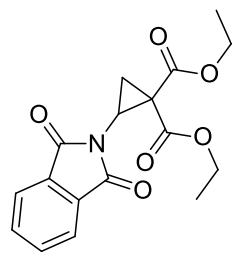

4d
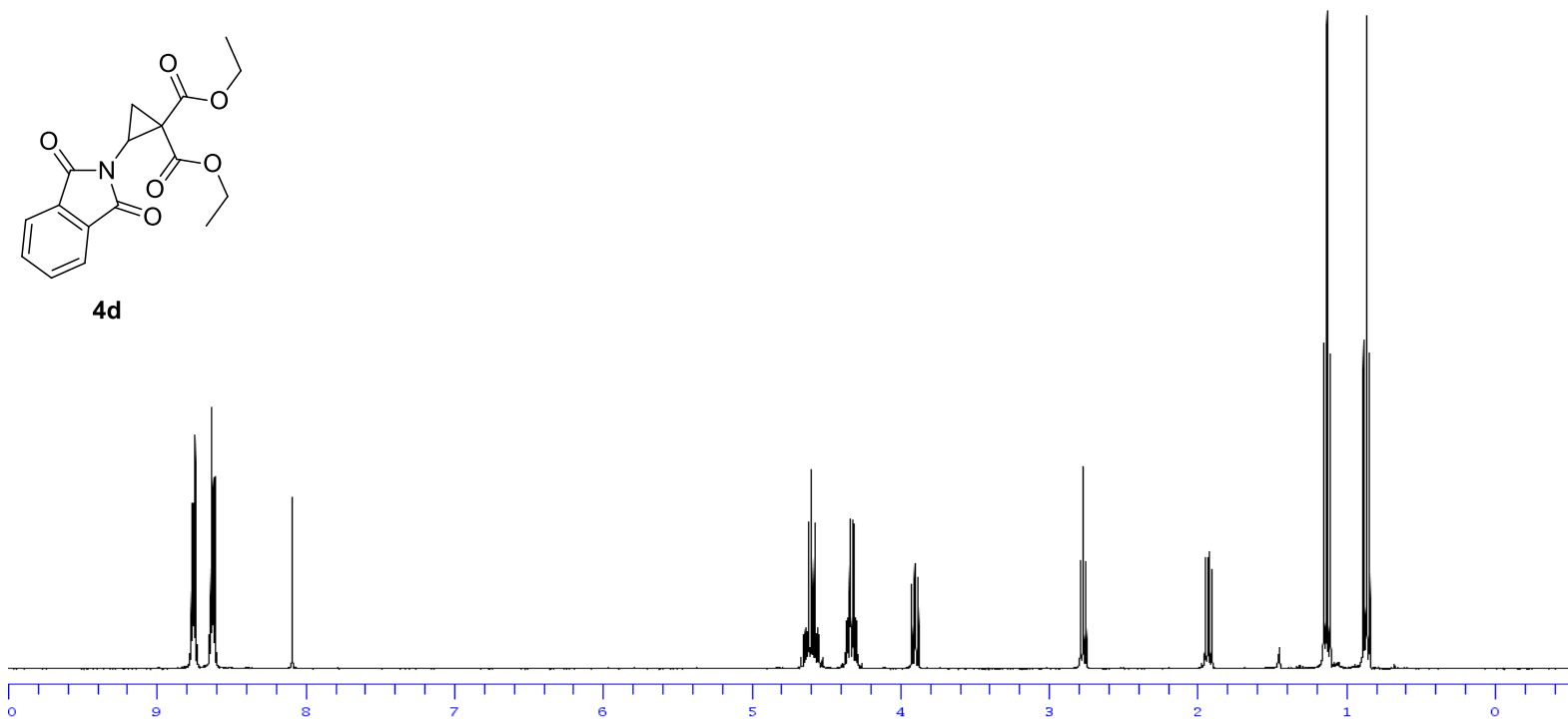

Solvent: $<C D C 13>$
Frequency: $100.612769 \mathrm{MH}$
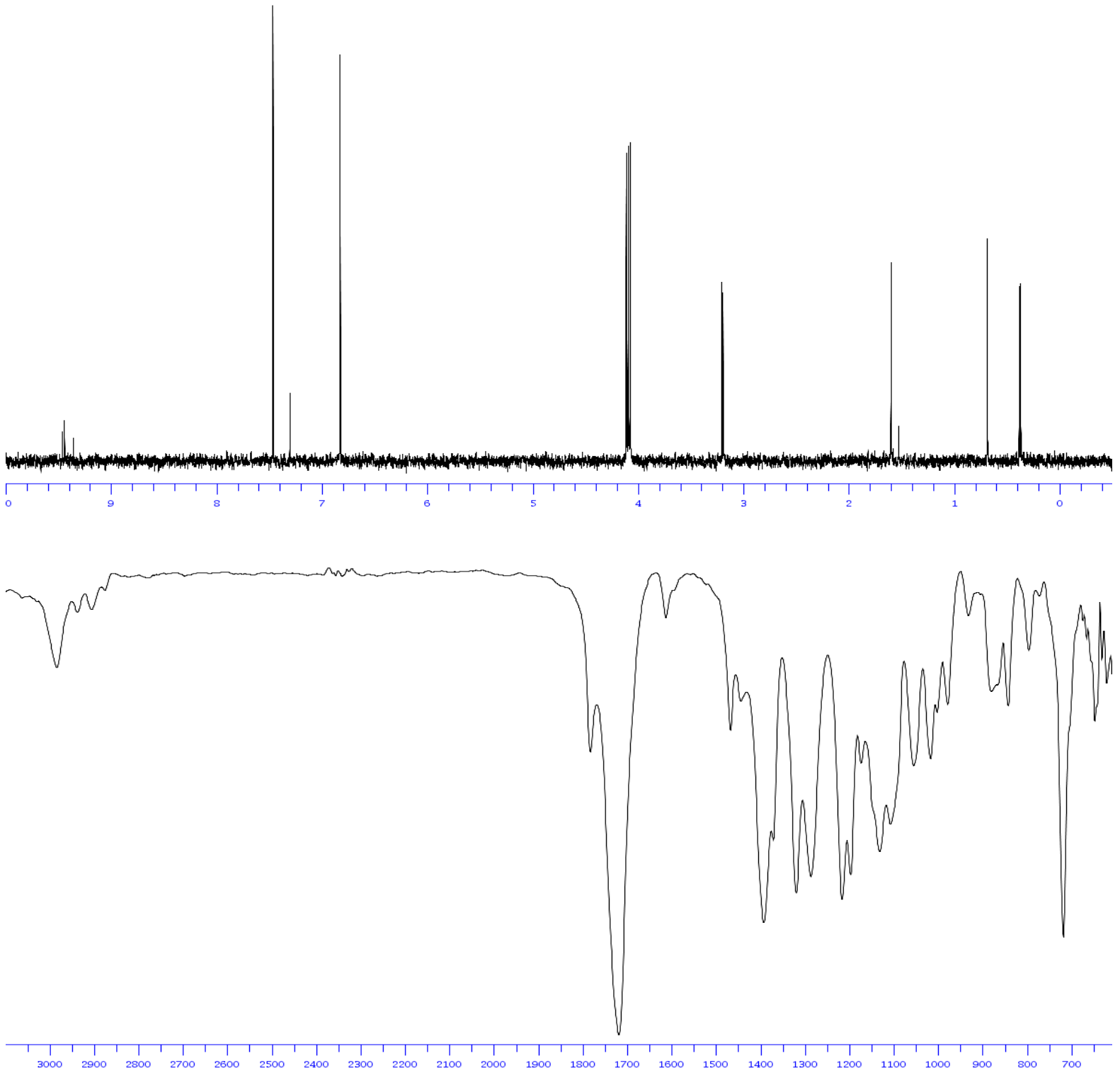
Solvent: $\langle\mathrm{CDCl} 3\rangle$
Frequency: $400.13 \mathrm{MH}$<smiles>CCOC(=O)C1CC1N1CCOC1=O</smiles>

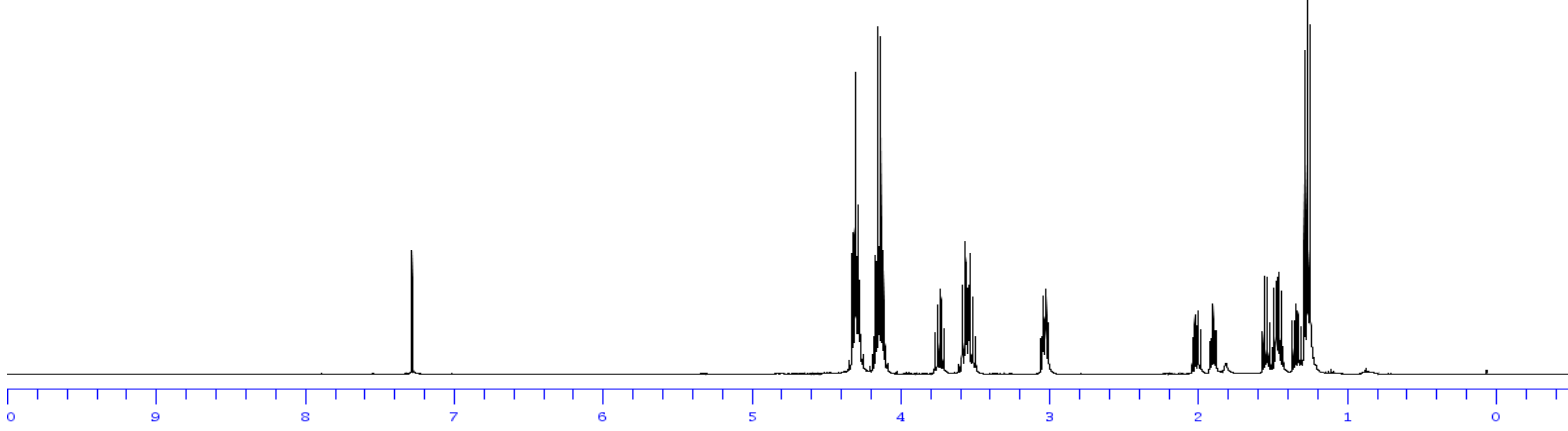

Solvent: $\angle C D C 13>$
Frequency: $100.612769 \mathrm{MHz}$
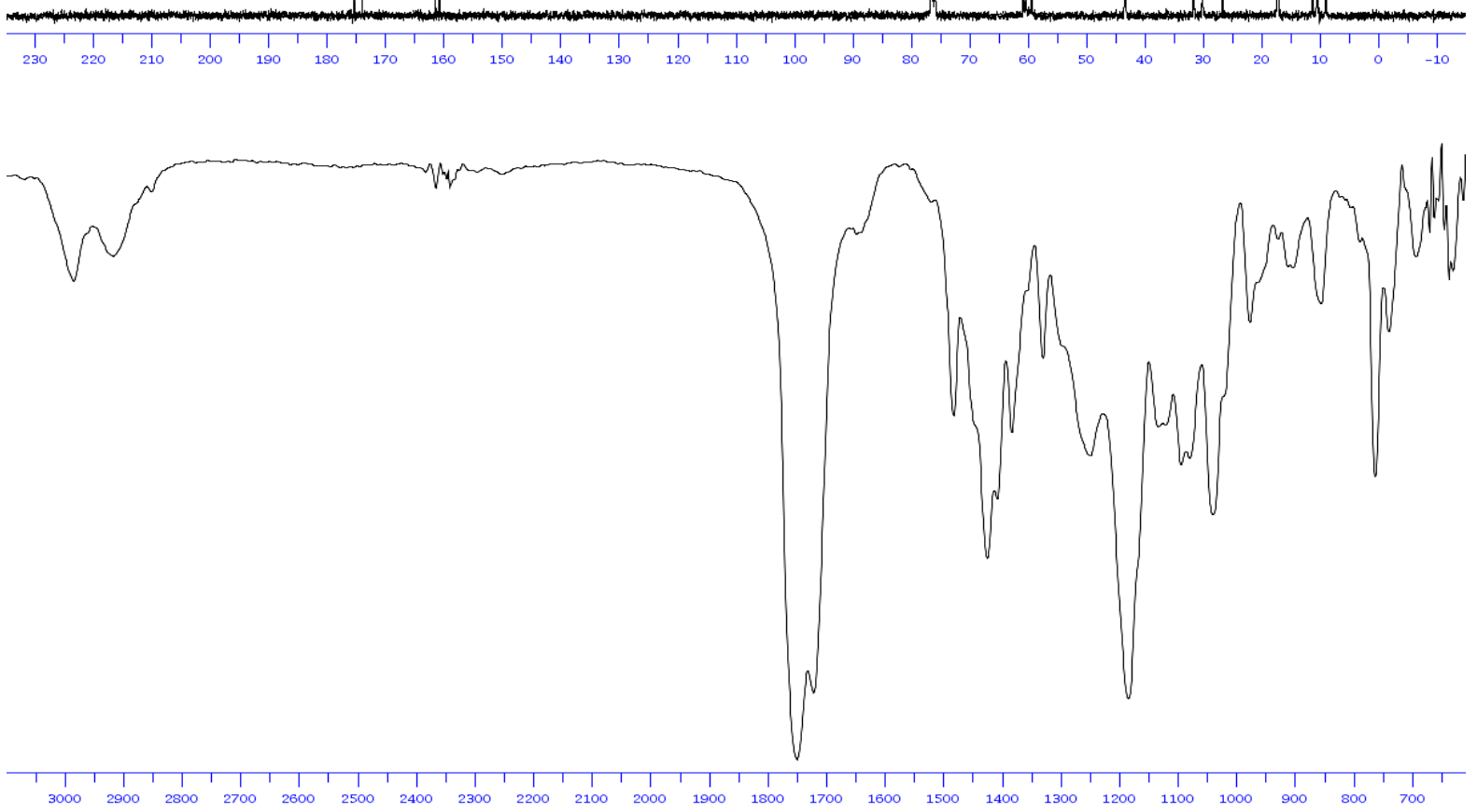
Solvent: $<\mathrm{CDCl} 3>$
Frequency. $400.13 \mathrm{MHz}$
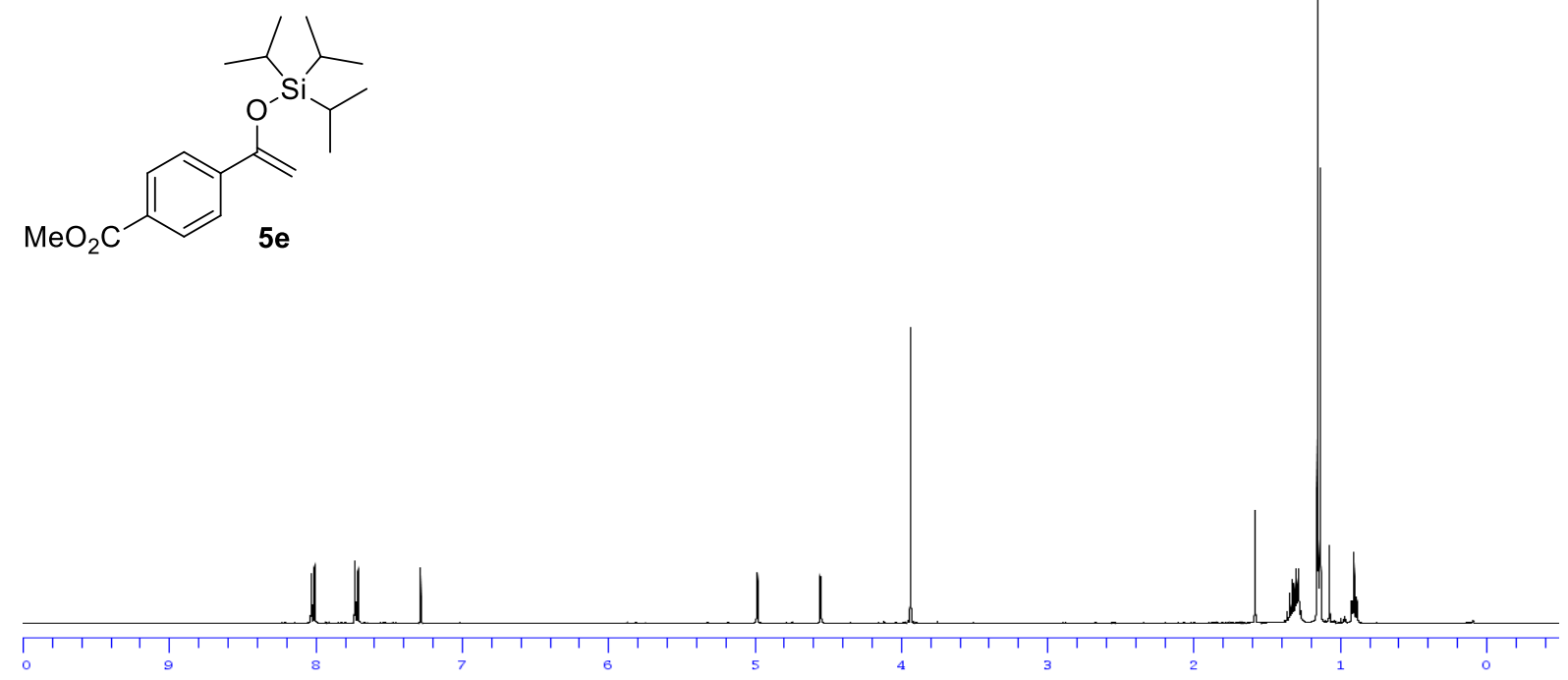

Solvent: $\angle C D C 13>$
Frequency: $100.612769 \mathrm{MHz}$
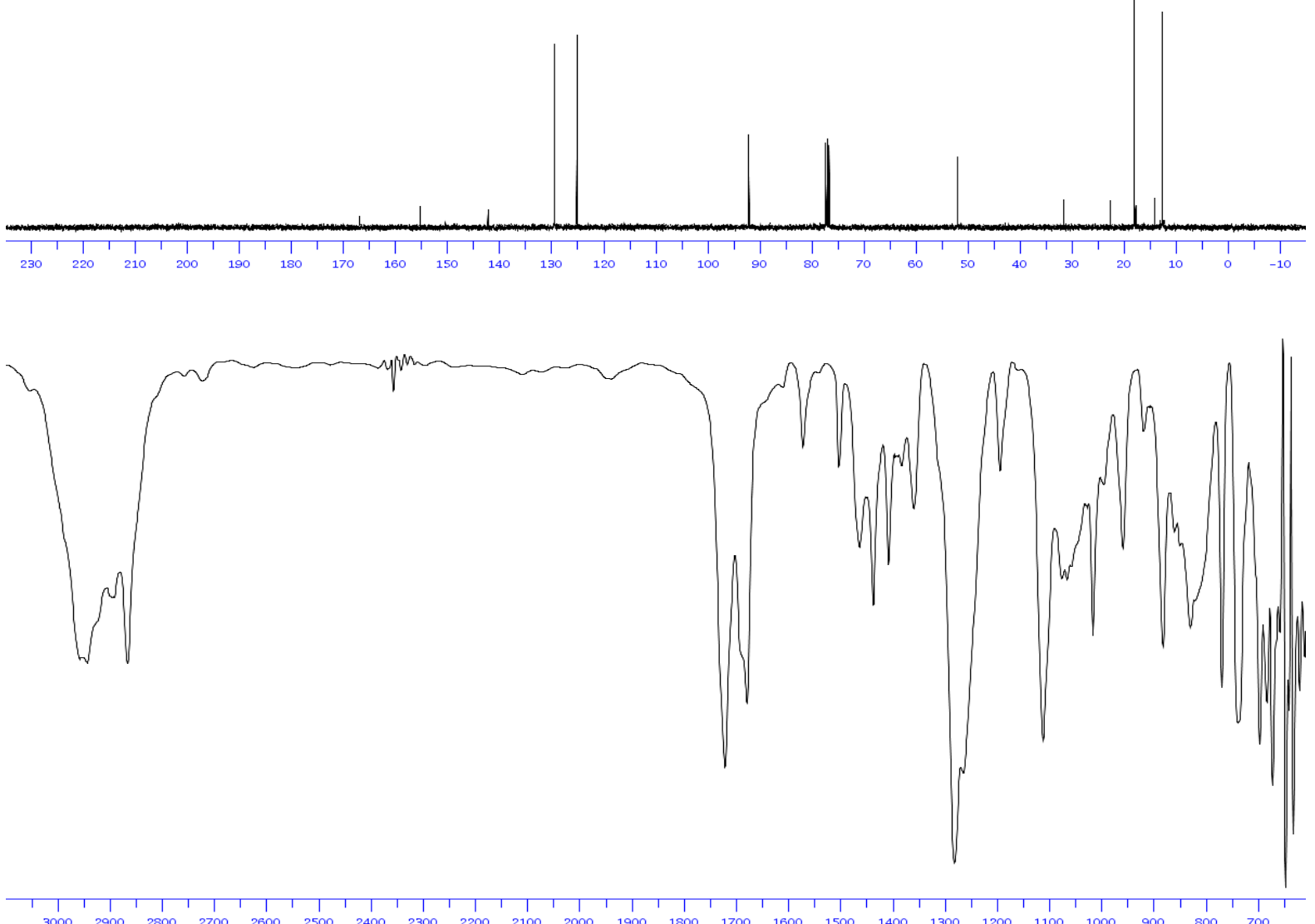

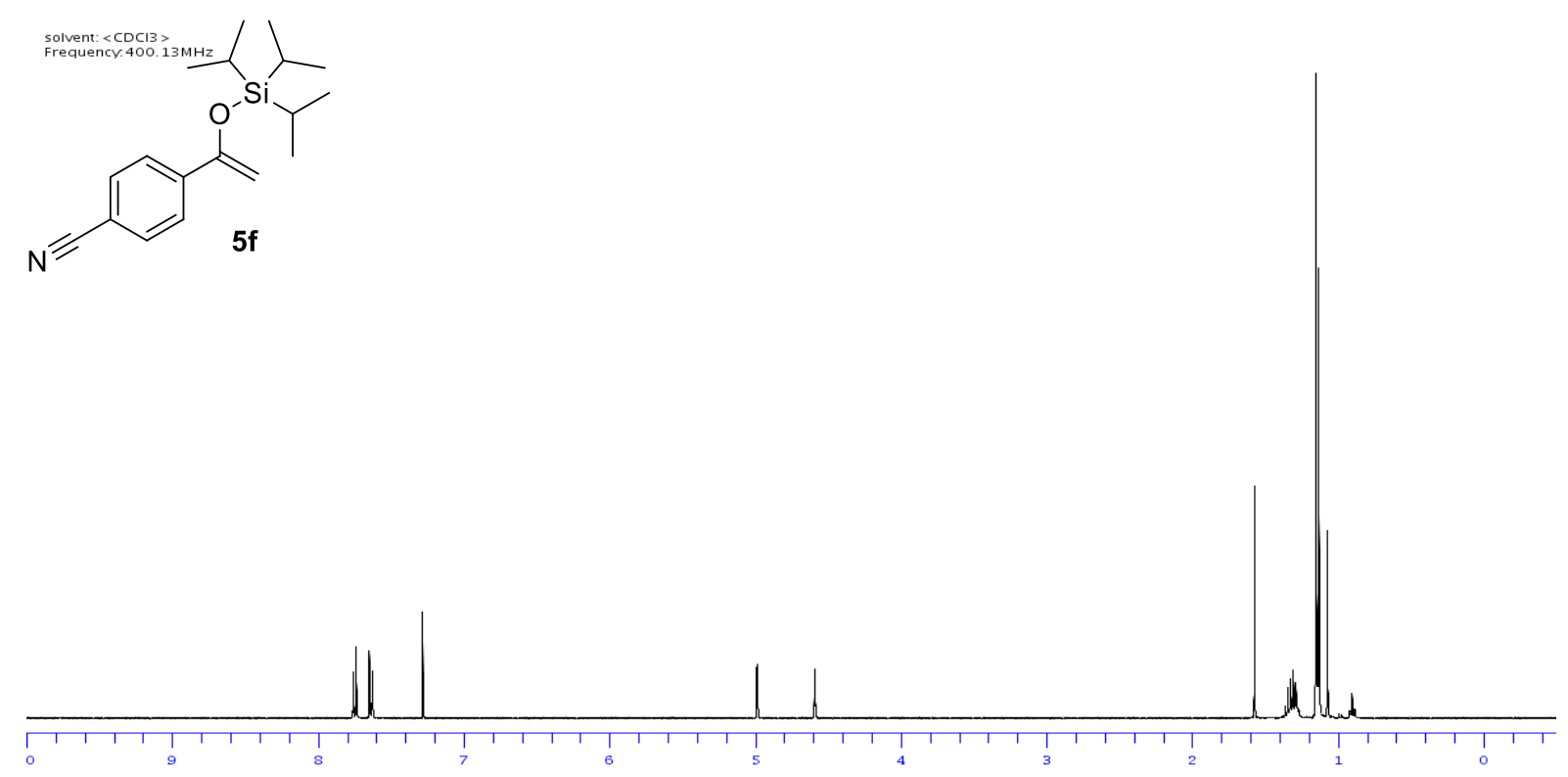

solvent: $<C D C 13>$
Frequency. 100. $112769 \mathrm{MHz}$
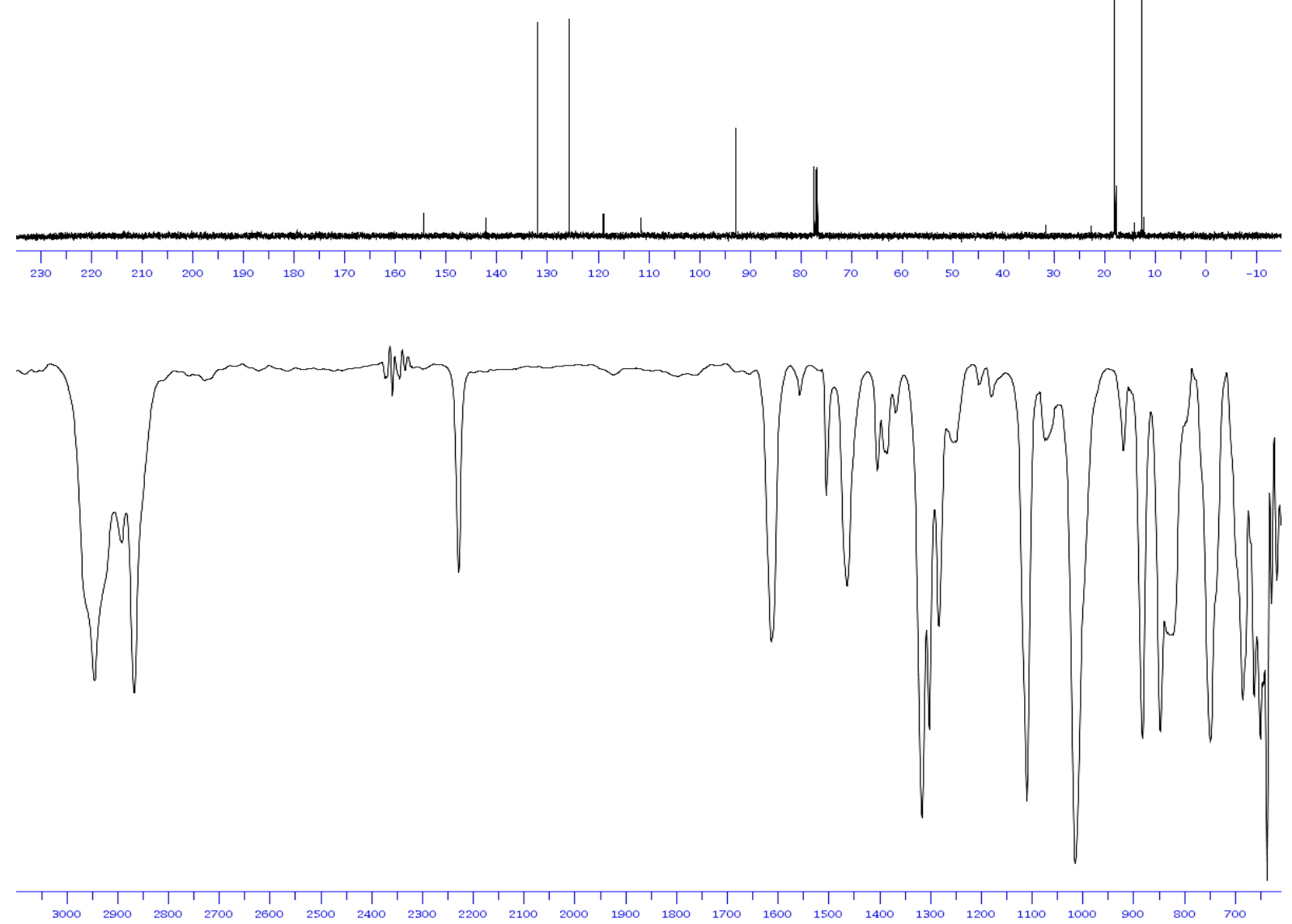
Solvent: $<C D C 13>$
Frequency: $400.13 \mathrm{MHz}$

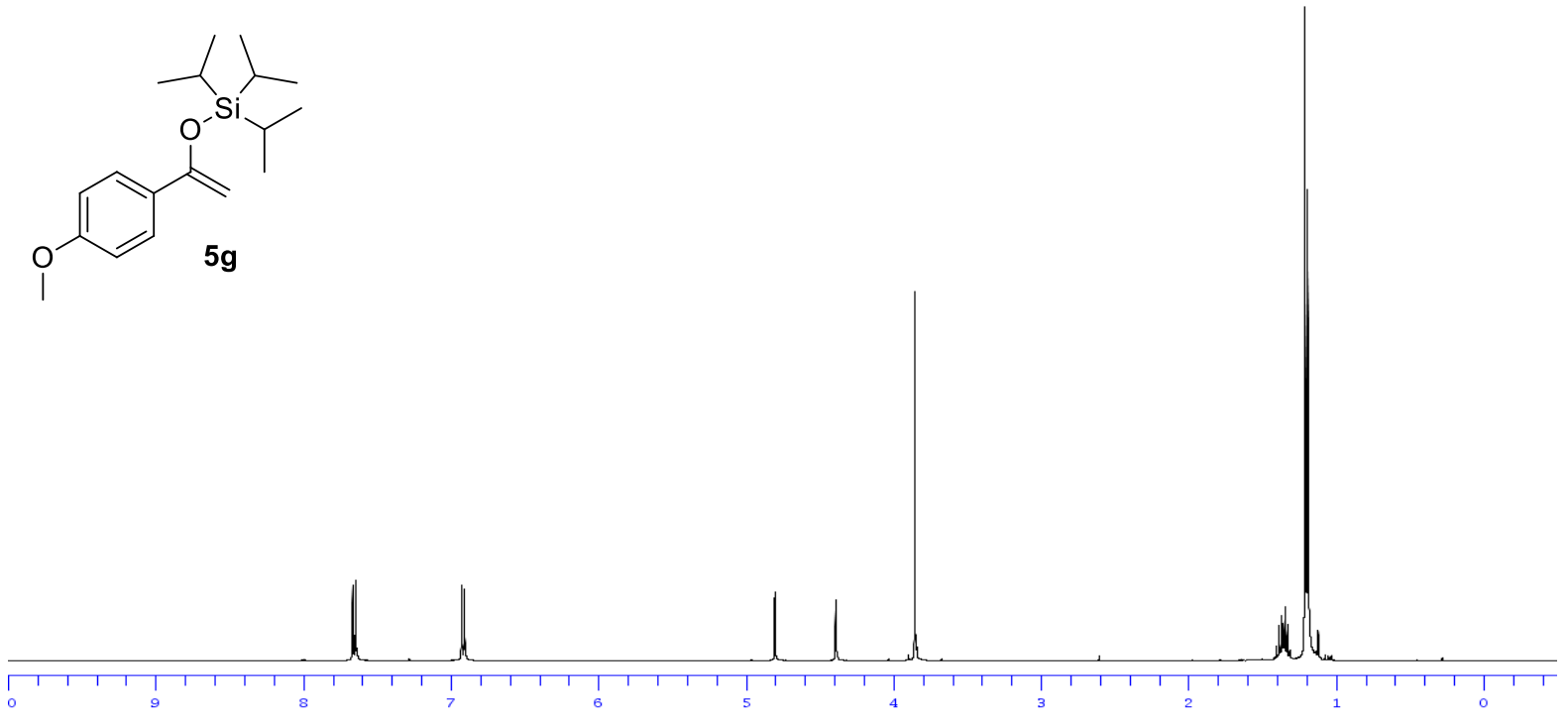

Solvent: $<C D C l 3>$
Frequency. 100. $12769 \mathrm{MHz}$

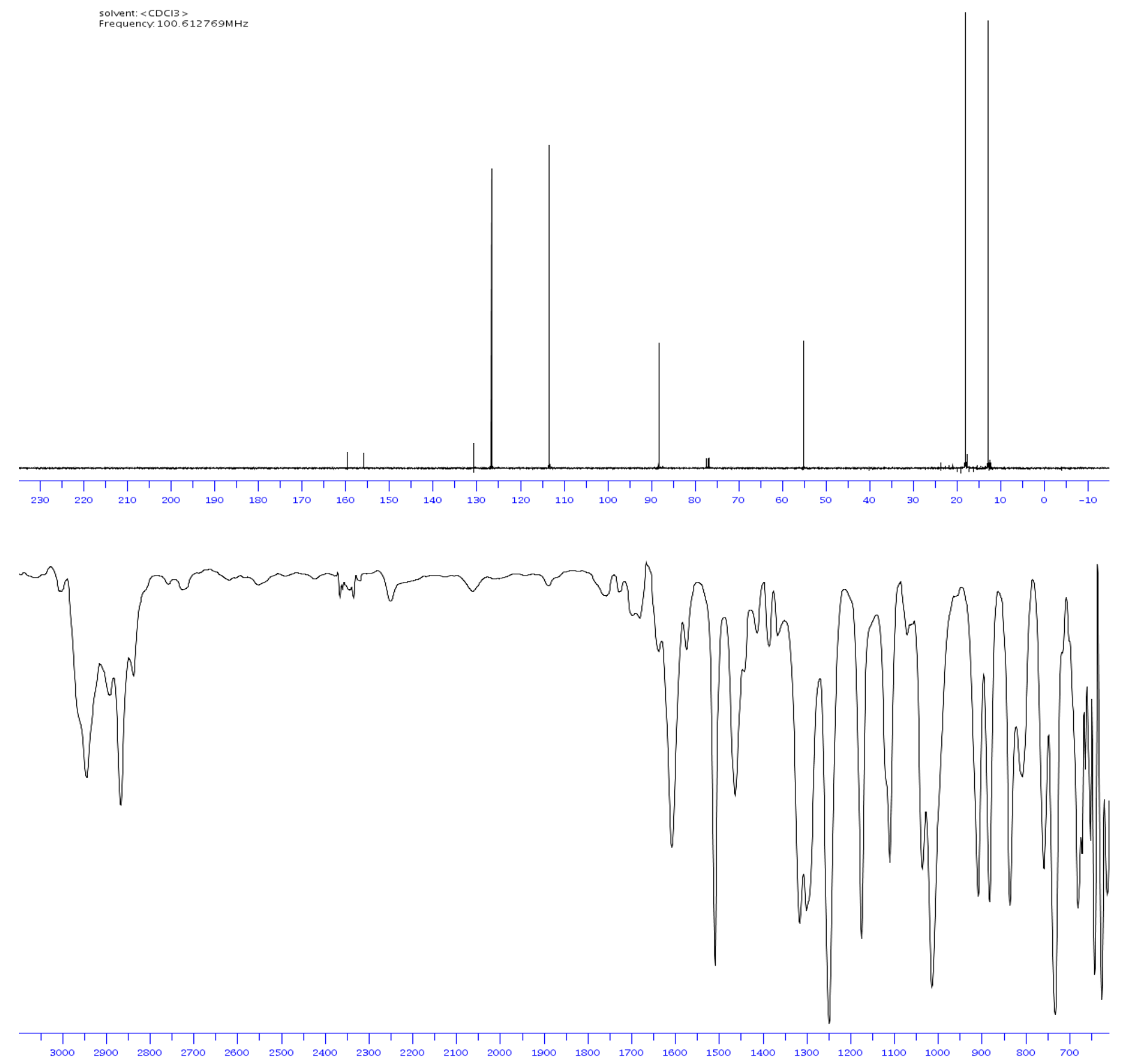


solvent: $<C D C 13>$
Frequency $400.13 \mathrm{MH}$
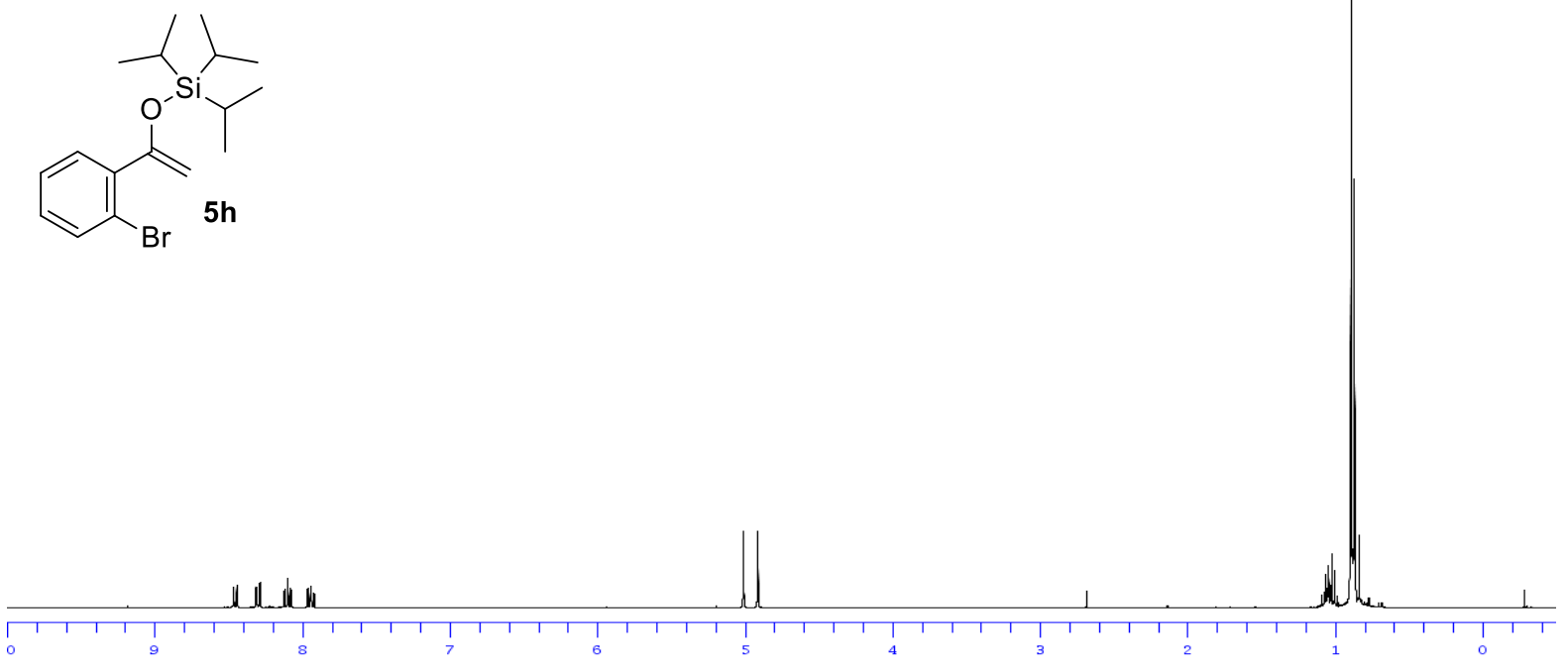

solvent: $<C D C 13>$
Frequency. 100.612769 MHz
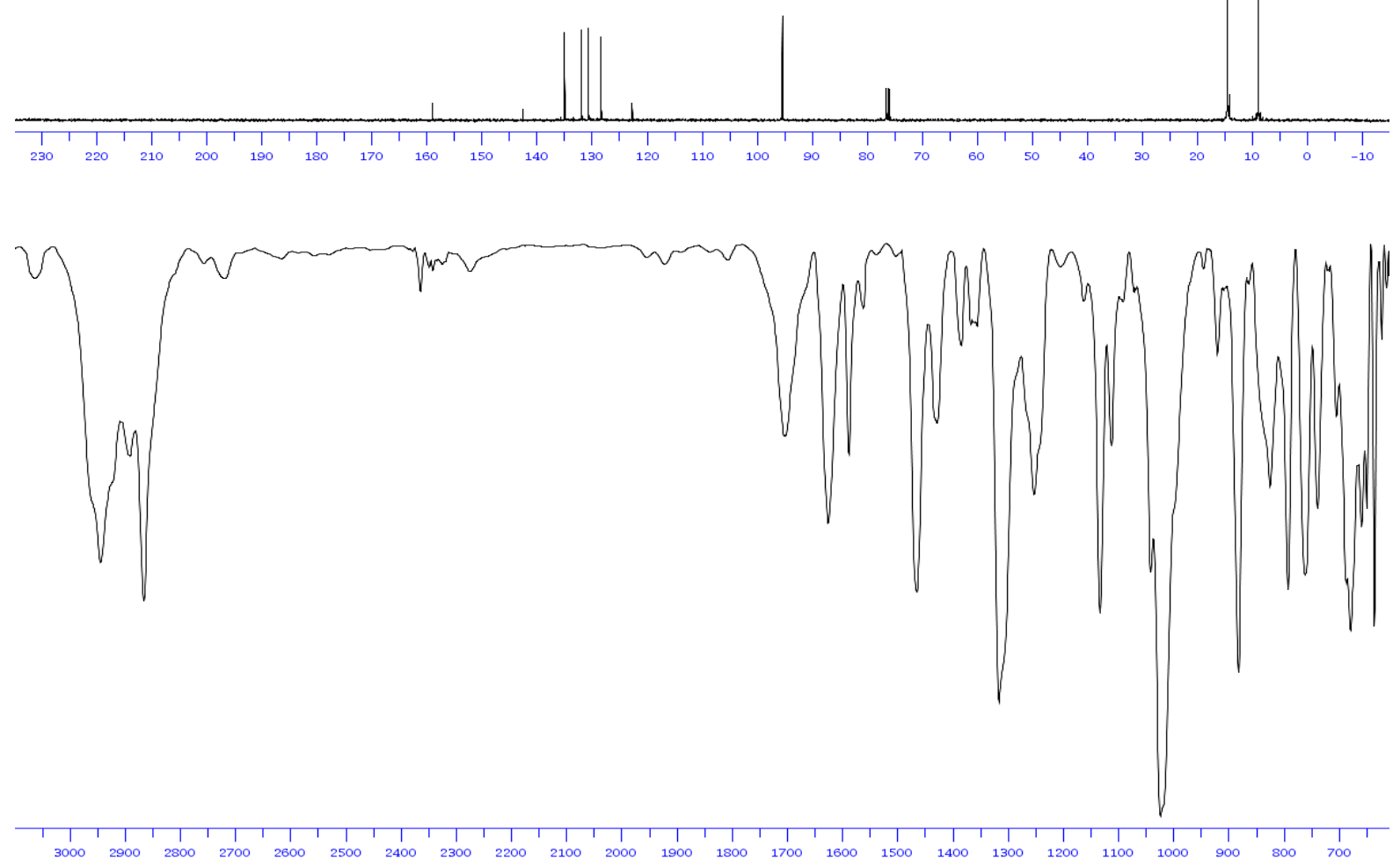
Solvent: $\langle\mathrm{CDCl} 3\rangle$
Frequency: $400.13 \mathrm{MHz}$

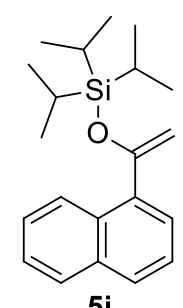

$5 i$

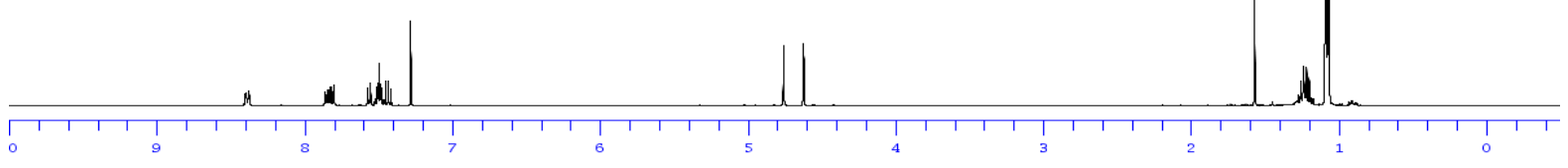

Solvent: $<\mathrm{CDCl3}>$
Frequency. 100.612769MHz
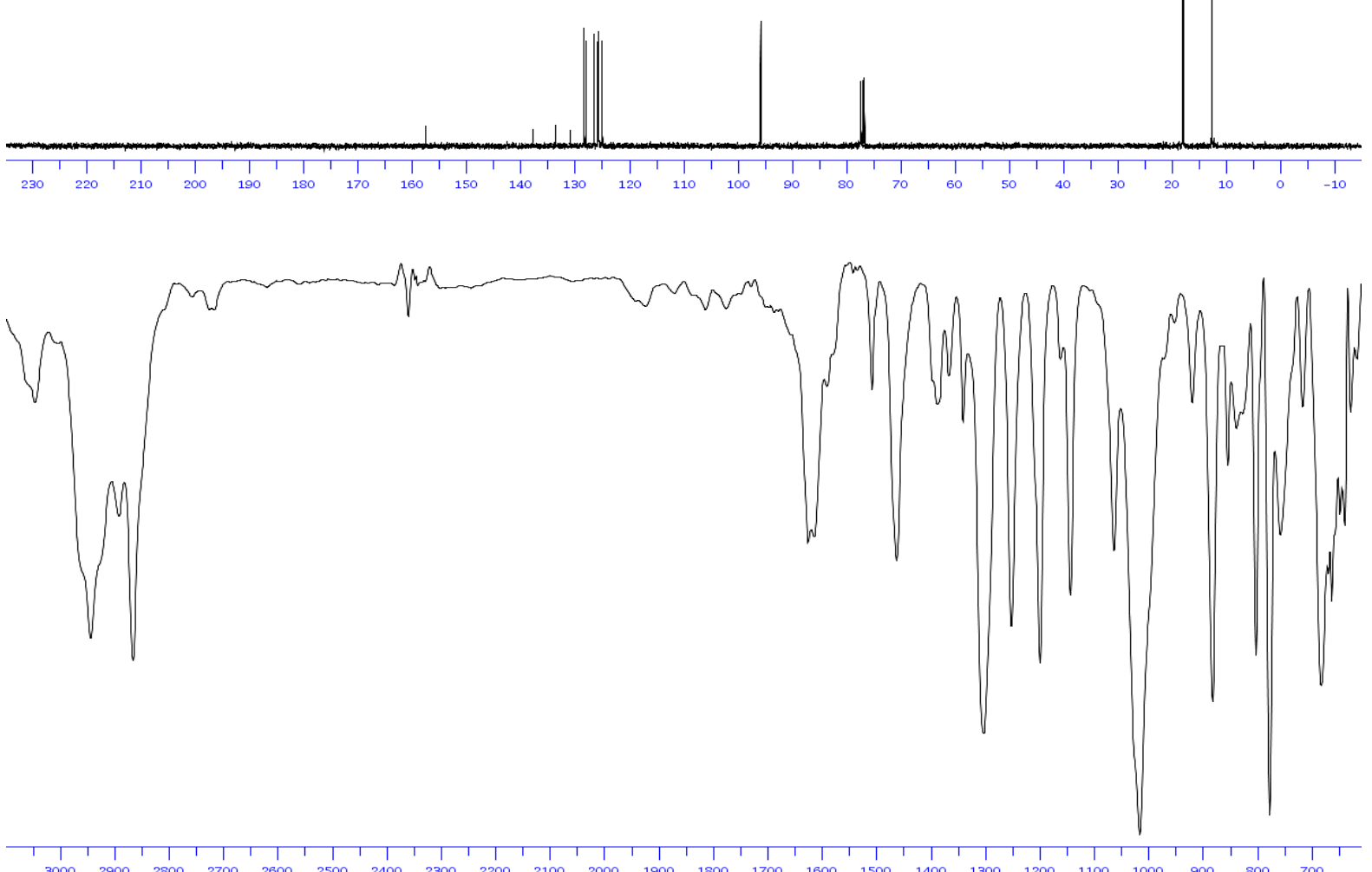
solvent: $<\mathrm{CDCl} 3>$
Frequency $400.13 \mathrm{MH}$

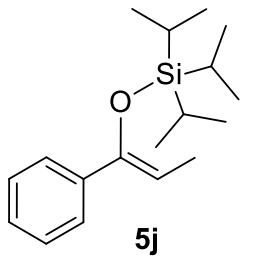

uh

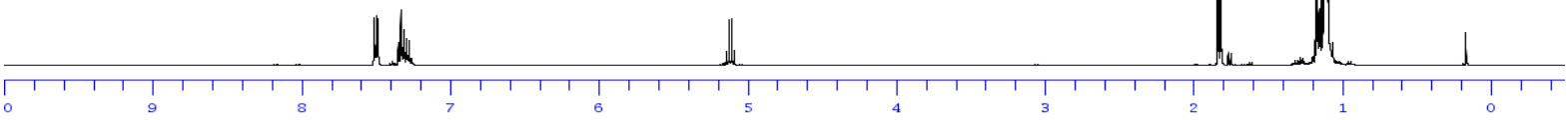

solvent: $<C D C 13>$
Frequency. 100.612769 MHz
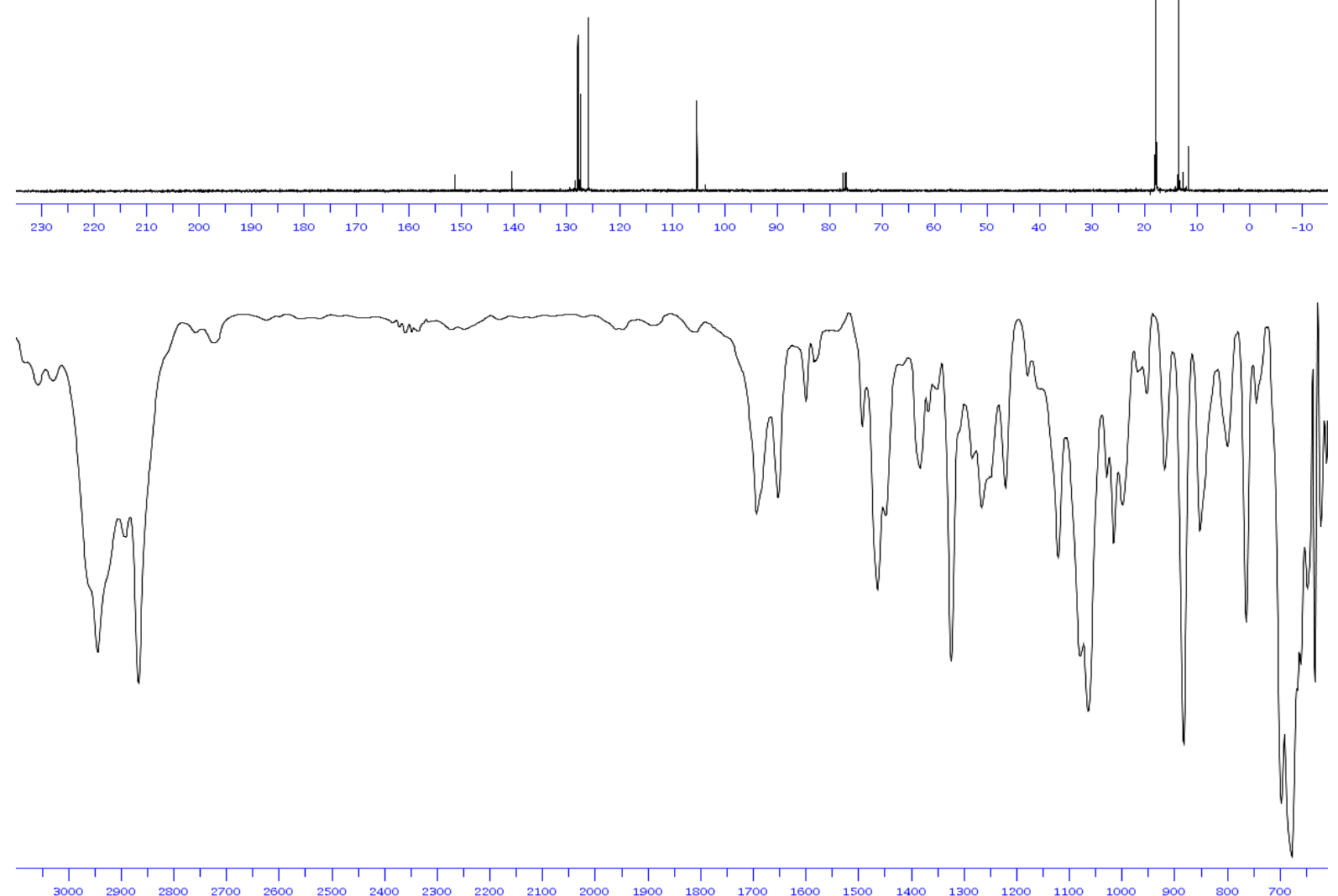
Solvent: $<\mathrm{CDCl} 3>$
Frequency $400.13 \mathrm{MH}$
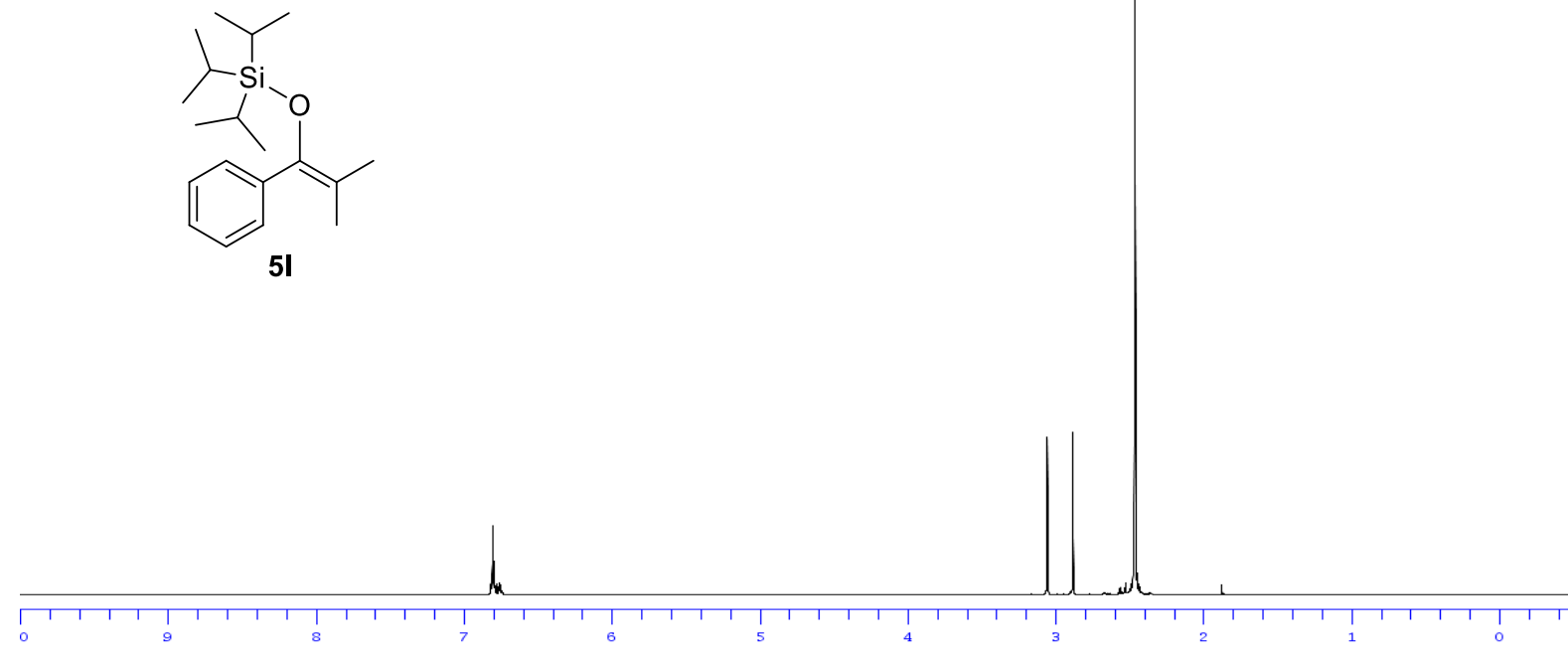

Solvent: $<\mathrm{CDCl} 3>$
Frequency. 100.612769M Hz
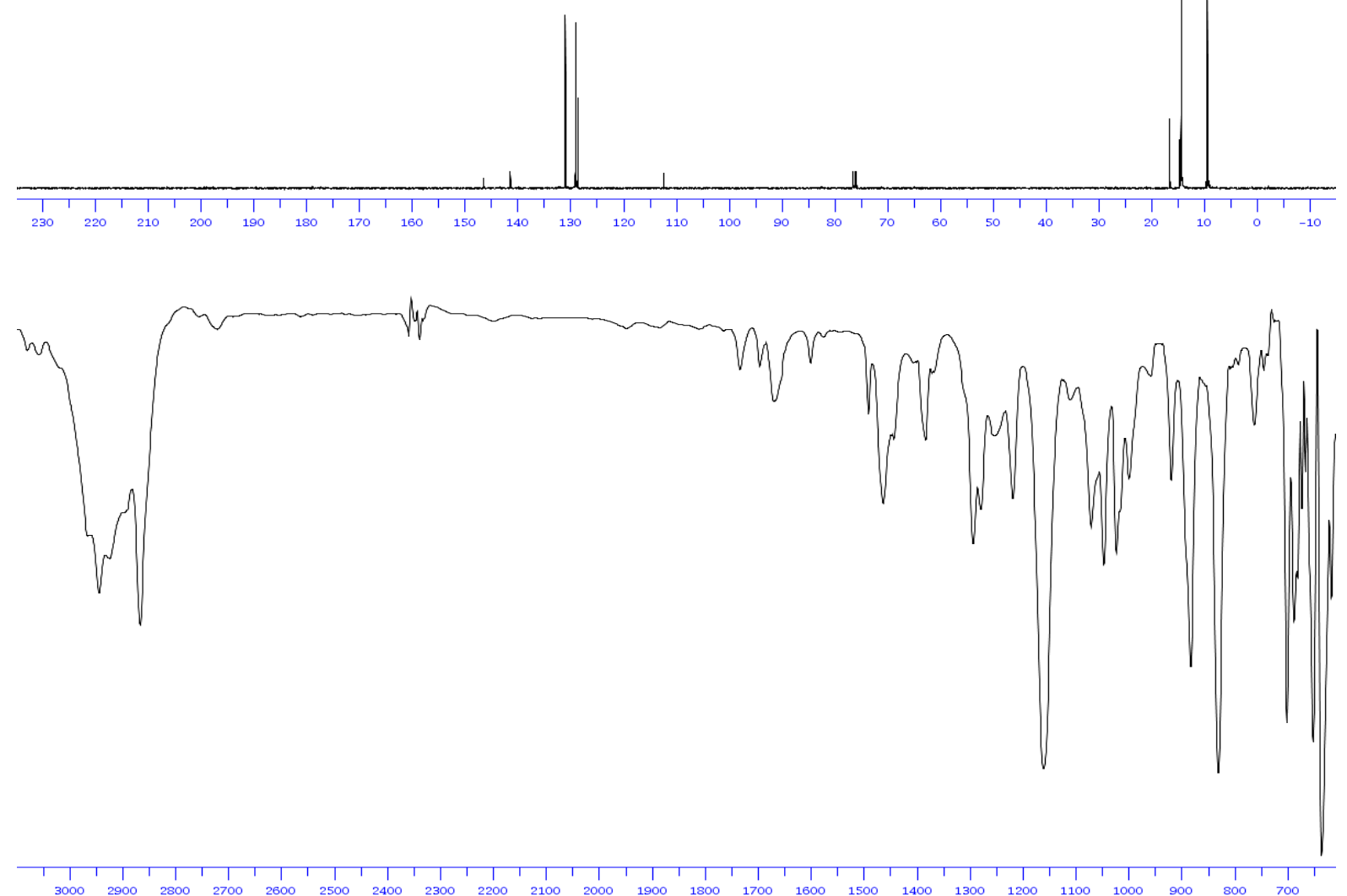
solvent: $<C D C 13>$
Frequency: $400.13 \mathrm{MHz}$

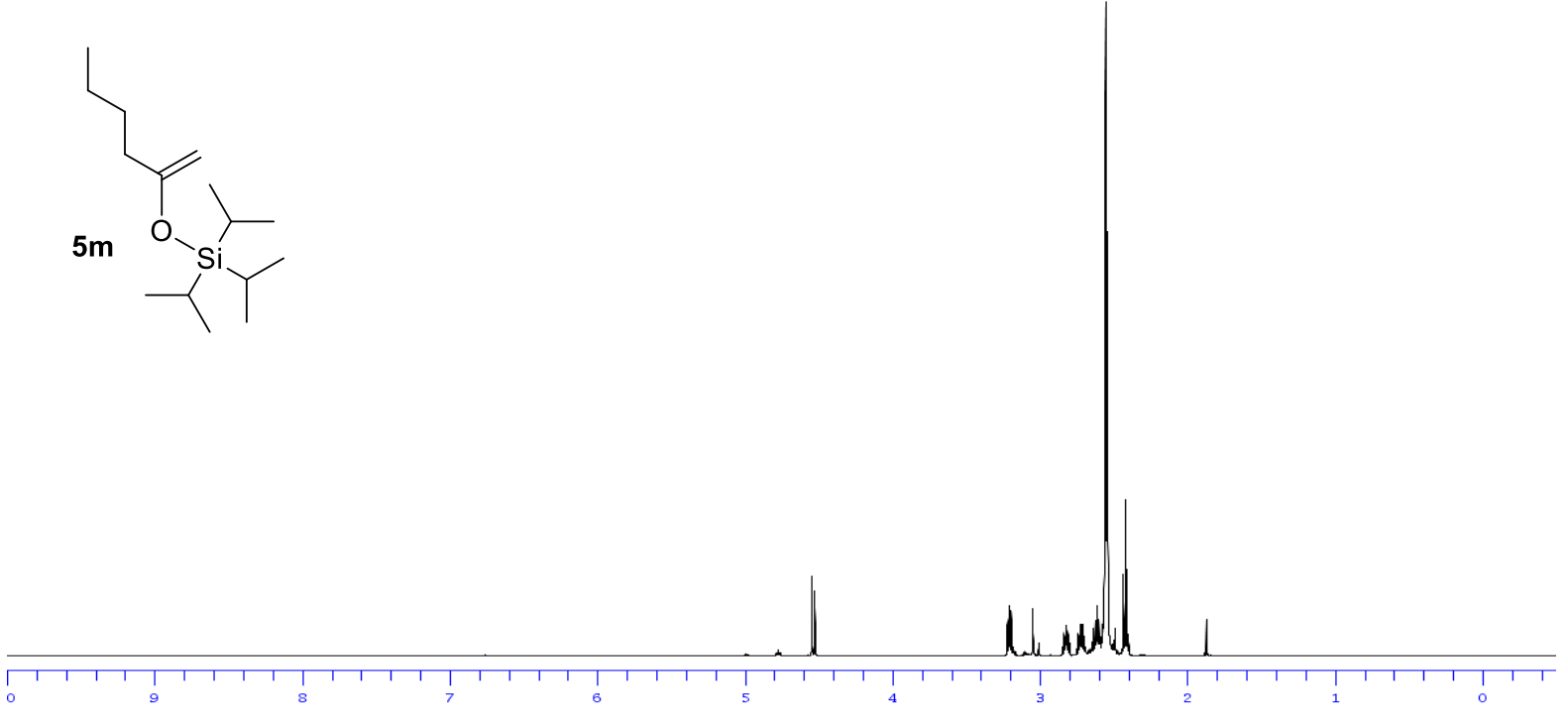

solvent: $<C D C I 3>$
Frequency. 100.612769M Hz
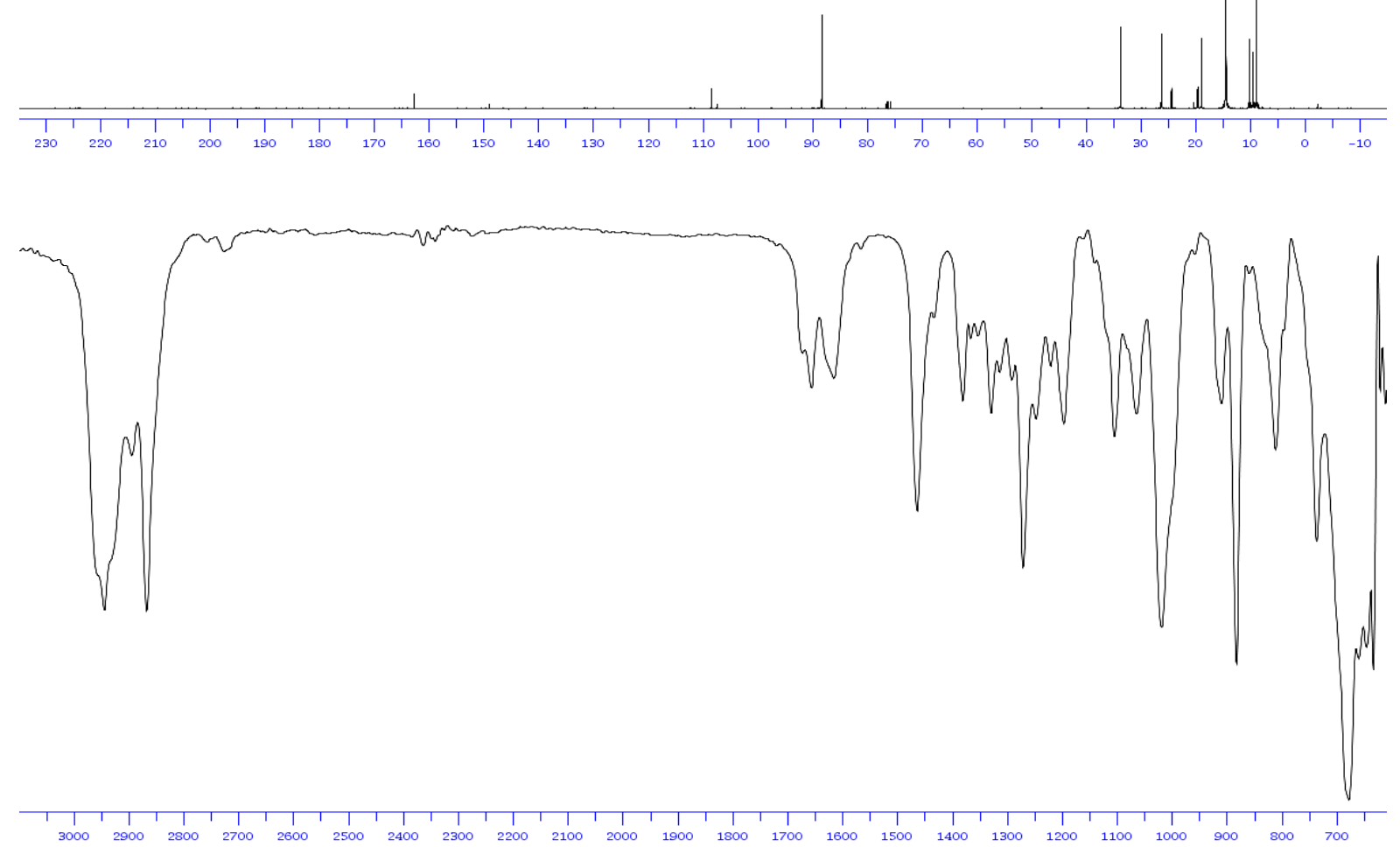

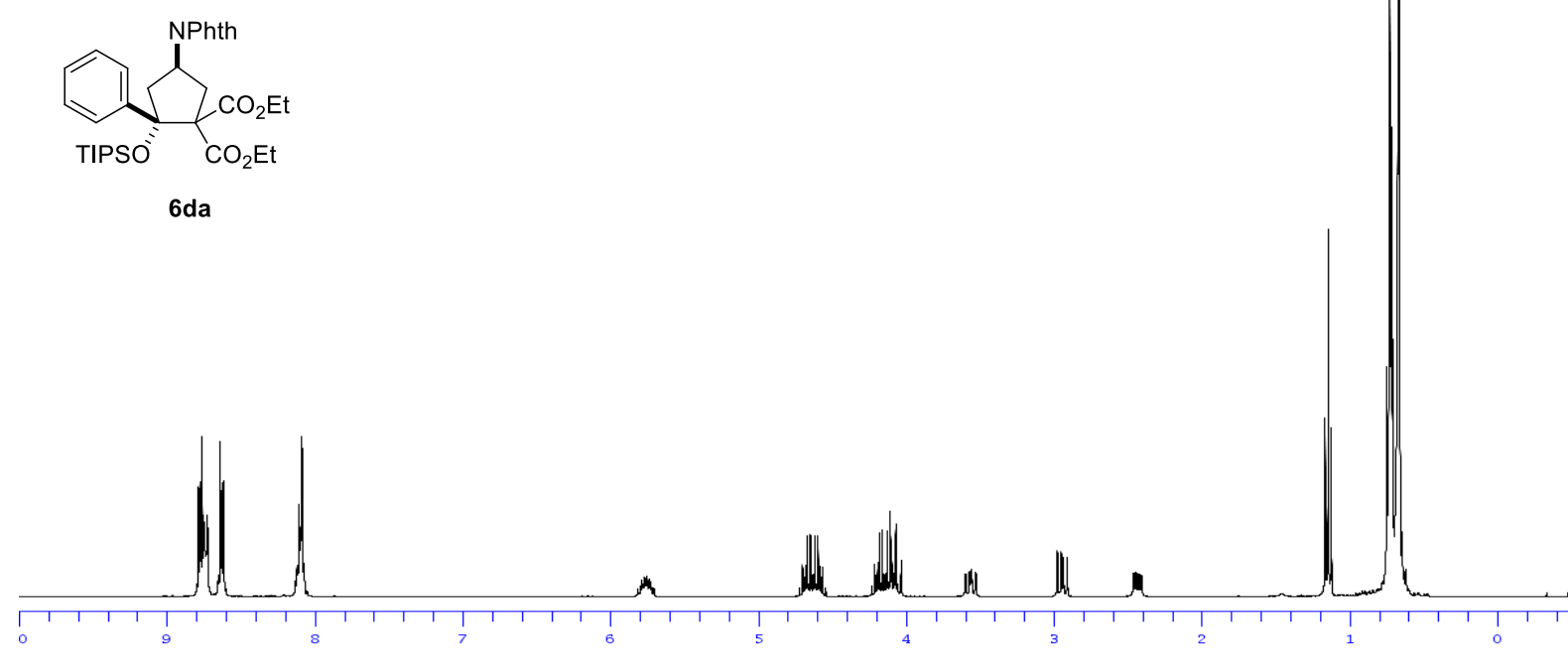

Solvent: $<$ CDCI3 $>$
Frequency. $100.612769 \mathrm{MHz}$
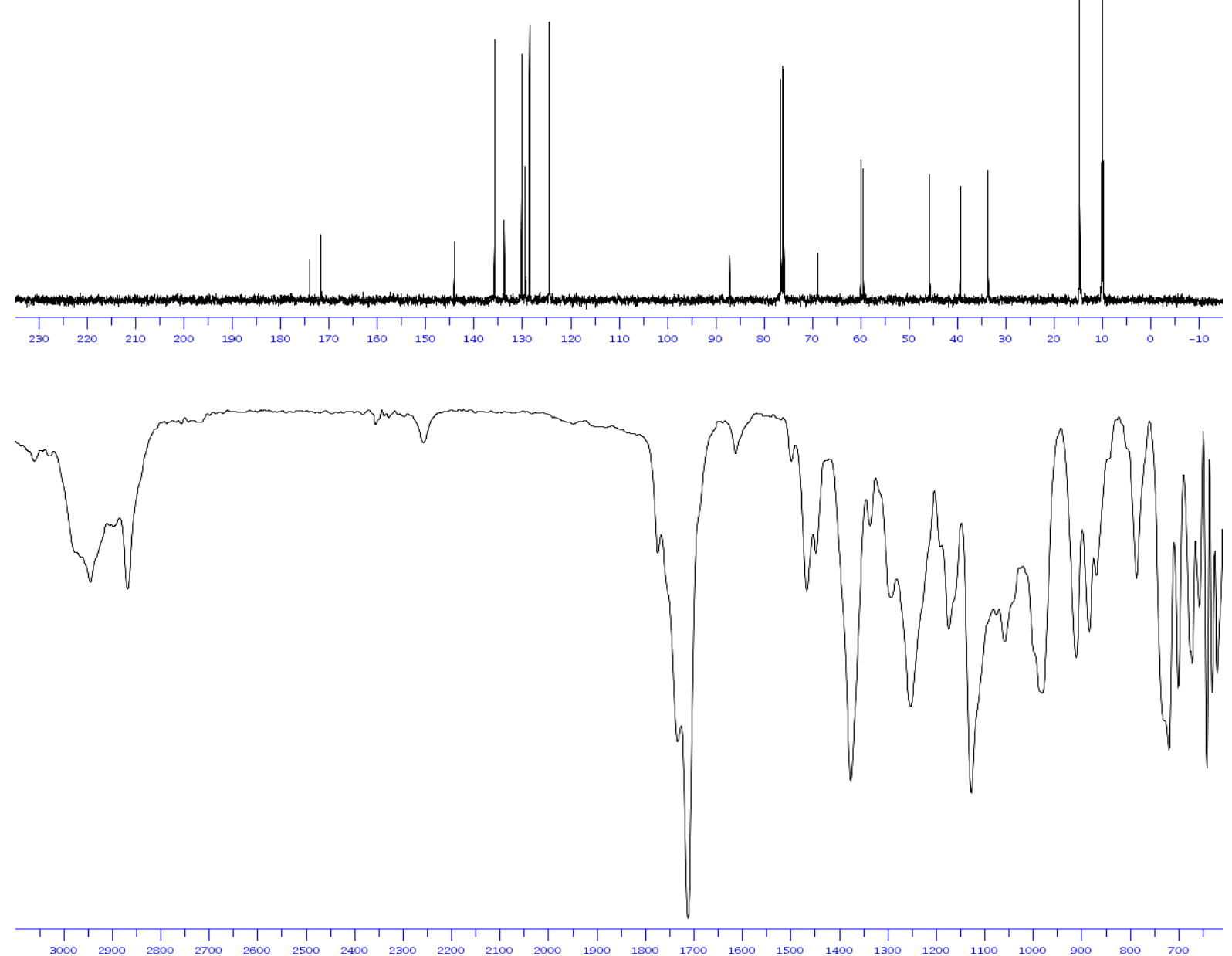
Solvent: $\langle\mathrm{CDCl} 3\rangle$
Frequency: $400.13 \mathrm{MHz}$

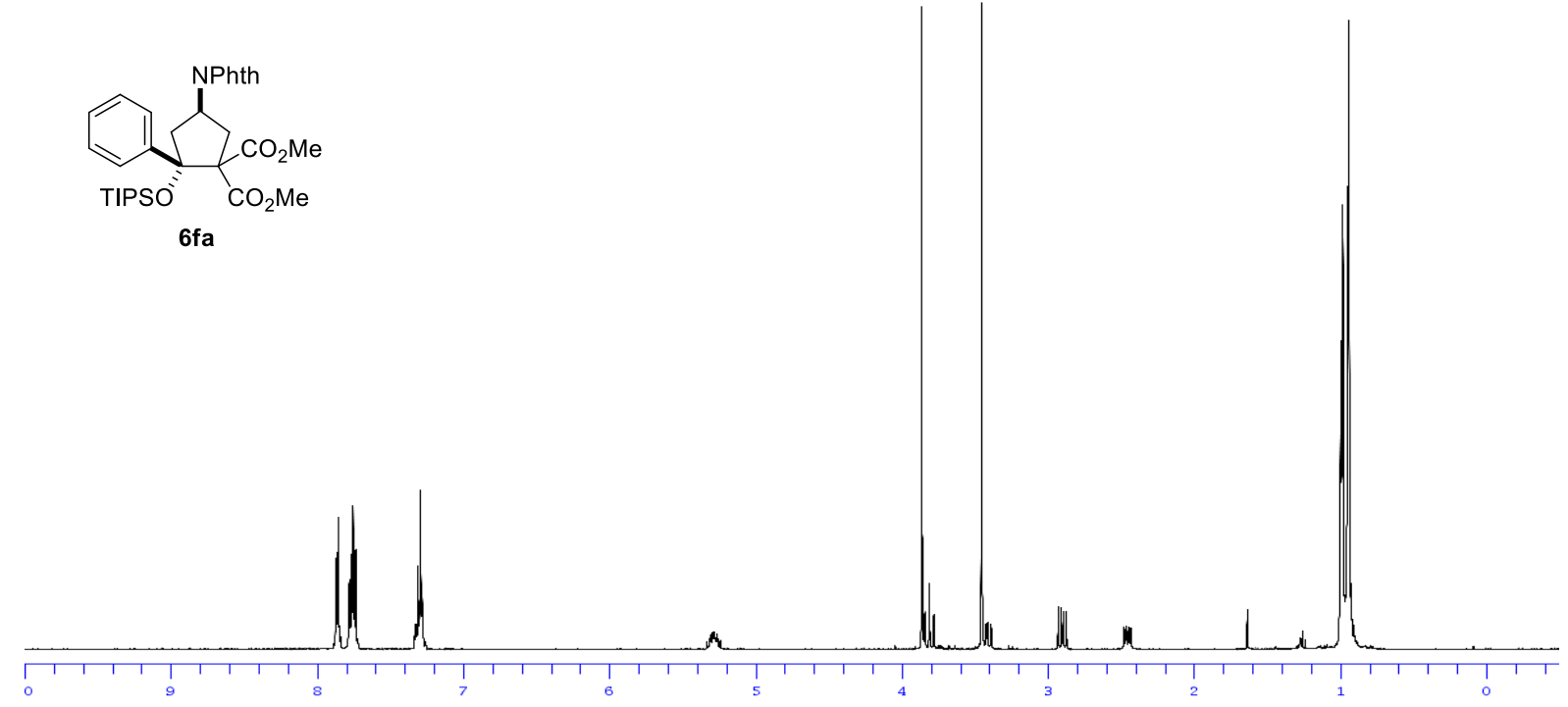

solvent: $<\mathrm{CDCl}>$
Frequency. $100.612769 \mathrm{MHz}$
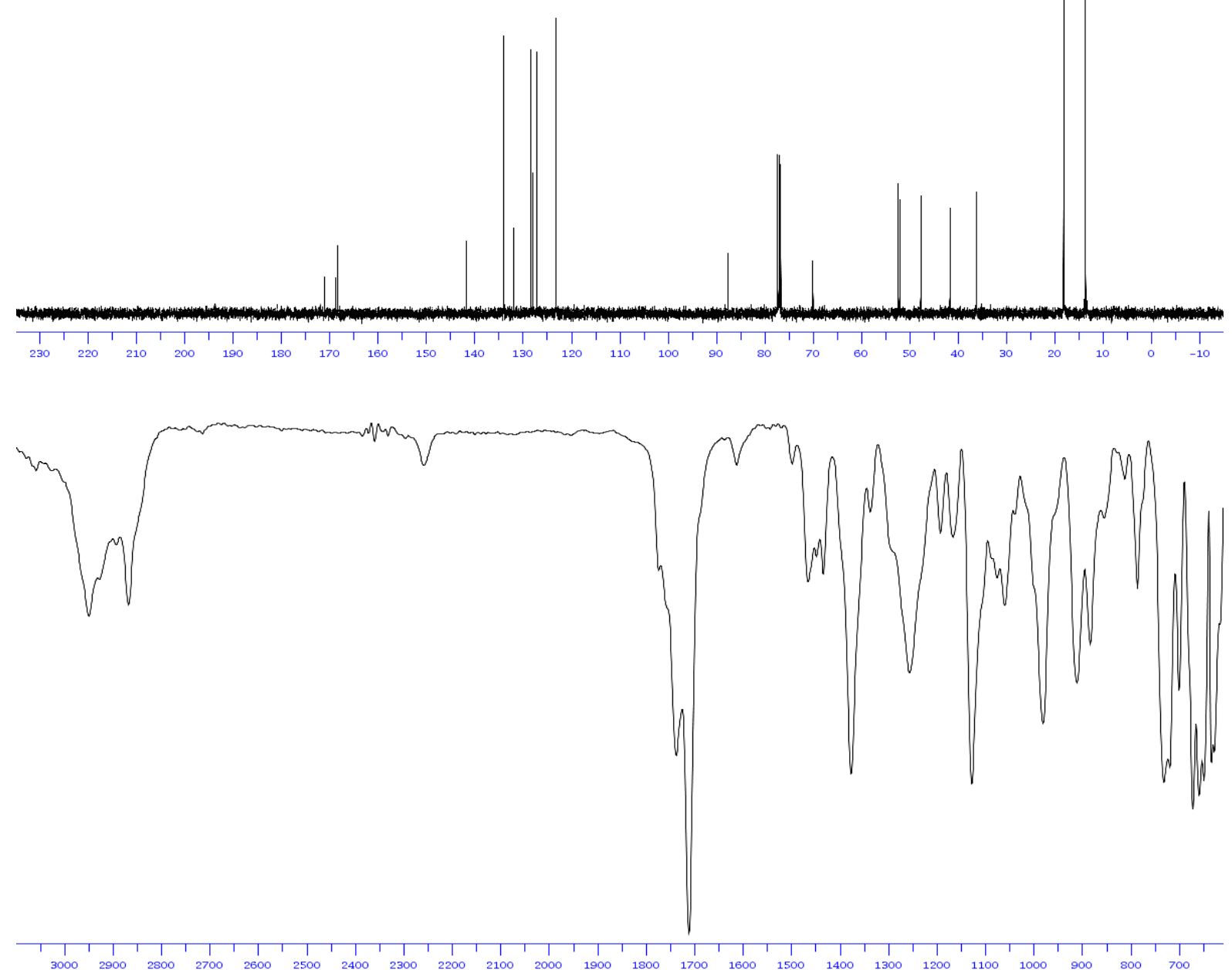
Solvent: $\langle\mathrm{CDCl} 3\rangle$
Frequency: $400.13 \mathrm{MHz}$

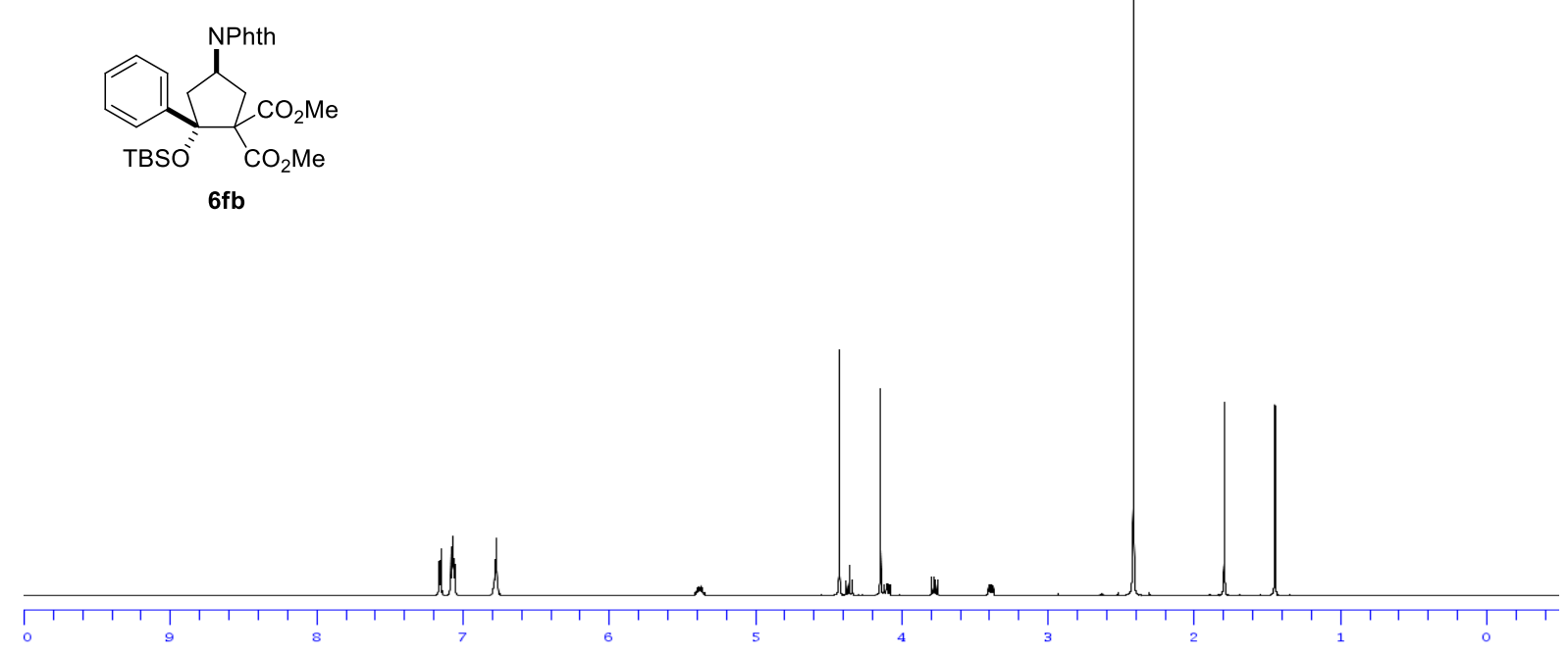

Solvent: $<\mathrm{CDCl}>$
Frequency: $100.612769 \mathrm{MHz}$
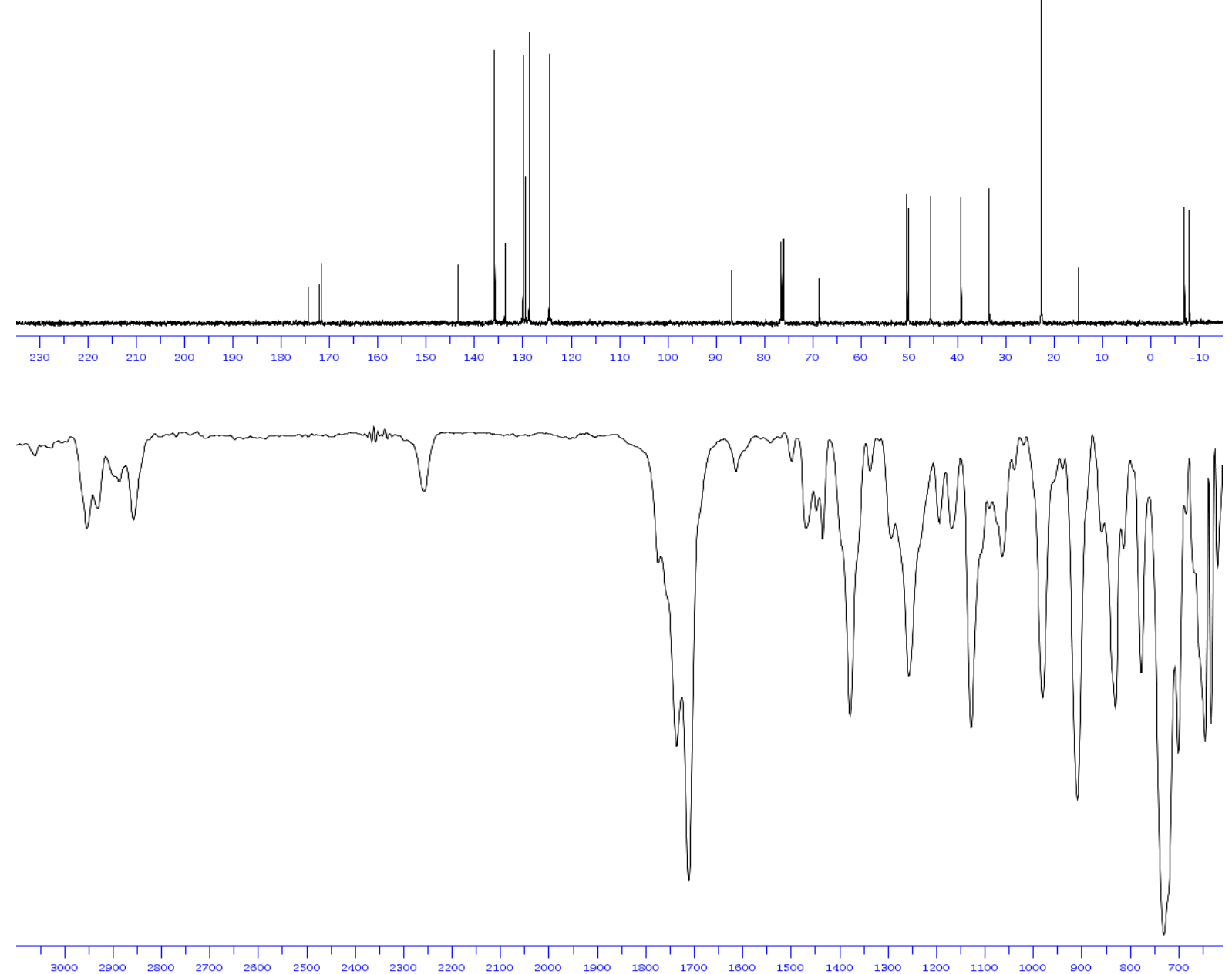


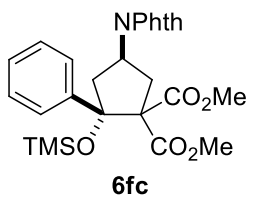

do

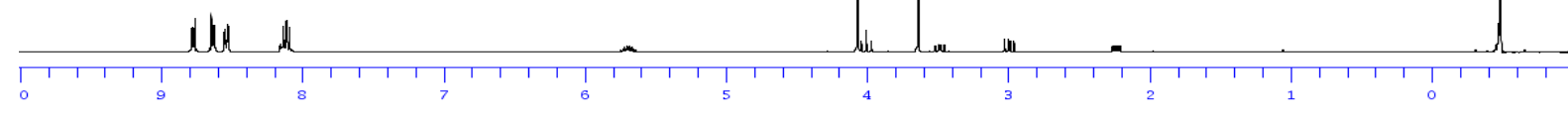

solvent: $<$ CDCI3 >
FrequencY: $100.512769 \mathrm{MHz}$
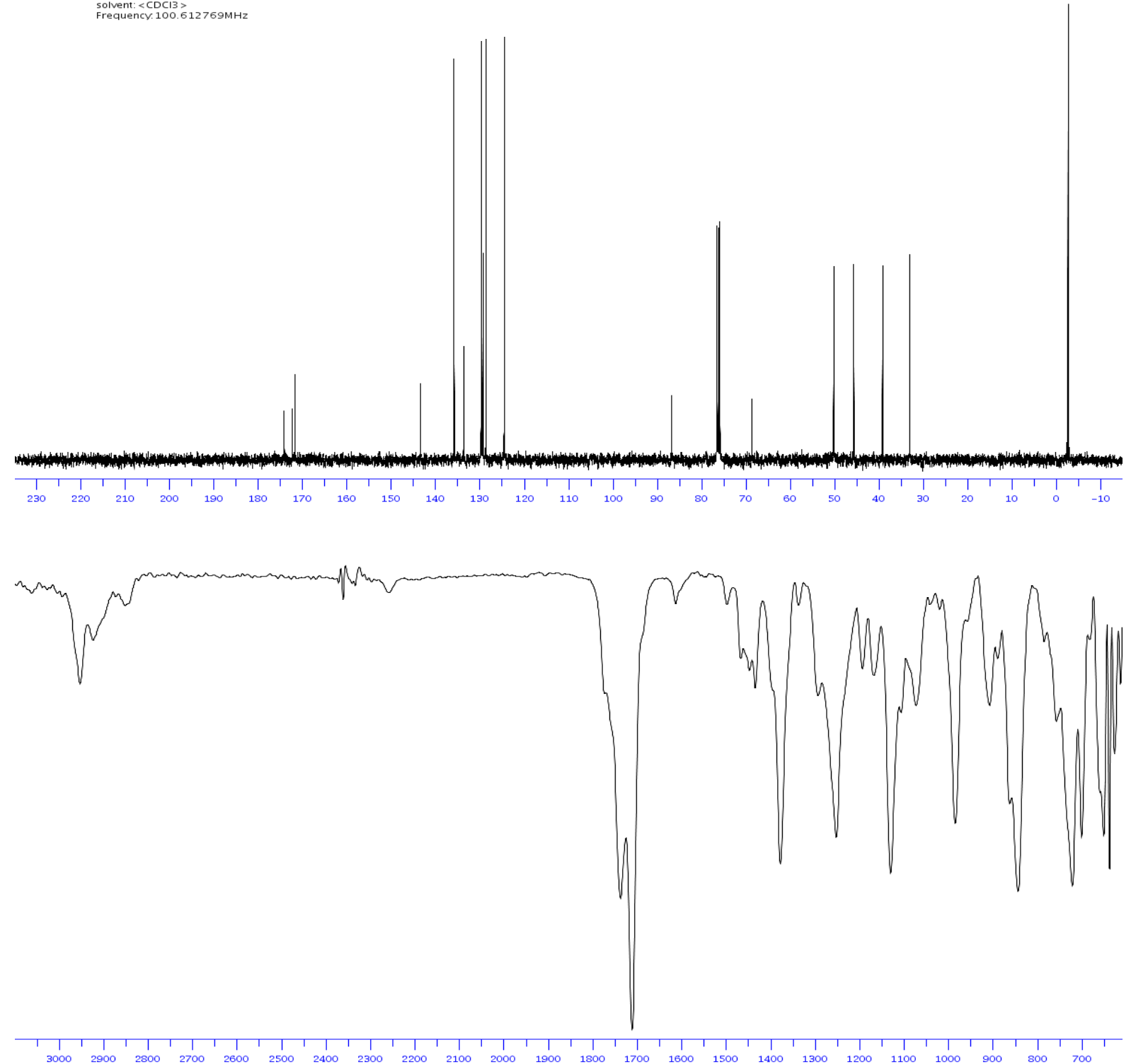
solvent: $\langle\mathrm{CDCl} 3\rangle$
Frequency: $400.13 \mathrm{MHz}$
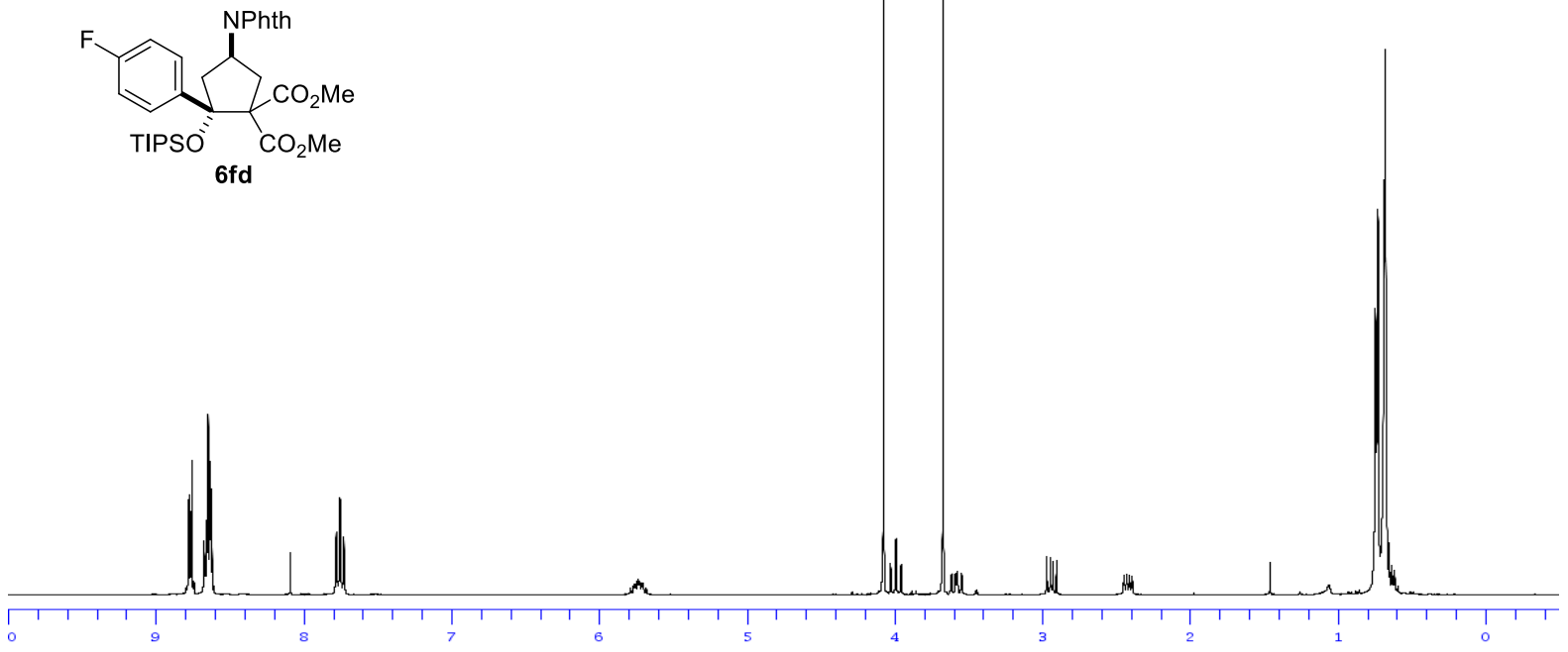

Solvent: $<\mathrm{CDCl3}>$
Frequency: $100.612769 \mathrm{MHz}$
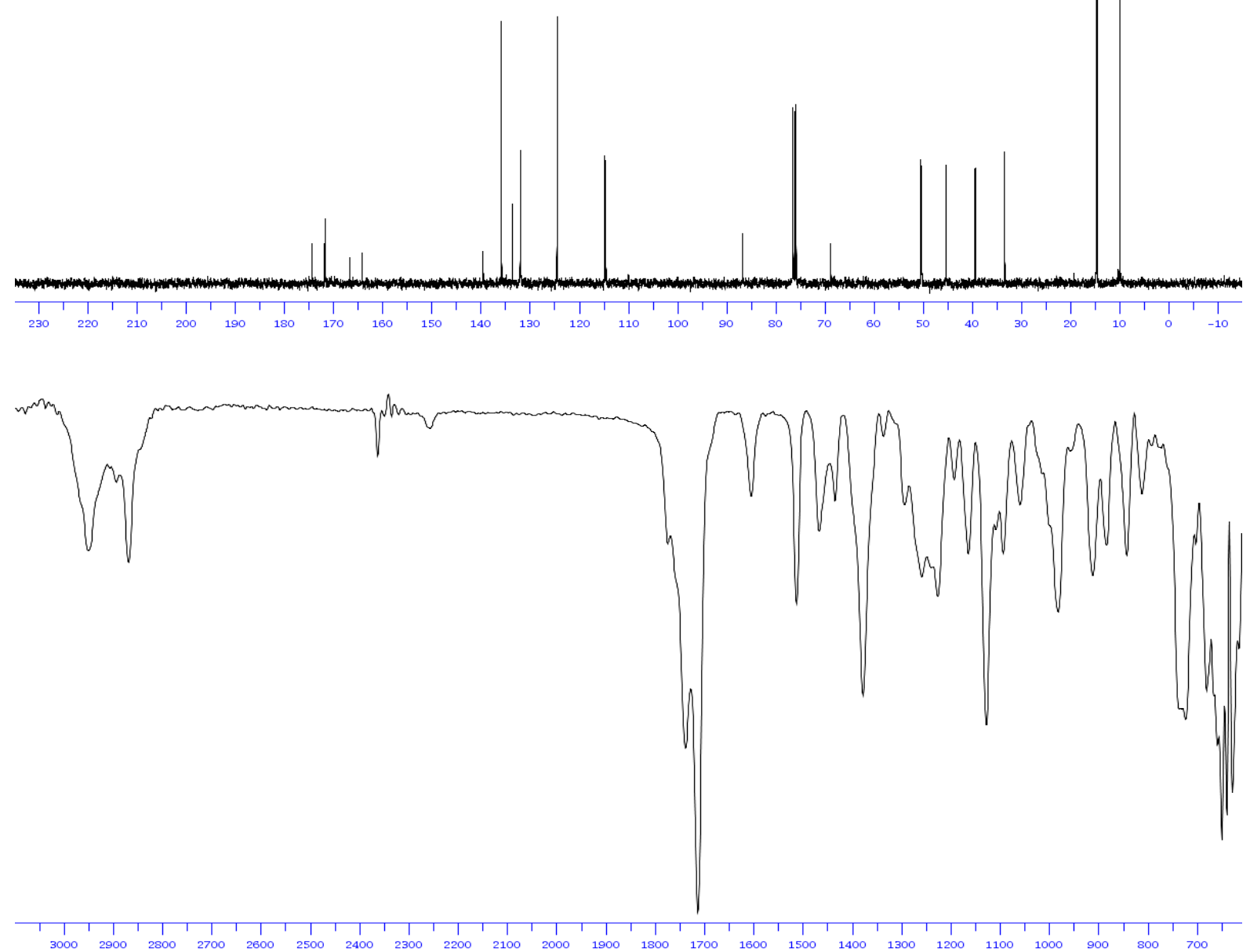
solvent: $<\mathrm{CDCl} 3>$
Frequency $400.13 \mathrm{MHz}$
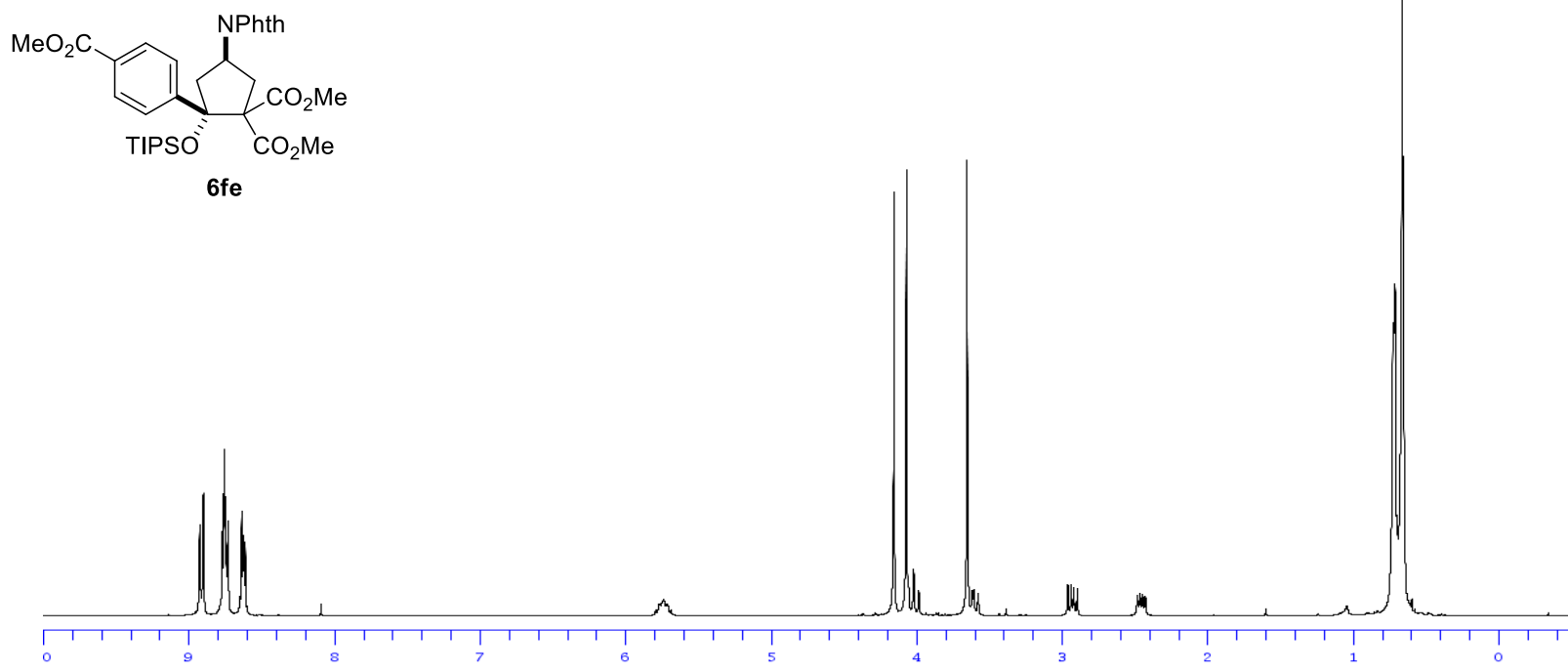

Solvent: $<\mathrm{CDCl3}>$
Frequency: $100.612769 \mathrm{MHz}$
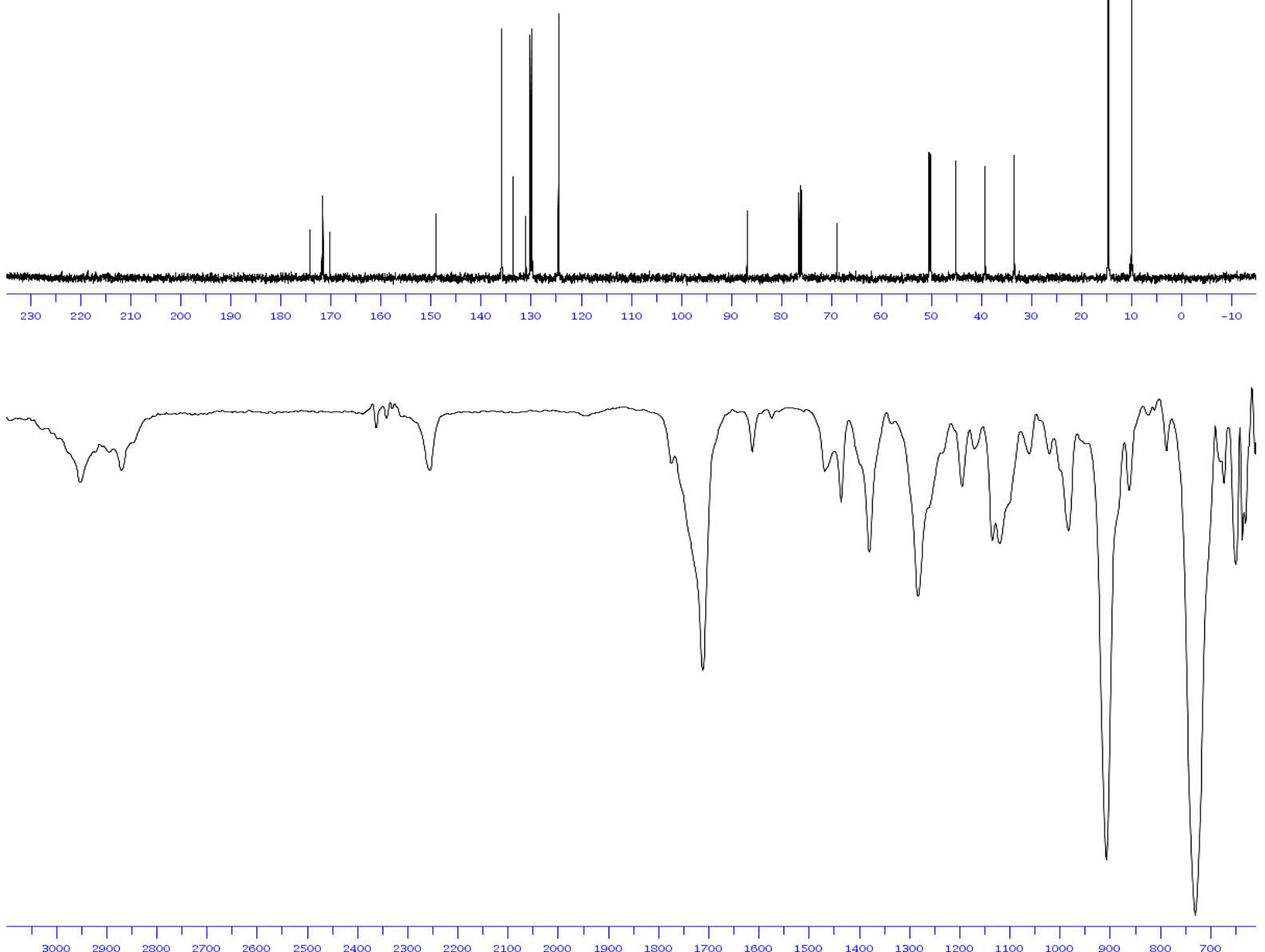
solvent: $\angle \mathrm{CDCl} 3>$
Frequency: $400.13 \mathrm{MHz}$
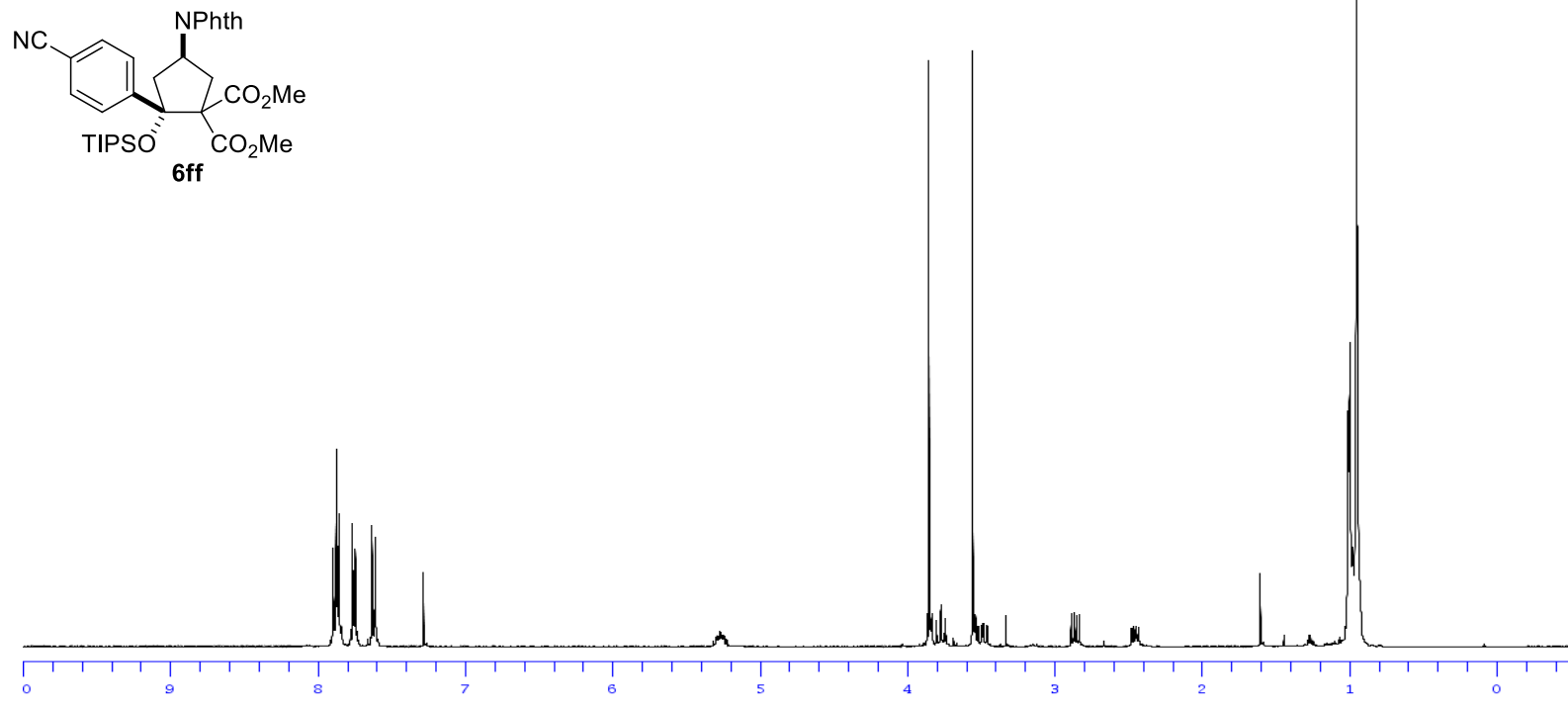

solvent: $<\mathrm{CDCl} 3>$
Frequency: $100.612769 \mathrm{MHz}$
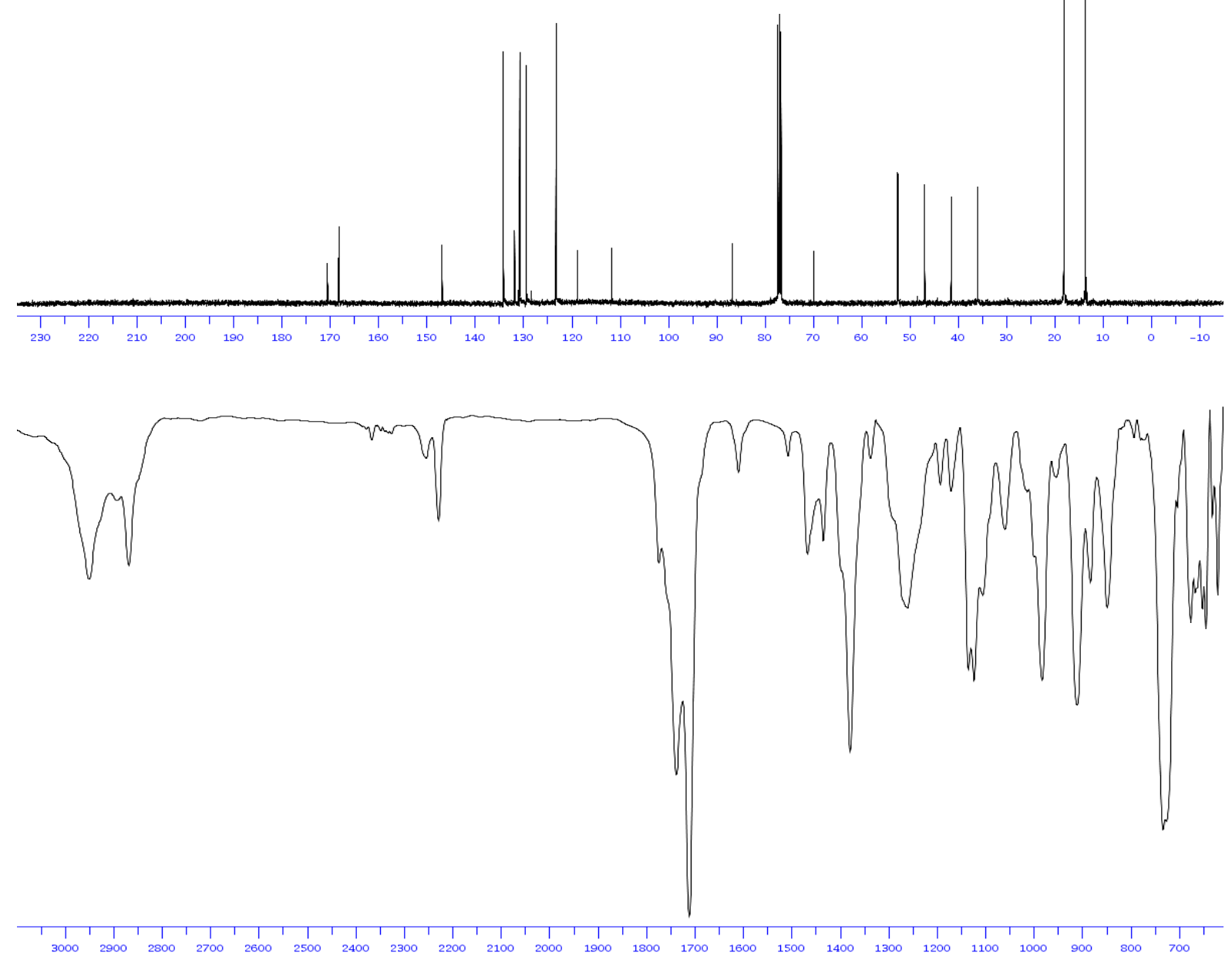
solvent: $\langle\mathrm{CDCl} 3>$
Frequency: $400.13 \mathrm{MHz}$
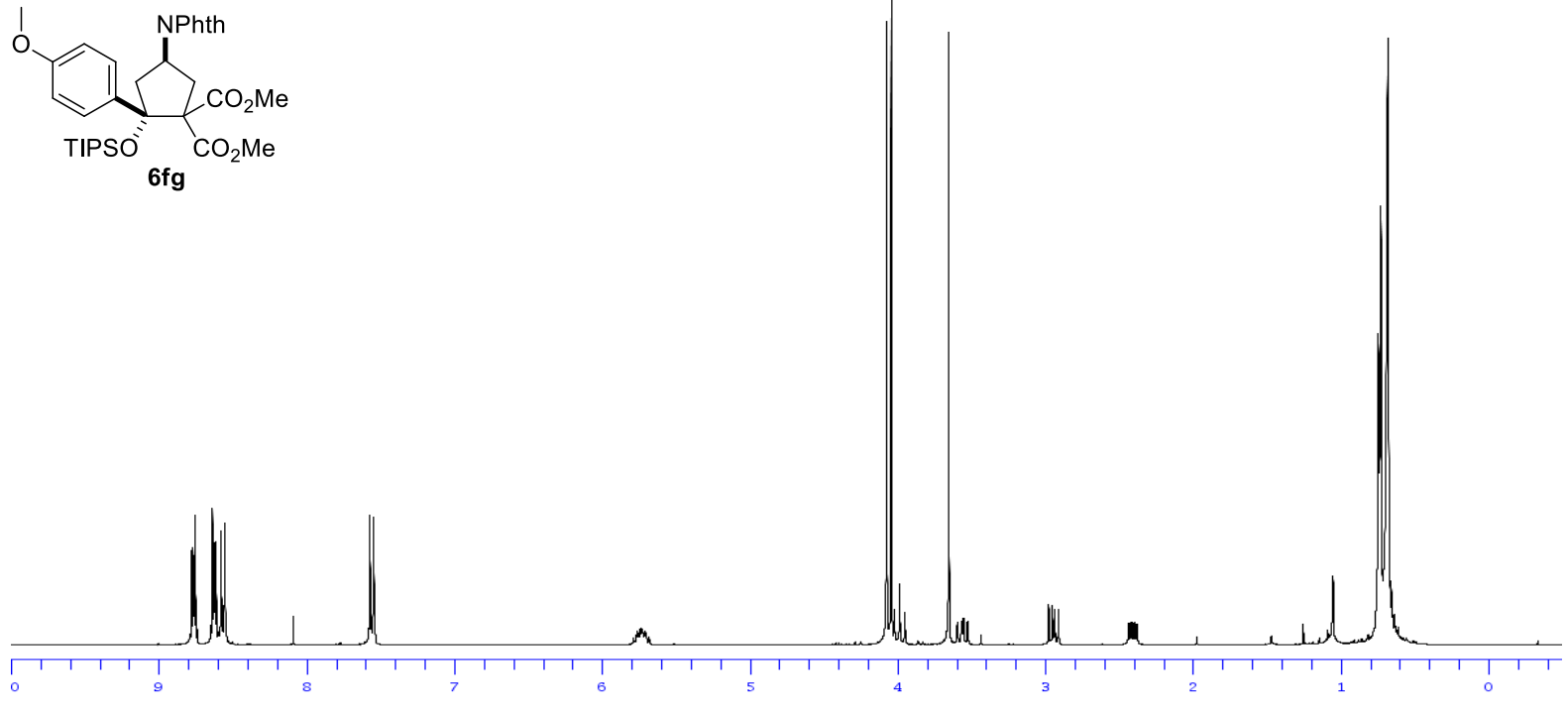

Solvent: $<$ CDCl3>
Frequency: $100.612769 \mathrm{MHz}$

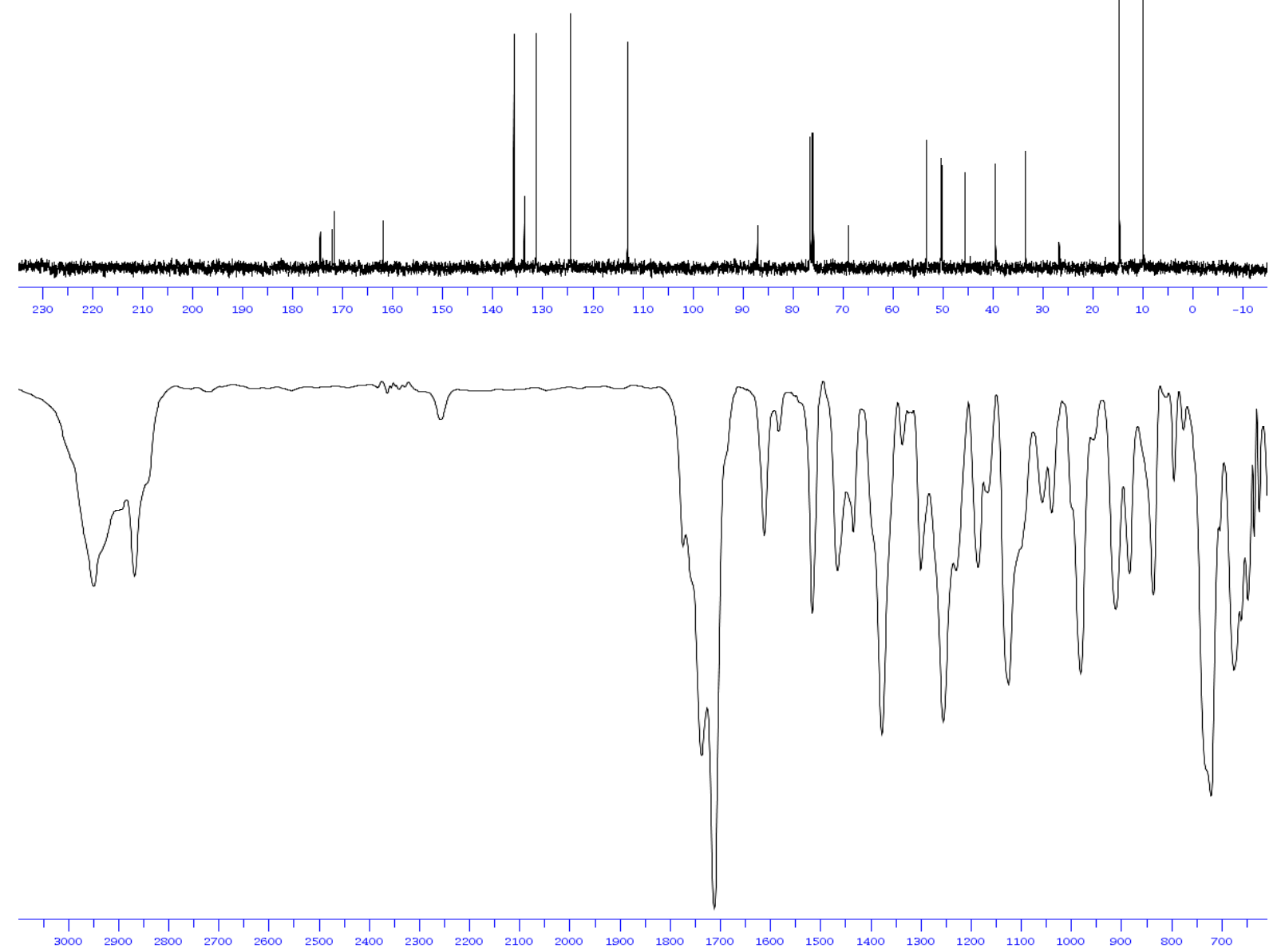


solvent: $\langle\mathrm{CDCl} 3\rangle$
Frequency: $400.13 \mathrm{MHz}$
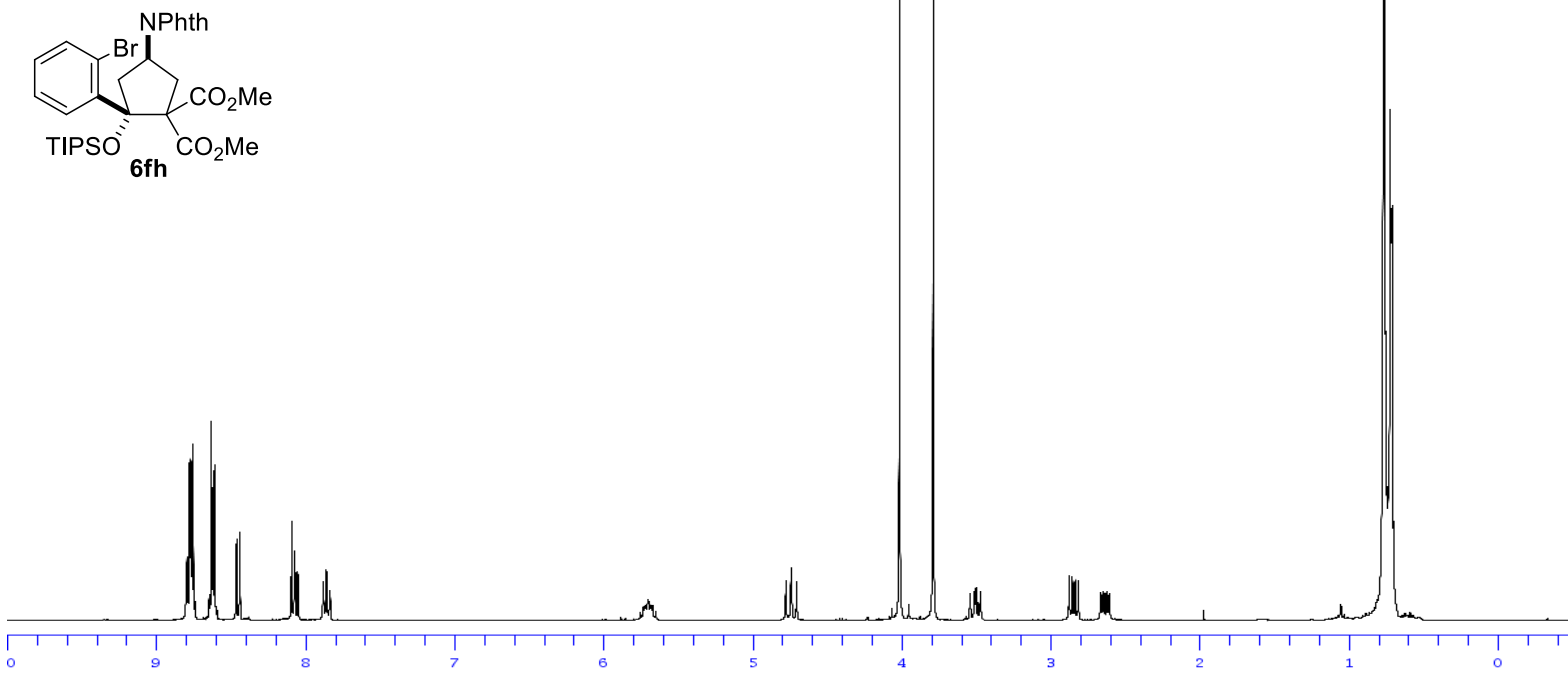

Solvent: $<C D C 13>$
Frequency 100 61 $12769 \mathrm{MHz}$
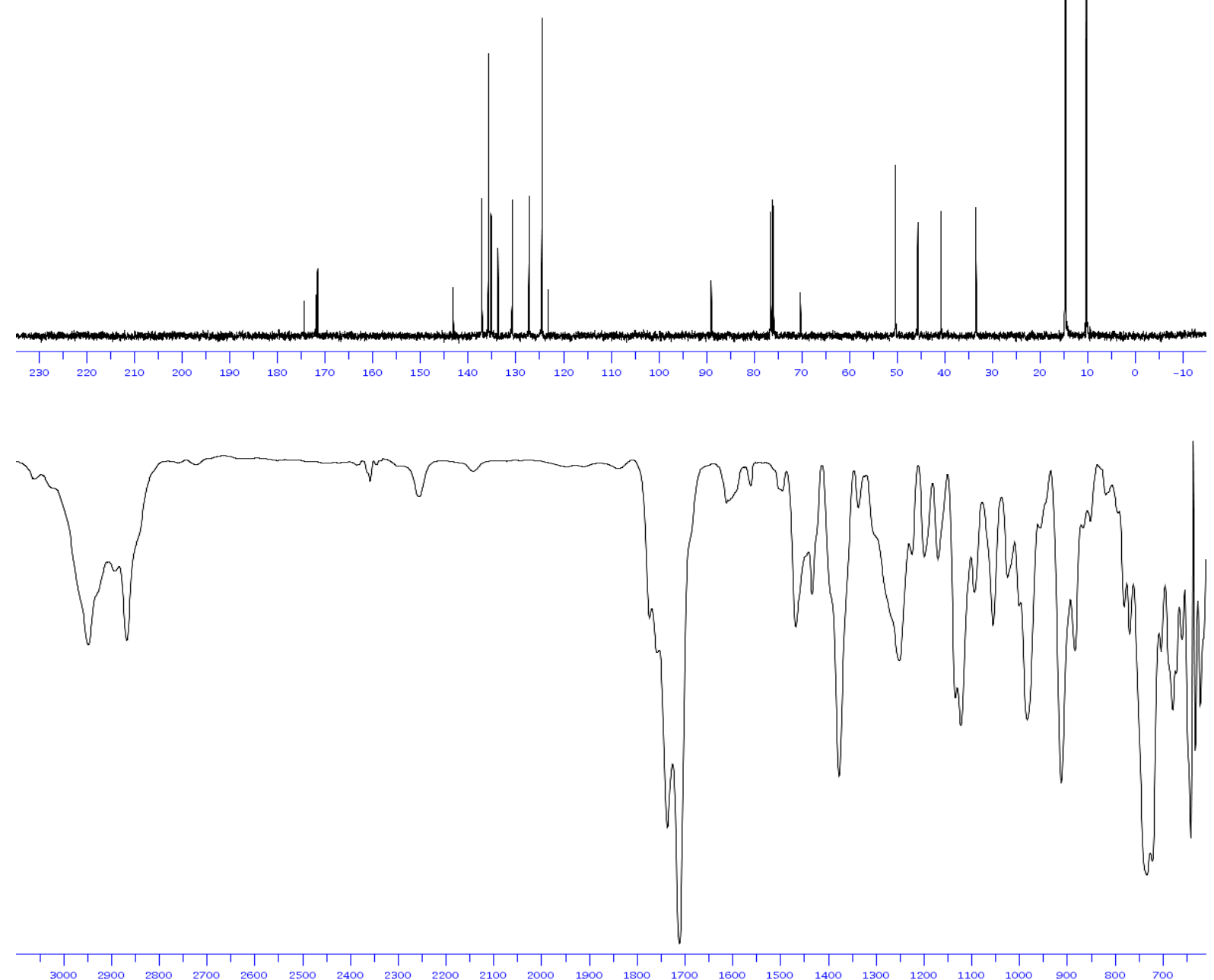
solvent: $<\mathrm{CDCl} 3>$
Frequency $400.13 \mathrm{MHz}$

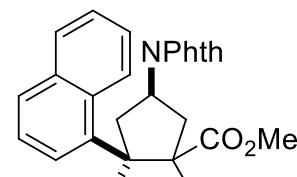

TIPSO ${ }_{6 \mathrm{fi}} \mathrm{CO}_{2} \mathrm{Me}$

Solvent: $<\mathrm{CDCl} 3>$
Frequency. $100.612769 \mathrm{MHz}$

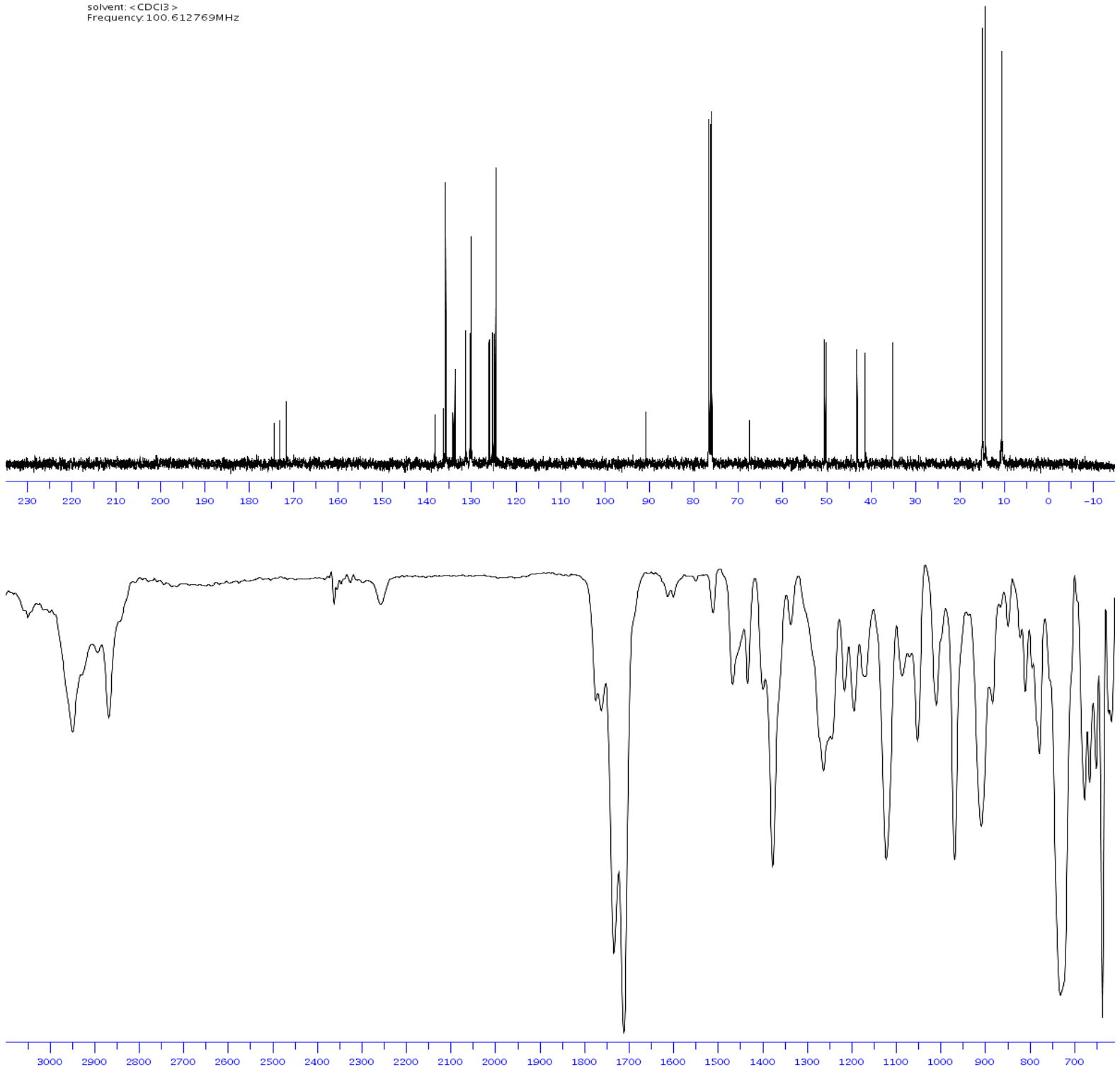


Solvent: $\langle\mathrm{CDCl}>>$
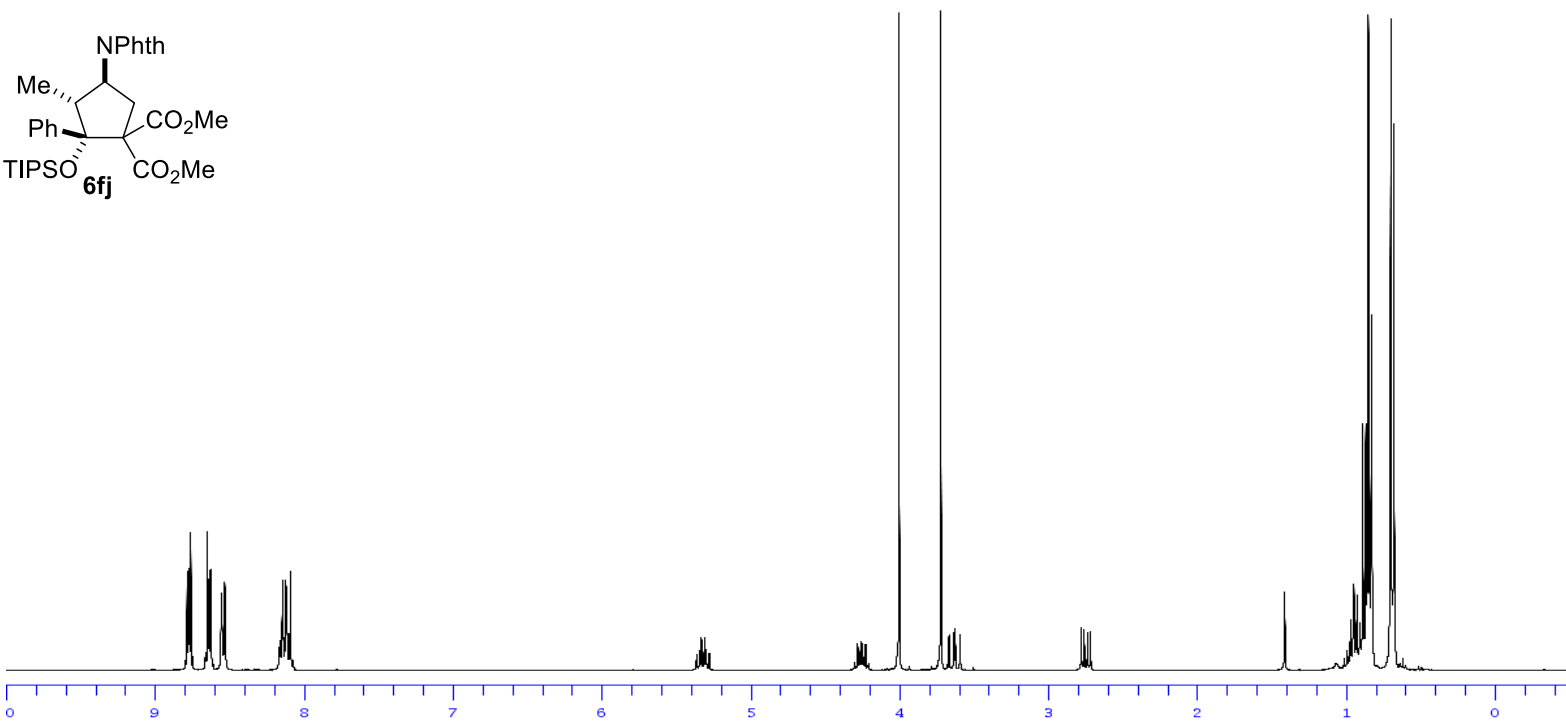

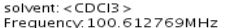
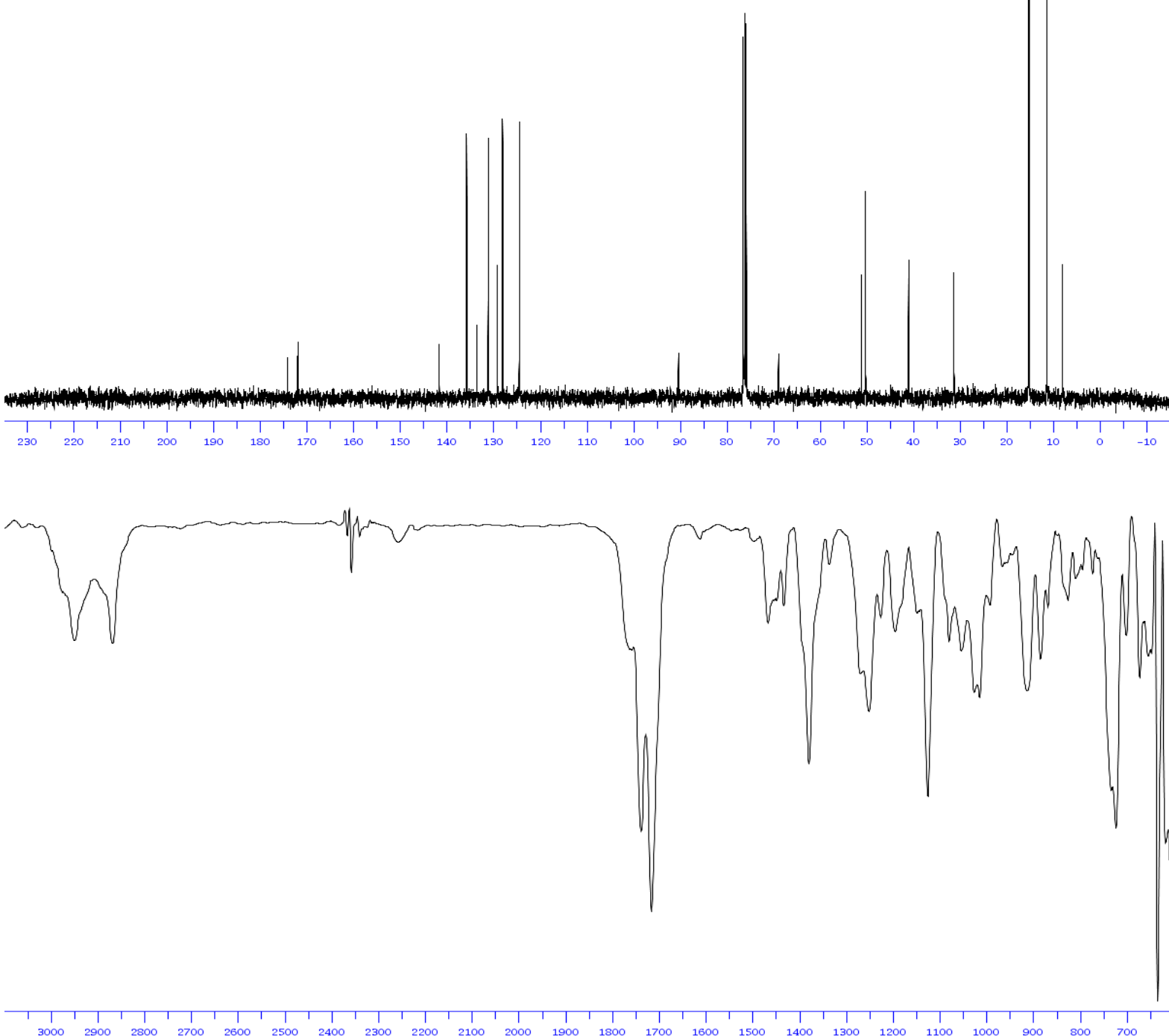
Solvent: $\langle C D C I 3>$
Frequency: $400.13 \mathrm{MHz}$
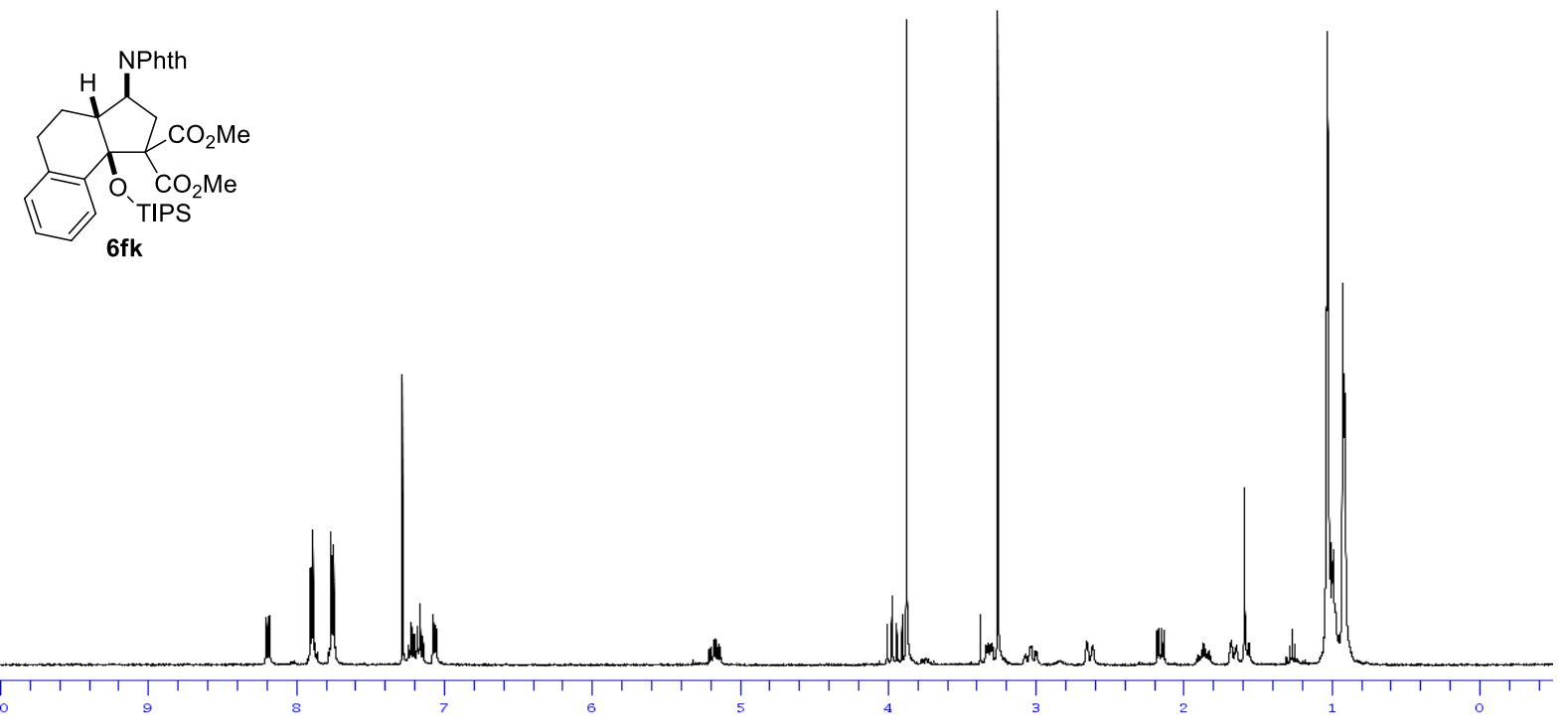

Solvent: $<C D C 13>$
Frequency. $100.612769 \mathrm{MHz}$

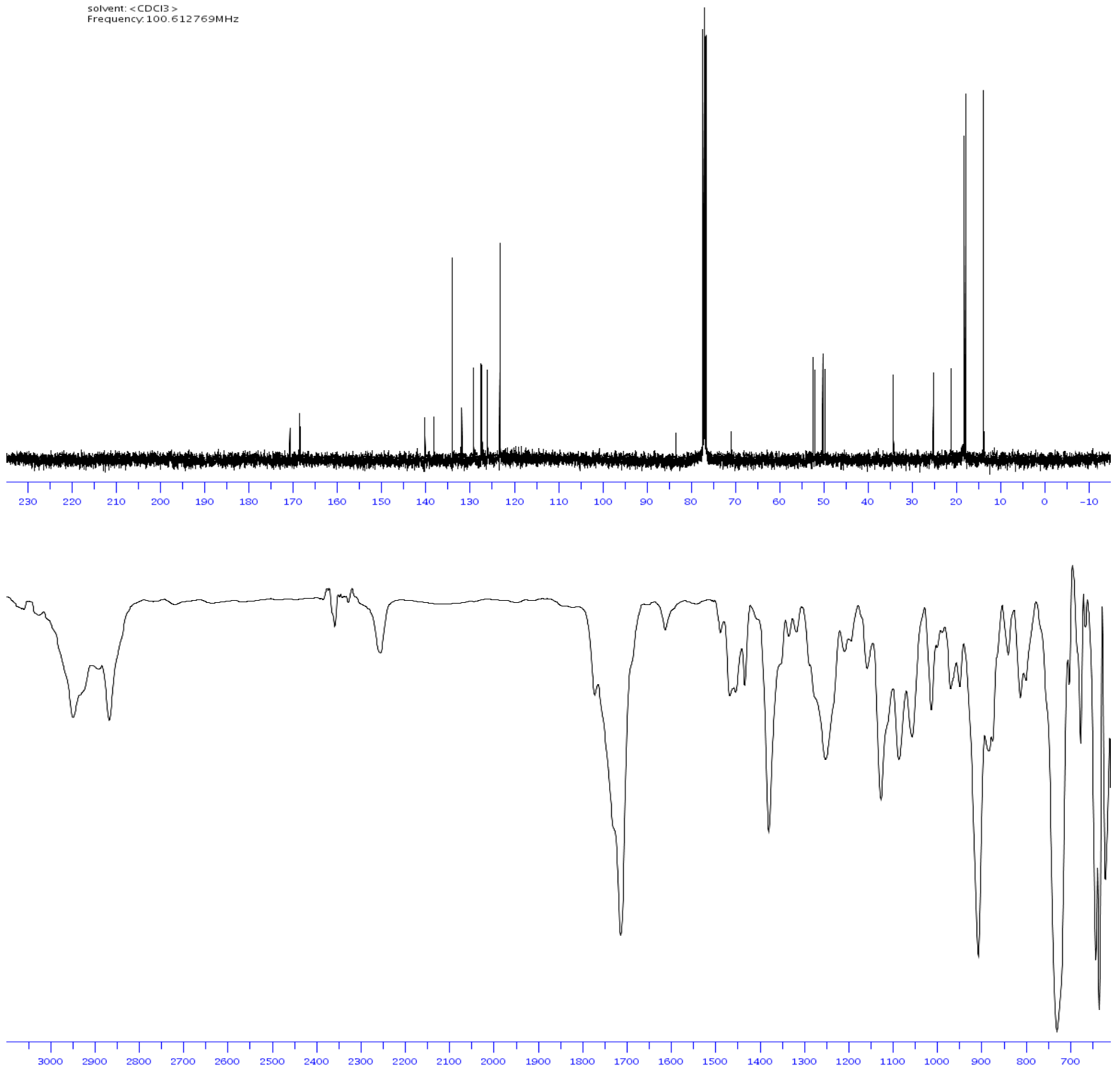


Solvent: $\angle \mathrm{CDCl} 3>$
Frequency. $400.13 \mathrm{MHz}$
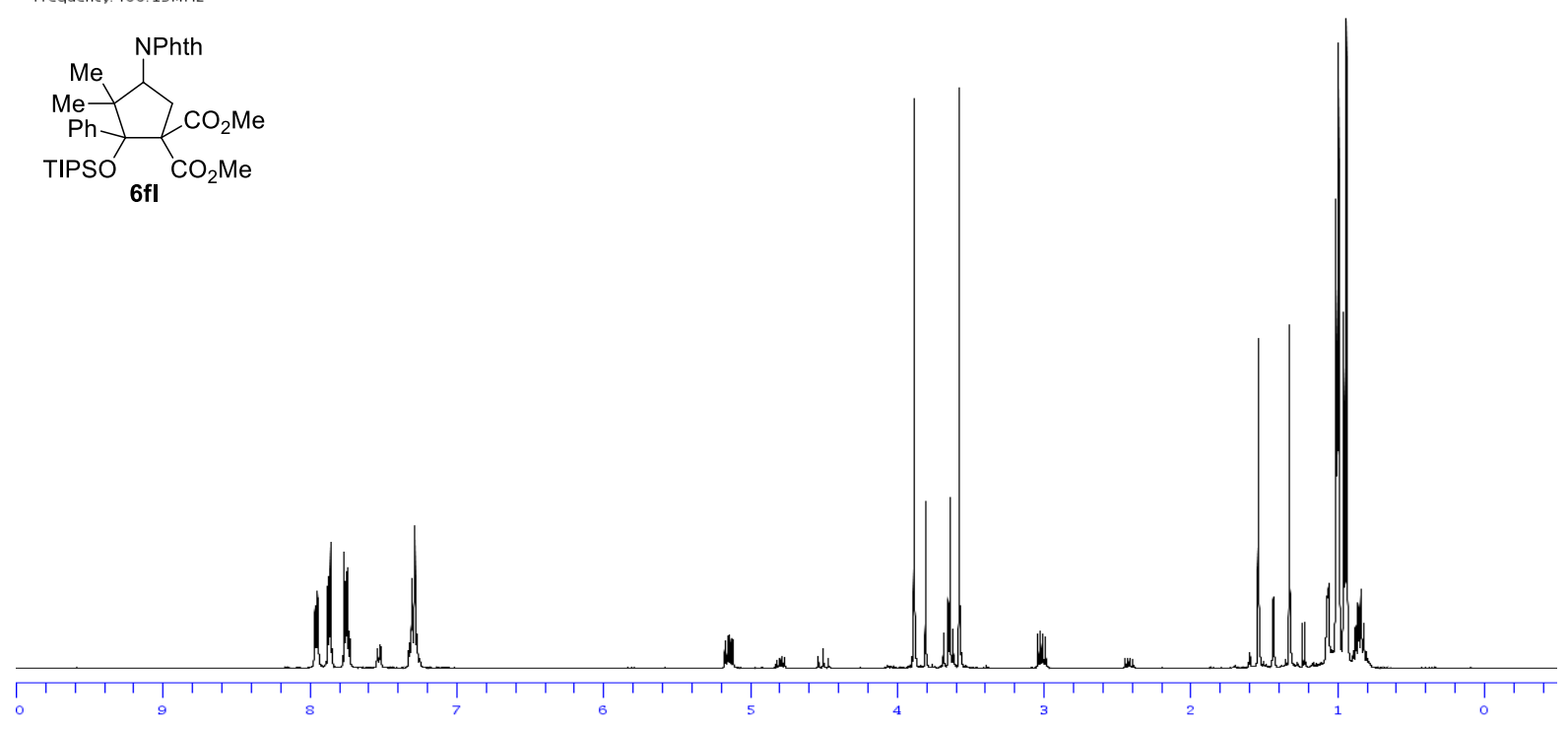

Solvent: $<\mathrm{CDCl}>$
Frequency: $100.612769 \mathrm{MHz}$

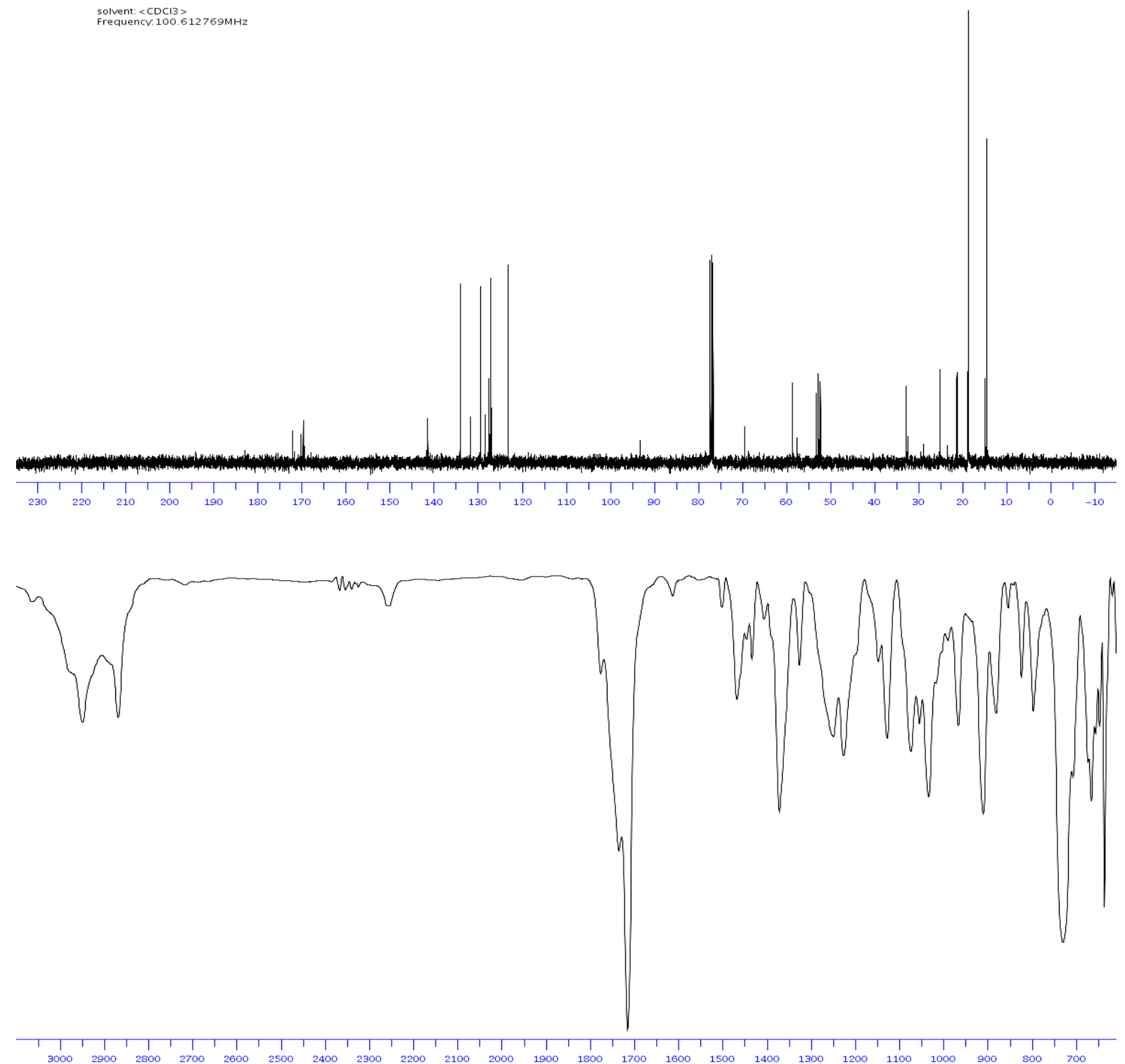


solvent: $<C D C I 3\rangle$
Frequency: $400.13 \mathrm{MHz}$
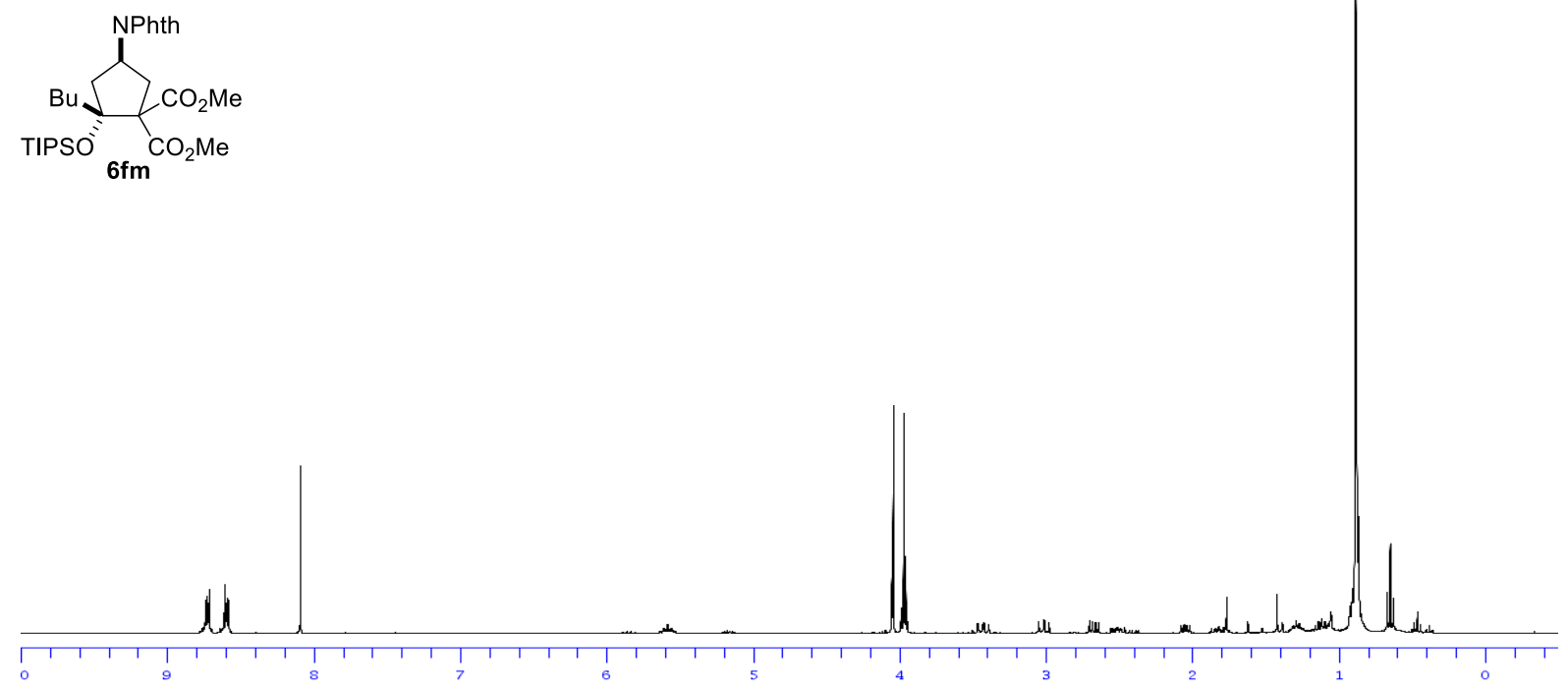

Solwent: $<\mathrm{CDCl} 3>$
Frequency. $100.612769 \mathrm{MHz}$
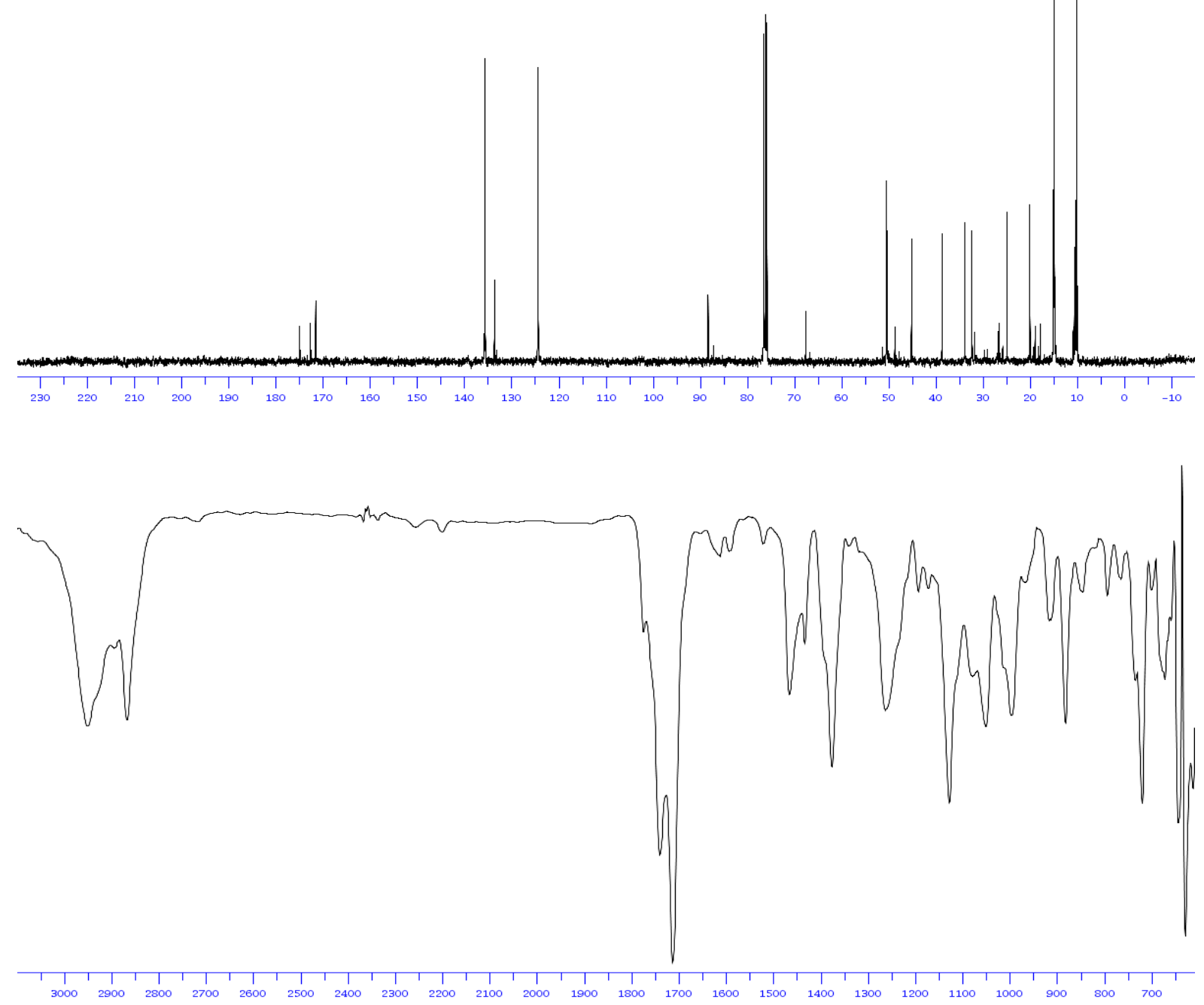


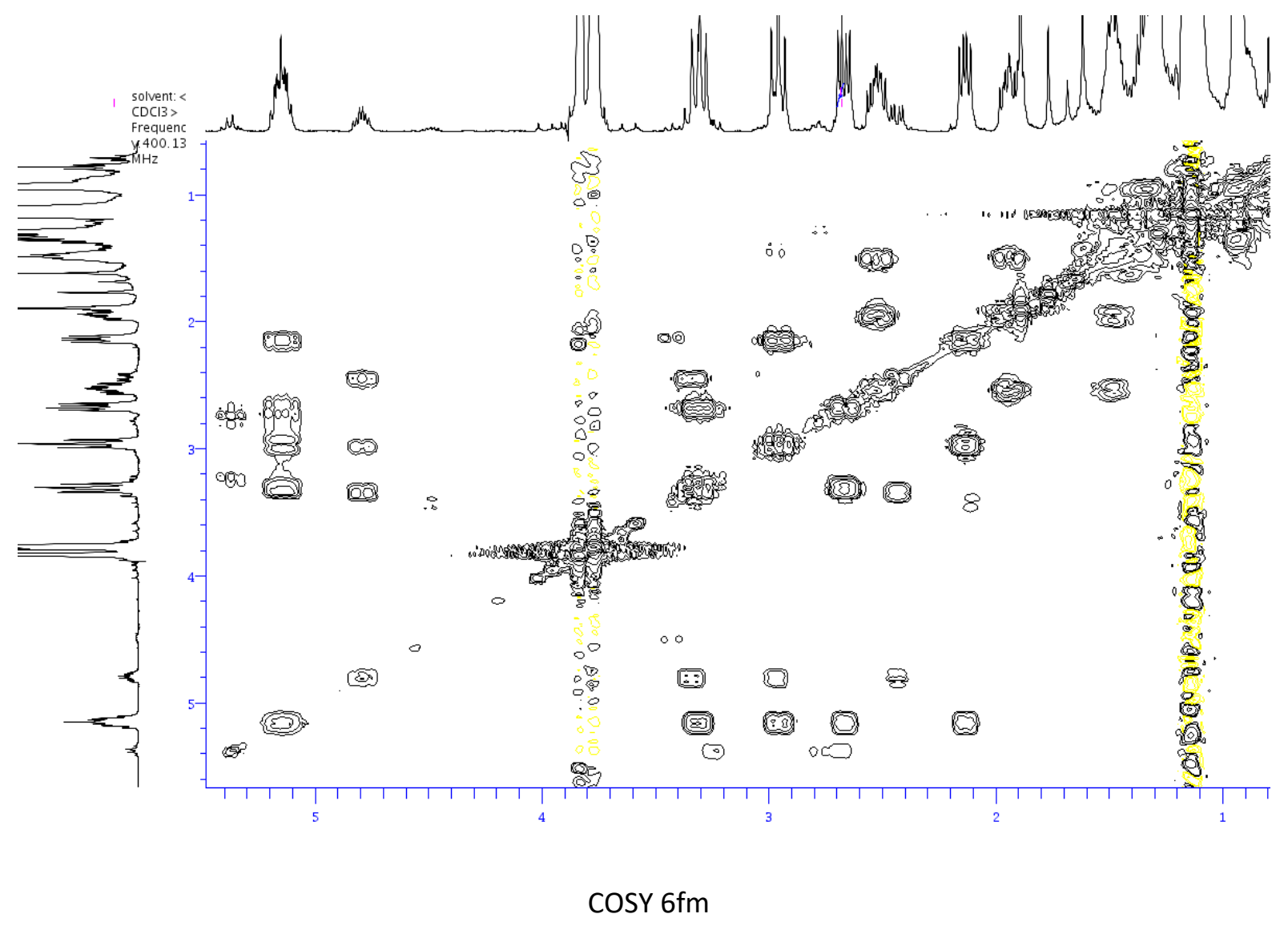


Solvent: $\langle\mathrm{CDCl} 3\rangle$
Frequency: $400.13 \mathrm{MH}$

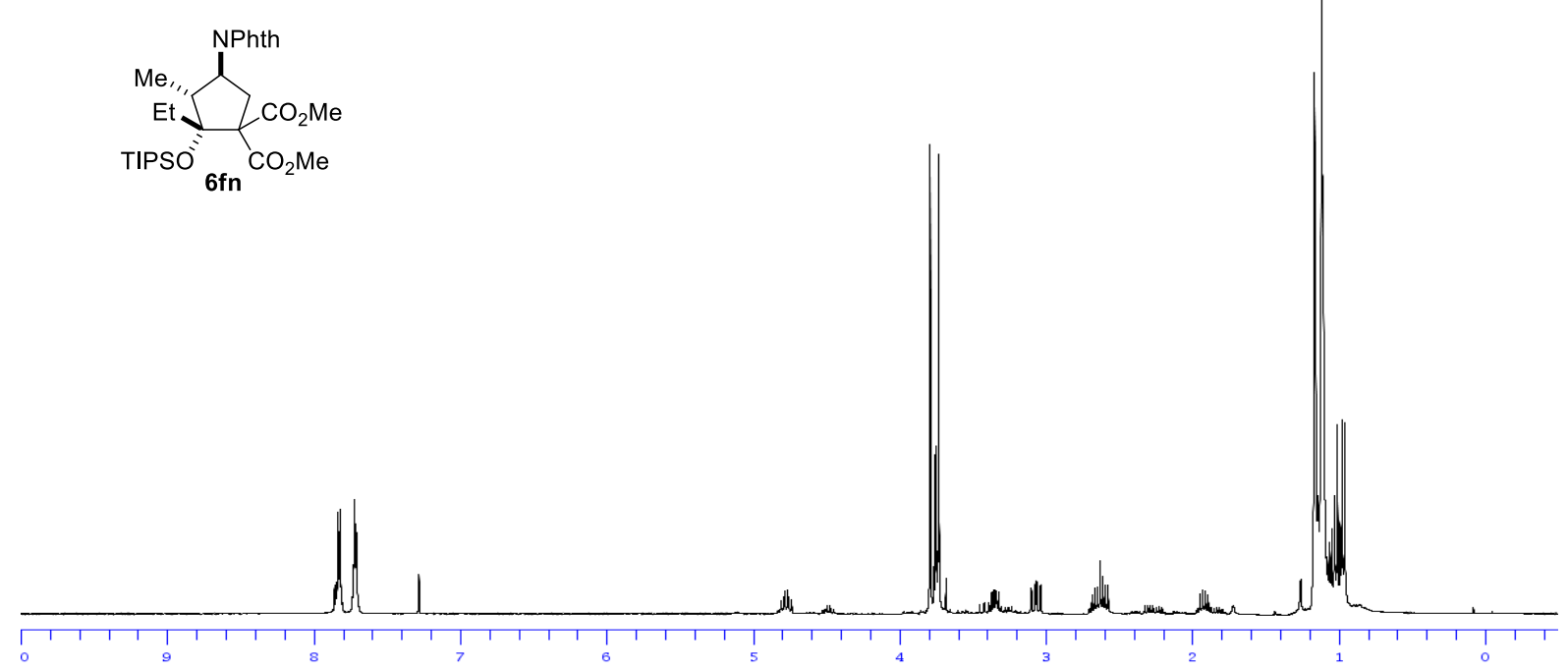

solvent: $<\mathrm{CDCl} 3>$
Frequency: $100.612769 \mathrm{MHz}$
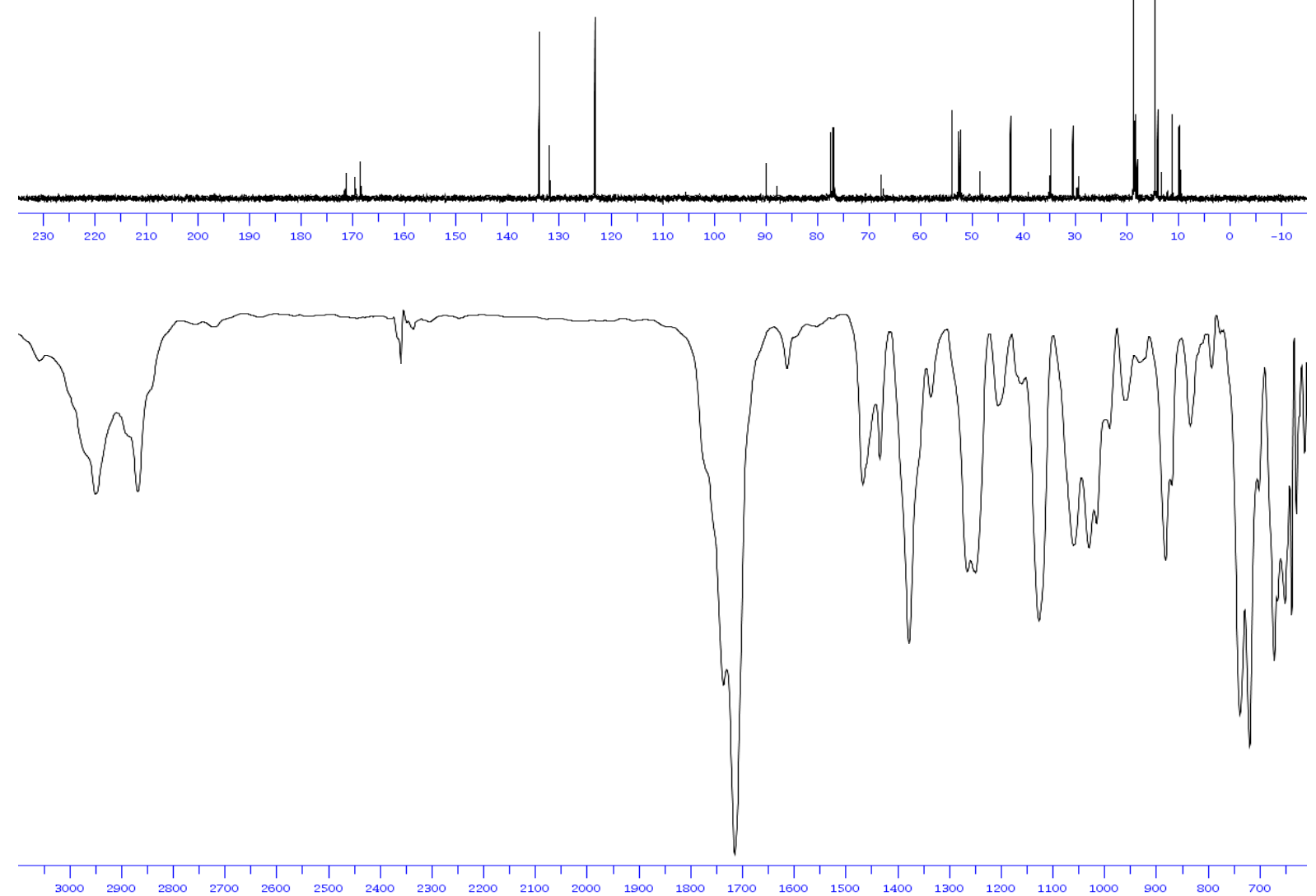

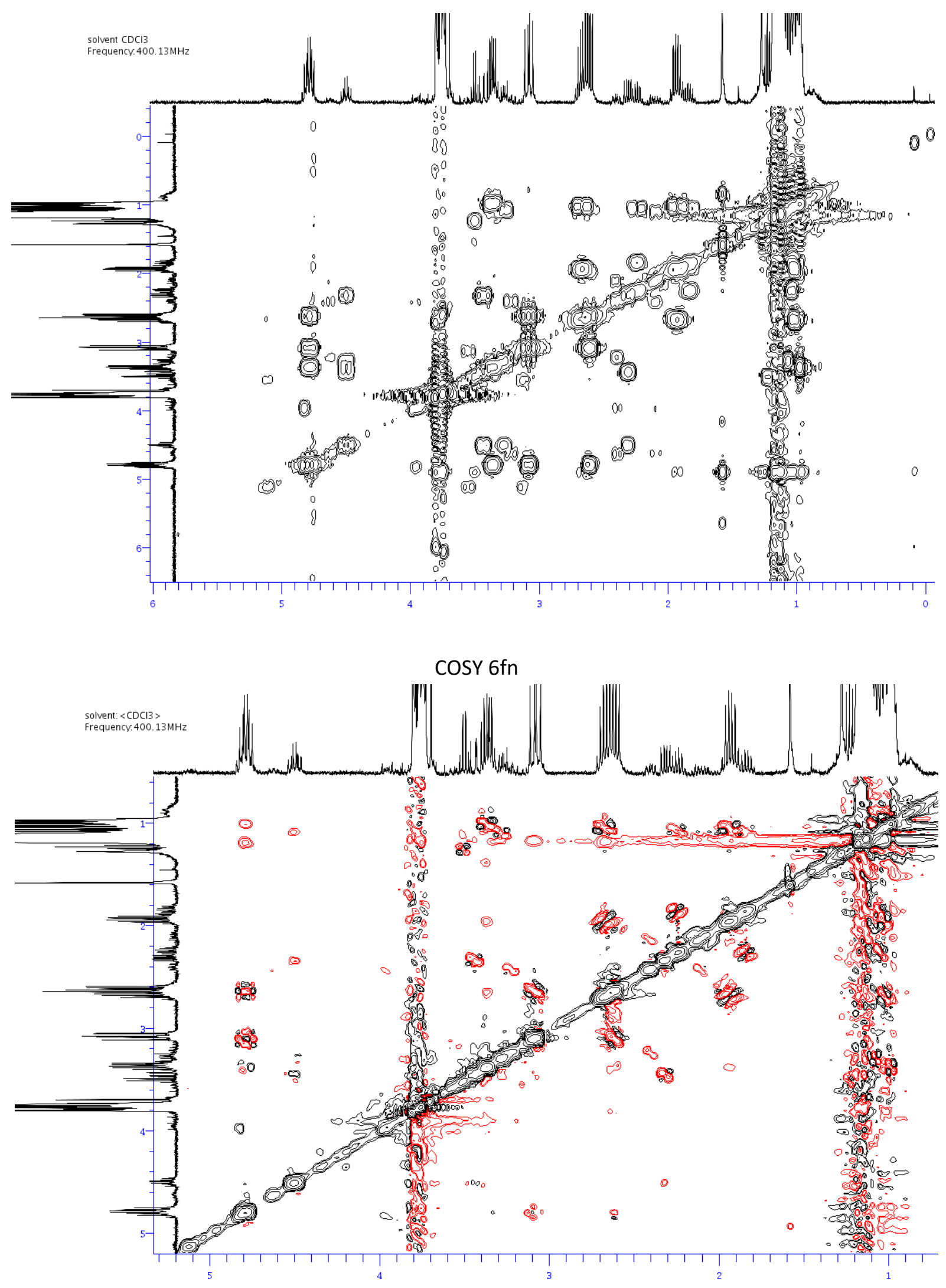

ROESY $6 \mathrm{fn}$ 
solvent: $\langle\mathrm{CDCl} 3\rangle$
Frequency: $400.13 \mathrm{MHz}$
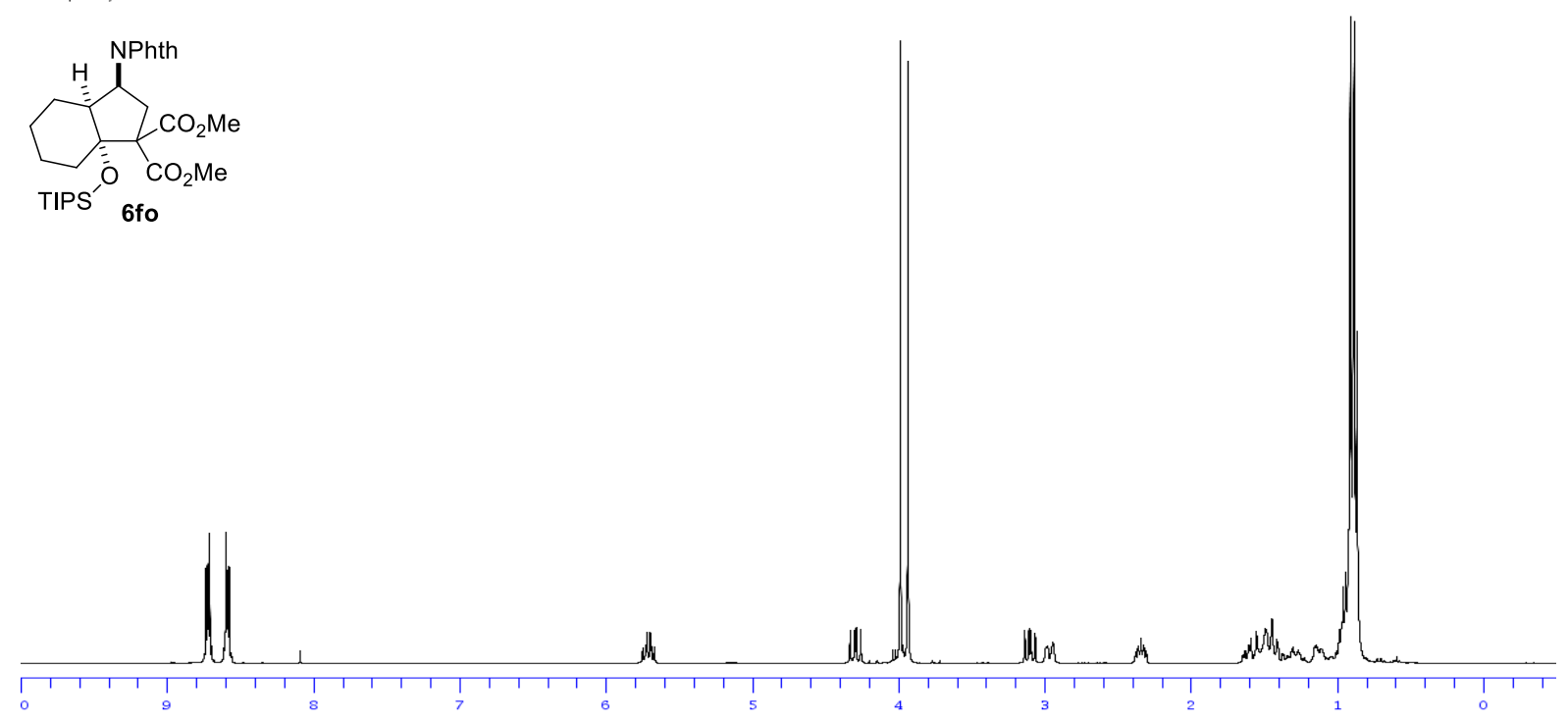

solvent: $<$ CDCI3>
Frequency: $100.612769 \mathrm{MHz}$
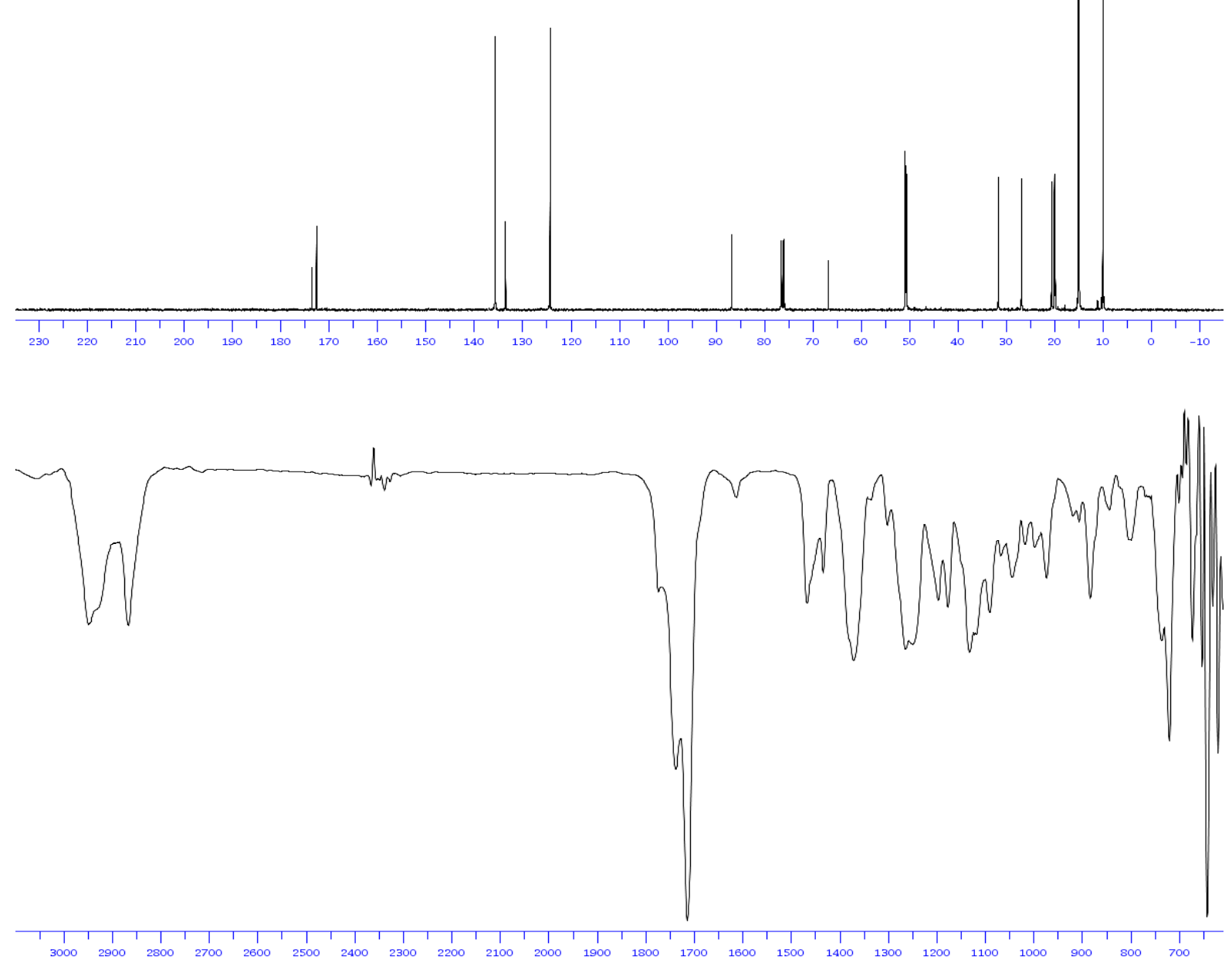
solvent: $\langle\mathrm{CDCl} 3\rangle$
Frequency: $400.13 \mathrm{MHz}$
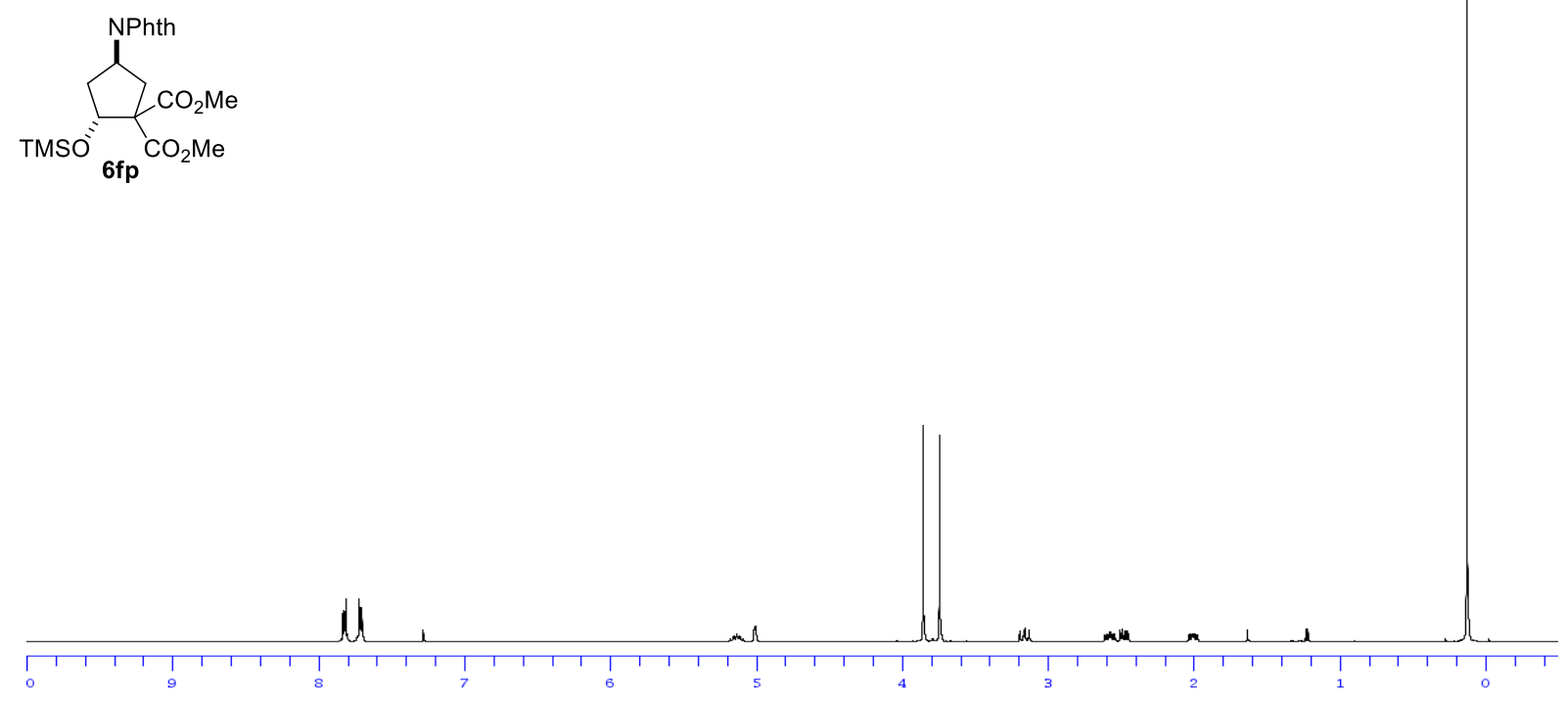

solvent: $<$ CDCI3 $>$
FrequencY: $100.612769 \mathrm{MHz}$
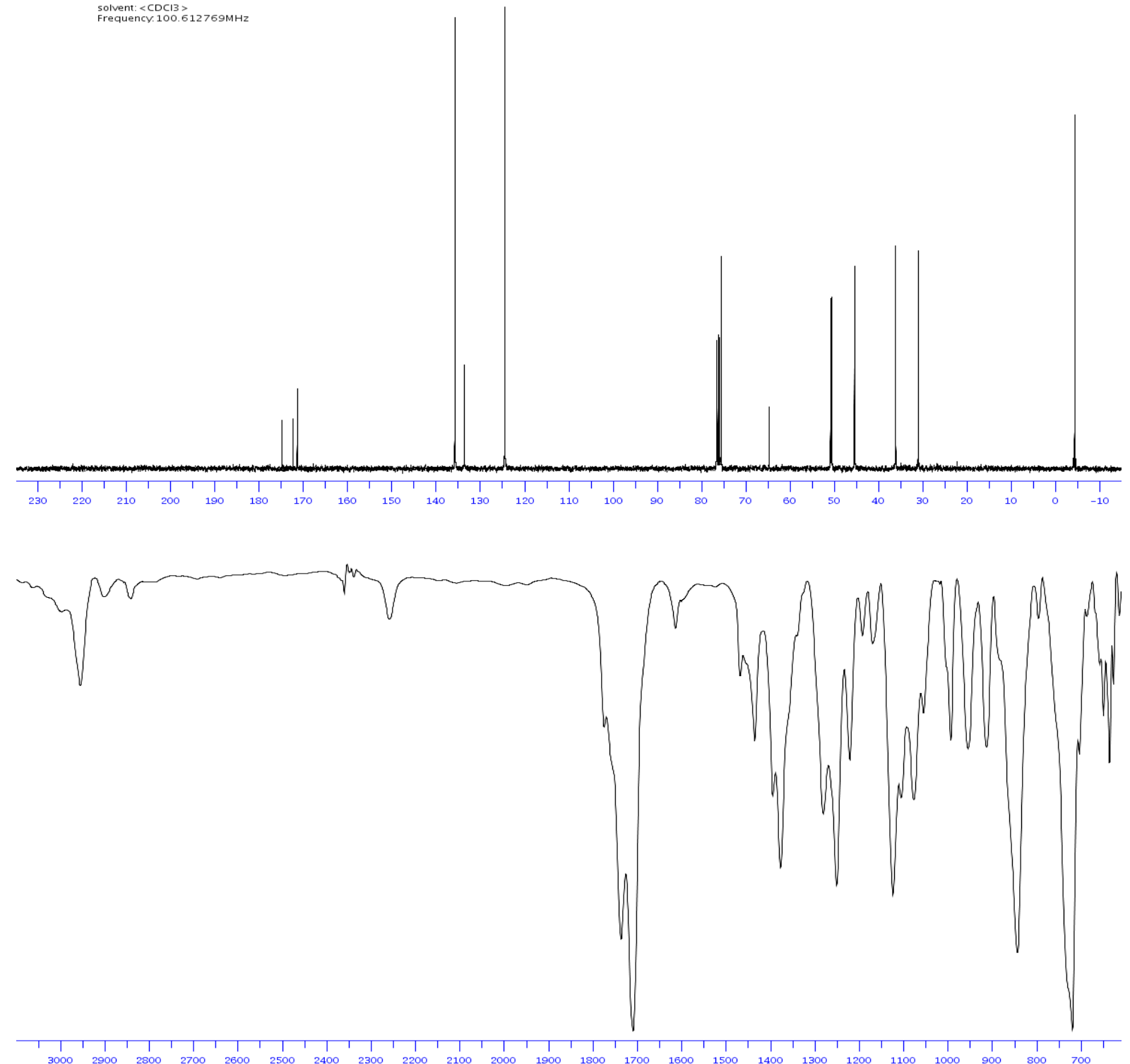


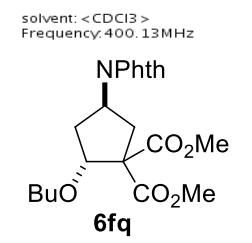

aculdel an de

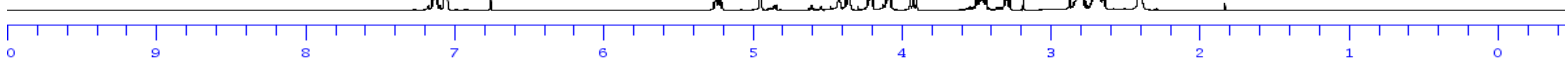

solvent: $<$ CDCI3 $>$
Frequency: $100.612769 \mathrm{MHz}$

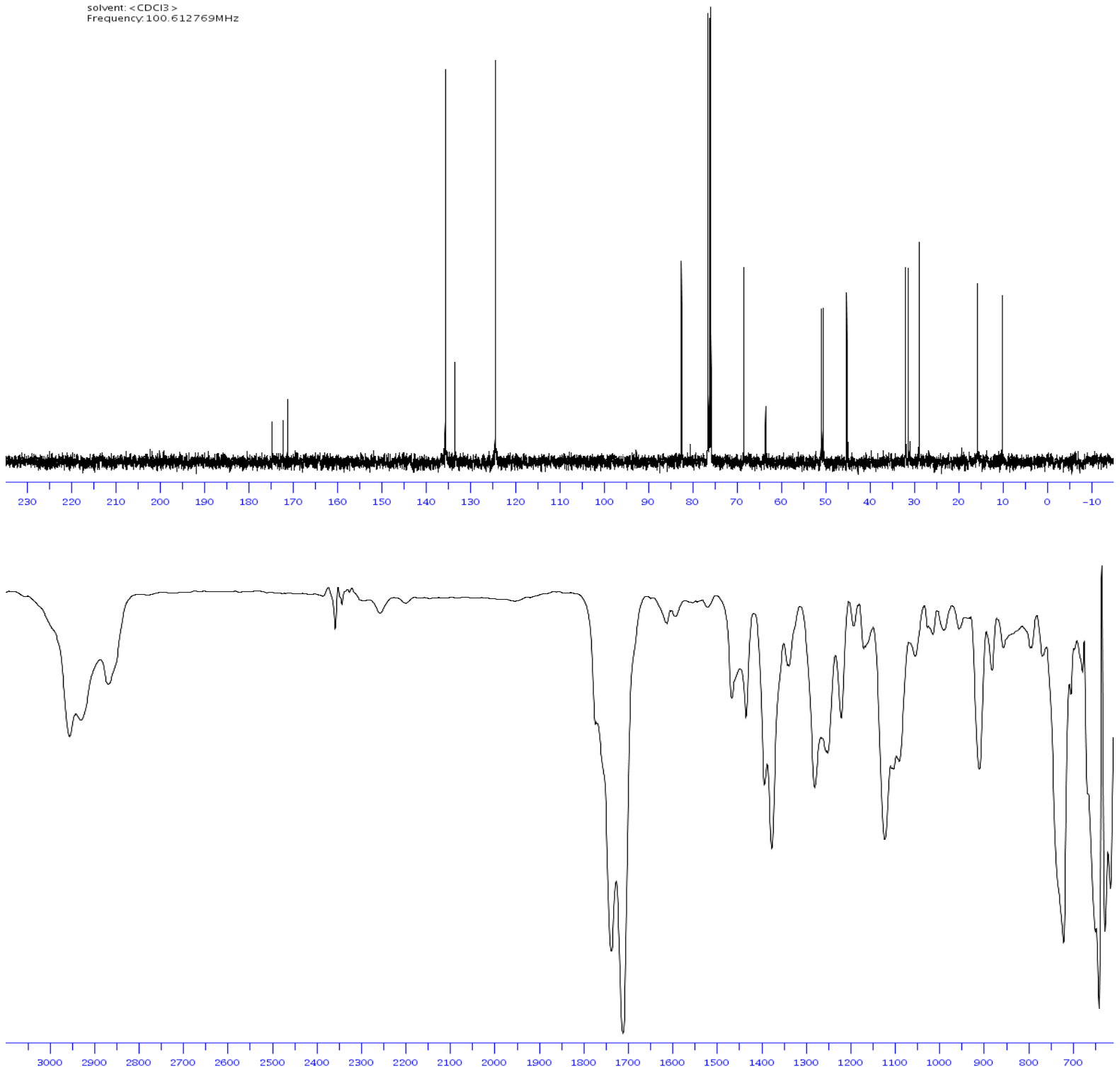


Solvent: $\angle C D C l 3>$
Frequency. $400.13 \mathrm{MHz}$

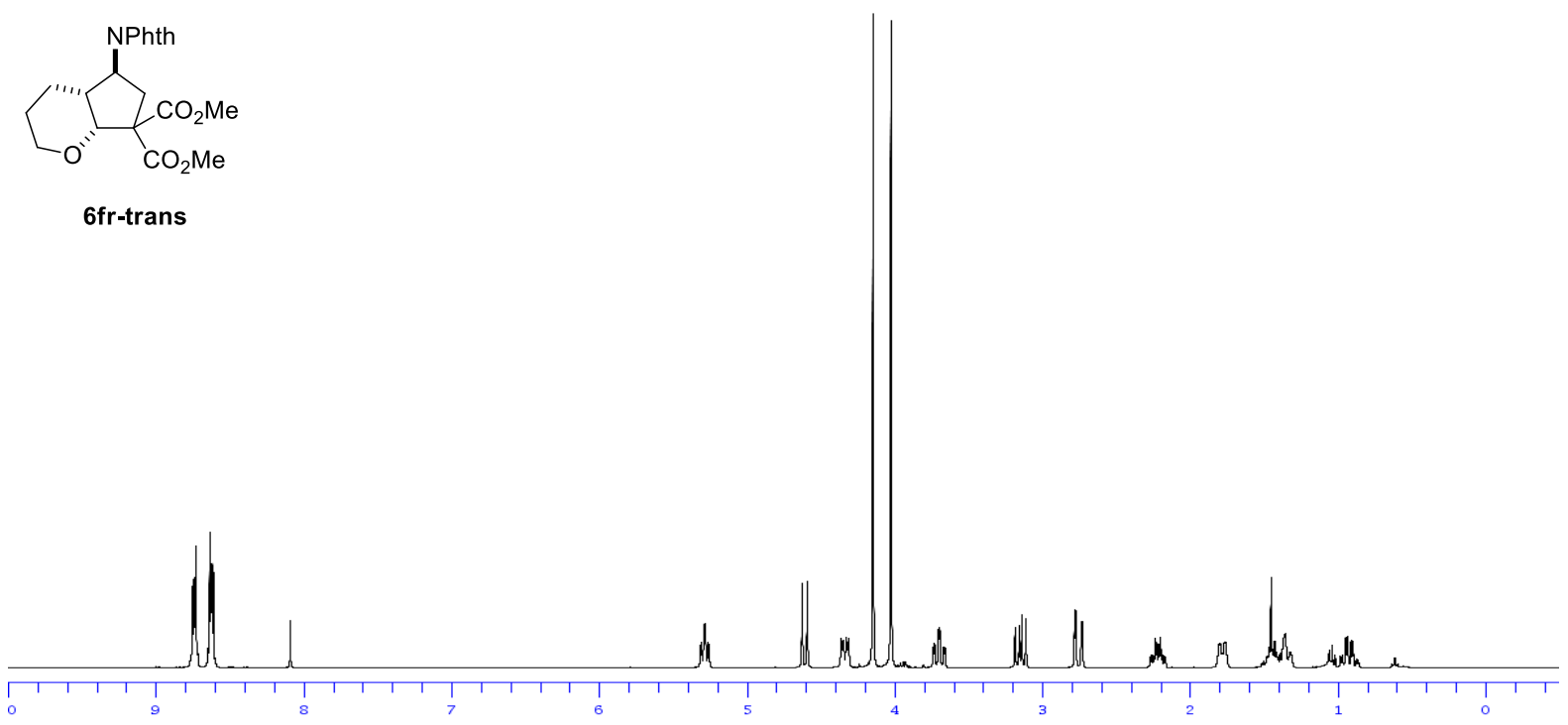

solwent: $<$ CDCI3>
FrequencY. $100.612769 \mathrm{MHz}$

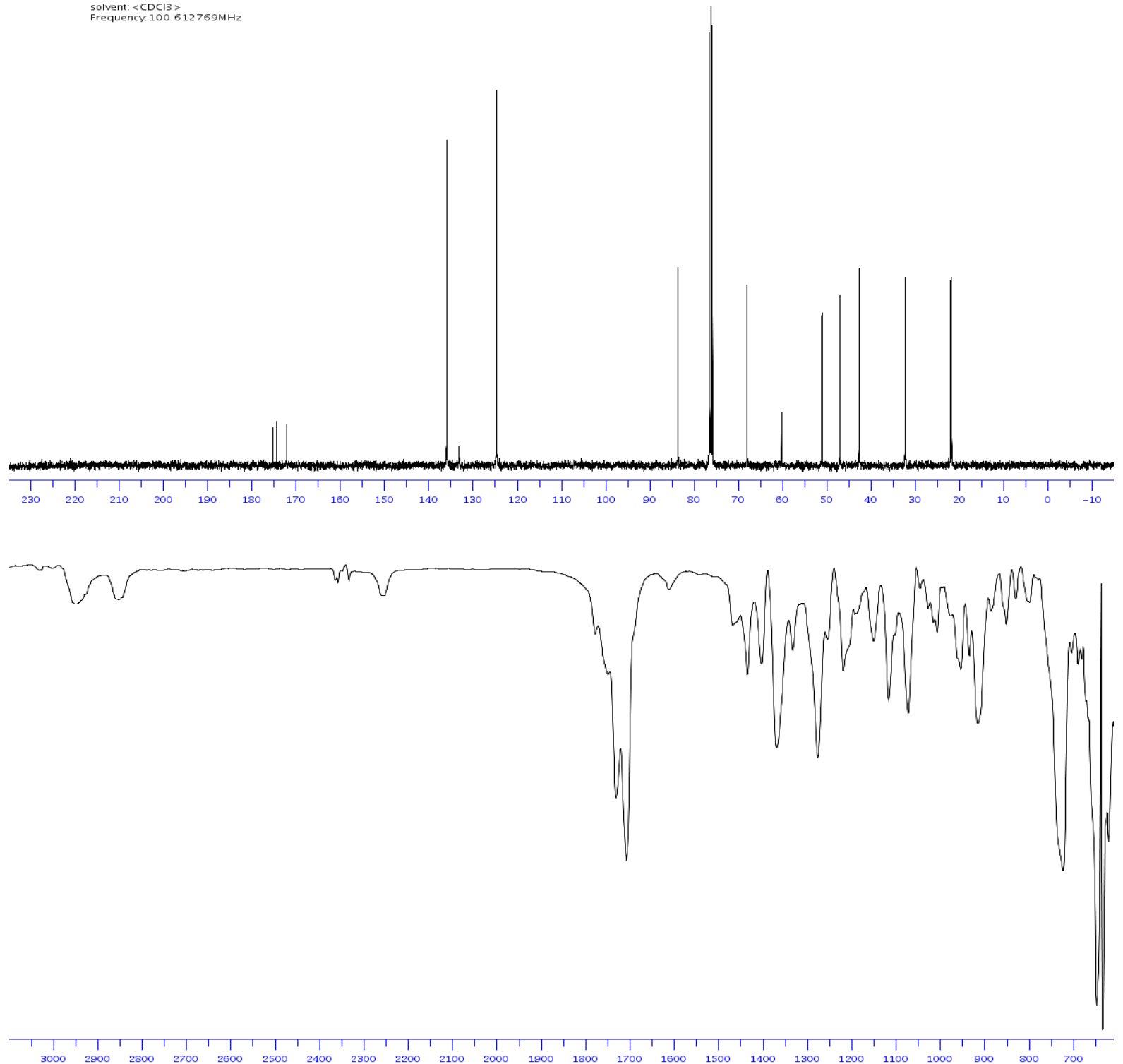


solvent: $\langle\mathrm{CDCl} 3\rangle$
Frequency: $400.13 \mathrm{MHz}$

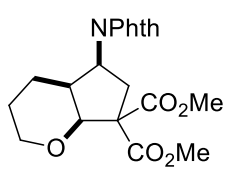

$6 \mathrm{fr}$-cis

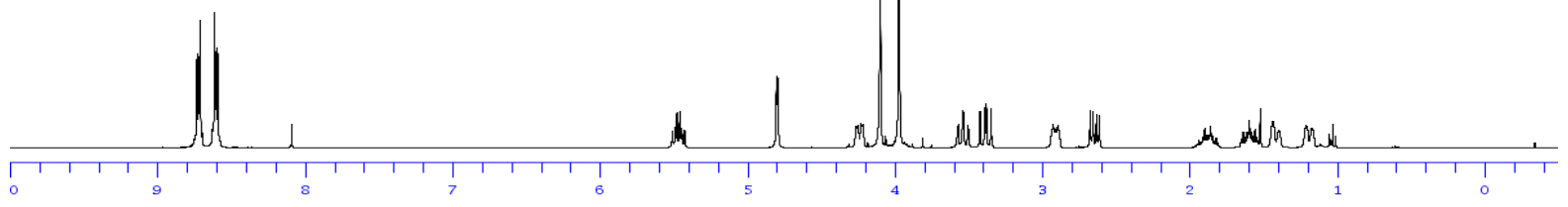

Solvent: $<\mathrm{CDCl} 3>$
Frequency: $100.612769 \mathrm{MHz}$
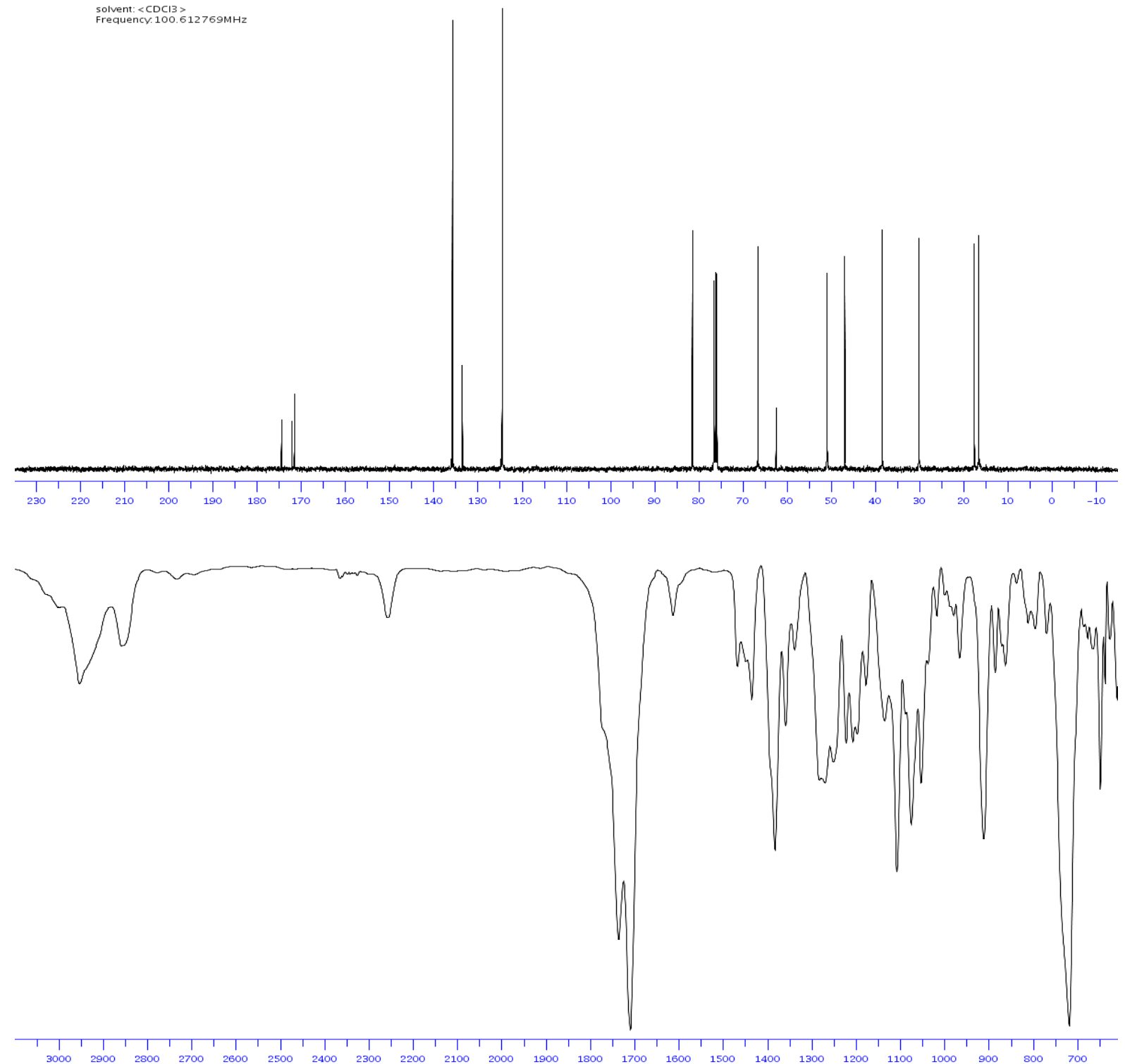
<smiles>CCOC(=O)C(CC(CC(=O)c1ccccc1)Nc1ccccc1)C(=O)OCC</smiles>

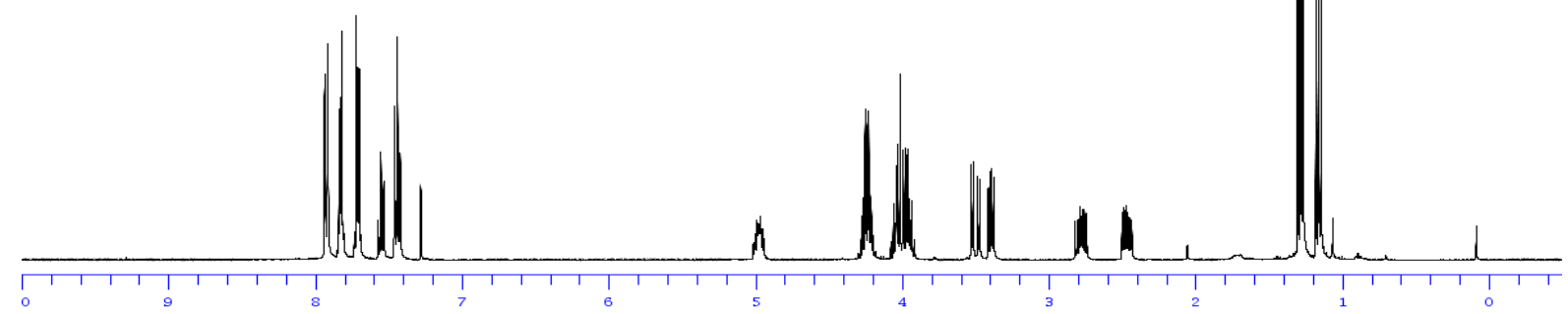

Solvent: $<\mathrm{CDCl} 3>$
Frequency: $100.612769 \mathrm{MHz}$

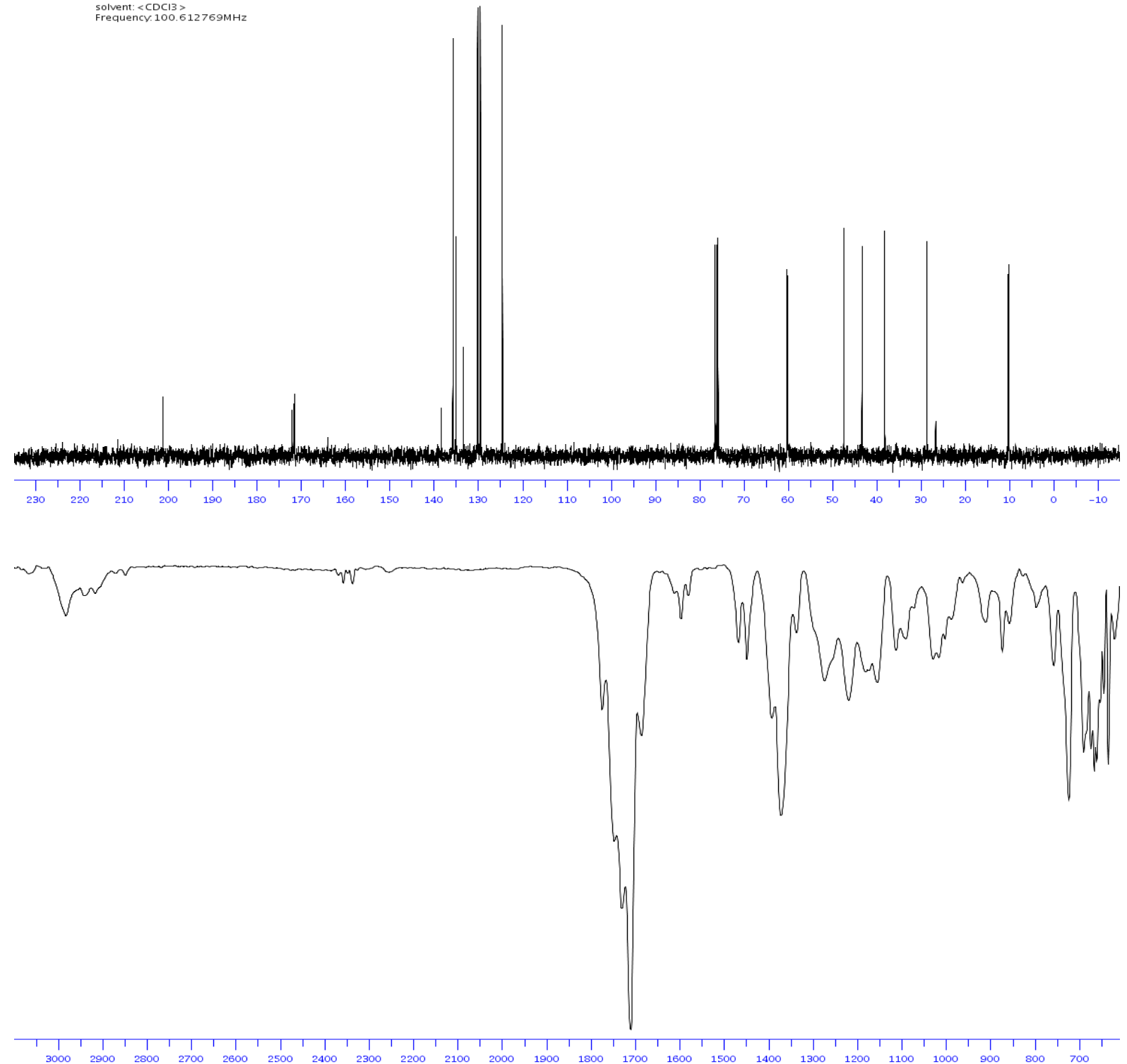


solvent: $<C D C l 3>$
Frequency. $400.03 \mathrm{MHz}$
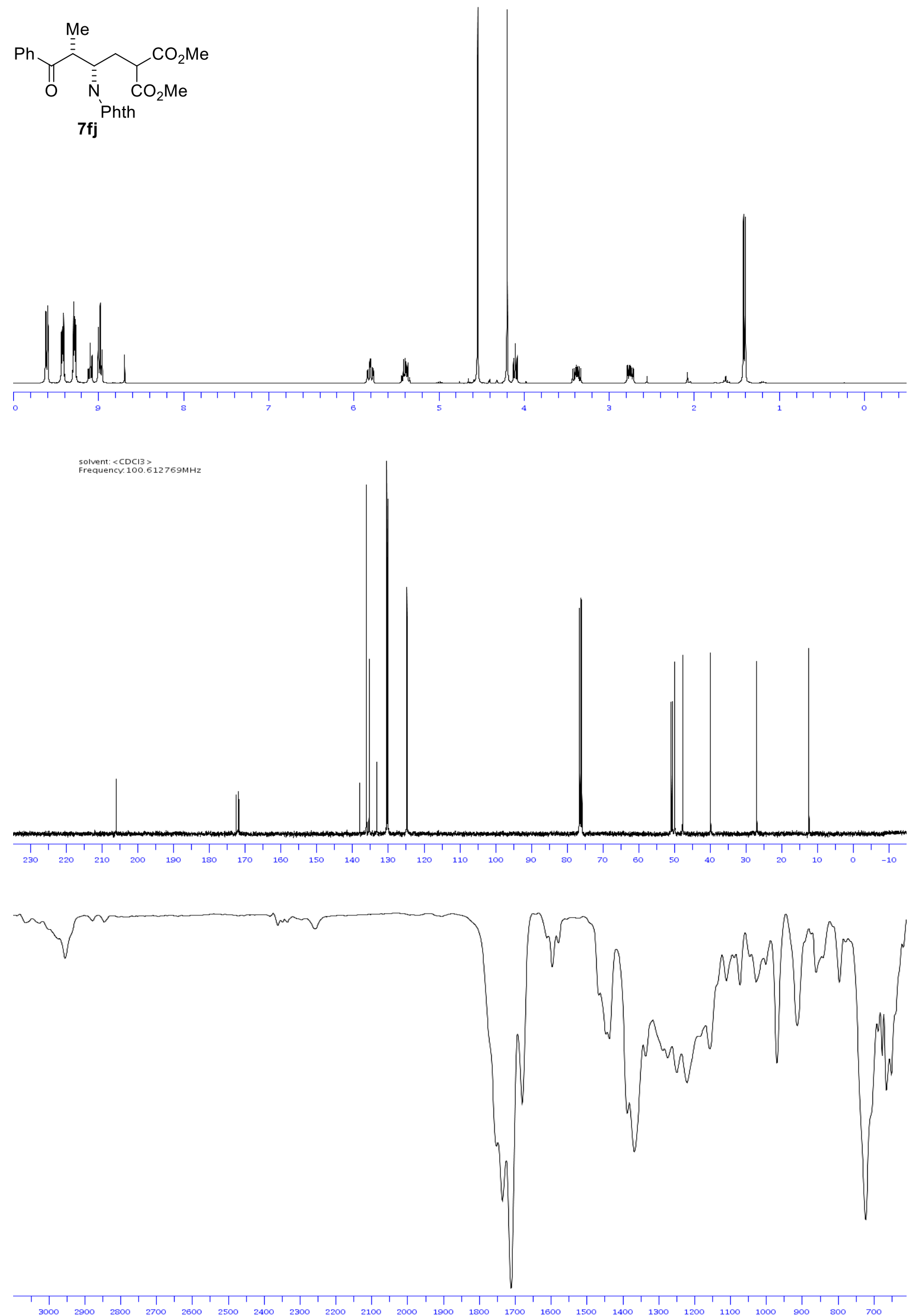
Solvent: $\langle C \mathrm{CDCl} 3\rangle$
Frequency: $400.13 \mathrm{MHz}$

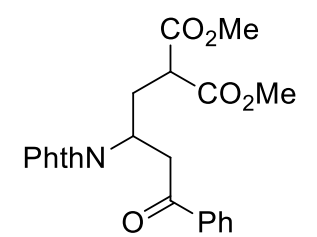

7fa: $24 \%$

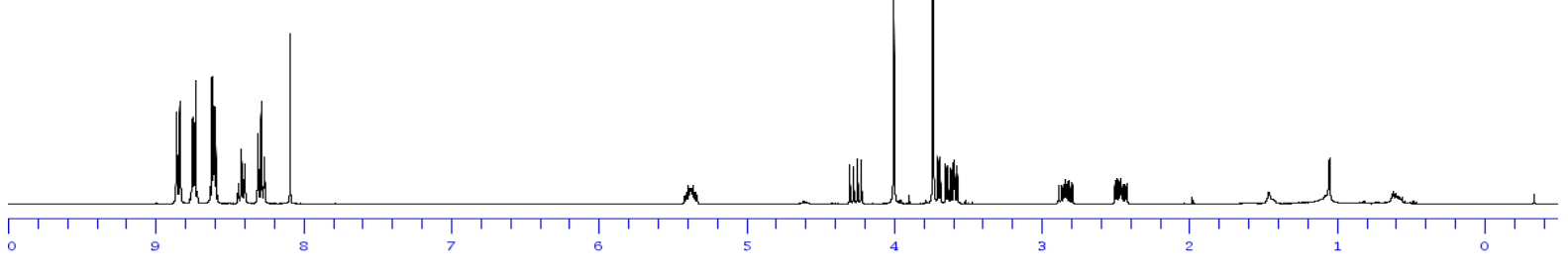

Solvent: $<$ CDC13 $>$
Frequency: $100.612769 \mathrm{MHz}$
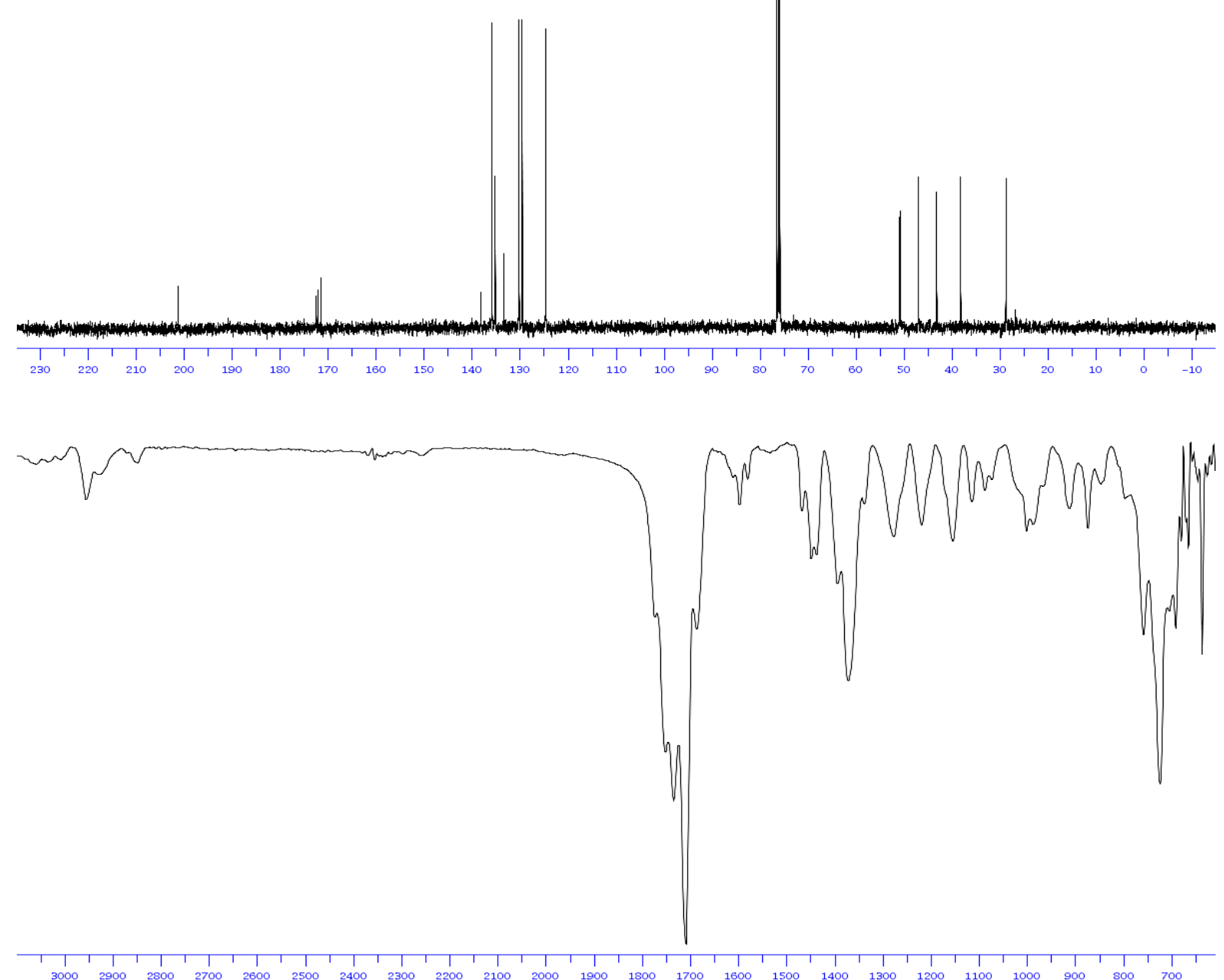
Solvent: $<C D C l 3>$
Frequency. $400.13 \mathrm{MHz}$<smiles>COC(=O)C1(C(=O)OC)CC(N)C[C@]1(OC(F)(F)F)c1ccccc1</smiles>

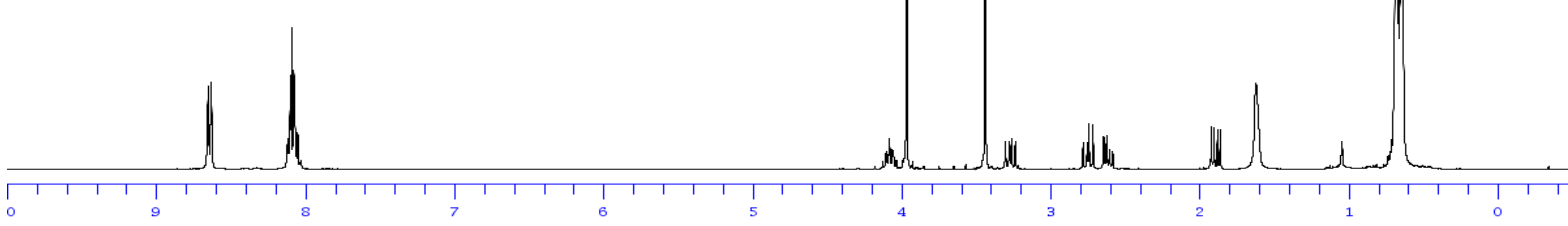

solvent: $<\mathrm{CDCl} 3>$
Frequency: $100.612769 \mathrm{MHz}$
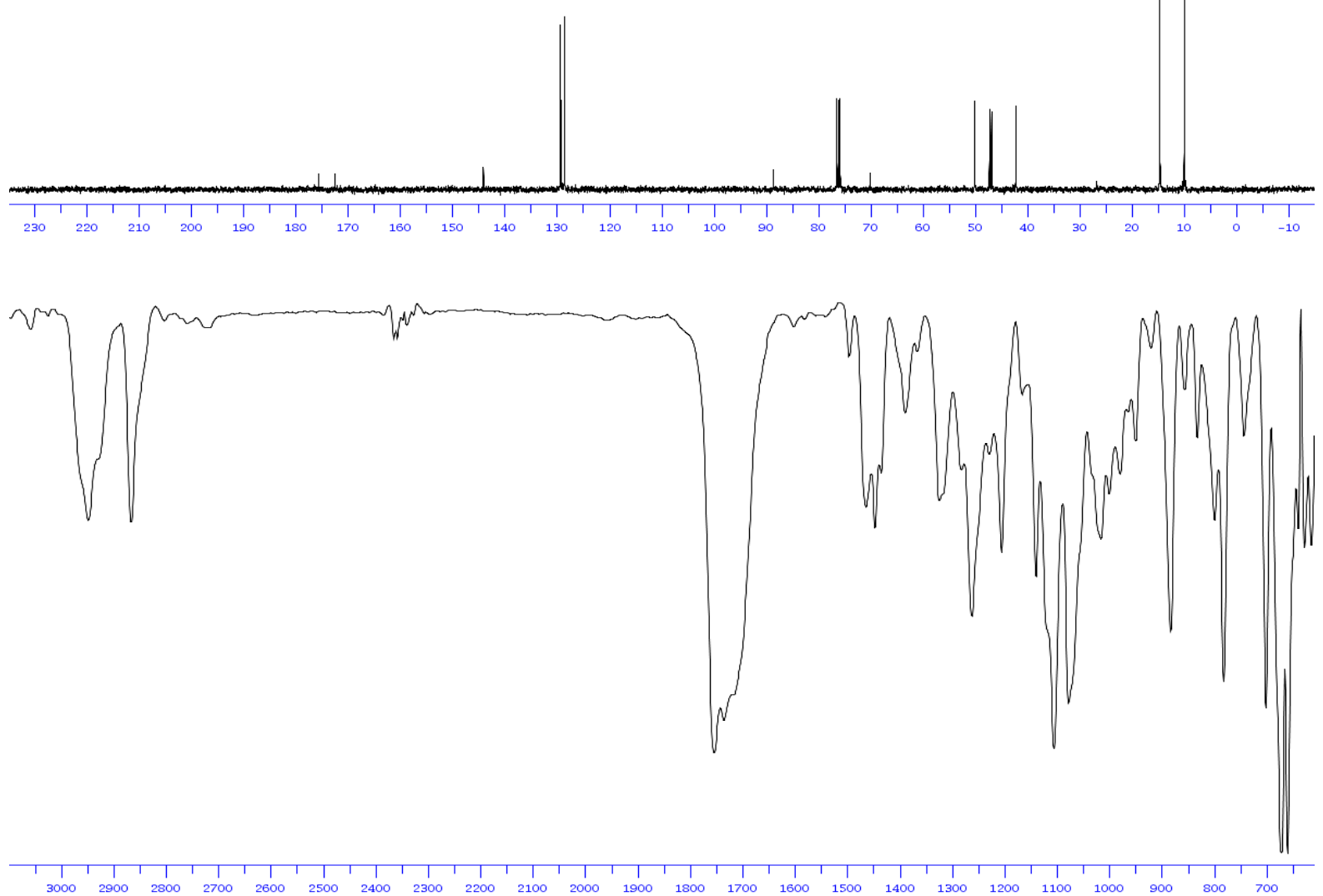


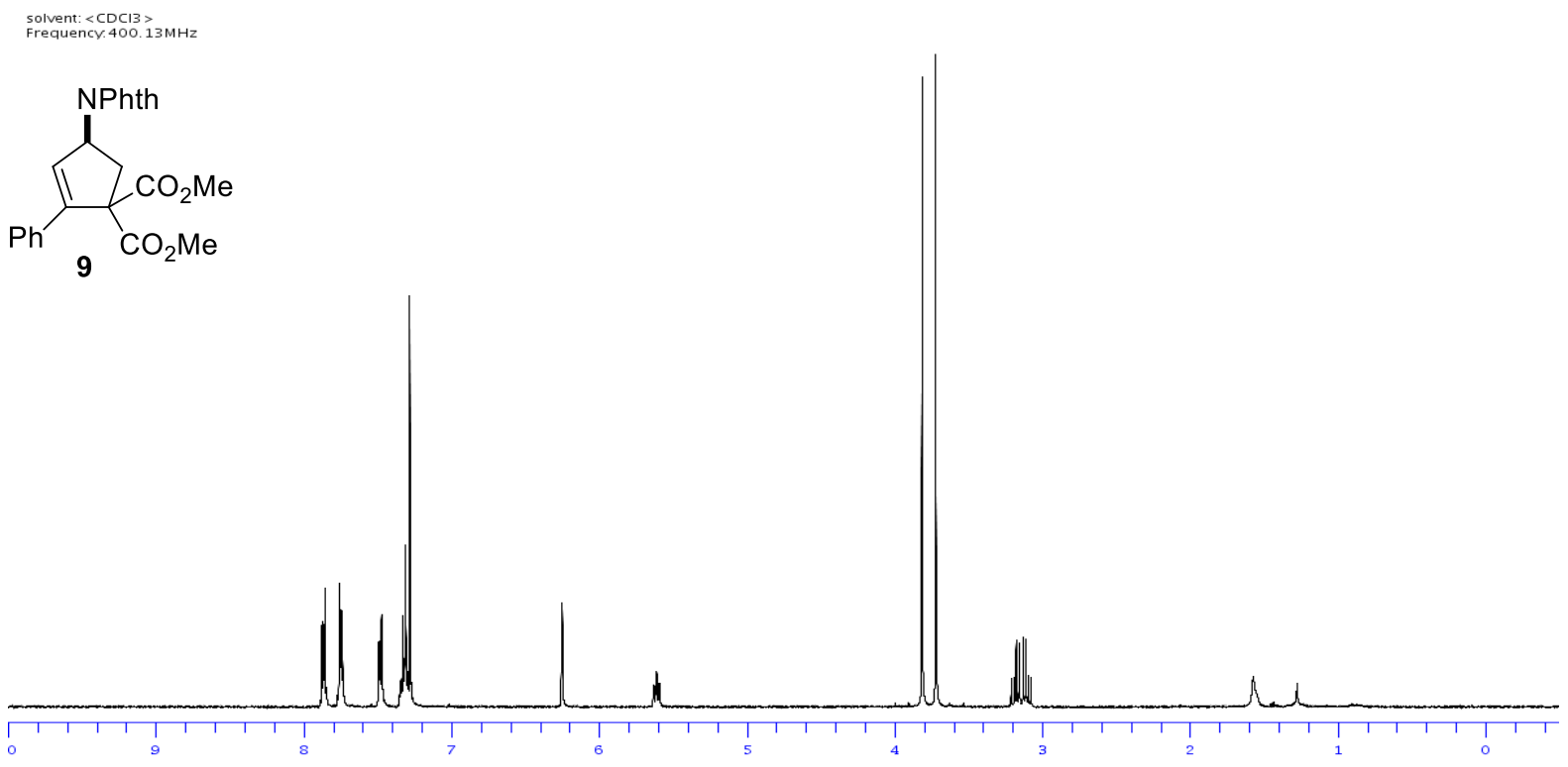

solvent: $<\mathrm{CDCl} 3>$
Frequency. $100.612769 \mathrm{MHz}$

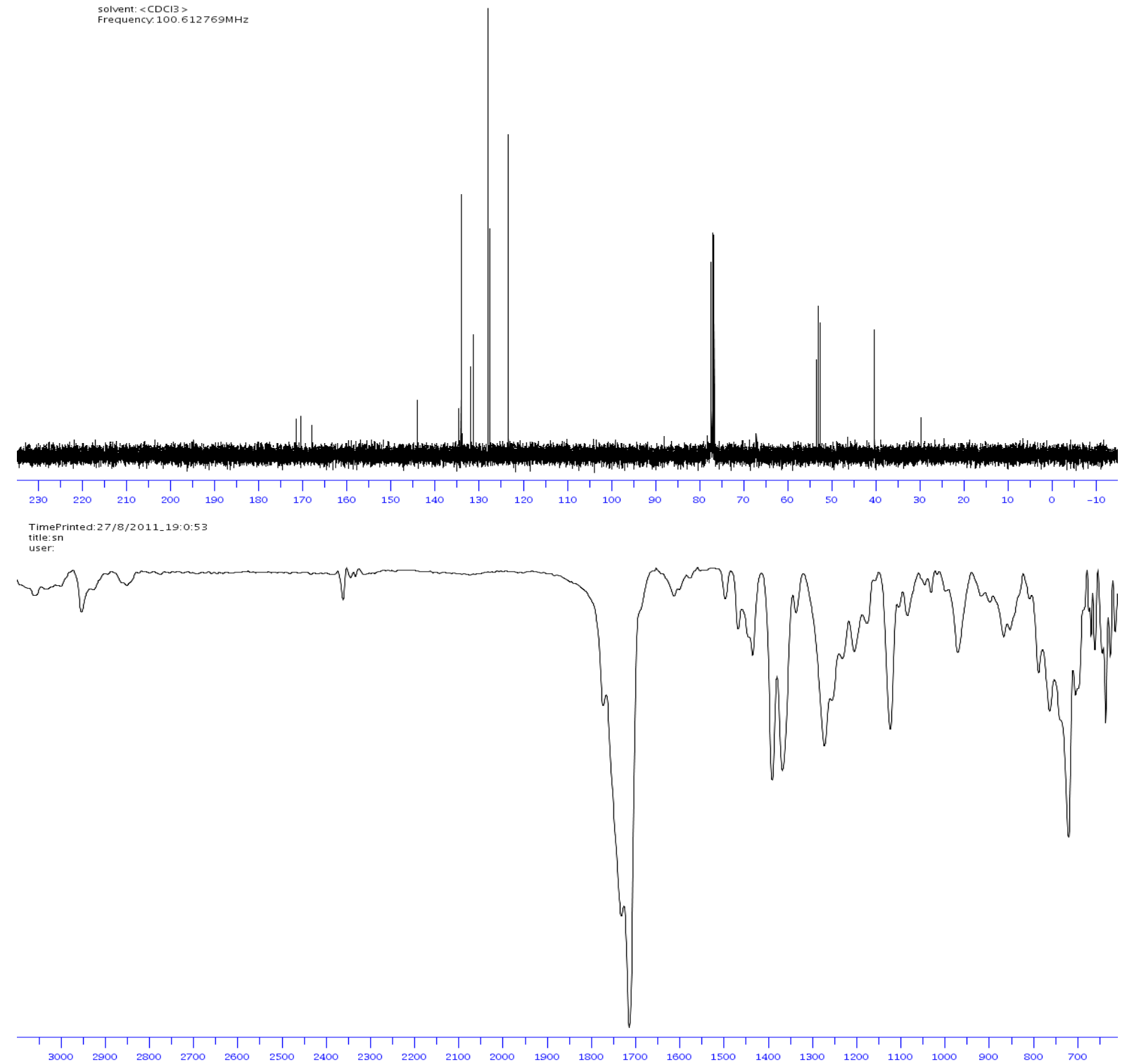




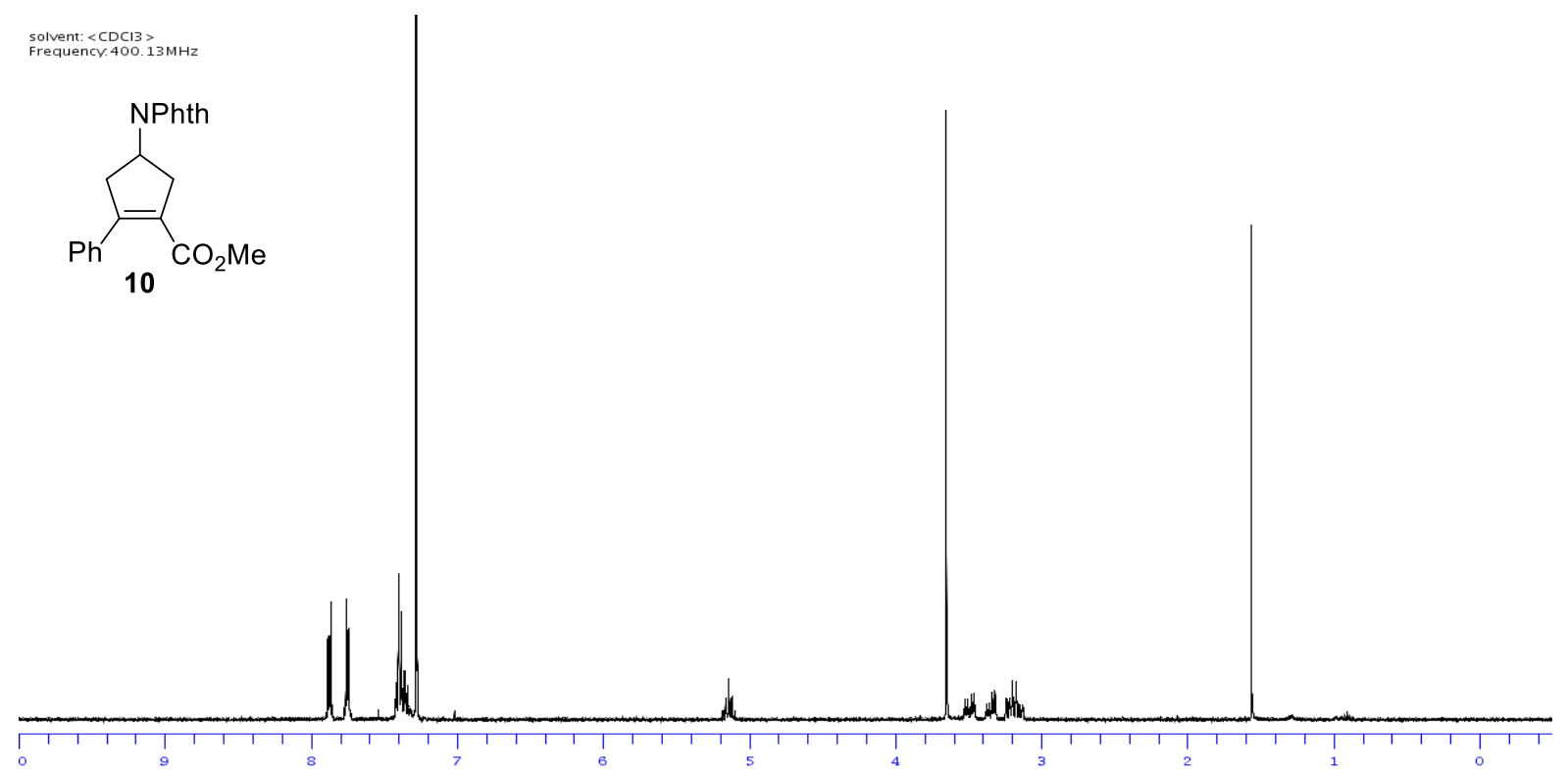

Solvent $<$ CDC13 $>$
Frequency.100.612769MHz

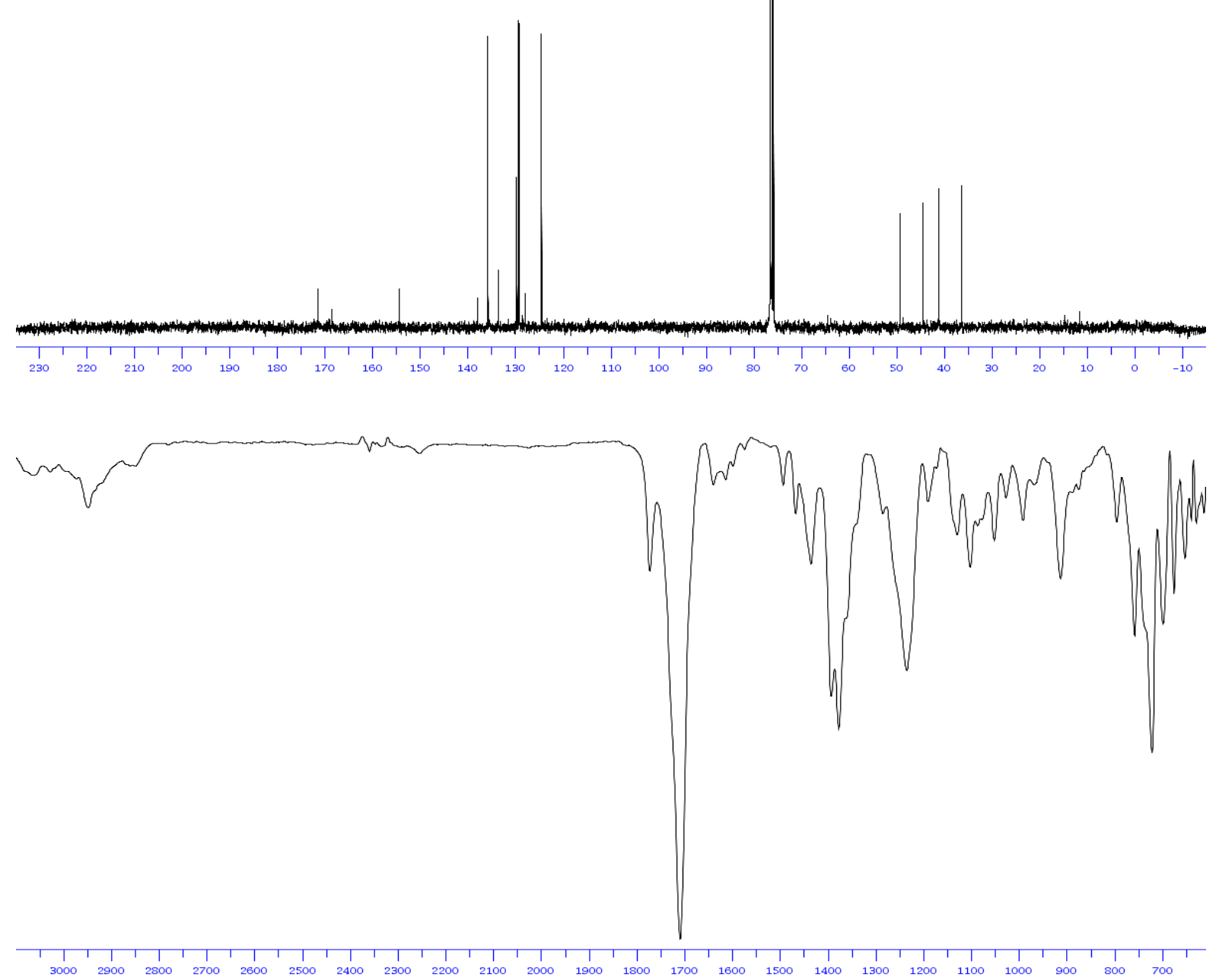



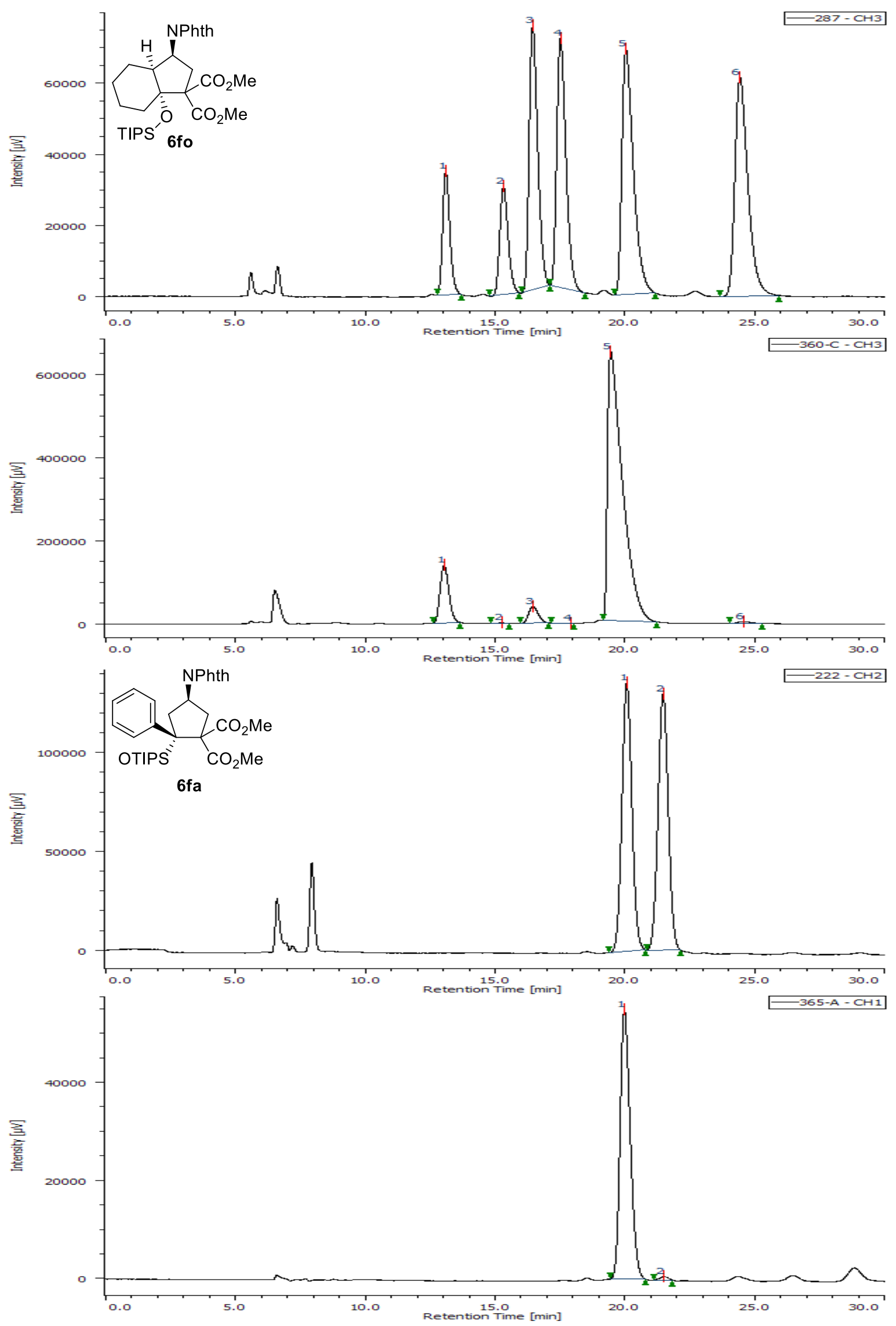

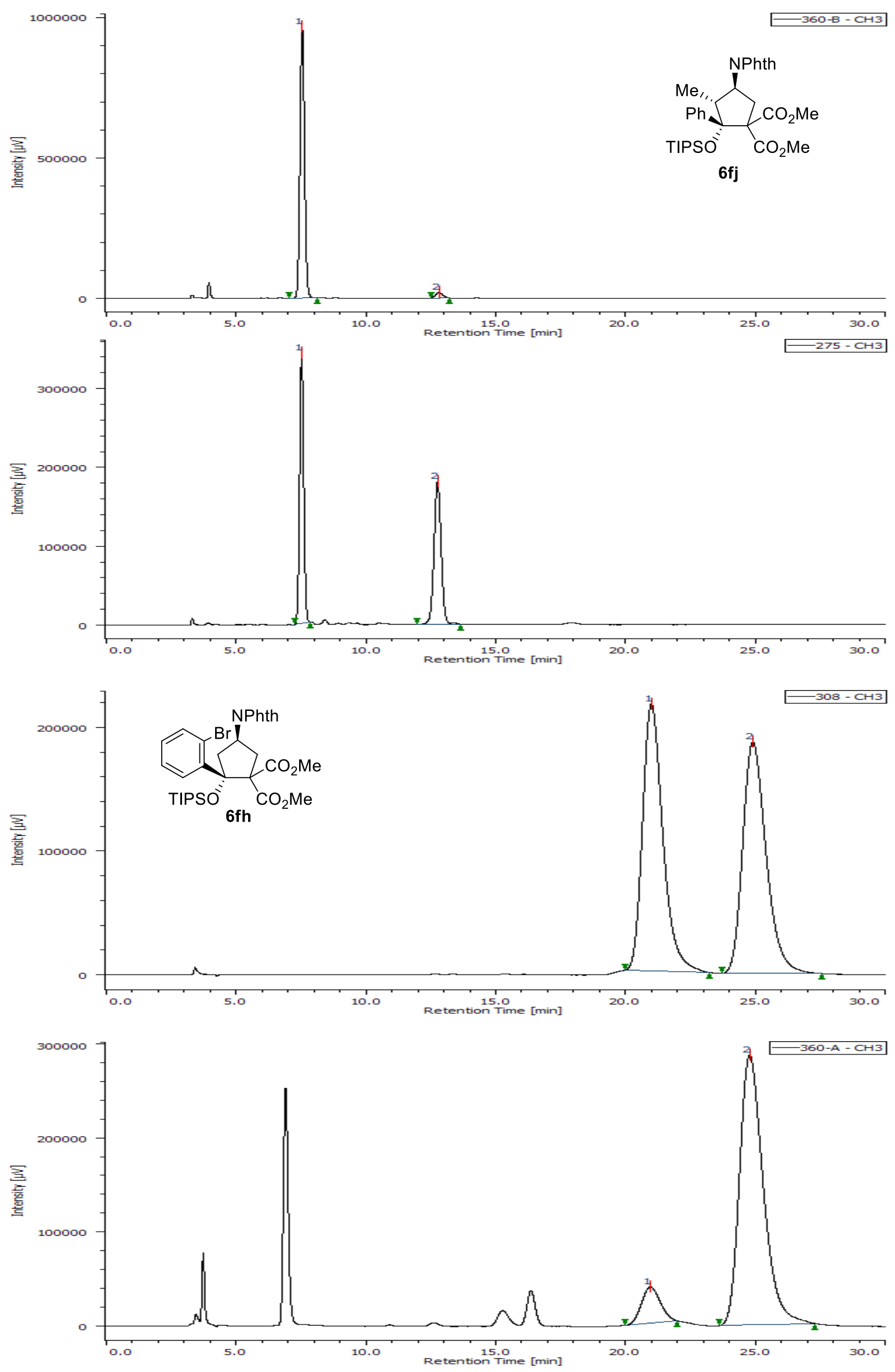

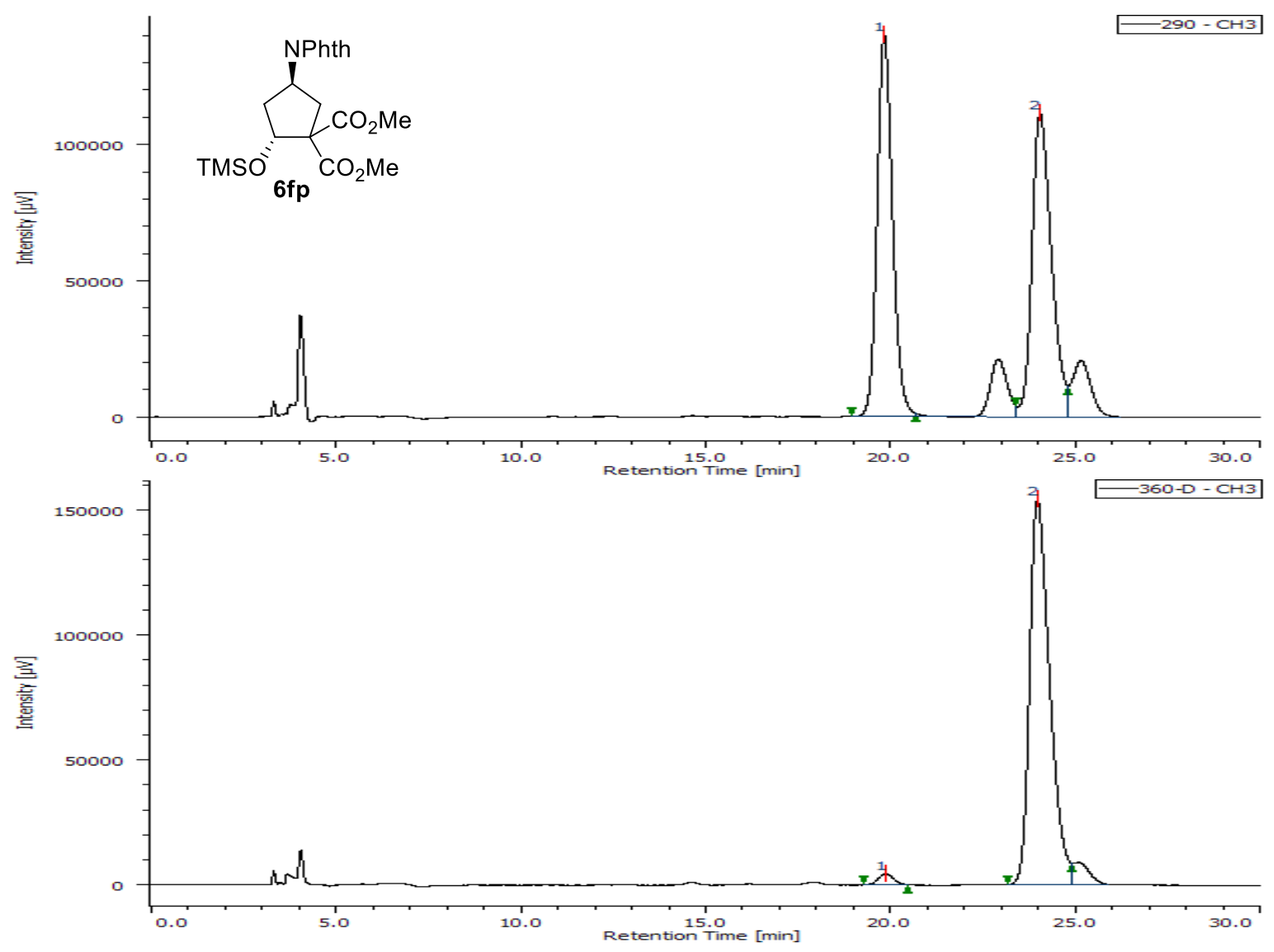

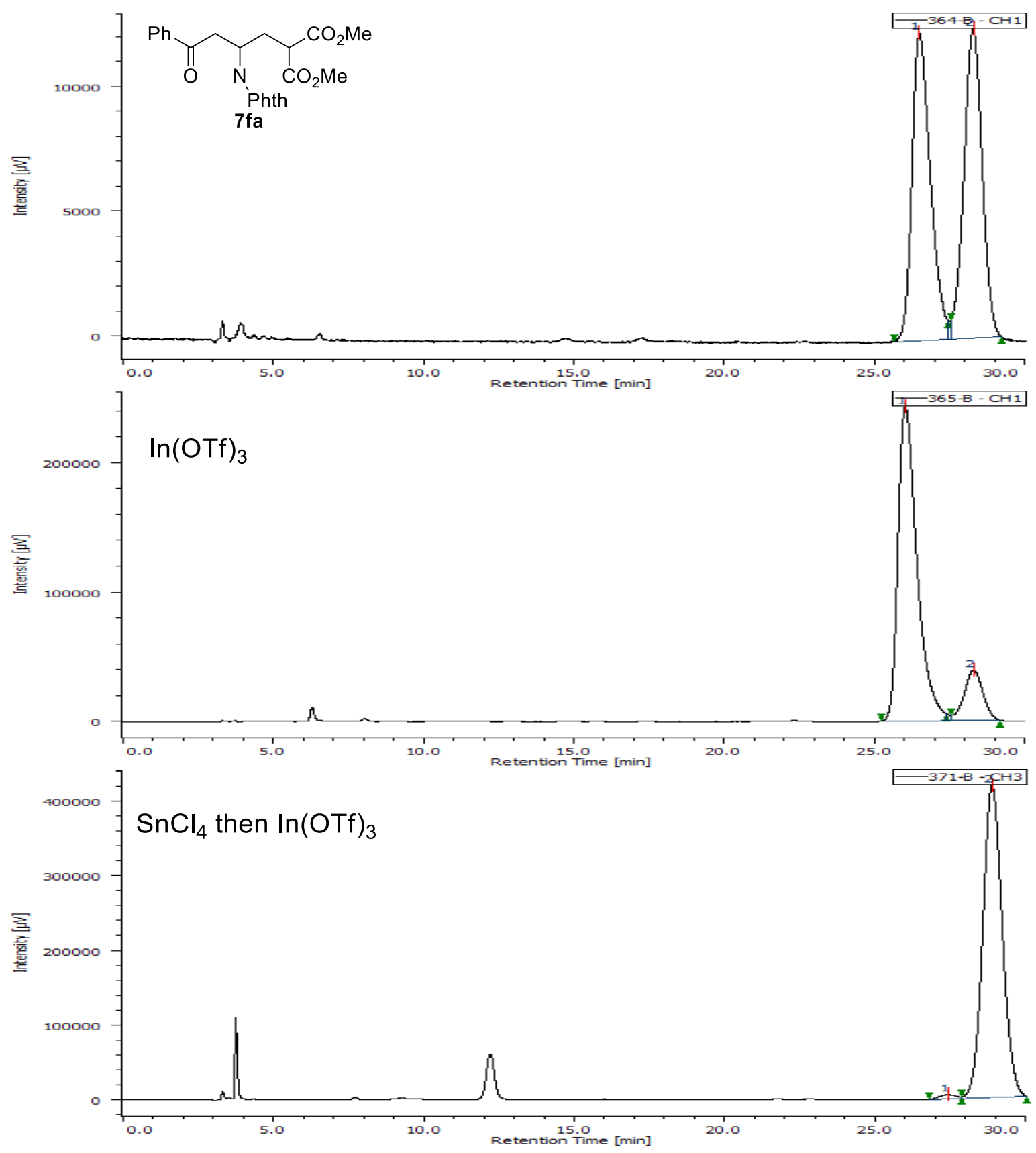

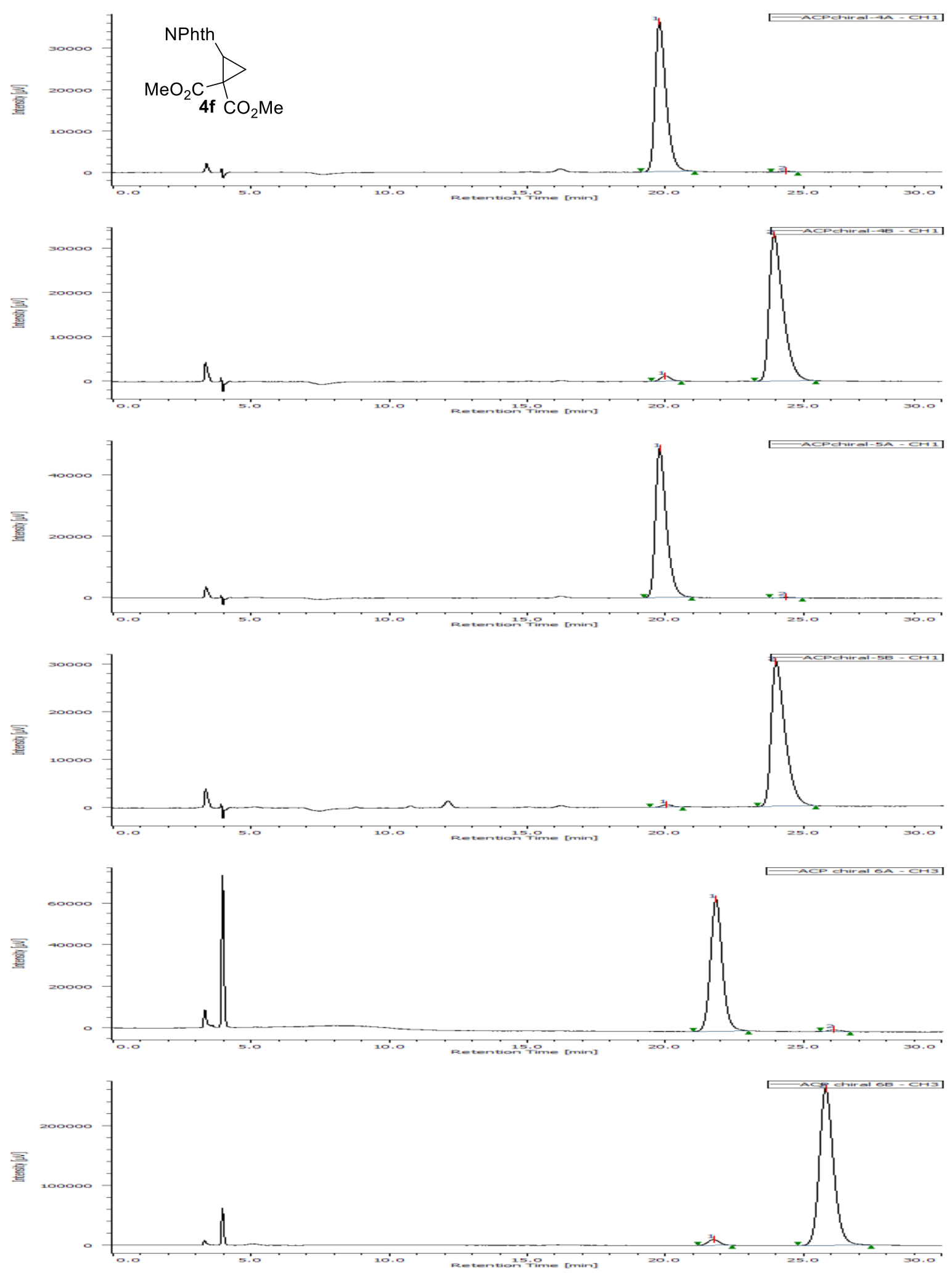